\title{
Tribo-Mechanical Investigation of the Functional Components used in Flexible Energy Harvesting Devices
}

Nicholas J. Morris

Follow this and additional works at: https://researchrepository.wvu.edu/etd

\section{Recommended Citation}

Morris, Nicholas J., "Tribo-Mechanical Investigation of the Functional Components used in Flexible Energy Harvesting Devices" (2015). Graduate Theses, Dissertations, and Problem Reports. 6265.

https://researchrepository.wvu.edu/etd/6265

This Dissertation is protected by copyright and/or related rights. It has been brought to you by the The Research Repository @ WVU with permission from the rights-holder(s). You are free to use this Dissertation in any way that is permitted by the copyright and related rights legislation that applies to your use. For other uses you must obtain permission from the rights-holder(s) directly, unless additional rights are indicated by a Creative Commons license in the record and/ or on the work itself. This Dissertation has been accepted for inclusion in WVU Graduate Theses, Dissertations, and Problem Reports collection by an authorized administrator of The Research Repository @ WVU.

For more information, please contact researchrepository@mail.wvu.edu. 


\title{
Tribo-Mechanical Investigation of the Functional Components used in Flexible Energy Harvesting Devices
}

\author{
Nicholas J. Morris, B.S. \\ Dissertation submitted to \\ the \\ Benjamin M. Statler College of Engineering and Mineral Resources \\ at West Virginia University \\ in partial fulfillment of requirements \\ for the degree of \\ Doctor of Philosophy \\ in \\ Mechanical Engineering
}

\author{
Konstantinos Sierros, Ph.D, Chair \\ Marvin Cheng, Ph.D \\ Xingbo Liu, Ph.D \\ Edward Sabolsky, Ph.D \\ George Kiriakidis, Ph.D
}

\section{Department of Mechanical and Aerospace Engineering \\ Morgantown, West Virginia \\ 2015}

Keywords: flexible devices, piezoelectric energy harvesting, transparent conductive oxides, tribology

Copyright 2015 


\section{ABSTRACT \\ Tribo-Mechanical Investigation of the Functional Components used in Flexible Energy Harvesting Devices}

\section{Nicholas J. Morris}

During the previous decade, the development of energy harvesting devices based on piezoelectric materials has garnered great interest. The ability to capture ambient mechanical energy and convert it to useable electricity is a potential solution to the ever-growing energy crisis. One of the most attractive functional materials used in these devices is zinc oxide ( $\mathrm{ZnO})$. This material's relative low cost and ease of large-area processing has spurred numerous device designs based around it. The ability to grow $\mathrm{ZnO}$ nanostructures of various geometries with lowtemperature chemical methods makes this material even more attractive for flexible devices. Although numerous device architectures have been developed, the long-term mechanical reliability has not been addressed.

This work focuses on the fabrication and mechanical failure analysis of the flexible components typically used in piezoelectric energy harvesting devices. A three-phase iterative design process was used to fabricate prototypical piezoelectric nanogenerators, based on $\mathrm{ZnO}$ nanowires. An output of several millivolts was achieved under normal contact and microtensile loading, but device failure occurred after only a few loading cycles, in all cases. Ex situ failure analysis confirmed the primary sources of failure, which became the focus of further, component-level studies. Failure was primarily seen in the flexible electrodes of the nanogenerating devices, but was also observed in the functional piezoelectric layer itself.

Flexible electrodes comprised of polyester substrates with transparent conductive oxide (TCO) coatings were extensively investigated under various loading scenarios to mimic tribomechanical stresses applied during fabrication and use in flexible contact-based devices. The durability of these films was explored using microtensile testing, spherical nanoindentation, controlled mechanical buckling, stress corrosion cracking, and shear-contact reciprocating wear. The electro-mechanical performance and reliability of functional $\mathrm{ZnO}$ films and nanostructures were also studied. $\mathrm{ZnO}$ was deposited on rigid and flexible substrates for investigations including controlled buckling, and contact-based rolling/sliding scenarios. Numerous in situ and ex situ analytical techniques were used to characterize component-level failure mechanisms, including two-probe electrical resistance, optical microscopy, SEM, AFM, and stylus profilometry.

Experimental results show that there is a strong relation between crack onset strain values, during microtensile and controlled bucking loading, and coating thickness. Relatively high crack onset values were observed for both thinner coatings and those patterned using photolithography and wet chemical etching techniques. Tribological experiments show that although piezoelectric $\mathrm{ZnO}$ films produce a measurable electrical output during combined rolling/sliding contact, cohesive wear of the oxide and adhesive wear between oxide and substrate is present and detrimental to sustained film functionality. 


\section{ACKNOWLEDGEMENTS}

I would like to sincerely thank my research advisor, Dr. Kostas Sierros, for his unwavering guidance and support throughout my undergraduate and graduate career. Without him, this work would have not been possible. I would also like to thank my committee members, Dr. Marvin Cheng, Dr. Xingbo Liu, Dr. Ed Sabolsky, and Dr. George Kiriakidis for their continued support during this project.

I would also like to thank several fellow lab members including Sean Cronin, Teddy Bejitual, and Derrick Banerjee.

I am also grateful for assistance and training from Kolin Brown, Harley Hart, Marcela Redigolo, and Weiquaing Ding of the WVU Shared Research Facilities.

Finally, this work is dedicated to my parents Gary and Lynn Morris and my wife Rachel. Your love and support made this possible.

This work was financially supported by the National Science Foundation (NSF) through the Graduate Research Fellowship Program. 


\section{TABLE OF CONTENTS}

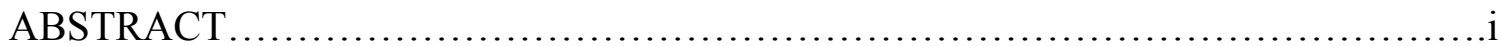

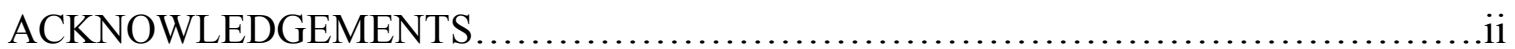

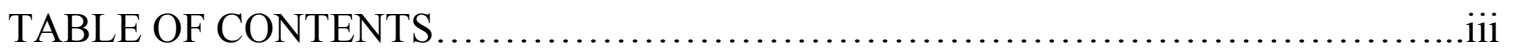

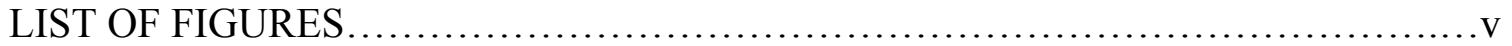

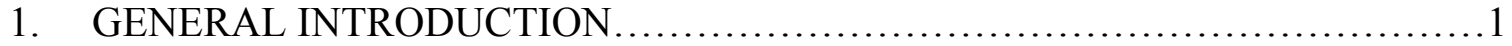

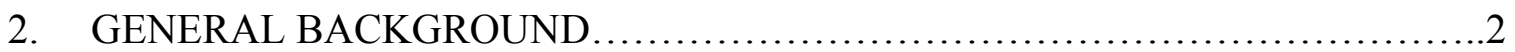

2.1 Flexible Electronic Devices..............................................

2.2 Flexible Substrates..................................................... 4

2.3 Transparent Conductive Oxides...................................... 5

2.4 Piezoelectric Zinc Oxide..................................................6

2.5 Mechanical Properties of Thin Film Electrodes.............................12

2.6 Tribo-Mechanical Properties of Zinc Oxide..................................14

2.7 Mechanical Properties of Zinc Oxide Nanowires..............................16

2.8 Experimental Methods.................................................. 18

3. FABRICATION AND CHARACTERIZATION OF FLEXIBLE PIEZOELECTRIC

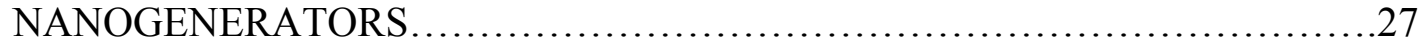

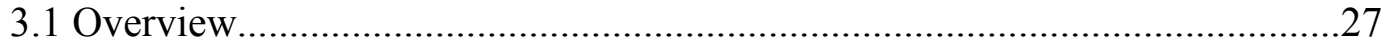

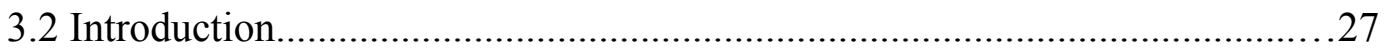

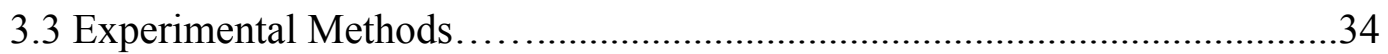

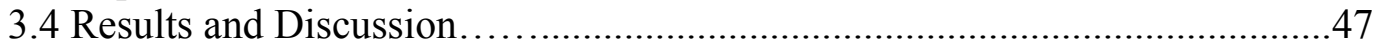

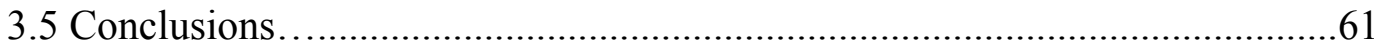

4. MONOTONIC MECHANICAL LOADING STUDIES OF INDIUM TIN OXIDE (ITO) ELECTRODES ON POLYESTER SUBSTRATES...............................63

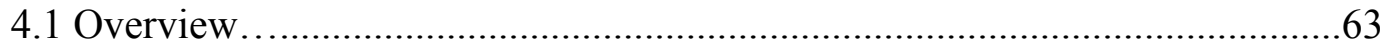

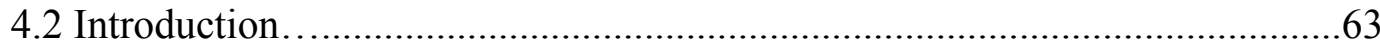

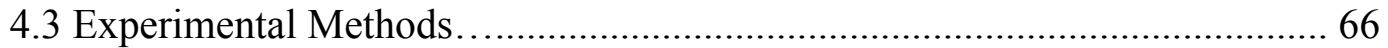

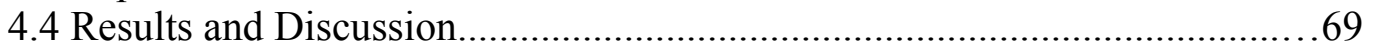

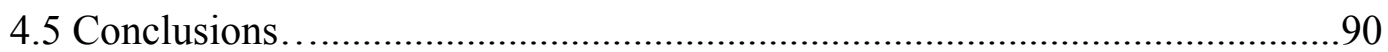

5. CYCLIC TRIBO-CHEMCIAL STUDIES OF INDIUM TIN OXIDE (ITO) FILMS ON POLYESTER

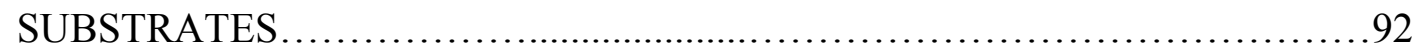

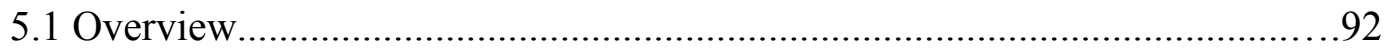

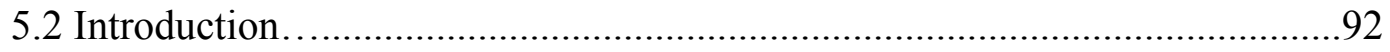

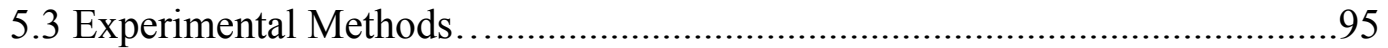

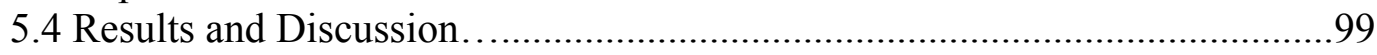

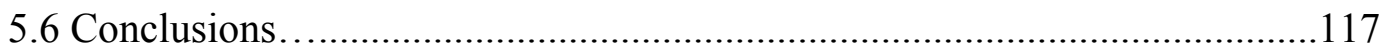

6. TRIBO-MECHANICAL STUDIES OF ZINC OXIDE (ZNO) FOR USE IN FLEXIBLE CONTACT-BASED PIEZOELECTRIC DEVICES...... 


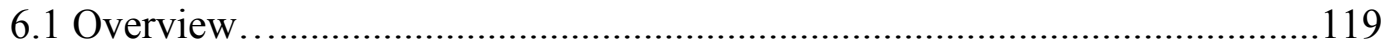

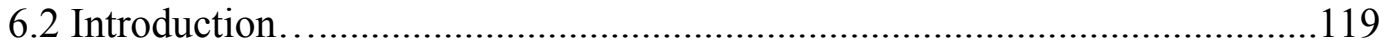

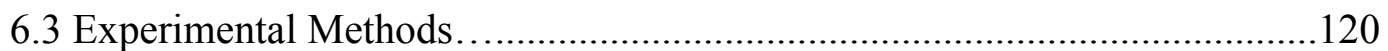

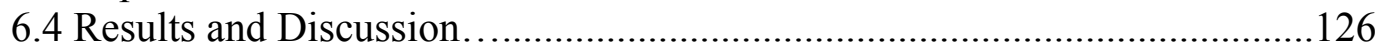

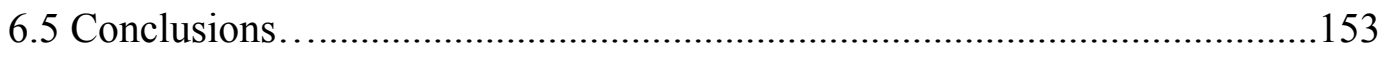

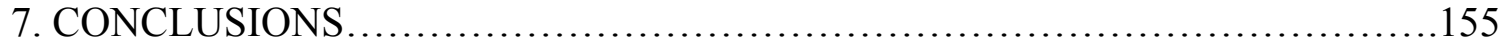

8. REFERENCES..................................................... 158 


\section{LIST OF FIGURES}

Fig. 2.1 a) Wurtzite structure of $\mathrm{ZnO}$ b) Generation of a piezoelectrical potential in a $\mathrm{ZnO}$ crystal.[53] ......................................................

Fig. 2.2 Side-view of custom-built controlled buckling experimental apparatus........19

Fig. 2.3 Experimental schematic of stress-corrosion cracking apparatus.................21

Fig. 2.4 Schematic of custom-built reciprocating wear apparatus....................22

Fig. 2.5 Schematic of custom-built reciprocating rolling-contact experimental apparatus with in situ electrical monitoring..........................................23

Fig. 2.6 Schematic of reciprocating wear apparatus and ball bearing holder used in this study................................................................. 25

Fig. 3.1 a) SEM micrograph of vertically-aligned $\mathrm{ZnO}$ nanowires b) TEM images of $\mathrm{ZnO}$ nanowires c) Experimental setup for measuring piezoelectric output of individual $\mathrm{ZnO}$ nanowires using a conductive AFM tip [117] ...................38

Fig. 3.2 a) Schematic of vertically-aligned nanogenerator device with zigzag silicon top electrode b-d) SEM images of various device components. [85]..............22

Fig. 3.3 Current output of the zigzag substrate device under ultrasonic wave loading

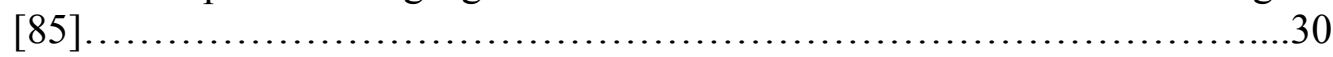

Fig. 3.4 Design and fabrication procedure of multilayered nanogenerator with SEM images of $\mathrm{ZnO}$ nanowires and gold-coated conical counterface [46]...........30

Fig. 3.5 a) Single-wire nanogenerator device and piezoelectric potential modeling b-d) Current and voltage output of single-wire nanogenerator device under oscillating fingertip bending. [44].

Fig. 3.6 Fabrication of vertically-aligned nanowire generator based on a polystyrene

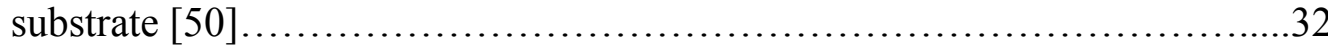

Fig. 3.7 Schematic of nanogenerator design progression for Phases I-III..................37

Fig. 3.8 SEM micrographs of hydrothermally grown nanowires with a growth solution concentration of $0.025 \mathrm{M}$ and a growth temperature of $70^{\circ} \mathrm{C}$ with a $150^{\circ} \mathrm{C}$ seed layer annealing and a growth time of a-b) 24 hours c) 48 hours.................39

Fig. 3.9 SEM micrographs of the tip geometry under a) $0.01 \mathrm{M}$ concentration, $95^{\circ} \mathrm{C}$, and 24 hours of growth time, b) $0.025 \mathrm{M}$ concentration, $70^{\circ} \mathrm{C}$, and 48 hours of growth time.

Fig. 3.10 X-ray diffraction of grown $\mathrm{ZnO}$ nanowires on a) $\mathrm{ZnO}$ seed layer on glass b) on gold-coated glass c) on gold coated PEN.

Fig. 3.11 SEM image of hydrothermally grown nanowires on a gold-coated PEN substrate. .42

Fig. 3.12 SEM micrograph of patterned $\mathrm{ZnO}$ nanowire arrays using photolithography techniques............................................................ 44

Fig. 3.13 Fabrication process of the Phase II, flexible PEN-based sensor design with image of a fabricated device under fingertip loading........................47

Fig. 3.14 Diagram of repeated micro-indentation testing of Phase II devices...........48

Fig. 3.15 a) Fingertip buckling loading of flexible Phase II device design b) Open-circuit voltage measured under buckling .......................................50

Fig. 3.16 Electrical voltage output of PEN-based device under various applied contact pressures using the microindentation testing scheme..........................51 
Fig. 3.17 Superposition of peak electrical voltage output curves under each of the applied contact pressures...........................................................................52

Fig. 3.18 SEM failure analysis of $\mathrm{ZnO}$ nanowires after repeated loading at $392 \mathrm{kPa}$....53

Fig. 3.19 SEM failure analysis of top gold electrode after repeated loading at $392 \mathrm{kPa}$ of contact pressure....................................................53

Fig. 3.20 Load-displacement curves of 10 micron spherical tip nanoindentation on varied densities of $\mathrm{ZnO}$ nanowires and calculated hardness values..................55

Fig. 3.21 Schematic of fabrication process for the PDMS-based device design..........59

Fig. 3.22 a) Fingertip tensile loading of PDMS-based device with b) measured electrical

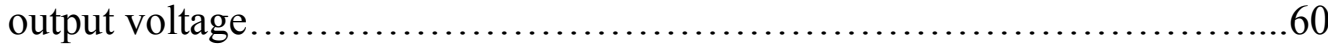

Fig. 3.23 Regions of expected failure mechanisms for the Phase III device structure.....61

Fig. 4.1 Schematic of ITO patterns fabricated using photolithography and wet etching techniques, showing (a) characteristic lengths and (b) angles. Dimensions are in microns and degrees, respectively.

Fig. 4.2 Electromechanical behavior of ITO-coated PET with sheet resistances of 100

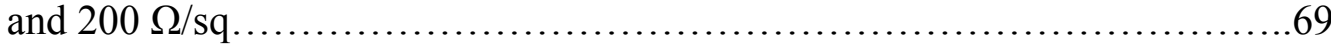

Fig. 4.3 ITO cracking and delamination of an ITO-coated PET sample tensile tested above $6 \%$ strain. Arrows indicate force direction............................70

Fig. 4.4 Load displacement spherical indentation curves for ITO coatings sputtered on polyester substrates............................................... 71

Fig. 4.5 Failure pattern evolution for a $100 \mathrm{~nm}$ thick ITO coating as normal spherical loading increases. The scale bar is equal to $5 \mu \mathrm{m}$ for all images..............72

Fig. 4.6 Scanning electron microscope image of a part of a spherical indent on a 200 $\mathrm{nm}$ thick ITO coated PET, under a $200 \mathrm{mN}$ load..........................73

Fig. 4.7 Ring crack patterns on ITO surfaces for different normal loads and film thicknesses.

Fig. 4.8 Indent imprint radius versus applied load for coatings with different thicknesses. Inset optical image shows and indent of $50 \mathrm{~nm}$ thick coating subjected to a $150 \mathrm{mN}$ load (scale bar is equal to $5 \mu \mathrm{m}$ )...................75

Fig. 4.9 Semi-log plot of average contact pressure versus applied load for ITO coated PET. The thickness of the ITO coating is $200 \mathrm{~nm}$.......................77

Fig. 4.10 Ring crack spacing versus applied normal load for 50, 100, and $200 \mathrm{~nm}$ thick ITO coatings sputtered on PET substrates. Error bars represent stand deviation of the data...

Fig. 4.11 a) Typical panoramic view of a scratched ITO surface. Coating thickness is equal to $50 \mathrm{~nm}$ and the total scratch length is equal to $0.5 \mathrm{~mm}$, b) optical image showing the initiation of through thickness cracking, c) optical image showing the initiation of coating spallation. Both optical images have scale bars equal to

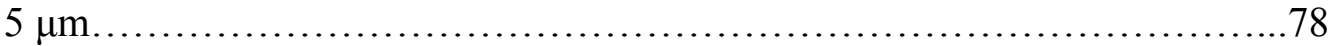

Fig. 4.12 a) Typical tapping-mode AFM image showing coating cracking: vertical range $=348 \mathrm{~nm}$ b) tapping-mode AFM image showing coating buckling spallation; vertical range $=830 \mathrm{~nm}$. The coating has a thickness equal to $250 \mathrm{~nm}$. Arrows indicate the direction of scratching. Both micrographs have scale bars equal to

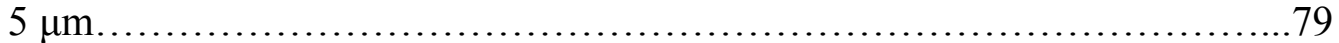

Fig. 4.13 SEM image of a $200 \mathrm{~nm}$ thick ITO coating showing spalled areas and interfacial failure during scratch testing. .79 
Fig. 4.14 Graph showing the relation of applied critical load and coating thickness for cracking and spallation failure modes during scratch testing.................80

Fig. 4.15 Normalized electrical resistance versus applied strain for smaller size patterned ITO-coated PET films under a) tension and b) compression.

Fig. 4.16 Normalized electrical resistance versus applied strain for larger size patterned ITO-coated PET films under a) tension and b) compression..................81

Fig. 4.17 Crack onset strain from change in electrical resistance monitoring of ITOcoated PET films.

Fig. 4.18 Optical microscopy images of circular patterned ITO-coated PET films under tensile buckling deformation mode showing cracking initiation and propagation up to $6 \%$ strain. Arrows indicate the buckling direction...........84

Fig. 4.19 Optical microscopy images of circular patterned ITO coated PET films under compressive buckling deformation showing cracking initiation and propagation up to $6 \%$ strain. Arrows indicate the buckling direction.....................84

Fig. 4.20 Optical microscopy images of square patterned ITO-coated PET films under tensile buckling deformation mode showing cracking initiation and propagation up to $6 \%$ strain. Arrows indicate the buckling direction

Fig. 4.21 Optical microscopy images of square patterned ITO-coated PET films under compressive buckling deformation mode showing cracking initiation and propagation up to $6 \%$ strain. Arrows indicate the buckling direction...........86

Fig. 4.22 Optical microscopy images of zigzag patterned ITO-coated PET films under tensile buckling deformation mode showing cracking initiation and propagation up to $6 \%$ strain. Arrows indicate the buckling direction.

Fig. 4.23 Optical microscopy images of zigzag patterned ITO-coated PET films under compressive buckling deformation mode showing cracking initiation and propagation up to $6 \%$ strain. Arrows indicate the buckling direction...........87

Fig. 4.24 SEM micrographs of patterned ITO-coated PET films a) under tensile and b) under compressive buckling deformation. Arrows indicate the buckling direction......................................................... 88

Fig. 4.25 Crack onset strain from in situ optical microscopy monitoring of ITO-coated PET films

Fig. 4.26 Normalized electrical resistance at COS measured using electrical resistance data for ITO-coated PEN films

Fig. 5.1 Electrical resistance versus time for a $200 \Omega /$ sq, ITO-coated PET sample immersed in acrylic acid of $0.1 \mathrm{M}$ concentration

Fig. 5.2 a) TOF-SIMS depth profile of an un-aged test coupon of PSA on ITO and b) TOF-SIMS depth profile of an aged coupon of PSA on ITO. The sample was

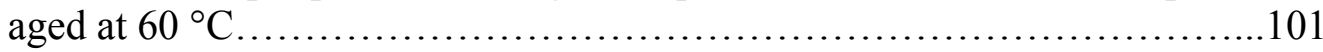

Fig. 5.3 Open-circuit potential versus time curve for an ITO film in $0.1 \mathrm{M}$ acrylic acid.

Fig. 5.4 Electrochemical impedance specra for ITO in $0.1 \mathrm{M}$ acrylic acid: a) Nyquist plot and b) Bode (frequency and phase angle) plots.

Fig. 5.5 Normalized electrical resistance versus experimental time for unstrained ITOcoated PET immersed in acrylic acid of various concentrations.............104

Fig. 5.6 SEM image showing stress-corrosion cracking formation in an ITO-coated PET, $100 \Omega /$ sq, immersed in $0.3 \mathrm{M}$ acrylic acid when an external bending strain of $0.96 \%$ is applied......................................... 105 
Fig 5.7 a) Electromechanical behavior of ITO-coated PET samples immersed in $0.1 \mathrm{M}$ acrylic acid for various applied bending strains b) SEM image showing a region of an ITO-coated PET strained at $0.78 \%$ in $0.1 \mathrm{M}$ acrylic acid...........106

Fig. 5.8 Normalized electrical resistance versus number of reciprocating cycles (semi$\log$ plot) in dry conditions.......................................... 108

Fig. 5.9 Normalized electrical resistance versus number of reciprocating cycles (semi$\log$ plot) in $0.1 \mathrm{M}$ acrylic acid..................................... 108

Fig. 5.10 Percentage increase in electrical resistance, after dry and we sliding, of the topreciprocating ITO surface against number of sliding cycles (semi-log plot)..110

Fig. 5.11 Weight loss versus number of reciprocating cycles (log-log plot) for both dry and wet sliding of the top ITO surface.............................. 112

Fig. 5.12 Weight loss versus number of reciprocating cycles (log-log plot) for both dry and wet sliding of the bottom ITO surface.

Fig. 5.13 Typical surface profiles of bottom worn ITO counterface for both dry (a) and wet (b) conditions, after 12,240 reciprocating cycles..................... 114

Fig 5.14 (Left) optical image showing a top ITO counterface for 12,240 cycles under pure dry sliding conditions. (Right) optical image showing a top ITO surface sliding for 12,240 cycles in the presence of $0.1 \mathrm{M}$ acrylic acid. (Arrows

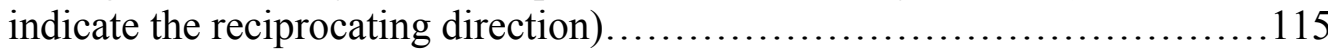

Fig. 5.15 (Left) Optical image showing a bottom ITO counterface for 12,240 cycles under pure dry sliding conditions. (Right) optical image showing a bottom ITO counterface for 12,240 cycles in the presence of $0.1 \mathrm{M}$ acrylic acid. (Arrows indicate the reciprocating direction)

Fig. 5.16 (Left) SEM image showing a bottom ITO counterface for 1020 cycles in the presence of $0.1 \mathrm{M}$ acrylic acid. (Right) SEM image showing a bottom ITO surface for 1020 cycles under dry conditions...........................117

Fig. 6.1 Contact angle values of PET and PEN substrate surfaces. Error bars indicate standard deviation of data. Inset image shows a typical contact angle measurement on a PEN surface.

Fig. 6.2 AFM images of $\mathrm{ZnO}$ films sputtered on PET. a) $\mathrm{ZnO}$ thickness is $65 \mathrm{~nm}$ and Zrange is $28 \mathrm{~nm}$. b) $\mathrm{ZnO}$ thickness is $325 \mathrm{~nm}$ and Z-range is $76 \mathrm{~nm}$. c) $\mathrm{ZnO}$ thickness is $680 \mathrm{~nm}$ and Z-range is $74 \mathrm{~nm}$. The scale bar is equal to $650 \mathrm{~nm}$ in all cases.

Fig. 6.3 Contact angle values for $\mathrm{ZnO}$ versus different $\mathrm{UV}$ exposure times, a) water droplet on a non-UV exposed $\mathrm{ZnO}$ surface, b) water droplet on a 60 minute UV exposed $\mathrm{ZnO}$ surface.

Fig. 6.4 a) AFM topographical and b) friction map images of UV exposed $\mathrm{ZnO}$ surface

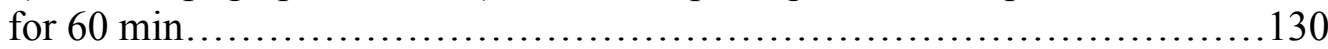

Fig. 6.5 Friction signal versus applied normal load for $\mathrm{ZnO}$ single asperity sliding against a $\mathrm{ZnO}$ film surface before and after exposure to UV light for 60 minutes.

Fig. 6.6 Optical transmission versus wavelength for various thicknesses of $\mathrm{ZnO}$-coated PET ..............................................................132

Fig. 6.7 X-ray diffraction pattern of a $2 \theta$ scan for a sol-gel deposited $\mathrm{ZnO}$ film using a zinc acetate dehydrate solution. 
Fig. 6.8 Load versus penetration depth indentation curves for $1 \mu \mathrm{m}$ thick $\mathrm{ZnO}$ films. A

Berkovich diamond tip was used....................................134

Fig. 6.9 Optical images showing a panoramic view of the scratch which has a total length equal to $0.5 \mathrm{~mm}$ (top) and the major scratch failure mechanisms (bottom) for a $75 \mathrm{~nm} \mathrm{ZnO}$ coated PET sample........................135

Fig. 6.10 Critical normal loads for cracking and delamination as a function of film thickness during scratch testing ....................................... 136

Fig. 6.11 SEM image showing secondary failure mechanisms for a $650 \mathrm{~nm} \mathrm{ZnO} \mathrm{film}$ deposited on a PET substrate.

Fig. 6.12 Typical optical micrographs showing crack initiation and propagation for a $\mathrm{ZnO}$ film, $75 \mathrm{~nm}$ thick, sputtered on a PET substrate under controlled compression conditions up to 3\% strain. Arrows indicate the compression

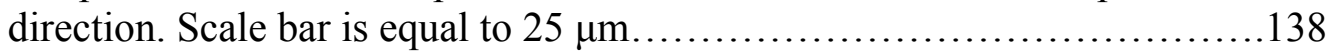

Fig. 6.13 SEM image of crack morphology for a $650 \mathrm{~nm}$ thick $\mathrm{ZnO}$ film sputtered on PET. Arrows indicate compression direction............................139

Fig. 6.14 Graph showing in situ optically measured crack-onset strain versus film thickness for ZnO films sputtered on PET and PEN substrates.

Fig. 6.15 Weight loss of $\mathrm{ZnO}$ films versus number of reciprocating rolling-sliding cycles. The counterface is a stainless steel cylinder....................... 141

Fig. 6.16 Weight gain of the rolling stainless-steel counterface versus number of reciprocating cycles................................................ 142

Fig. 6.17 Optical images of non-UV exposed $\mathrm{ZnO}$ surfaces taken after various amounts of reciprocating rolling. The rolling direction is the same for all images......143

Fig. 6.18 Optical image of 60 minute UV exposed $\mathrm{ZnO}$ surfaces taken after various amounts of reciprocating rolling cycles. The rolling direction is the same for all images............................................................. 144

Fig. 6.19 Optical images of stainless steel cylinder surfaces. A) Initial counterface, b) surface after 25,000 rolling cycles on a non-UV exposed $\mathrm{ZnO}$ surface, c) cylinder surface after 25,000 reciprocating cycles on a 60 minute UV exposed $\mathrm{ZnO}$ film surface................................................145

Fig. 6.20 Voltage response of preliminary device design subjected to rolling for a total of 150 cycles at a frequency of $0.5 \mathrm{~Hz}$. a) All 150 cycles, and b) cycles from 15 to 20 .

Fig. 6.21 Voltage response of preliminary device design subjected to rolling for a total of 150 cycles at a rolling frequency of $0.25 \mathrm{~Hz}$. a) All 150 cycles, and b) cycles from 15 to 20 .

Fig. 6.22 Typical surface profile of unpatterned $\mathrm{ZnO}$ film after 500 reciprocating cycles with an optical image of the wear track............................... 148

Fig. 6.23 Typical surface profile of 100- $\mu$ m lines sample after various numbers of reciprocating cycles with optical images of the wear tracks.................149

Fig. 6.24 Typical surface profile of $100-\mu \mathrm{m}$ squares sample after various numbers of reciprocating cycles with optical images of the wear tracks.

Fig. 6.25 Typical surface profile of 50- $\mu \mathrm{m}$ lines sample after various numbers of reciprocating cycles with optical images of the wear tracks.

Fig. 6.26 Typical surface profile of 50- $\mu \mathrm{m}$ squares sample after various numbers of reciprocating cycles with optical images of the wear tracks. 
Fig. 6.27 Optical micrographs of 100- $\mu \mathrm{m}$ lines sample before wear testing (left) and after 500 reciprocating wear cycles (right) showing adhesive failure and destruction of the $\mathrm{ZnO}$ line

Fig. 6.28 Optical micrograph of 100- $\mu \mathrm{m}$ square sample before wear testing (left) and after 500 reciprocating wear cycles (right) showing cohesive failure of the $\mathrm{ZnO}$ square structure. 


\section{General Introduction}

Piezoelectric energy harvesting devices have garnered great attention over the previous decade. With advancements in nanotechnology and functional materials, energy scavenging devices may be a key component in solving the ever-growing energy crisis. Numerous device designs have been developed in the scientific community, with significant impetus to increase electrical output of these devices. The crucial consideration of sustained functionality and mechanical reliability of device components has yet to be addressed.

The initial goal for this work was to develop prototypical designs of flexible contactbased energy harvesting devices as a basis for component-level mechanical degradation studies. After successful development of flexible devices based on piezoelectric zinc oxide nanostructures, preliminary mechanical loading experiments demonstrated the fragility of these devices and focus was placed on the two device components with highest likelihood of failure: the conductive electrode and piezoelectric layers. Further aims focused on designing experiments to investigate the durability of these flexible components under mechanical, tribological, and chemical corrosive stresses typically applied during manufacture and use. The underlying theme throughout this work is a push for a bottom-up design approach and establishment of standardized mechanical testing protocols for this difficult to characterize system. A constant push for increased

performance is not typically a stimulus for increased durability. For novel materials and devices to achieve their potential in device applications, component-level investigations must be extensively performed. 


\section{General Background}

As outlined in the introduction, the goal of this research was to investigate the reliability of functional components used in flexible piezoelectric energy harvesting devices. While a large number of device designs have been developed, this work focuses on a simplified, bottom-up approach to device improvement. Focus has been placed on prototypical designs and materials commonly used throughout the field of flexible optoelectronic devices. This chapter provides a general review of literature in the areas of flexible device components and their functional durability.

\subsection{Flexible Electronic Devices}

Flexible devices are currently receiving great interest because they enable exciting new form factors, which can be applied to large areas. They are also lightweight, mechanically robust, and can potentially be manufactured using low-cost roll-to-roll fabrication techniques. Metal oxide transparent films, such as indium tin oxide (ITO) and zinc oxide $(\mathrm{ZnO})$, constitute one of the most important classes of materials that can be used in flexible device applications. This is because their properties can be tuned to cover the whole range from being insulators to being semiconductors and therefore they can be utilized in applications such as displays [1], solar cells [2], thin film transistors [3], sensors $[4,5]$, and energy scavenging devices [6].

\subsubsection{Energy Harvesting Devices}

It is estimated that approximately one-third of the energy consumption in the U.S. is spent to overcome frictional processes [7]. A majority of this loss is attributed to 
mechanical action and heat dissipation [8]. Energy harvesting devices utilize active materials, typically piezoelectrics, to convert ambient energies to useful electricity. Current energy harvesting technologies utilizing cantilever-based systems have been greatly investigated. Some noble recent examples include the work of Chang et al. [9] who reported the use of a $\mathrm{Cu} / \mathrm{ZnO} / \mathrm{ITO} / \mathrm{PET}$ cantilever structure to harvest wind power. Also, Xu et al. [10] have reported design of a cantilever that utilizes an extended auxiliary part for uniform strain distribution and improved energy harvesting efficiency. Work has also been conducted concerning harvesting ambient energy from multiple sources. Kumar et al. [11] have reported on the development of a flexible ZnO-Cellulose nanocomposite that can be utilized to harvest both thermal and mechanical energy. The combined energy output from this device was found to be in the $\mathrm{mV}$ range, which shows promise for the technology. Preliminary data provides strong supportive evidence that $\mathrm{ZnO}$ thin films have important energy-scavenging and tribological properties such as piezoelectricity [12], pyroelectricity [13], and solid lubricity [14], which can be utilized in a device which harvests multiple forms of ambient energy. By controlling the film's surface topography, through processing, and by regulating the rolling contact mechanics involved, the frictional heating and mechanical force output can be effectively managed. There are three main types of mechanical energy harvesting techniques; piezoelectric, electro-magnetic, and electrostatic [15]. Piezoelectric nanogenerators are of particular interest due to their high voltage output. For this reason, a large variety of energy harvesting designs have emerged [16]. 


\subsection{Flexible Substrates}

While substrates comprised of glass or sapphire are general preferred in device fabrication because of added rigidity, flatness, optical clarity, and ease of fabrication, polymeric substrates are the norm in flexible device manufacture, because of their optically clear and mechanically compliant qualities. While flexible substrates provide a platform for novel technologies, they also introduce a number of mechanics of materials issues. Outside of polymer substrates, metal foils and thin glass can be used as substrates for complaint devices, but bring with them additional complications such as susceptibility to cracking, tearing, and internal device short circuiting [17].

Polyester substrates such as polyethylene terephthalate (PET) [18], polyethylene naphthalate (PEN) [19], polycarbonate (PC) [20], and polyesthersulfone (PES) [21] are typically used due to their low cost, flexibility, and mechanical robustness, which also make them an ideal candidate for roll-to-roll processing techniques. Polymer substrates are not without their limitations. Most polymers feature low thermal stability, high permeability to gases and water vapor, are prone to chemical degradation, and have high surface roughness. Polymeric films are typically produced by drawing down an amorphous cast biaxially [22]. Heat treatment is used to crystallize the film and to control film shrinkage. In order to reduce surface roughness and create a smooth platform for fabrication, a planarizing coating is typically applied to one or both faces of the polymer film prior to additional deposition or fabrication steps [22,23]. 
PET and PEN are ideal candidates for roll-to-roll processing due to their relatively high modulus of elasticity, 5.3 GPa and 6.1 GPa, respectively. [24,25] During high temperature roll-to-roll processes above the glass transition temperature, polymer substrates undergo a molecular relaxation process, which alters the physical and mechanical properties of the film [26].

\subsection{Transparent Conductive Oxides}

A majority of commercially available conductive substrates used in flexible optoelectronic devices are comprised of transparent conducting oxide (TCO) layers deposited on relatively thick flexible polymer substrates. Currently, the use of TCO films dominates the market since they offer a combination of high transparency $(>90 \%)$ to visible light and low electrical resistivity $\left(\sim 10^{-4} \Omega \mathrm{cm}\right)$ when deposited on glass substrates at elevated temperatures [27]. By far the most utilized and commercially available TCO film is ITO sputtered at room temperature on polyester substrates such as PET and PEN [28].

\subsubsection{Indium Tin Oxide}

ITO is a wide band-gap (3.5-4.3 eV) n-type semiconductor. It features relatively low electrical resistivity $\left(\sim 7 \times 10^{-4} \Omega \mathrm{cm}\right)$ and a high transparency $(>90 \%)$ to the visible light spectrum $[29,30]$. Electrical conductivity is achieved through a tin-doped $\operatorname{In}_{2} \mathrm{O}_{3}$ lattice. The addition of tin causes the substitution of $\mathrm{Sn}^{4+}$ with $\mathrm{In}^{3+}$, which creates more electrons by means of the n-type donor mechanism [31]. At elevated dopant levels of tin content (>10 mol\%), n-type doping is the dominant factor in raising electrical conductivity, while 
oxygen vacancies play a role in determining the conductivity at lower tin levels [32].

Crystallographic structure of the ITO film determines its level of conductivity. In a highly crystalline form, the semiconducting ceramic contains free electrons generated within the grains and electrons trapped at the grain boundaries [33]. Limited by this transport phenomena, amorphous ITO is less conductive than its crystalline counterpart. Crystal structure is highly dependent on deposition technique. The low temperature sputtering of ITO on flexible polymer substrates often leads to an amorphous microstructure [34]. Thin films of ITO are typically deposited on flexible polymeric substrates using electron beam evaporation, sputter deposition, and PLD methods [34- 39].

\subsection{Piezoelectric Zinc Oxide}

Since the development of zinc oxide ( $\mathrm{ZnO}$ ) films in the 1960's [40], this metal-oxide has spurred an entire field of research dedicated to understanding and utilizing its unique material properties. Further fueled by the recent interest in nanotechnology and nanostructured materials, $\mathrm{ZnO}$ nanostructures have garnered much interest for application in the fields of active sensors and ambient energy harvesters [41-52].

More recent interest can be attributed to the piezoelectric and semiconductive properties that the crystal structures of $\mathrm{ZnO}$ are exhibiting. Wurtzite $\mathrm{ZnO}$ lacks a center of symmetry and features a number of alternating planes composing of tetrahedrally

coordinated $\mathrm{O}^{2-}$ and $\mathrm{Zn}^{2+}$ ions, alternatively stacked along the c-axis, leading to its piezoelectric and pyroelectric properties [42]. 

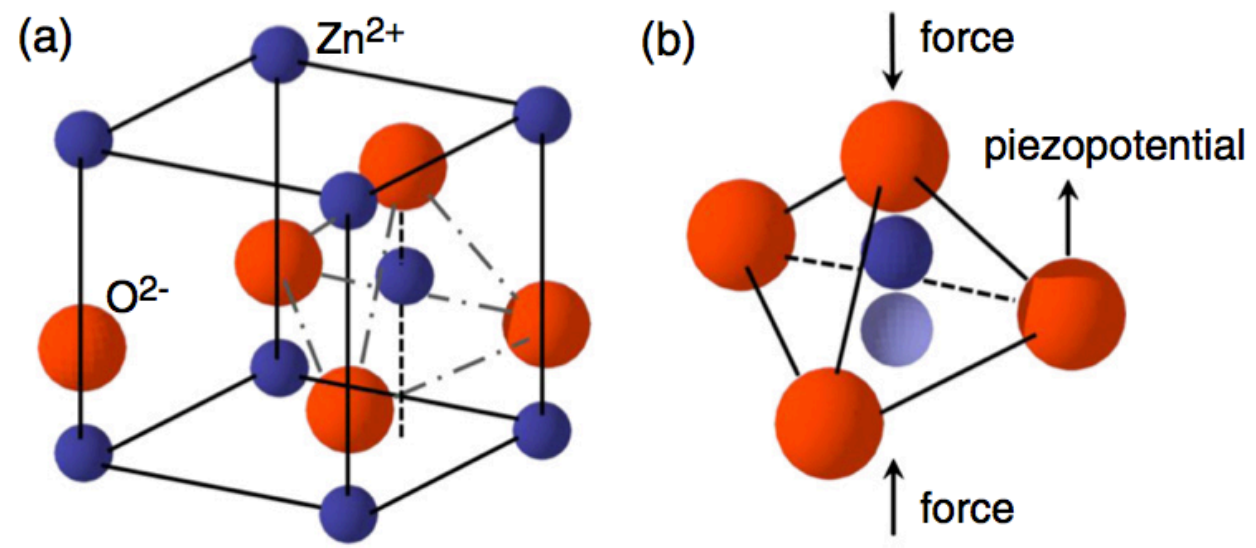

Fig. 2.1 - a) Wurtzite structure of $\mathrm{ZnO}$ b) Generation of a piezoelectrical potential in a $\mathrm{ZnO}$ crystal.[53]

The polar surfaces of wurtzite $\mathrm{ZnO}$ are also an important functional characteristic, especially for controlled growth applications. The oppositely charged ions produce positively charged $\mathrm{Zn}-(0001)$ and negatively charged O-(0001) surfaces. This results in a dipole moment and spontaneous polarization along the c-axis. Zinc oxide also features a wide band gap of $3.37 \mathrm{eV}$ at room temperature and a large excitation binding energy of $60 \mathrm{eV}$, which make it an attractive option for optoelectronic devices [42].

Along with its piezoelectric and semiconductive properties, the diverse range of possible growth geometries and structures makes $\mathrm{ZnO}$ an attractive candidate for device fabrication where shape and size in the nanoscale are both important. By regulating chemistry and growth conditions, the morphology of the resulting nanostructures can be controlled by utilizing rates of growth along the planes and facets of the crystal structure. The different kinetic parameters for each crystal plane of a single crystal can be utilized and controlled to yield nanowire/nanorods, nanobelts with various orientations, and a host of more complex 2-D nanostructures. [40,42-44] 


\subsubsection{Zinc Oxide Films}

$\mathrm{ZnO}$ films are used in a variety of electrical device applications, which include sensors [54], solar cells [2,55], and energy scavenging devices [50,56]. Films can be deposited using pulsed laser deposition (PLD) [2,14,57], chemical vapor deposition (CVD) [58], DC magnetron sputtering [38,54], RF magnetron sputtering [12,13,59], aqueous solution $[56,60]$ and sol-gel $[55,61-65]$. The sol-gel process is a low-cost, large-area and relatively simple solution-based technique.

While sol-gel processing is attractive for its potential for low-cost, large area deposition, $\mathrm{ZnO}$ films deposited via sol-gel coatings must have a c-axis oriented structure to provide a piezoelectric response [64]. This is achieved through careful selection of sol-gel precursor composition and thermal annealing techniques. [61-64].

Another attribute, which make $\mathrm{ZnO}$ films attractive, especially for controlled tribological applications, is the material's surface response to ultraviolet (UV) irradiation [66-69]. Feng et al. investigated the wettability of c-axis oriented $\mathrm{ZnO}$ nanorod films characterized by water contact angle measurements [66]. It was discovered the films exposed to UV light for more than two hours featured a water contact angle near $0^{\circ}$, making the films superhydrophilic. Unexposed films, had a superhydrophobic water contact angle of $150^{\circ}$.

\subsubsection{ZnO Nanostructure Deposition}

\subsubsection{Hydrothermal Growth of Zinc Oxide Nanostructures}

A lower temperature alternative to Vapor-Liquid-Solid deposition was the aqueous chemical-assisted growth process first reported by Vayssieries. [70-72] Vayssieries 
identified the need for a lower temperature process for growth of aligned $\mathrm{ZnO}$ nanostructures, which involved a template-less, and surfactant-free aqueous solution process, which had previously been shown to be successful at growing large arrays $\mathrm{ZnO}$ microtubes. [73] Vayssieries showed that $\mathrm{ZnO}$ could be processed as nanowires or arrayed nanorods by monitoring hydrolysis of $\mathrm{Zn}\left(\mathrm{NO}_{3}\right)_{2}$ in the growth solution.

\subsubsection{Hydrothermal Growth Parameters}

Several studies have investigated the parameters associated with solution growth processing of $\mathrm{ZnO}$ nanostructures. Growth variables include chemical composition of growth solution, growth temperature, time of growth, and seeding of the growth substrate. Manipulation of these variables resulted in a variety of nanostructure geometries, orientations, and densities on the substrate.

Common sources of zinc for solution growth include $\mathrm{Zn}\left(\mathrm{O}_{2} \mathrm{CCH}_{3}\right)_{2}\left(\mathrm{H}_{2} \mathrm{O}\right)_{2}, \mathrm{Zn}\left(\mathrm{NO}_{3}\right)_{2}$ [73-85], $\mathrm{ZnCl}_{2}$ [75,25], and $\mathrm{ZnSO}_{4}$ [60]. Common buffer additives used are hexamethylenetetramine (HMTA) [60,73-78,47-52] and ammonium hydroxide $[81,83]$. Work by Govender et al. [73] studied the effects of different zinc sources and additives, characterizing the geometry of the resulting nanostructures.

Growth temperature predominantly affects nanowire length and diameter [17]. Guo et al. discussed the increase in nanorod length under various constant growth temperatures between $40^{\circ} \mathrm{C}$ and $95^{\circ} \mathrm{C}[80]$. Results showed that nanorod length and growth temperature have a linearly proportional relationship, as the longest nanorods observed at 
$95^{\circ} \mathrm{C}$. Growth temperature was also believed to influence the tip geometry of the nanostructures. Work by Tak et al. [76] found that $\mathrm{ZnO}$ nanowires grown at $90^{\circ} \mathrm{C}$ exhibited a more faceted and broader tip when compared to nanowires grown at lower temperatures. Li et al. [77] found that the diameters of nanowires more than doubled when decreasing the temperature from $98^{\circ} \mathrm{C}$ to $92^{\circ} \mathrm{C}$. Xu et al. [60] determined that the optimum growth temperature for $\mathrm{ZnO}$ nanowires, derived from a zinc nitrate and HMTA solution, was equal to $70^{\circ} \mathrm{C}$. They cite the density, aspect ratio, and alignment were optimal at $70^{\circ} \mathrm{C}$ when compared to growth conditions with lower or higher temperatures.

In addition, growth time has been shown to primarily affect nanowire length and diameter. Guo et al. reported that during the early stages of growth, nanowire growth is dominated by lateral growth for up to six hours [74]. After this initial lateral growth regime, the nanostructure growth is dominated by expansion along the major axis. Li et al. [77] investigated the geometric structure of $\mathrm{ZnO}$ nanostructures after various growth times. After ten hours of growth, nanorods were observed. As the growth times were increased above ten hours, nanotubes were observed to grow from the tips of nanorods. These secondary growth structures had an average diameter of $50 \mathrm{~nm}$. After 20 hours of growth, these secondary nanotube structures were much longer and began to entangle.

Although growth of $\mathrm{ZnO}$ nanowires is possible on many substrates, to obtain vertical alignment, the substrate must be smooth. [81] Smoothness of the growth substrate is important, while substrate crystallinity has been shown to have no effect on aligned growth. [73] This allows growth of vertically aligned nanowires on both rigid and 
flexible substrates, but increased the importance of how the substrates are prepared for growth.

Seeding of the smooth substrate controls nucleation sites and influence the orientation and aspect ratio of the $\mathrm{ZnO}$ nanowires. A range of seeding techniques were implemented into growth processes to provide more control of the resultant nanowires. Work by Chen et al. [82] implemented sol-gel deposited $\mathrm{ZnO}$ films on bare rigid substrates, which were then sintered at various temperatures. Firing temperatures below $200^{\circ} \mathrm{C}$ yielded poorly aligned rods compared to those grown on seed layers fired at higher temperatures. Additionally, seed layers fired at higher temperatures yielded nanowire/rods with larger diameters. Song et al. [81] investigated $\mathrm{ZnO}$ seed layers doped with aluminum and gallium and observed varying nanowire densities, diameter, and growth rates, which were heavily influenced by the composition of the seed layer.

She et al. [86] investigated the use of seed layers not based on $\mathrm{Zn}$. $\mathrm{ZnO}$ nanowires were grown on ITO coated glass with a seed layer of Au which was found to produce more densely packed arrays of nanowires than that without the gold seed layer. Further work by Govender et al. [73] expanded on the use of Au as a seeding layer by depositing it on fluorine doped tin oxide glass substrates. It was observed that the seed layer influenced the diameter of the rods, but the growth time and length were not significantly affected.

\subsection{Mechanical Properties of Thin Film Electrodes}

When ITO-coated PET is tested in tension, cracks form on the ITO surface with their 
orientation being perpendicular to the tensile direction at around $2 \%$ uniaxial tensile strain. The onset of cracking results in an abrupt increase of the ITO electrical resistance, which in turn initiates device degradation [87]. In situ optical observations of crack initiation and formation correlates well with in situ electrical resistance measurements [88]. Furthermore, damage due to monotonic buckling of ITO-coated PET substrates has been reported to be even lower than $2 \%$ [89]. In addition to catastrophic failure when a critical strain is reached, TCOs are also susceptible to damage accumulation during cyclic repetitive loading at lower than $2 \%$ strains [90]. Cyclic loading under controlled buckling conditions has also been conducted and shows that the ITO electrical resistance is increasing after each cycle and that there is no significant resistance decrease during the unloading part of each cycle [91].

Cairns et al. [92] reported on the increase in electrical resistance of ITO-coated PET flexible electrodes with increasing tensile strain. Coating cracking and delamination beyond the $2.5 \%$ externally-applied tensile strain threshold were observed. In addition, multiple deformations of the flexible device may result in reduced reliability and, subsequently, in functional failure. It was reported that the use of ITO-coated PET electrodes shows significant disadvantages, in mechanical flexibility terms, as compared to poly (3,4-ethylenedioxythiophene) poly(styrenesulfonate) (PEDOT:PSS) - based anodes $[92,93]$.Also, the mechanical integrity of a flexible system is influenced by the deformation mode applied to it and can be more/less breached when the electrode material is under tension/compression [93]. In the particular case of contact-based force flexible sensors the tribological characteristics of the top surface are also important. Sierros and Kukureka [94] investigated the wear and friction behavior of PET and PEN 
for use as the contact surface in flexible touch-screen devices. PEN was found to exhibit better tribological properties than PET, when it slid against a steel ball counterface. Such behavior was attributed to its relatively low surface roughness. In some cases the device performance can be enhanced by careful materials selection as in the case of selecting mechanically robust carbon nanotube-based electrodes for contact applications [95-97]. Furthermore, important thin-film mechanical deformation modes are bending and controlled buckling under uniaxial stress states. Bouten [88] investigated the failure of ITO on polycarbonate substrates using the two-point bend test, originally developed for optical fiber characterization, coupled with electrical resistance monitoring. It was determined that ITO layers, $100 \mathrm{~nm}$ thick, can fail at a critical failure strain at about $1.2 \%$. The minimum strain at which cracking occurs is a function of both film thickness and quality. Coating defects and particulate matter can act as crack initiators thus reducing the critical strain.

Recently, the need for improved corrosion resistance of the ITO coatings, used in solar cells, was documented [98]. It has been also reported that when ITO anodes are exposed to voltage differences in the presence of moisture and contamination, they are also sensitive to corrosion [99]. Also, it is reported that stress corrosion cracking of transparent conductive layers of ITO, sputtered on PET substrates, is an issue of paramount importance in flexible optoelectronic devices. These components, when used in flexible device stacks, can be in contact with acid containing pressure-sensitive adhesives or with conductive polymers doped in acids. Acids can corrode the brittle ITO layer, stress can cause cracking and delamination, and stress corrosion cracking can cause more rapid failure than corrosion alone [100]. 


\subsection{Tribo-Mechanical Properties of Zinc Oxide}

Thin film $\mathrm{ZnO}$ structures are widely used in cantilever-based, energy harvesting devices. However, the interesting tribological properties that are exhibited by $\mathrm{ZnO}$ films have been little explored with respect to the potential energy harvesting device designs. Prasad et al. $[14,57]$ have reported on the tribological behavior of $\mathrm{ZnO}$ films. They observed a noteworthy wear life, of 500 micron thick films deposited on steel using PLD, equal to $10^{6}$ cycles. They also reported relatively low coefficients of friction for room temperature deposited $\mathrm{ZnO}$ films equal from 0.15 - 0.2. They attributed such lubricity of the $\mathrm{ZnO}$ films to their nanocolumnar and piezoelectric (002) microstructure. In addition, Lin et al. [63] reported on the wear resistance and friction coefficients of RF-magnetron sputtered $\mathrm{ZnO}$ films on glass. Samples that were deposited at room temperature exhibited relatively low friction coefficients and high wear resistance but they were not piezoelectric. The hardness of the films exhibited thickness-dependence. It is important therefore to note the need for relatively high sputtering temperatures and possible post-modification of the resulting $\mathrm{ZnO}$ surface.

Goto et al. [101, 102] reported on the importance of $\mathrm{ZnO}$ tribological properties for microelectromechanical systems (MEMS) applications and discussed the integration of piezoelectric $\mathrm{ZnO}$ films in movable MEMS components in order to decrease the friction force by controlling the electric interaction at the sliding interface. They also reported that the frictional property of $\mathrm{ZnO}$ coatings, sliding against a 'single asperity' stainless steel probe ball (diameter equal to $3.18 \mathrm{~mm}$ ) at atmospheric pressure, satisfies the Amonton's-Coulomb law for loads as high 0.8 N. Amonton's-Coulomb law states that the 
sliding friction force increases linearly with the load [103]. The potential of piezoelectric $\mathrm{ZnO}$ to be used reliably as a tribological material combined with its adequate compatibility to selected micromachining processes [104] and its pyroelectricity [12] makes it an ideal candidate for integration to various rolling/sliding interfaces as well as for a new generation of energy harvesting devices. Such devices can operate in rolling contact motion-in order to limit wear-related degradation- and they will be able to target the harvesting of both mechanical energy originating from the contact pressure and frictional heating originating from the rolling interface.

Previous studies have demonstrated the ability to control the nano-scale sliding friction of piezoelectric $\mathrm{ZnO}$ films by modulation of their surface roughness via chemical etching [105]. It has been hypothesized that patterning of the $\mathrm{ZnO}$ films into structures on the micron-scale can allow control of the piezoelectric and pyroelectric output. Unfortunately, patterning of this functional oxide film introduces tribological difficulties concerning the durability of the patterned films. With combined rolling/sliding contact, the $\mathrm{ZnO}$ structures experience stresses not observed in continuous, pristine films. The electromechanical properties of $\mathrm{Al}$ doped $\mathrm{ZnO}$ films deposited on PET in uniaxial tension were found to be similar to ITO-coated PET [106] with critical strains of $2 \%$. Recently, the fracture behavior of relatively thick Al doped $\mathrm{ZnO}$ films $(1 \mu \mathrm{m}$ in thickness) during simply-supported bending were investigated [107]. It was found that the films fail at strains as low as $0.5 \%$ when the film surface is under tension and at around $1.38 \%$ strain when the film surface is under compression. Nanoindentation and nanoscratch measurements of $\mathrm{Al}$ doped $\mathrm{ZnO}$ on rigid glass substrates indicate a hardness 
of $8 \mathrm{GPa}$ and an elastic modulus of $110 \mathrm{GPa}$ [108]. We report on the deposition and mechanical characterization of $\mathrm{ZnO}$ thin films over a broad thickness range suitable for flexible devices ( $25 \mathrm{~nm}$ to $1 \mu \mathrm{m}$ ). We have focused on the in situ buckling behavior and scratch resistance of films because of the importance of these properties to designing robust devices and developing suitable handling processes for fabrication. Films have been deposited on PET and PEN substrates which are most widely used in flexible device applications.

\subsection{Mechanical Properties of Zinc Oxide Nanowires}

A number of studies have been performed to characterize the mechanical properties of $\mathrm{ZnO}$ nanostructures of various geometries and dimensions. A general consensus in the literature has been drawn to a strong size effect on the modulus of elasticity of nanowires in the [0001] direction. In particular, as the diameter of nanowires decreases below 100 $\mathrm{nm}$, the modulus increases. [50,109]

A number of experimental and theoretical studies have been attempted to measure and characterize the elastic properties of $\mathrm{ZnO}$ nanowires as well as their fracture strength and failure strains. In situ SEM uniaxial $[110,111]$ and buckling experiments $[112,113]$ on individual nanowires confirmed this size dependence and identified an inverse relationship between nanowires diameter and elastic modulus.

While the elastic regime properties of $\mathrm{ZnO}$ nanowires have been well studied, data with regards to fracture strength and strain is limited. Three-point bending experiments on $\mathrm{ZnO}$ nanowires were carried out by Wen et al. using an AFM tip to produce lateral 
bending [113]. Uniaxial fracture tests were more recently performed using in situ TEM techniques by Agarwal et al. [110] From these studies, a size dependence on fracture strength was also found as the fracture strength of $\mathrm{ZnO}$ nanowires of various diameters was found to be a few GPa. Similarly, fracture strains greater than $2 \%$ were observed.

Surface and point defects (vacancies) were cited as the two possible mechanisms for failure of these nanostructures. Agarwal et al. correlated strength and surface imperfections, observing that the imperfections acted as stress concentrators that lead to fracture. [110] This also leads to a dependence on surface area, since the decreased diameter, and therefore the decreased surface area and number of possible surface imperfects, lead to increased failure strength. Another study by He et al. [112] discussed the failure mechanism associated with vacancies in the nanowires themselves, Pugno et al. first developed this mechanism for application on CNTs [114,115]. Application of this mechanism to $\mathrm{ZnO}$ nanowire was performed using Weibull statistics and experimental TEM data. Although the results are suggesting a correlation between fracture and vacancy density, the presence of surface defects was not considered, leading to some debate and inconclusive results [115].

\subsection{Experimental Methods}

\subsubsection{Nanoindentation}

Spherical indentation cracking experiments and film hardness measurements were 
performed on a CSM Instruments NHT ${ }^{2}$ nanoindentation system. Berkovich and $10 \mu \mathrm{m}$ sphero-conical diamond tips were used and a penetration depth was set to $10 \%$ of the coating thickness to minimize substrate effects.

\subsubsection{Scratch Testing}

Scratch testing was performed using a CSM Instruments Nano Scratch Tester (CSM Instruments). Samples were glued on glass microscope slides and mounted to the scratch tester's stage prior to analysis. A diamond indenter with a $5 \mu \mathrm{m}$ radius was drawn across the sample surface with a loading rate equal to $5 \mathrm{mN} / \mathrm{min}$. A total scratch length of 0.5 $\mathrm{mm}$ was generated with the end load being $5 \mathrm{mN}$. The failure modes that occur at some critical loads were confirmed using a built-in optical microscope. Also, a built-in atomic force microscope (AFM) operating in tapping-mode was used to observe the film's scratched areas.

\subsubsection{Controlled Buckling}

Controlled buckling experiments of ITO and $\mathrm{ZnO}$ films were conducted using a custombuilt, manually operated apparatus (Fig. 2.2). 


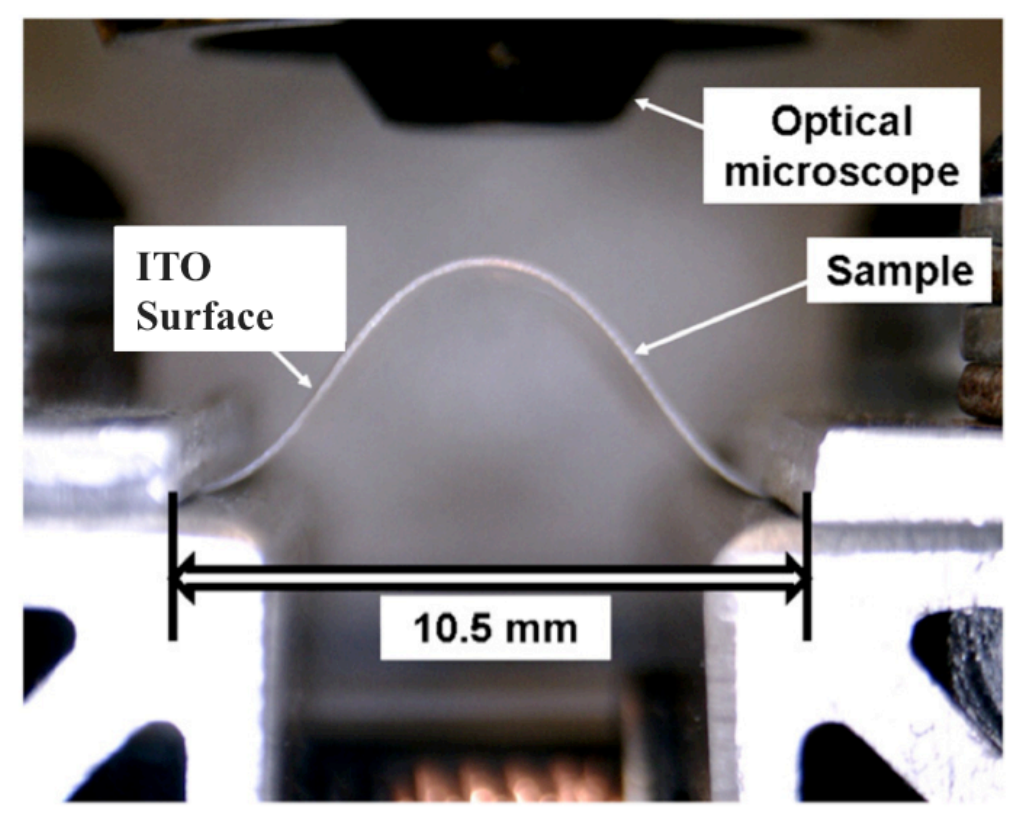

Fig. 2.2 - Side-view of custom-built controlled buckling experimental apparatus.

Opposing ends of the flexible samples were securely clamped using aluminum grips. The apparatus was mounted beneath an optical microscope which was equipped with a frame grabber (Guppy, Allied Visions Technology). Sample displacement measurements were taken using a side-view digital imaging system (Celestron Digital Microscope) and they were analyzed using image analysis software (ImageJ NIH USA). The buckling radius and the resulting strain were calculated according to the procedure described by Chen $e t$ al. [89]. In situ optical microscopy images were taken incrementally at various applied strains to determine both tensile and compressive crack onset strain values and to monitor crack propagation. In situ electrical resistance changes were monitored using a Fluke 45 dual display multimeter.

\subsubsection{Tensile Testing}

ITO-coated PET samples were tested in uniaxial tension with in situ electrical resistance monitoring using a commercial mechanical testing machine (Instron 4410). Typical 'dog- 
bone' specimens with gauge length of $25 \mathrm{~mm}$ and width of $5 \mathrm{~mm}$ were used and the operating crosshead speed was $0.3 \mathrm{~mm} / \mathrm{min}$. ITO electrical resistance data were recorded during the tests using an Agilent 349708 data acquisition/switch unit.

\subsubsection{Electrochemical Impedance Spectroscopy}

Electrochemical measurements were performed on ITO-coated PET samples with resistivity equal to $100 \Omega /$ sq. The samples were washed with distilled water and dried before performing the electrochemical measurements. Platinum gauze was used as the counter electrode and a saturated $\mathrm{Ag} / \mathrm{AgCl}$ electrode was employed as the reference

electrode. The ITO sample area exposed was $1 \mathrm{~cm}^{2}$. Electrochemical impedance spectroscopy (EIS) measurements were conducted using an impedance analyzer (Solartron Analytical, 1260) connected to a potentiostat (Solartron Analytical, 1287). A stable open-circuit potential was attained before performing the EIS measurement. EIS spectra were recorded over a frequency range of $10^{6}$ to $0.01 \mathrm{~Hz}$ with applied AC amplitude of $10 \mathrm{mV}$.

\subsubsection{Time-of-Flight Secondary Ion Mass Spectrometry}

High-resolution TOF-SIMS analysis was performed with a TFS series instrument (Charles Evans \& Associates, Redwood City, CA), using a $69 \mathrm{Ga}^{+}(15 \mathrm{keV})$ liquid metal ion source. Test coupons were prepared with a $9.5 \mathrm{~cm} \times 2 \mathrm{~cm}$ strip of PSA adhered to the center of a $9.5 \mathrm{~cm} \times 9 \mathrm{~cm}$ ITO-coated rectangle of PET. SIMS depth profiling was performed through the pressure sensitive adhesive (PSA) and ITO layers. 


\subsubsection{Stress-Corrosion Cracking Investigations}

Mandrels made from chlorinated polyvinyl chloride (c-PVC) of various diameters were utilized to apply various bending strains to sample strips of ITO-coated PET. A diagram of the stress corrosion cracking experimental procedure is found in Fig. 2.3.

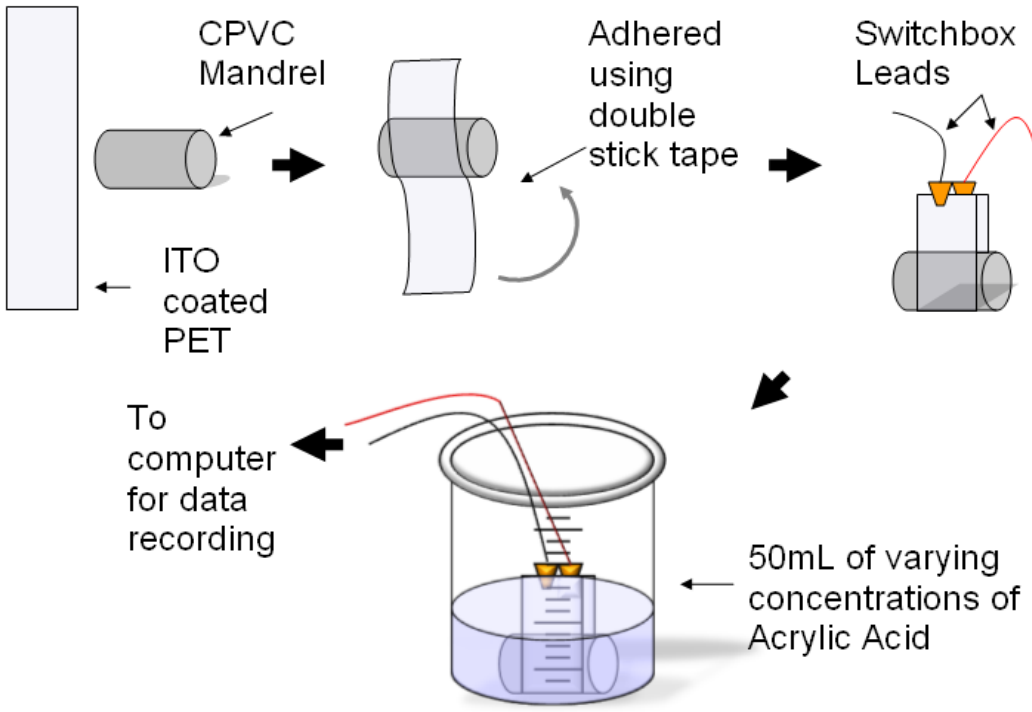

Fig. 2.3 - Experimental schematic of stress-corrosion cracking apparatus.

The strips were adhered around the center of the mandrels using double-sided adhesive tape. Small strips of aluminum foil, approximately a centimeter in width, were wrapped around each end of the sample where leads, for electrical resistance monitoring, are to be attached. This electrically-transmissive buffer between the applied lead and the ITO surface allowed for a better electrical connection and minimal damage to the ITO conductive surface. The leads were connected to a digital switchbox that allowed for multi-channel data logging (Agilent 349708 Data Acquisition/ Switch Unit) and were then attached to opposite sides of the ITO coated PET strip. The wrapped mandrel was then dipped into various $50 \mathrm{ml}$ solutions of acrylic acid of varying concentration. 


\subsubsection{Reciprocating Wear Testing}

Counterformal contact under pure dry, and wet, sliding conditions was achieved by means of a custom-built reciprocating wear tester (Fig. 2.4).

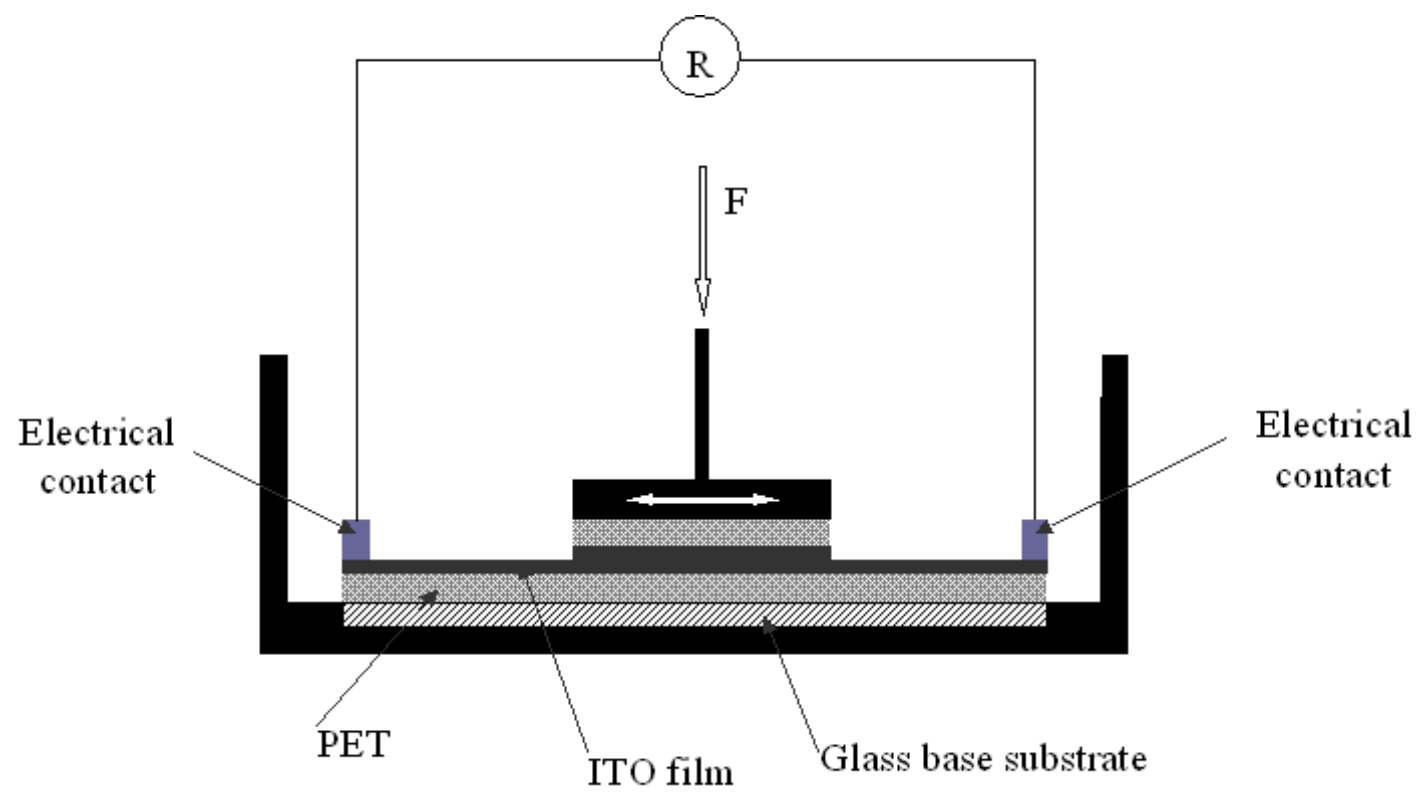

Fig. 2.4 - Schematic of custom-built reciprocating wear apparatus.

The bottom counterface sample, $36 \mathrm{~cm}^{2}$ area, was adhered to a glass base substrate using a double-sided adhesive tape. Particular care was taken when adhering the sample to the glass substrate to avoid premature cracking of the brittle ITO coating. The sample fit inside a plastic container. Under wet sliding conditions, the container was filled with acrylic acid of $0.1 \mathrm{M}$ concentration. The top ITO-coated PET surface, $12 \mathrm{~cm}^{2}$ area, slid in a reciprocating fashion against the bottom surface. The top sample was adhered to the top metal surface using double-sided adhesive tape. The electrical resistance of the top ITO surface was measured before and after testing. A constant normal load $F$ equal to 3.5 $\mathrm{N}$ was applied. At each end of the bottom conductive surface a metal bar was placed to act as an electrical contact for in situ electrical resistance, $R$, monitoring of the bottom ITO surface. Electrical resistance data were recorded during testing using an Agilent 
349708 data acquisition/switch unit. Each test was conducted using a fixed reciprocating speed of 34 cycles/s. Wear tests, in dry and wet conditions, were run for up to a few thousand reciprocating cycles. Each reciprocating cycle was equal to $50.8 \mathrm{~mm}$ of linear sliding distance. Two samples were tested at each set of conditions.

\subsubsection{Line-Contact Rolling/Sliding Experiments}

Macrotribological experiments were performed using a custom-built reciprocating wear tester, shown in Fig. 2.5.

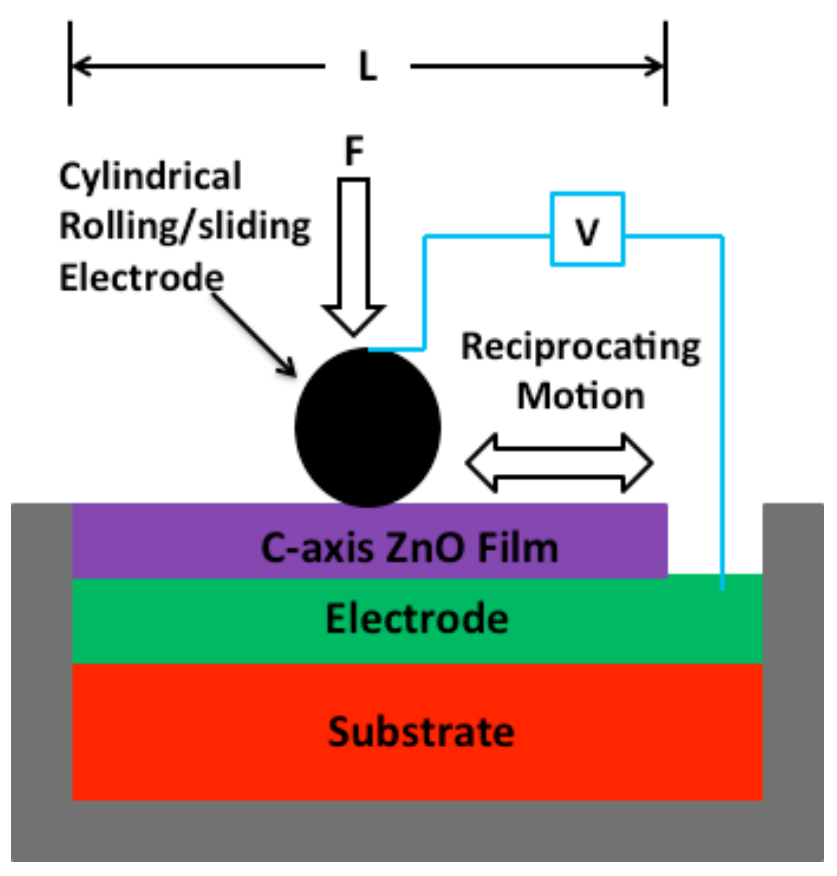

Fig. 2.5 - Schematic of custom-built reciprocating rolling-contact experimental apparatus with in situ electrical monitoring.

Samples were placed on a custom-built holder, to avoid clamping complications. The counter-surface used was a stainless steel cylinder, with dimensions of $15.9 \mathrm{~mm}$ in diameter and $25 \mathrm{~mm}$ in length. The cylinder performed a mixed rolling-sliding motion in a reciprocating fashion along the sample's surface. The reciprocating length was equal to 
$35 \mathrm{~mm}$. Stainless steel was used because of its moderate coefficient of friction and its structural rigidity. A constant, relatively moderate, load $\mathrm{F}$ equal to $2.5 \mathrm{~N}$ was applied normally to the reciprocating direction. The samples were tested in increments of reciprocating cycles until the coating was totally removed. The rolling frequency was set equal to $0.5 \mathrm{~Hz}$ for all tests. In addition, tests were performed on samples both exposed and non-exposed to UV light. The electrical output of the piezoelectric devices was monitored in situ using the previously described digital multimeter.

\subsubsection{Point-Contact Rolling/Sliding Experiments}

Combined rolling/sliding contact of the 302 stainless steel ball and the $\mathrm{ZnO}$ bottom counterface was achieved using a custom-built reciprocating wear tester. A custom ball holder was fabricated with a stainless steel cup to retain the ball, while allowing rolling and sliding translation of the ball during reciprocation. A constant normal load of $2.3 \mathrm{~N}$ was applied. Each test was conducted using a fixed reciprocating speed of $0.1 \mathrm{cycles} / \mathrm{sec}$. Each reciprocating wear cycle was equal to $18 \mathrm{~mm}$ of linear sliding distance. Wear tests were run up to five hundred cycles. Fig. 2.6 shows a representation of the custom build reciprocating wear apparatus. 


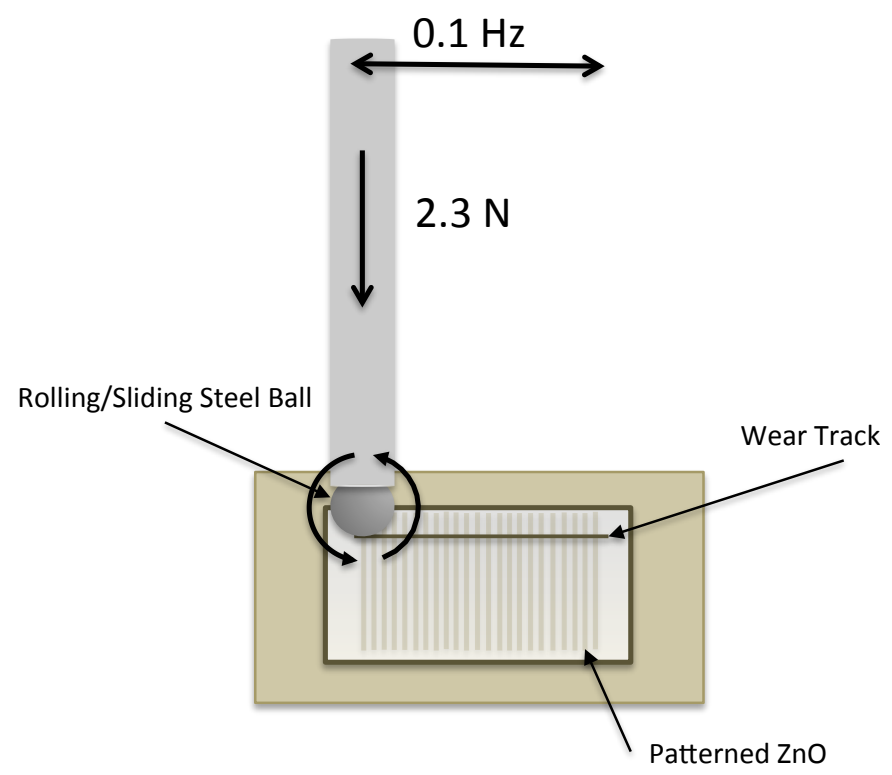

Fig. 2.6 - Schematic of reciprocating wear apparatus and ball bearing holder used in this study.

Weight measurements on the $\mathrm{ZnO}$ counterfaces were conducted before and after wear cycles using a digital balance (DV215CD, Ohaus, $0.01 \pm 0.02 \mathrm{mg}$ ). Between testing and weighing, the samples were washed in isopropyl alcohol, deionized water, and dried on a hotplate at $120^{\circ} \mathrm{C}$.

\subsubsection{Scanning Electron Microscopy}

The surface morphology of films was characterized using a JEOL JSM-7600F scanning electron microscope (SEM). Before SEM investigation, specimens were sputter-coated with a $5 \mathrm{~nm}$ thick Au layer using a Cressington 108 sputter coater.

\subsubsection{X-Ray Diffraction}

$\mathrm{X}$-ray diffraction $(\mathrm{XRD})$ was used to characterize the crystallographic orientation of oxide films and nanostructures. Analysis was performed on a Bruker D8 Discover 
diffractometer with a $\mathrm{Cu}-\mathrm{K} \alpha$ source, $\lambda=0.151417 \mathrm{~nm}$. Emmison was set to $40 \mathrm{~mA}$ and 40 $\mathrm{kV}$, and a $2 \theta$ step size of $0.01^{\circ}$ and time/step of $1 \mathrm{~s}$ were used.

\subsubsection{Contact Angle Gonionmeter}

Static contact angle measurements were performed to characterize the wetting properties of various films. Five drops $(\sim 2 \mu \mathrm{L})$ of deionized water were placed on the sample's surface. An image of the 5 droplets was taken using a fixed microscope. The image was digitally analyzed using the Image J “drop analysis” plugin method LB-ADSA.

\subsubsection{Atomic Force Microscopy}

Atomic force microscopy (AFM) was conducted using a Molecular Imaging PicoScan 3000 system in contact mode with silicon tips on cantilevers.

\subsubsection{Stylus Profilometry}

Film roughness and tribological wear tracks were measured on a Veeco Dektak 150 stylus profilometer. 


\section{Fabrication and Characterization of Flexible Piezoelectric Nanogenerators}

\subsection{Overview}

This chapter focuses on process development for prototypical flexible energy harvesting devices based on a piezoelectric zinc oxide active layer. The designs for the model devices were based on those previously reported in the literature. Model devices based on glass and polymeric substrates were successfully fabricated and characterized in three phases. The limited sustained durability observed during testing was the impetus for investigations in further chapters, because it is believed a component-level, bottom-up design approached is needed before this novel technology field can reach market acceptance. Results from this study were presented at the 2013 Flexible and Printed Electronics Conference.

\subsection{Introduction}

In 2004, Zhao et al. realized the potential for $\mathrm{ZnO}$ nanostructures to be used in energy scavenging devices [116]. The study found the piezoelectric coefficient of a single $\mathrm{ZnO}$ nanobelt to be 2-3 times the bulk. This was the first time the nano-scale piezoelectric property was explored. Further work by Wang et al. used and a contact mode AFM with a conductive tip to measure the electrical output associated with mechanical bending of an individual $\mathrm{ZnO}$ nanowires. A constant force of $5 \mathrm{nN}$ was applied as the tip scanned across the terminal ends of the $\mathrm{ZnO}$ nanowires, causing their deflection and subsequent relaxation as the AFM tip moved across the sample. For nanowires of diameter 30-40 nm and length 1-2 $\mu \mathrm{m}$, the output was measured to be 3-12 $\mathrm{mV}$ [117]. 


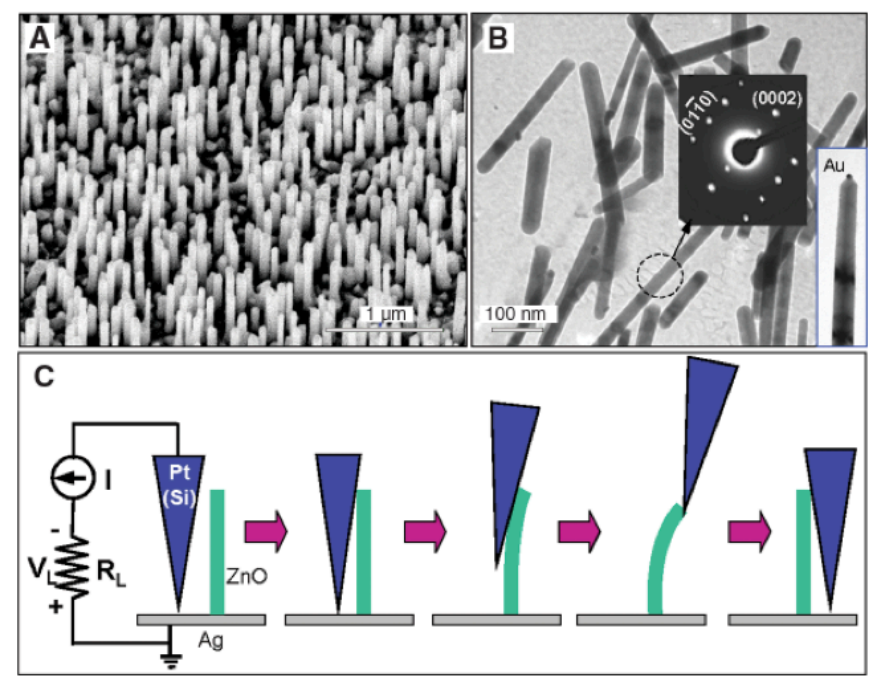

Fig. 3.1 - a) SEM micrograph of vertically-aligned $\mathrm{ZnO}$ nanowires b) TEM images of $\mathrm{ZnO}$ nanowires c) Experimental setup for measuring piezoelectric output of individual $\mathrm{ZnO}$ nanowires using a conductive AFM tip [117].

This concept was the initial impetus for numerous subsequent designs of active sensing and ambient energy harvesting devices utilizing $\mathrm{ZnO}$ nanowires. With novel designs being reported in the literature at a rapid pace, concern for maximizing the electrical output remained the primary concern. Although early works by Xu et al. presented a maximum voltage output of $65 \mathrm{mV}$, several years of iterative design, have led to device outputs above $20 \mathrm{~V}$. [52]

This impressive increase in electrical output is exciting for the field of ambient energy harvesting, but the sustained reliability of these devices has yet to be addressed. Although, the mechanical properties of single $\mathrm{ZnO}$ nanostructures have been investigated, it is difficult to characterize the material properties of these nanostructures under various mechanical loading conditions, especially when they are grown in largearea arrays. The addition of several other device elements, such as flexible polymeric 
substrates and thin film electrodes only further complicates the system and introduces several possible failure scenarios.

After considering all variables associated with the hydrothermal growth of $\mathrm{ZnO}$ nanowires, the current energy harvesting devices based on $\mathrm{ZnO}$ nanowires were analyzed to develop the best model system for mechanical testing. The initial designs of nanogenerator systems were based on $\mathrm{ZnO}$ nanowires grown on Si substrates. A top zigzag shaped, gold electrode was fabricated to mimic the deformation previously achieved by the scanning AFM tip, with the goal to activate numerous nanowires simultaneously, shown in Fig. 3.2. The mechanical strain was applied by externally-driven ultrasonic vibrations at a frequency of $41 \mathrm{kHz}$. The electrical response was measured in situ [85].

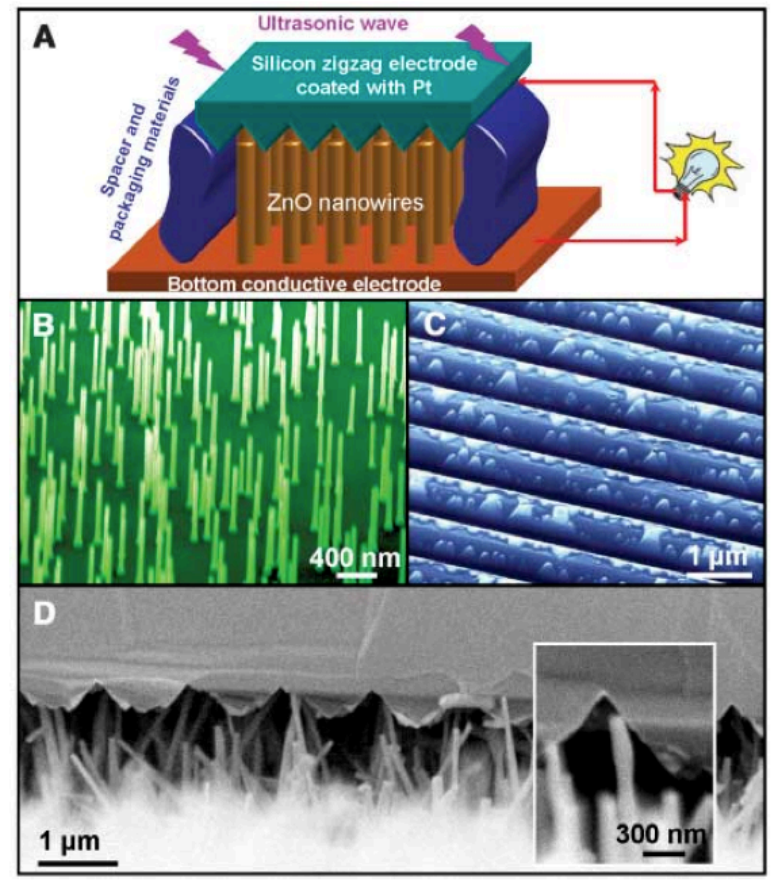

Fig. 3.2 - a) Schematic of vertically-aligned nanogenerator device with zigzag silicon top electrode b-d) SEM images of various device components. [85] 


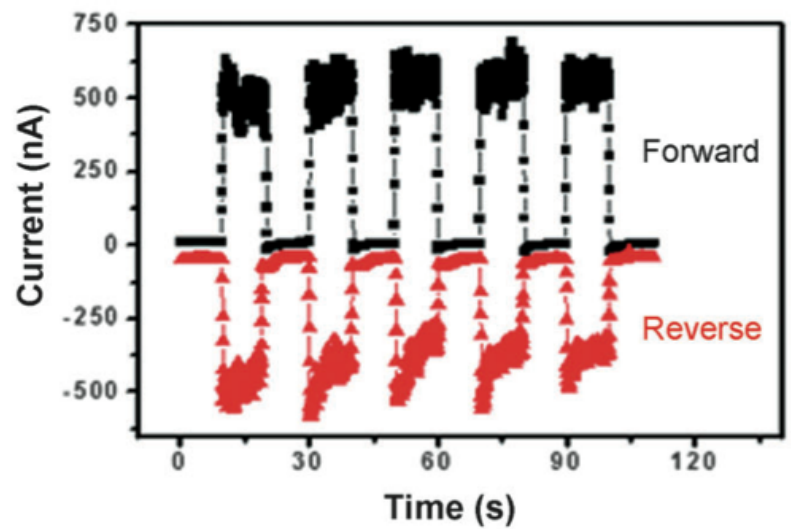

Fig. 3.3 - Current output of the zigzag substrate device under ultrasonic wave loading [85].

In order to increase the output voltage and current of the device, a stack of these elements were integrated in parallel/series, and an output voltage of $65 \mathrm{mV}$ was measured. [46] This device design was significant, in that, it was the first design of its kind utilizing vertically aligned $\mathrm{ZnO}$ nanowires sandwiched between two conductive electrodes, which proved to be the model of future designs.

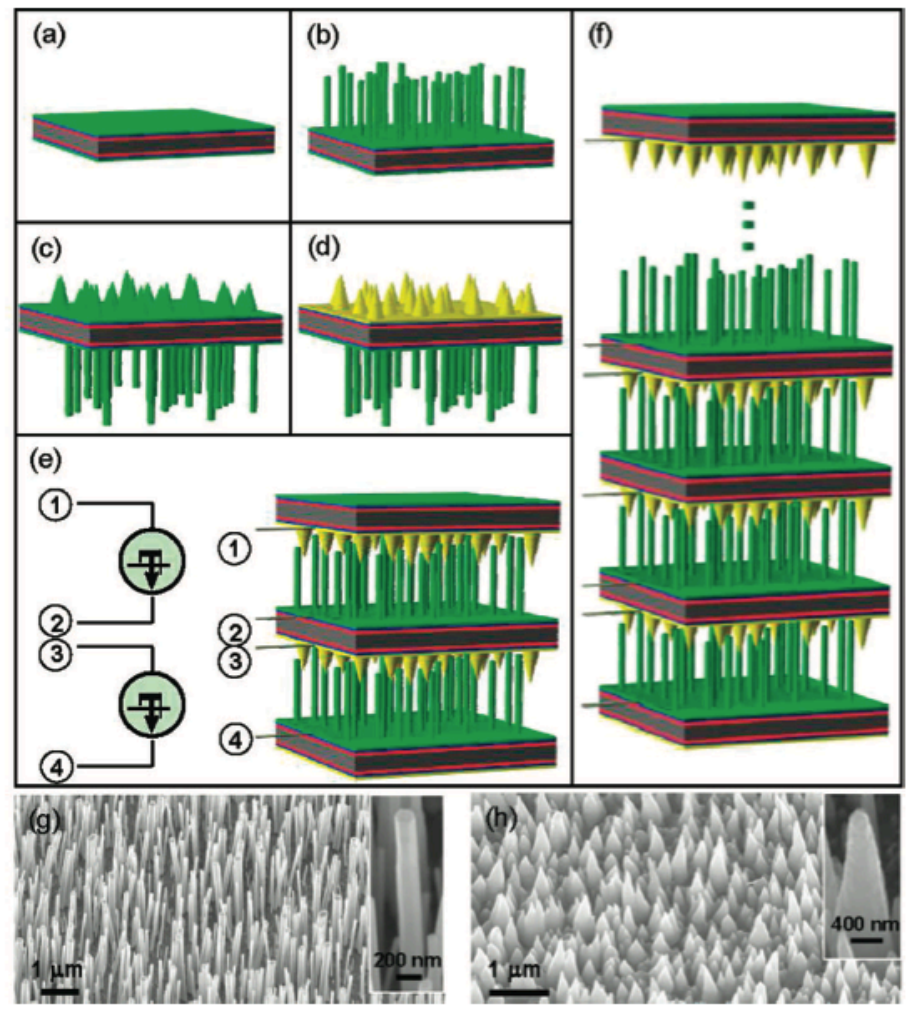

Fig. 3.4 - Design and fabrication procedure of multilayered nanogenerator with SEM images of ZnO nanowires and gold-coated conical counterface [46]. 
Laterally aligned nanogenerator devices also represent an important design. Identifying a mechanical advantage associated with the shift to laterally aligned nanowires, Yang et al. developed a nanogenerator based on a single $\mathrm{ZnO} \mathrm{NW}$ laterally positioned along a Kapton substrate [44]. Terminal ends of the nanowires were affixed using metal electrodes. To generate an output, the Kapton film was mechanically bent and released using a motor driven mechanical arm to a final radius of $\sim 2 \mathrm{~cm}$. This repeated tensile strain of $0.05-0.1 \%$ in the NW generated a maximum transient voltage up to $\sim 50 \mathrm{mV}$. Further work by Yang et al. showed the potential use of this device design to harvest low-frequency mechanical energy from both ambient environmental energy, as well as energy from a biological entity. [44].

(a)
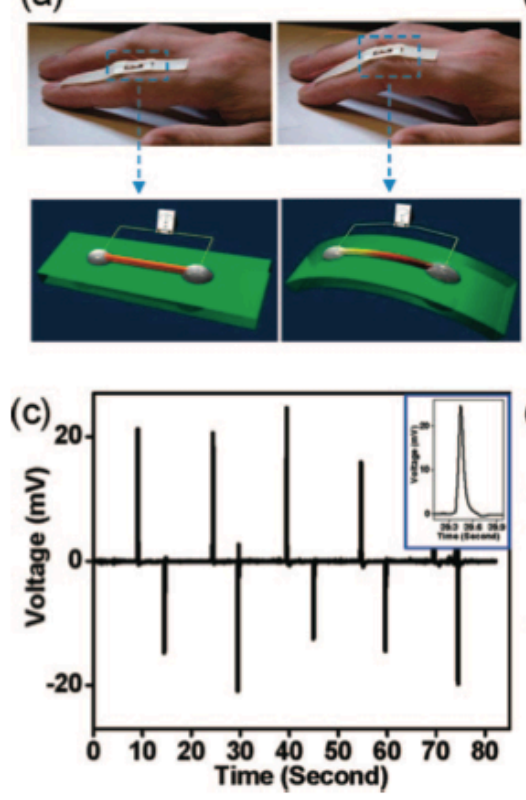
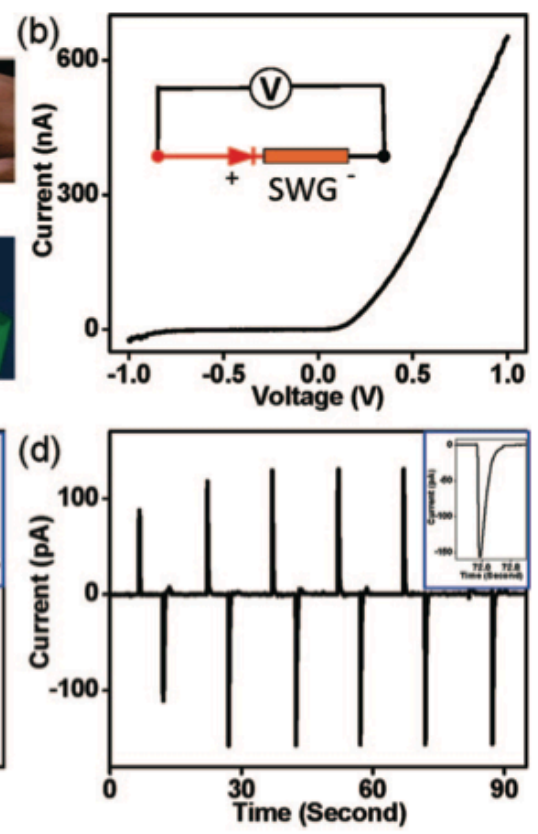

Fig. 3.5 - a) Single-wire nanogenerator device and piezoelectric potential modeling b-d) Current and voltage output of single-wire nanogenerator device under oscillating fingertip bending. [44]

The advantage of laterally-aligned nanowires was scaled up from a single nanowire design the use of arrays for increased output. [45-47]. This design yielded a very high open-circuit voltage output of $2.03 \mathrm{~V}$ and a peak output power density of $11 \mathrm{~mW} / \mathrm{cm}^{3}$. 
More recently, $\mathrm{Hu}$ et al. further developed the field of laterally-aligned nanowires by growing conical nanowires and freely suspending them in a PMMA matrix. $[48,49]$. After integrating this "composite" between two parallel plate electrodes and subjecting it to a mechanical contact load, an open-circuit output of $2 \mathrm{~V}$ and a closed-circuit current of $50 \mathrm{nA}$ was measured. This was cited as enough power to drive small electronic devices such as liquid crystal displays.

Work by Wang et al. further maximized the output voltage by using vertically aligned $\mathrm{ZnO}$ nanowires grown on both sides of a $\mathrm{Cr} / \mathrm{Au}$ coated polystyrene (PS) film. [83]. The dielectric matrix of PMMA was used to fill the volume around the nanowires before top electrodes were deposited. By introducing a strain of $0.12 \%$ at a rate of $3.56 \% / \mathrm{s}$, a measured voltage output of $10 \mathrm{~V}$ and a current of $0.6 \mu \mathrm{A}$ were recorded. This device design was optimized by $\mathrm{Hu}$ et al. to increase the output under similar loading conditions to $20 \mathrm{~V}$. [52]

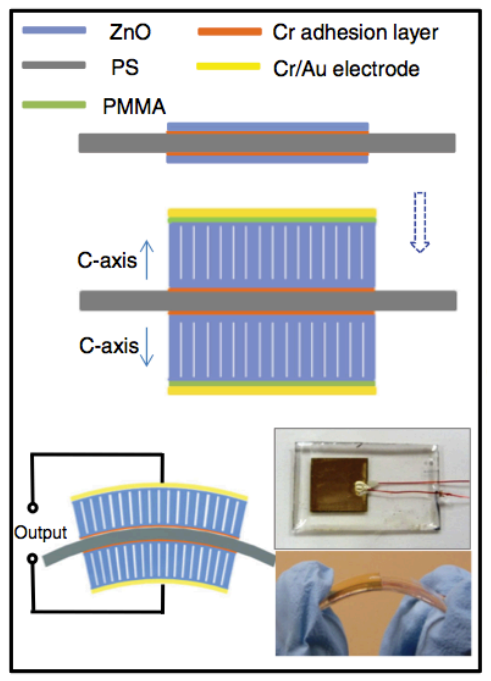

Fig. 3.6 - Fabrication of vertically-aligned nanowire generator based on a polystyrene substrate [50]. 
It was found that plasma treating of $\mathrm{ZnO}$ before PMMA encapsulation was an affective method for increasing electrical output, but this increase was only observed for a short time (two weeks) after device assembly. It was believed the hydrogen atoms may reabsorb on the $\mathrm{ZnO}$ surface or the plasma-induced oxygen vacancy injection my effuse during this time. The need for a better environmental shielding and packaging technology was highlighted. Hu et al. also state that after loading the harvester for 1000 strain cycles, the power output was high enough to run a commercial electronic watch for more than one minute [50].

Initial fabrication in Phase I was concerned with optimizing the deposition and growth of the functional $\mathrm{ZnO}$ nanowires. An understanding of the growth parameters associated with low-temperature deposition processes was needed to successfully control nanowire length, diameter, density, and tip geometry. These nanowire characteristics were successfully controlled by manipulating growth solution chemical composition, growth time, growth temperature, and seeding condition for crystal growth.

Phase II focused on transitioning the optimized rigid design to a flexible platform. The Phase III device designs further evolved the active harvesting device to a flexible system based on a Polydimethylsiloxane (PDMS) platform. Transferring from a PEN to PDMS platform introduces a number of processing challenges due to the hydrophobic property of the PDMS surface, but this design shift was seen as a beneficial step towards in vitro and in vivo testing of piezoelectric devices [15,31]. 
A schematic of the three design phases is presented in Fig. 3.7.

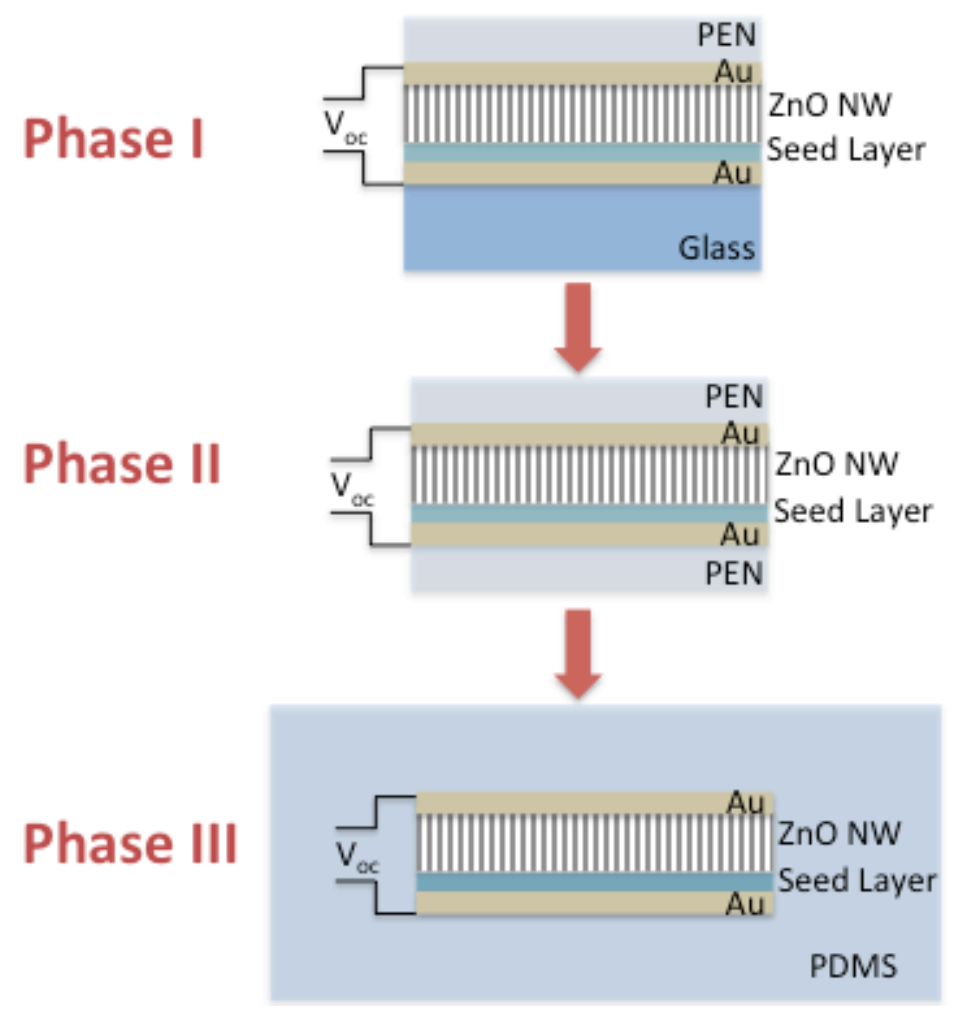

Fig. 3.7 - Schematic of nanogenerator design progression for Phases I-III.

\subsection{Experimental Methods}

\subsubsection{Phase I and II Development}

A bottom-up development approach was implemented in order to develop an initial harvesting/sensing device design featuring a functional piezoelectric $\mathrm{ZnO}$ nanostructure array. The initial design was based upon a relatively simple 'composite' of a number of previously constructed nanogenerator in the literature. Development of a standard fabrication procedure, which yielded a measureable electrical response, was the ultimate goal of this initial work. Subsequent sub-sections describe the basis and rationale for selection of each device component. 


\subsubsection{Substrate Selection}

Although a completely flexible stack design is the aim of this work, fabrication began on a glass substrate. This was done to ensure subsequent fabrication steps, such as electrode deposition and $\mathrm{ZnO}$ growth could be successfully achieved without the added thermodynamic and mechanical challenges associated with deposition on flexible substrates. Borosilicate glass cover slides (25 mm x $25 \mathrm{~mm}$, Fischer Scientific) were used as the platform for all further fabrication of the initial devices.

\subsubsection{Electrode Deposition}

Prior to electrode deposition, glass substrates were cleaned using a standard procedure consisting of successive baths in acetone, isopropyl alcohol, and deionized water for five minutes each. Samples were blown dried using $\mathrm{N}_{2}$ gas before a dehydration bake at $150^{\circ} \mathrm{C}$ to remove any absorbed surface moisture.

Metal electrodes were sputtered onto the clean glass surface using a CVC 610 DC Magnetron Sputtering Station. Au was selected for the electrode material due to its high conductivity, ductility, and use in a majority of device designs of its kind. Prior to deposition of the gold film, an intermediate Ti layer (5-10 nm) was deposited using DC sputtering to account for the lattice mismatch between the glass and $\mathrm{Au}$ film, increasing film adhesion. Directly after titanium deposition, $\sim 500 \mathrm{~nm}$ of gold was deposited to act as the bottom electrical contact. After deposition, an adhesive tape test was conducted to ensure full film adhesion. 
A number of different thicknesses of gold films were deposited to optimize the balance between electrical conductivity and film integrity. If a gold film was relatively thick $(>1$ $\mu \mathrm{m})$, microcracking was observed due to residual film stresses. The eventual goal of optical transparency was considered, but initially ignored in the interest of further fabrication steps.

Rigid-substrate designs typically include a gold annealing processing step after deposition $[86,118]$. Heating the sample to $300-350^{\circ} \mathrm{C}$ achieves a more uniform and atomically flat $\mathrm{Au}(111)$ surface for further $\mathrm{ZnO}$ growth. The atomic scale of $\mathrm{ZnO}$ nucleation and growth results in a process that depends heavily on substrate roughness for nanowire orientation. Although this annealing step is important for the ultimate vertical alignment of grown nanowire, it was omitted due to future thermal limitations, as the fabrication shifts to polymeric substrates.

\subsubsection{ZnO Seeding}

Prior to nanowire growth, a seed layer $(\sim 20 \mathrm{~nm})$ of the nutrient solution was spin coated onto the patterned surface. The $\mathrm{ZnO}$ seeding procedure was based on work by Wang et $a l$, which used a $10 \mathrm{mM}$ solution of zinc acetate dihydrate in ethanol as the seeding solution [117]. Deposition was performed via spin coating onto the bottom gold electrode at $1000 \mathrm{rpm}$ for 60 seconds. The sample was then heated to $150^{\circ} \mathrm{C}$ for 10 minutes to remove the ethanol solvent, leaving $\mathrm{ZnO}$ crystals. The deposition steps were repeated three times to increase the number and size of seeding crystals. 
Other works used reactive RF sputtering to deposit 20 to $1000 \mathrm{~nm}$ of $\mathrm{ZnO}$ seed layers prior to the growth of nanostructures, but a solution-based seeding process was selected for this project to limit the requirement for high-vacuum processing as much as possible [118] Ji et al. found that thinner seed layers with poor crystal characteristics lead to poorly aligned nanowires, while nanostructures grown on thicker seed layers with good crystalline structures yielded better vertical alignment. It was also found that by increasing seed layer thickness, nanowires did not coalesce, increasing the nanowire diameter.

\subsubsection{ZnO Nanowire Growth and Characterization}

Growth of the $\mathrm{ZnO}$ nanostructures was carried out using the aqueous chemical assisted process developed by Vayssieres. [72]. A 1:1 solution of zinc nitrate and hexamethylenetetramine (HMTA) was used as the nutrient growth solution. Samples were floated on the surface of the nutrient solution to prevent any precipitates from depositing on the surface during growth. The effect of processing parameters including nutrient solution concentration, growth time, and solution temperature were varied to obtain $\mathrm{ZnO}$ nanowires of various densities and geometries. A concentration range of the precursor solution between $0.1-5 \mathrm{mM}$ controlled the density of $\mathrm{ZnO}$ nanowires, while varying the growth time between 6-48 hours and processing temperature between $60-$ $80^{\circ} \mathrm{C}$ affected the nanostructure's length and diameter. Table 1 presents the growth parameters used to develop the optimal growth process. Measured radius and nanowire density are also presented. 
Table 1 - Results of varying hydrothermal growth parameters and measured nanowire radius and average density.

\begin{tabular}{|c|c|c|c|c|c|c|}
\hline $\begin{array}{c}\text { Solution } \\
\text { Molarity }\end{array}$ & $\begin{array}{c}\text { Growth } \\
\text { Time (hrs) }\end{array}$ & $\begin{array}{c}\text { Growth Temperature } \\
\text { (deg C) }\end{array}$ & Substrate & $\begin{array}{c}\text { Seed Layer Drying } \\
\text { Temperature (deg C) }\end{array}$ & $\begin{array}{c}\text { Average Radius } \\
\text { (nm) }\end{array}$ & $\begin{array}{c}\text { Density (\#/100 } \\
\text { um2) }\end{array}$ \\
\hline 0.025 & 24 & 95 & Glass & 100 & 25 & 2400 \\
\hline 0.01 & 24 & 95 & Glass & 100 & 24 & 1100 \\
\hline 0.025 & 24 & 70 & Glass & 100 & 54 & 1800 \\
\hline 0.025 & 24 & 70 & Glass & 150 & 86 & 1600 \\
\hline 0.025 & 48 & 70 & Glass & 100 & 98 & 1400 \\
\hline 0.025 & 24 & 95 & Au/Glass & 100 & 36 & 750 \\
\hline 0.025 & 24 & 70 & PEN & 100 & 45 & 1000 \\
\hline
\end{tabular}

The morphology of the nanowires was observed using an SEM with a field emission gun and equipped with an EDX detector. When observing the semiconducting $\mathrm{ZnO}$ nanostructures, a $10 \mathrm{kV}$ accelerating voltage yielded the most adequate images while minimizing charging effects. When observing insulating samples such as polymeric layers, $15 \mathrm{~nm}$ of Au was sputtered on the surface and an accelerating voltage of $5-10 \mathrm{kV}$ was used to reduce surface charging.

\subsubsection{Growth Condition Optimization}

Initially grown on glass substrates that were previously seeded with $\mathrm{ZnO}$ crystals, the optimum growth concentration was found to be $0.025 \mathrm{M}$. The solution concentration determined the density of nanowires on the growth surface (reported as number of nanostructures per $100 \mu \mathrm{m}^{2}$. Analysis of density and average nanowire diameter was performed using image analysis software. It was observed that lower molarity solutions yielded lower densities. Under the same growth time and temperature, but increasing the solution molarity from $0.01 \mathrm{M}$ to $0.025 \mathrm{M}$, nanowire density doubled from 1100 to 2400 per $100 \mu \mathrm{m}^{2}$. The higher concentration of $\mathrm{Zn}$ in the seed solution creates more nucleation 
cites, and therefore a higher density of $\mathrm{ZnO}$ nanowires. [60,75]. As the solution molarity which is further increased to $0.05 \mathrm{mM}$, resulting nanowires exhibited both poor morphology and alignment attributed to their high nucleation and coalescence of nanowires early in the growth. Figure 3.8 shows the $\mathrm{ZnO}$ nanowires arrays exhibiting varying densities due to the selected growth conditions.

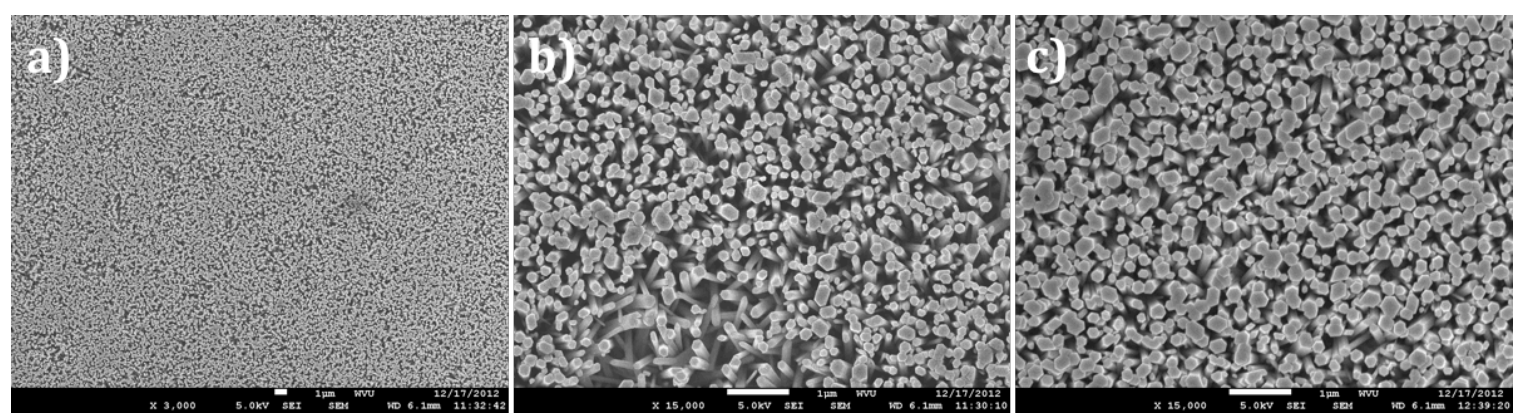

Fig. 3.8 - SEM micrographs of hydrothermally grown nanowires with a growth solution concentration of $0.025 \mathrm{M}$ and a growth temperature of $70^{\circ} \mathrm{C}$ with a $150^{\circ} \mathrm{C}$ seed layer annealing and a growth time of a-b) 24 hours c) 48 hours.

While the concentration of the zinc nutrient growth solution controls the density, the growth time and temperature govern the length and diameter of the resulting nanowires. A growth time of 24 hours resulted in nanowires with a length $\sim 2 \mu \mathrm{m}$. This was determined to be an optimum length for further device development. Fig. 3.9 presents SEM micrographs of nanowires grown using various growing conditions. 

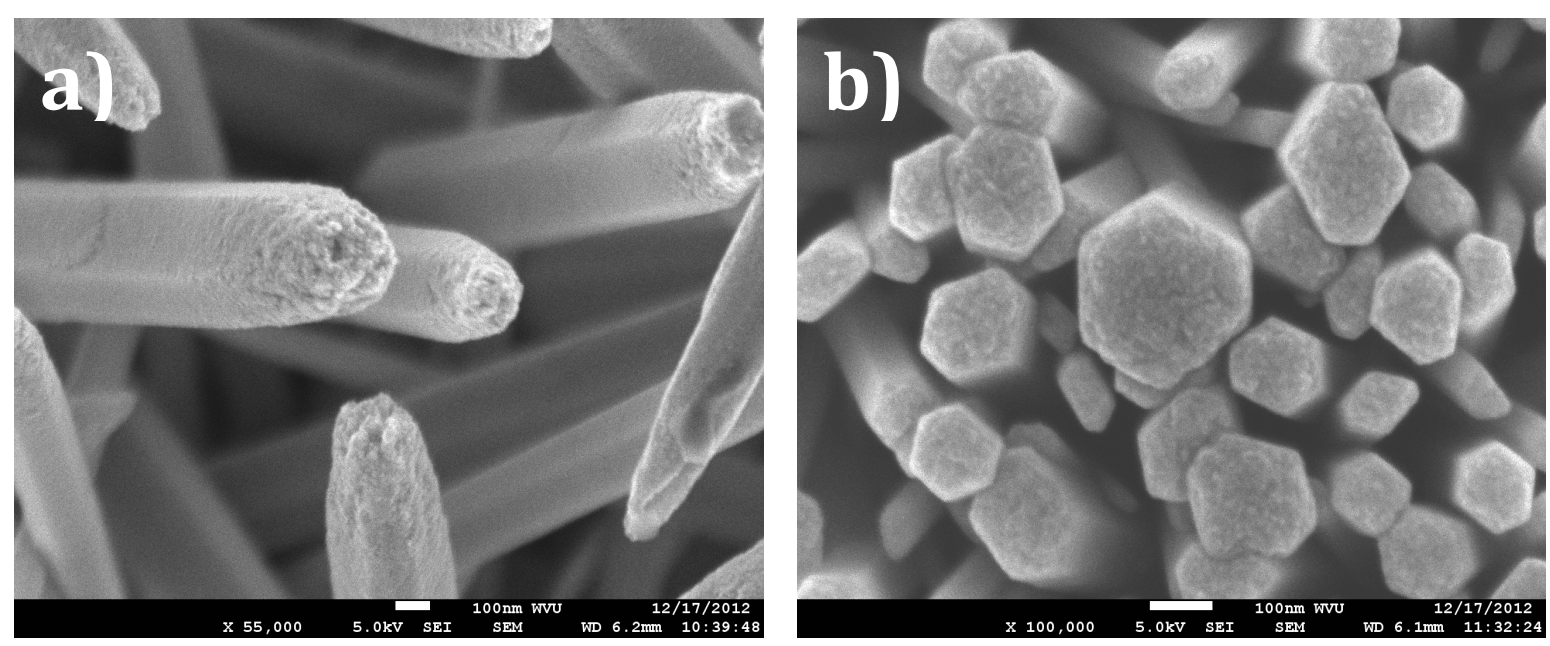

Fig. 3.9 - SEM micrographs of the tip geometry under a) $0.01 \mathrm{M}$ concentration, $95^{\circ} \mathrm{C}$, and 24 hours of growth time, b) $0.025 \mathrm{M}$ concentration, $70^{\circ} \mathrm{C}$, and 48 hours of growth time.

Microstructural studies were performed on $\mathrm{ZnO}$ nanostructures using a Bruker D8

Discover diffractometer. X-Ray diffraction (XRD) provided results regarding the orientation and intensity of specific crystal structures. XRD analysis focused primarily on the $\mathrm{ZnO}(002)$ peak, which occurs at $34.5^{\circ}$ and the piezoelectric c-axis orientation. XRD results for various samples of hydrothermally grown $\mathrm{ZnO}$ nanowires are presented in Fig. 3.10 . 

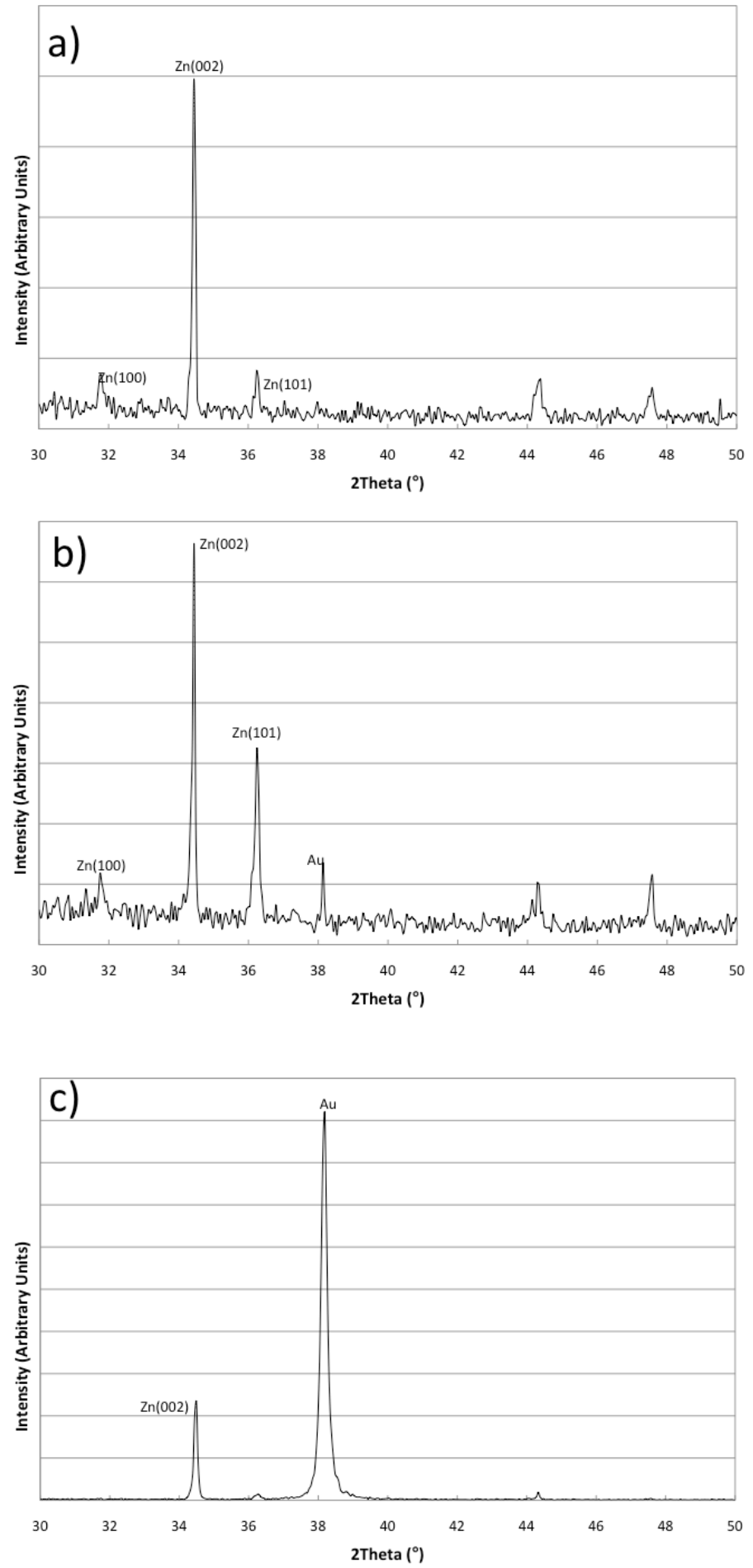

Fig. 3.10 - X-ray diffraction of grown $\mathrm{ZnO}$ nanowires on a) $\mathrm{ZnO}$ seed layer on glass b) on gold-coated glass c) on gold coated PEN. 
The XRD analysis of the three samples in Fig. 3.10 all show diffraction spectra with prominent $\mathrm{ZnO}(002)$ peaks. SEM analysis presented in Fig. 3.11 confirmed the poor vertical alignment.

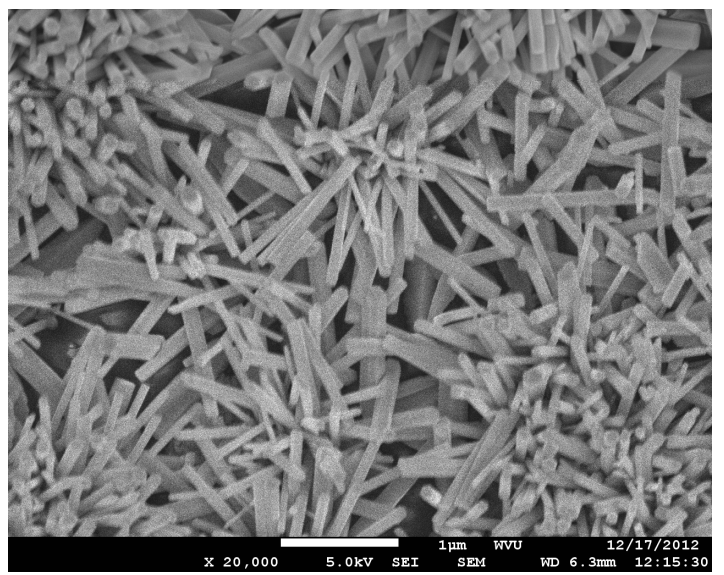

Fig. 3.11 - SEM image of hydrothermally grown nanowires on a gold-coated PEN substrate.

\subsubsection{Fabrication of $\mathrm{ZnO}$ Arrays}

Patterning of the $\mathrm{ZnO}$ arrays was considered to allow for more options during the final design. Traditional photolithography was performed to fabricate alternating parallel lines of $\mathrm{ZnO}$ nanowires and spaces to be both filled by a dielectric matrix.

AZ 3330-F (AZ Electronic Materials) was used as the primary positive photoresist for all fabrication work. AZ 3330-F was chosen because of its ease of processing, relatively low cost, and thermal stability up to $125^{\circ} \mathrm{C}$.

Prior to the $\mathrm{ZnO}$ seeding step, the photolithography process yields patterns on the surface of the bottom electrodes. Substrates were initially cleaned as previously described. To avoid film adhesion issues, hexamethyldisilazane (HMDS) was used as an adhesion 
promoter between the Au surface and photoresist. A thin film of HMDS was spin coated on clean substrates at $7500 \mathrm{rpm}$ for 30 seconds at an acceleration of $3750 \mathrm{rpm} / \mathrm{s}$. AZ $3330-\mathrm{F}$ was then spin coated at $5000 \mathrm{rpm}$ for 60 seconds at an acceleration of $2500 \mathrm{rpm}$ per second, yielding a photoresist thickness of $\sim 2.5 \mu \mathrm{m}$.

After coating of the photoresist, samples were soft-baked on a hotplate for 60 seconds at $90^{\circ} \mathrm{C}$. This initial baking removes the remaining solvent and renders the photoresist photoactive. A transparent Mylar mask was used in conjunction with a UV source to produce the desired pattern in the previously deposited photoresist layer. An OAI UV Flood Exposure system provided the sample with proper dose of UV light $(\lambda=365 \mathrm{~nm})$ to render the exposed regions soluble in the developer solution.

Once the samples were exposed, an AZ 300 MIF developer solution was used to remove the regions of the film exposed to UV light. Samples were submerged in developer solution and gently agitated by hand for 45 seconds. To stop the development process, the samples were submerged in deionized water and spray-dried with $\mathrm{N}_{2}$ gas.

The final step of the positive photolithography process was a hard-bake of the remaining patterned photoresist on a $90^{\circ} \mathrm{C}$ hotplate for 60 seconds. This final bake solidified the photoresist for further processing.

Once patterning was complete, the samples were seeded with $\mathrm{ZnO}$ crystals and $\mathrm{ZnO}$ nanowires were grown as previously described. Next, the patterned photoresist was 
stripped in an acetone bath prior to deposition of the PMMA dielectric matrix.

Subsequent device fabrication steps were performed as described for non-patterned samples. SEM micrographs of patterned parallel line $\mathrm{ZnO}$ nanowire arrays is shown in Fig. 3.12.

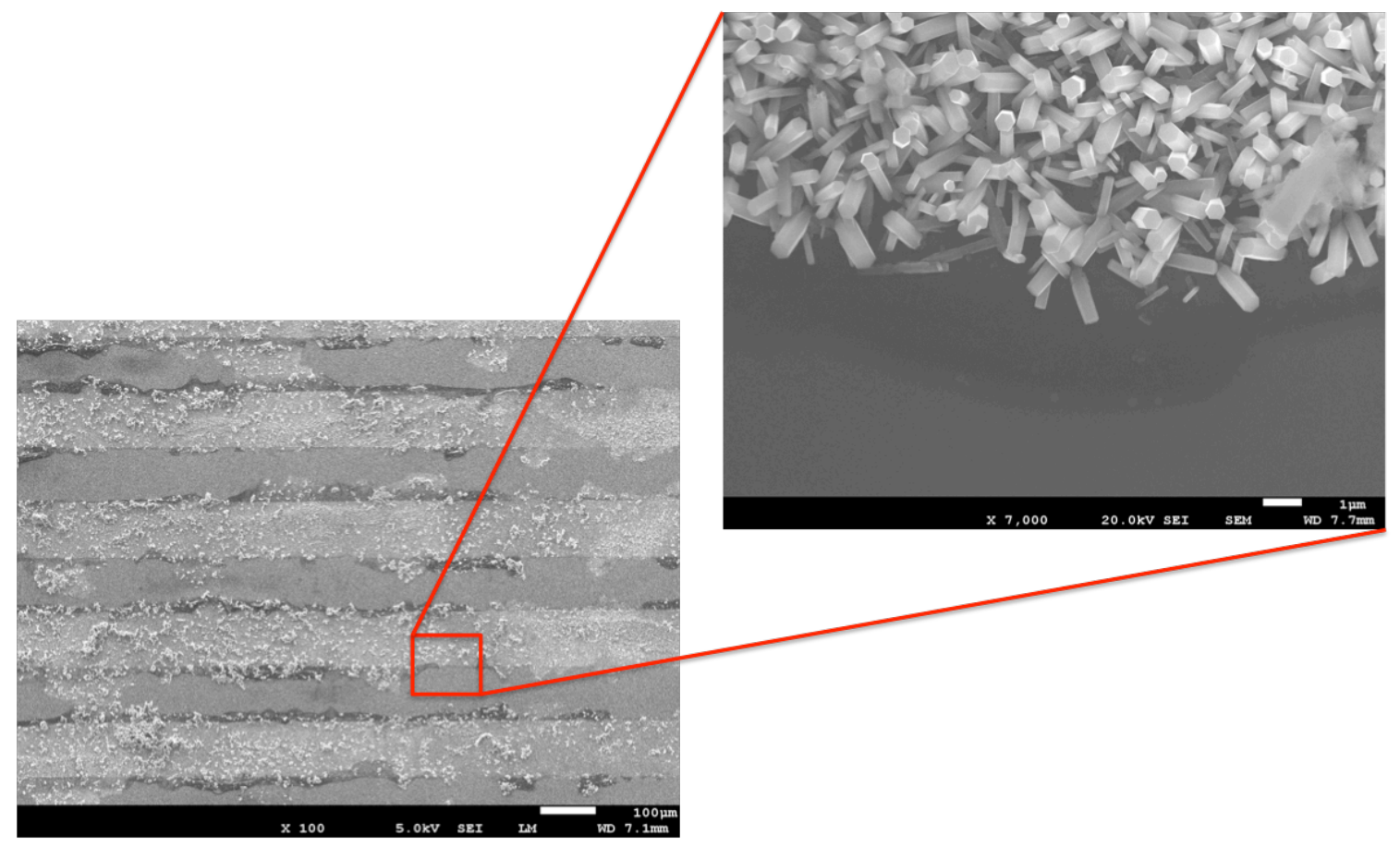

Fig. 3.12 - SEM micrograph of patterned $\mathrm{ZnO}$ nanowire arrays using photolithography techniques.

\subsubsection{Dielectric Matrix}

After growth of $\mathrm{ZnO}$ nanowires on the gold-coated substrates, a dielectric material was deposited to encase the nanowires and prevent electrical shorting across the top and bottom electrodes. Previous investigations have used Poly(methyl methacrylate) (PMMA) as the dielectric matrix surrounding the nanowires [49]. Although in most cases relatively thin $(0.2-3 \mu \mathrm{m})$, the PMMA matrix adds to the mechanical robustness of the 
functional layer and helps to support the piezoelectric nanowires' response while under various applied loads.

Based on the design of similar nanogenerators, PMMA was deposited and cured on the exposed $\mathrm{ZnO}$ array. Three layers of PMMA were spin coated at $500 \mathrm{rpm}$ for 30 seconds each with a hot-plate baking at $150^{\circ} \mathrm{C}$ for 60 seconds between each deposition spin. The total thickness of the PMMA layer was $\sim 2.5 \mu \mathrm{m}$, encapsulating the $2 \mu \mathrm{m}$ long nanowires completely.

Once the dielectric matrix was deposited and cured, samples were uniformly etched using a March PX-250 Plasma Asher. Oxygen plasma treating at 15W for 3 minutes served two functions. First, the $\mathrm{O}_{2}$ ions etch the PMMA surface, thus exposing the tips of the $\mathrm{ZnO}$ nanowires. Secondly; the plasma cleans the surface and increases adhesion of the top electrode.

The need to expose the nanowire tips to form an intimate contact with the top electrode is somewhat disputed in the literature. Work by Wang et al. presents a nanogenerator design that cites the requirement of the nanowire tips to make intimate contact with the top electrode to form a Schottky barrier contact [121]. Later works cite an increased output associated with leaving a thin layer $(<1 \mu \mathrm{m})$ of PMMA between the nanowire tips and the top electrode [119-121].

\subsubsection{Top Electrode Fabrication}


The top electrode was designed to be flexible to maximize the applied contact force that is transferred, to increase the strain on the $\mathrm{ZnO}$ nanowires, therefore increasing the electrical response. Commercially available Teonex ${ }^{\circledR}$ PEN films (DuPont Teijin) were used as the base for the top electrode. PEN was chosen due to its high transparency, low surface roughness, high heat resistance $\left(\mathrm{T}_{\mathrm{g}}=120^{\circ} \mathrm{C}\right)$, and excellent solvent resistance. PEN films used also featured a planarized surface for increased adhesion.

Gold films with an intermediate Ti layer were deposited on PEN samples (25 mm x 25 $\mathrm{mm}$ ) following the same procedure previously describe for $\mathrm{Ti} / \mathrm{Au}$ deposition on glass. One change made to the standard substrate cleaning process to adapt to the thermal limit of the PEN substrate was a reduction in the absorbed moisture bake to $110^{\circ} \mathrm{C}$ from $150^{\circ} \mathrm{C}$. A reduction in film adhesion of the metal electrode with the PEN surface was not observed after this change was made. Sputtering times for Ti and Au were consistent with the deposition on $\mathrm{Au}$.

\subsubsection{Device Assembly}

To assemble the device, the top electrode on the PEN substrate was sandwiched on top of the previously fabricated device base. Adhesive tape was used to fasten the top and bottom platforms into one device. Care was taken while applying the tape to not cause pre-stresses in regions of the device and to leave exposed areas for electrical contact on the top and bottom electrodes. Wire leads (30 AWG, Radioshack) were attached using a small bead of graphite-filled conductive wire glue. A summary of the complete fabrication process is shown in Fig. 3.13. 

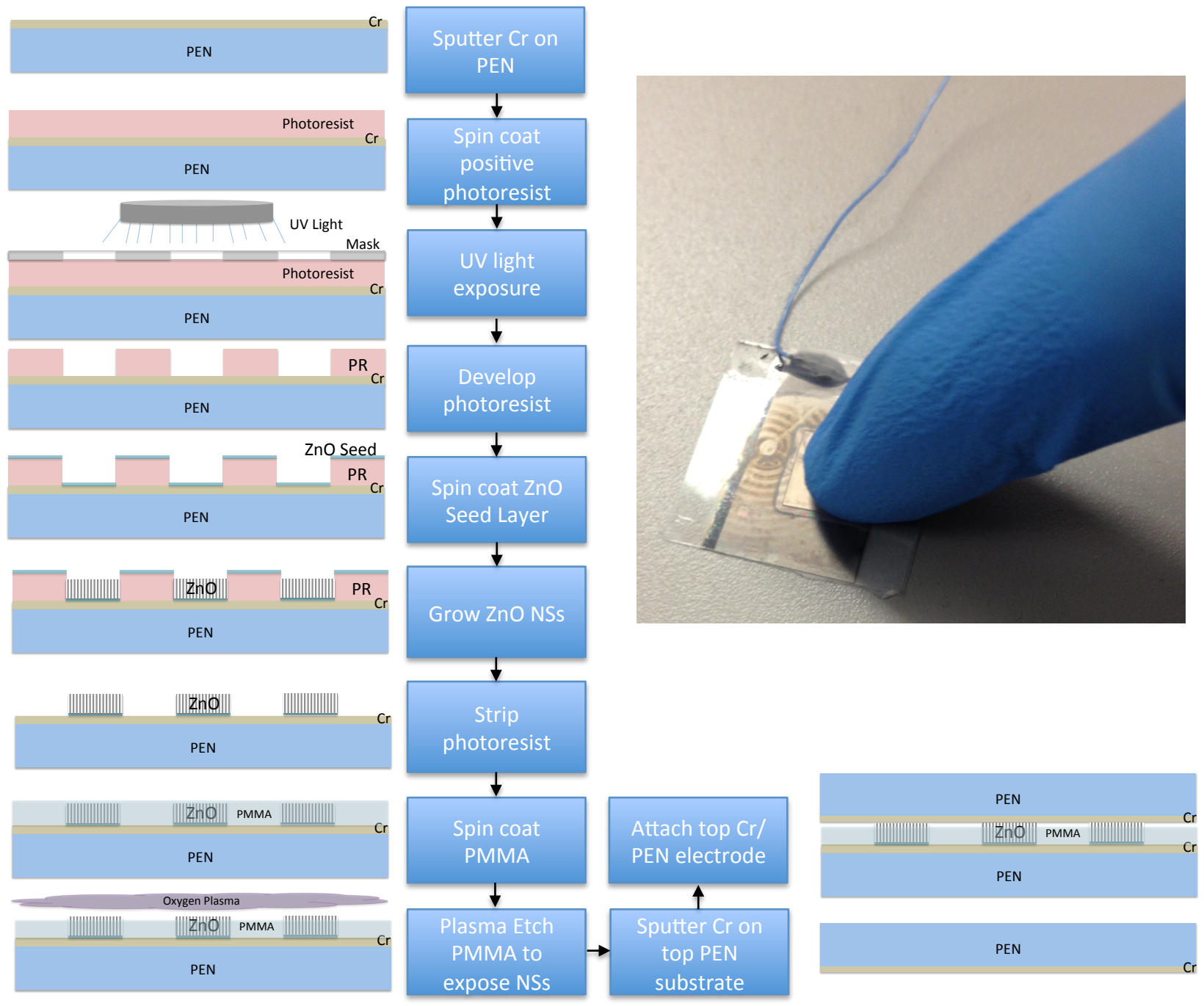

Fig. 3.13 - Fabrication process of the Phase II, flexible PEN-based sensor design with image of a fabricated device under fingertip loading.

\subsection{Results and Discussion}

\subsubsection{Electro-mechanical Device Characterization}

With a lack of a standardized testing procedure for piezoelectric sensors and nanogenerators, testing parameters were developed to attempt to quantify the failure associated with reduced electrical output after large mechanical loads and repeated fatigue loading scenarios. 
Once fabrication of the model devices was completed, testing of the electrical response under various loading scenarios was conducted. While a number of previous works measure open-circuit voltage of the device under the applied load of a finger or a hand, a more standardized and repeatable loading scheme is required to investigate the long-term functionality, especially in the case of long-term use.

A Leco M-400-G Microindentation hardness tester was modified and used to apply a uniform contact load to fabricated devices. Equipped with a Vicker's hardness indenter tip, devices were loaded to a maximum load between $10 \mathrm{~g}$ and $1 \mathrm{~kg}$ with a constant loading and unloading rate. A $25 \mathrm{~mm}^{2}$ stainless steel plate was placed between the indenter tip and the device surface to distribute the contact force. This loading is believed to more closely mimic contact area and pressures from an implement such as a finger or a stylus as apposed to a single point-loading scenario with high contact pressures (Fig. $3.14)$.

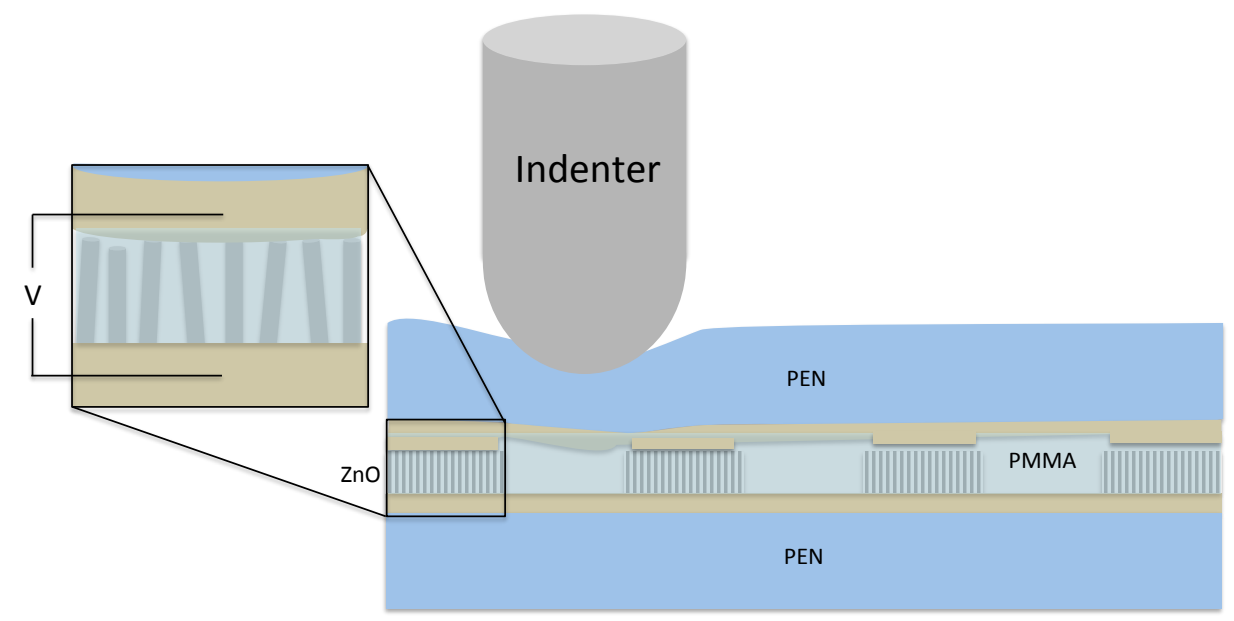

Fig. 3.14 - Diagram of repeated micro-indentation testing of Phase II devices. 
During loading and unloading of the device, the in situ open-circuit voltage was recorded. An Agilent 34970A Data Logger Switch Unit featuring an internal 6 1/2-digit digital multimeter and 40 addressable channels was used for all electrical measurements. Care was taken during measurements to ensure the measured output was truly an active output and not an artifact of the measurement system.

Preliminary results from model device performance and reliability analysis is presented below. In situ and ex situ failure analysis was performed on PEN-based device designs. Fig. 3.15 shows the open-circuit voltage response observed under tactile loading using a nitrile-gloved fingertip. The device was loaded and unloaded several times, to ensure a measureable response was present and successful device fabrication was achieved. 

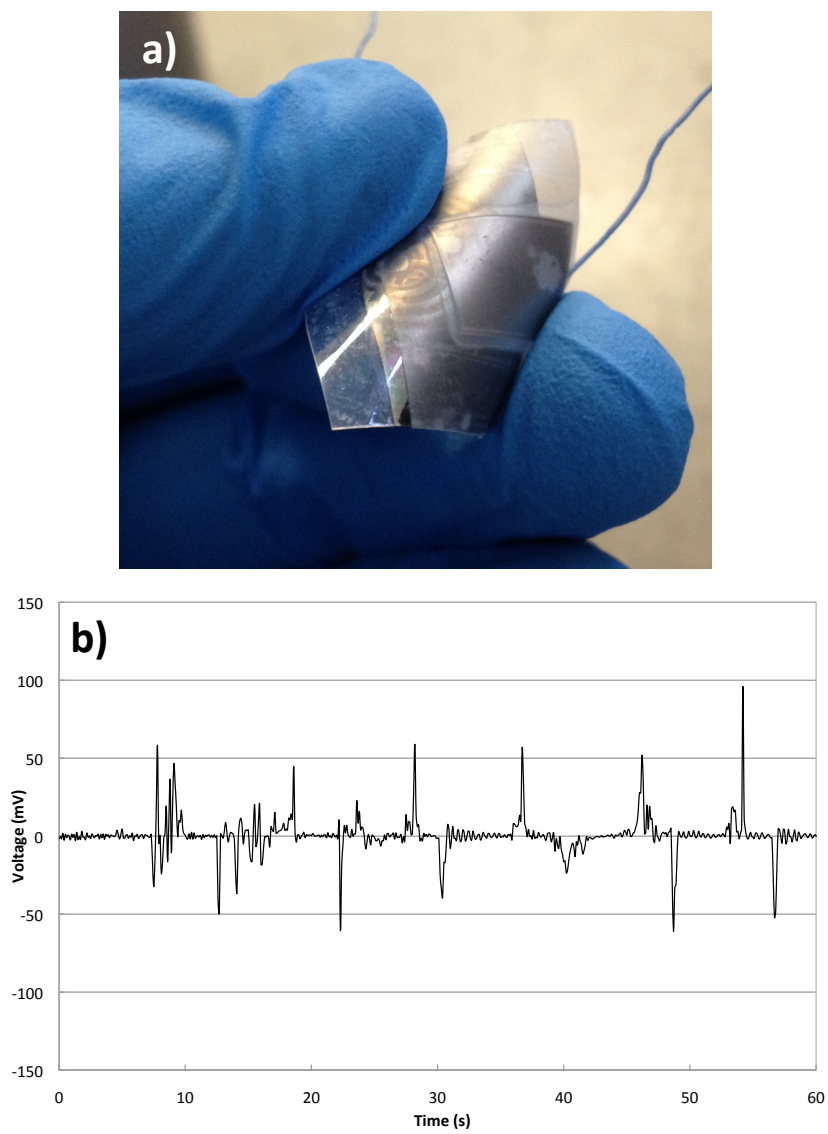

Fig. 3.15 - a) Fingertip buckling loading of flexible Phase II device design b) Open-circuit voltage measured under buckling.

A maximum peak voltage response of nearly $100 \mathrm{mV}$ was observed. The shapes of the voltage curves were consistent with the piezoelectric potential created in the $\mathrm{ZnO}$ nanowires as they are stressed and relaxed. Though some signal noise was present and believed to be from the electrode connections, a positive jump in voltage was observed when the load was applied to the device. Once the load was released, the transient flow of electrons travel in the reverse direction, causing a complementary voltage spike in the opposite direction. This was observed for all samples. 
After the initial testing of mechanical deformation from fingertip loading, the microindentation platform provided a more standardized means of loading. With the stainless steel counterface on the device service to distribute the contact load, various contact pressures (ranging from 20-392 $\mathrm{kPa}$ ) were applied to the PEN-based device surfaces. Representative voltage responses are presented in Fig. 3.16.

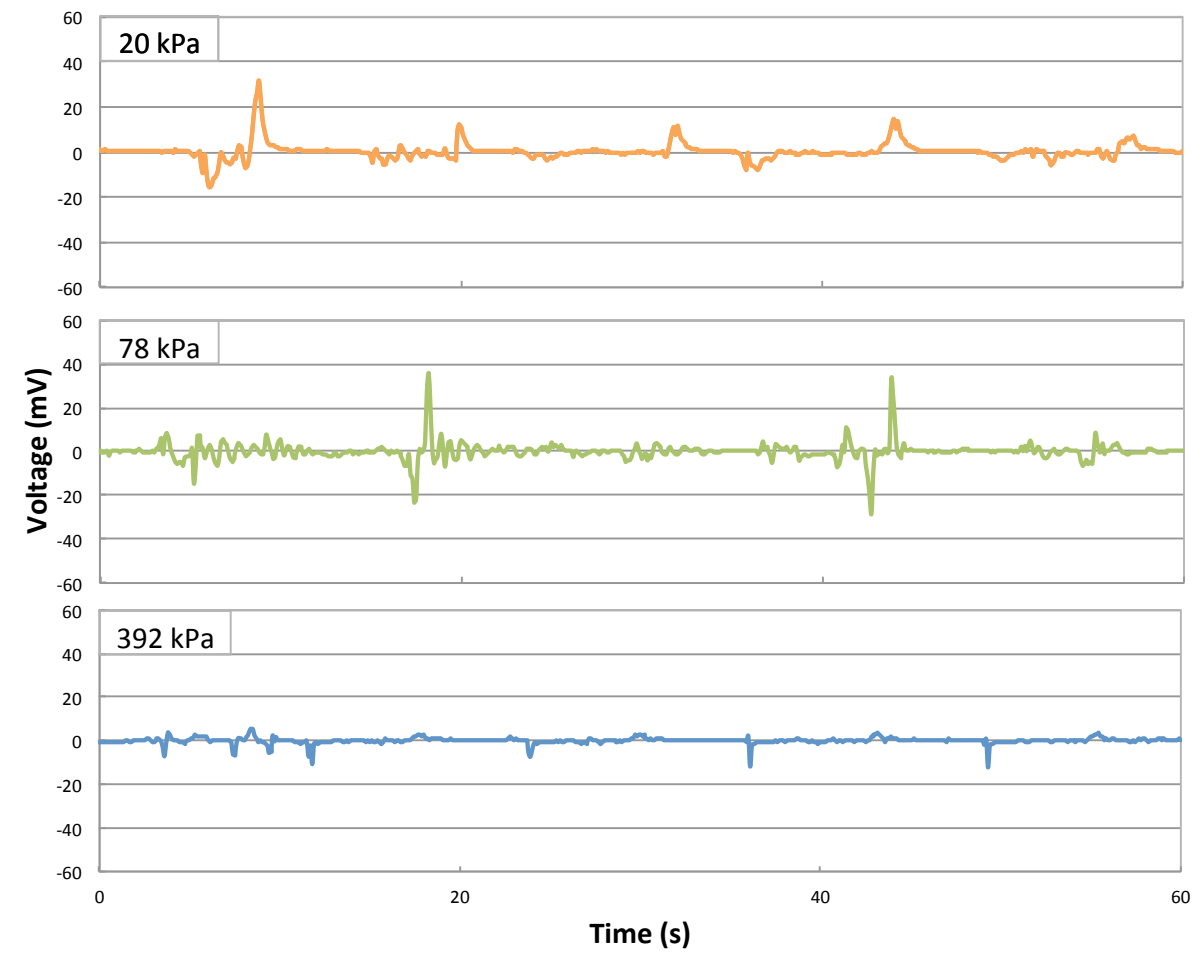

Fig. 3.16 - Electrical voltage output of PEN-based device under various applied contact pressures using the microindentation testing scheme.

Voltage response curves showed the same loading and relaxing peaks as previously observed. At a low contact pressures between $20-78 \mathrm{kPa}$, peak voltages of $\sim 31-33 \mathrm{mV}$ was observed. As contact pressure increased to $196 \mathrm{kPa}$ and above, a reduced voltage output was observed. Fig. 3.17 shows the superposition of peak voltage response curves of a PEN device under various applied contact loads. 


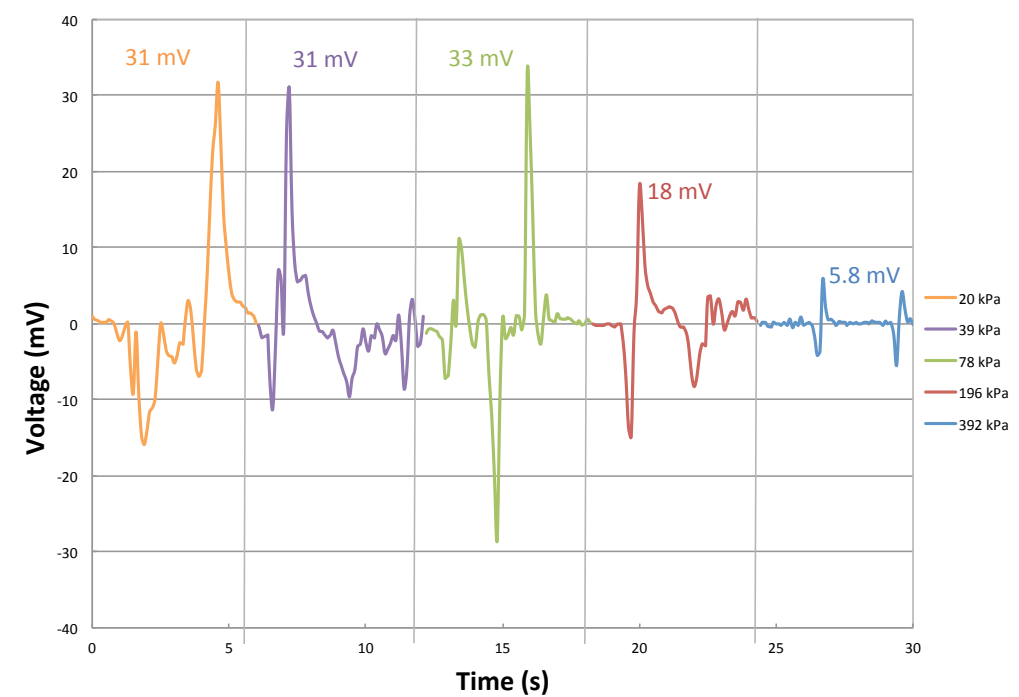

Fig. 3.17 - Superposition of peak electrical voltage output curves under each of the applied contact pressures.

The voltage response observed under a contact pressure of $392 \mathrm{kPa}$ was reduced to only $5.8 \mathrm{mV}$. This large drop in voltage response was attributed to failure of device components because the device performance would not return to its original output voltage, even as lower loads were used. Fracture of the nanowires and cracking of the electrodes were hypothesized to be the dominant cause of decreased output.

To investigate the failure of these PEN-based devices, they were disassembled to allow component-level failure analysis to be performed. Nanowire arrays of the devices were observed using an SEM. Micrographs of a PEN devices which was loaded with a contact pressure of $392 \mathrm{kPa}$ are shown in Fig. 3.18. 


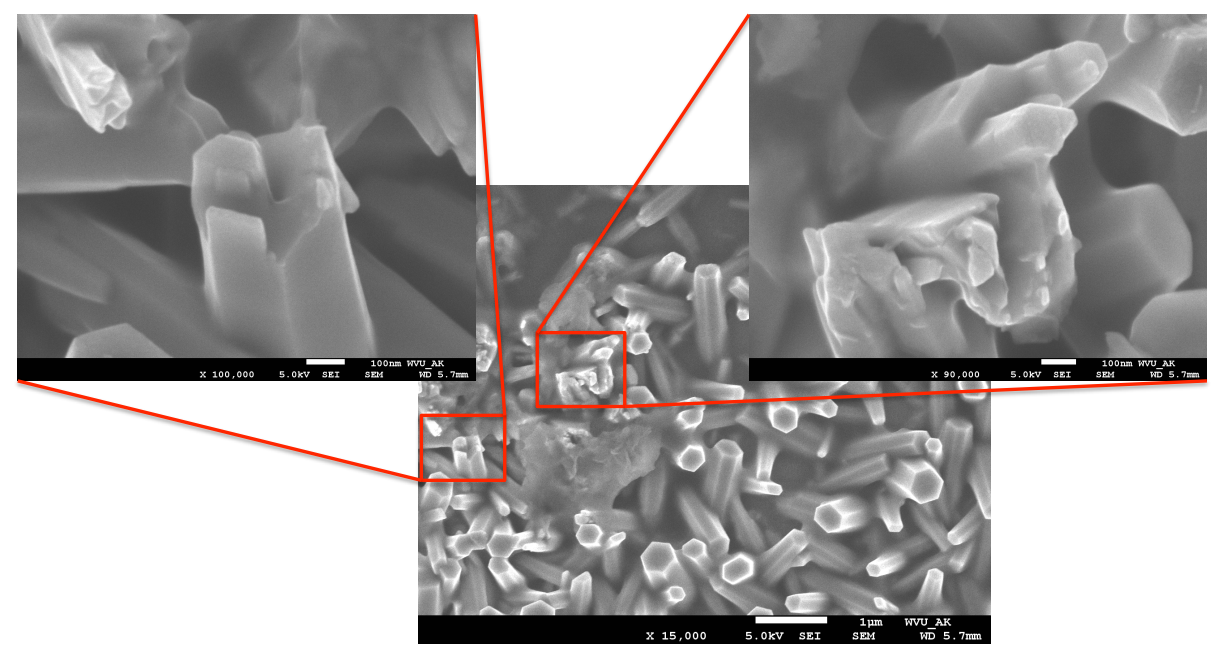

Fig. 3.18 - SEM failure analysis of $\mathrm{ZnO}$ nanowires after repeated loading at $392 \mathrm{kPa}$.

Analysis of the SEM micrographs shows evidence of nanowire fracture throughout the array. This is believed to significantly influence the reduction in voltage response previously observed. The top gold electrode counterface was also observed with the SEM for evidence of microcracks and compressive failure. Fig. 3.19 presents the observed contact-based failure of the electrode.

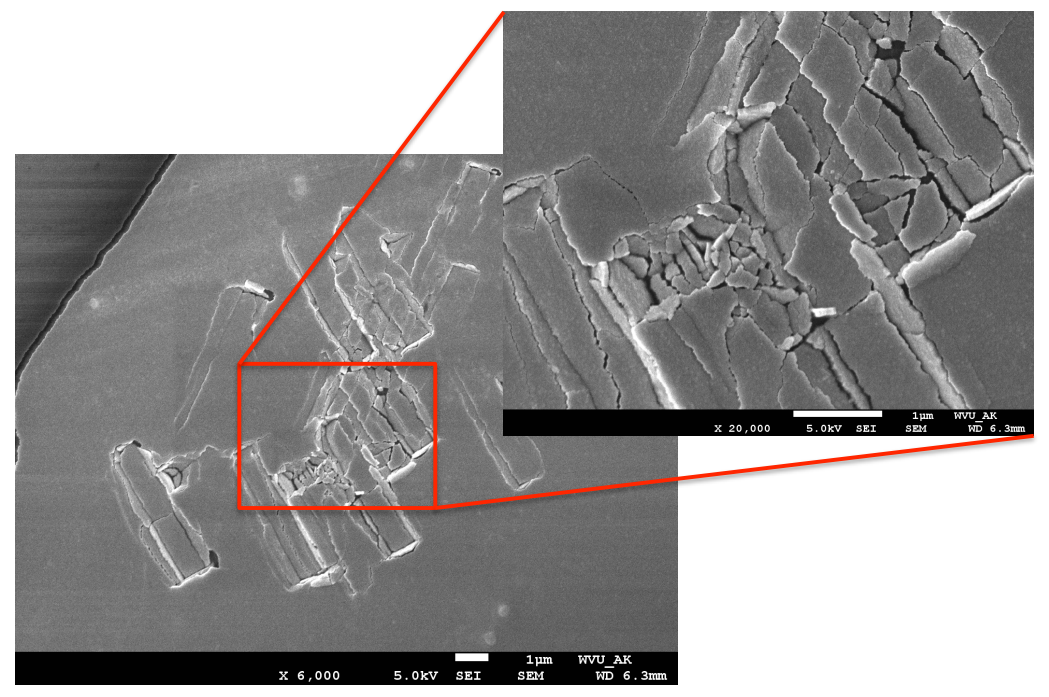

Fig. 3.19 - SEM failure analysis of top gold electrode after repeated loading at $392 \mathrm{kPa}$ of contact pressure. 
When observing deformed areas of the flexible PEN/Au electrode, microcracking was the dominant failure mechanism observed. As previously reported [92], damage of this conductive layer can lead to a compromise in device performance.

The presence of $\mathrm{ZnO}$ nanowire fracture and microcracking of the top gold electrode in the mechanically deformed PEN device shows the importance of further analysis of these device components. There is lack of investigations into this device system.

Nanoindentation was performed using a 10 micron sphero-conical tip to indent arrays of grown nanowires with varying densities. Measured hardness values were directly proportional to the nanowire density. When the nanowire density was increased from 11 nanowires per square micron to 24 nanowires per square micron, the indentation hardness increased $127 \%$. Figure 3.20 shows the load displacement curves for the performed indents. 


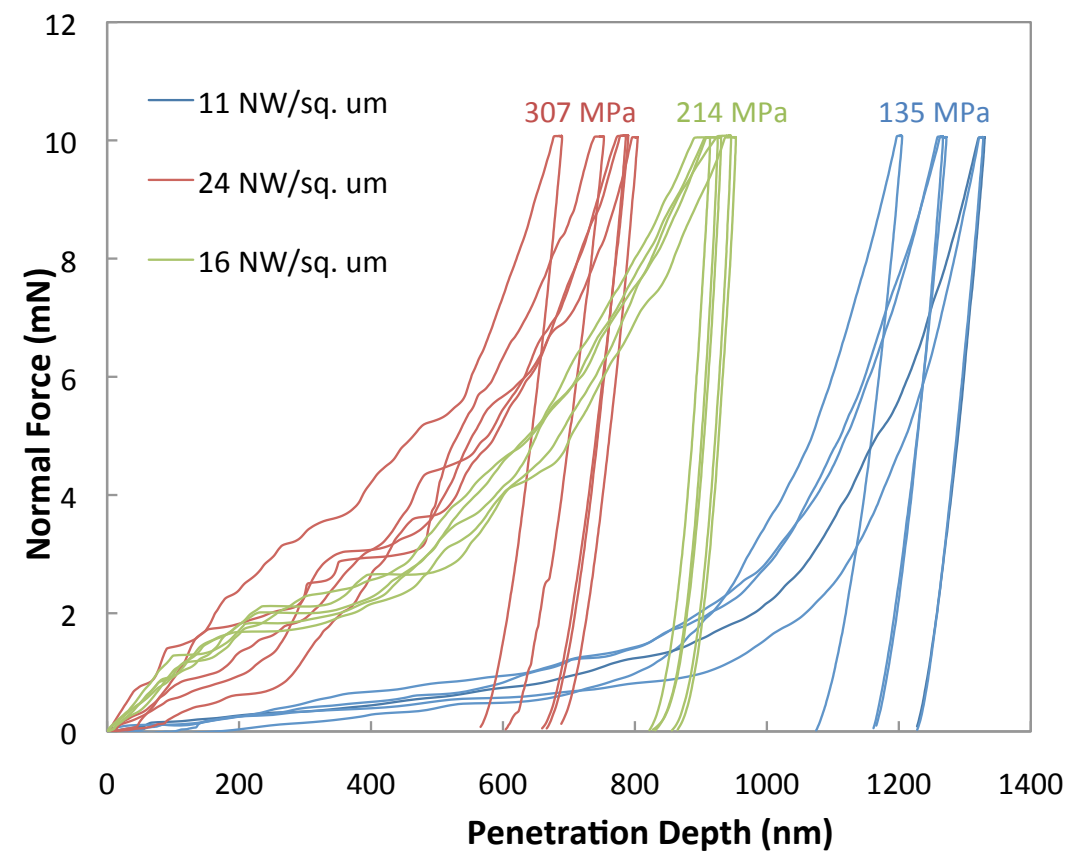

Fig. 3.20 - Load-displacement curves of 10 micron spherical tip nanoindentation on varied densities of $\mathrm{ZnO}$ nanowires and calculated hardness values.

\subsubsection{Phase III Development}

PDMS films were fabricated using Sylgard 184 (Dow Corning). This two-part silicone elastomer kit consists of a polymer base and a liquid curing agent, mixed at a ratio of 10:1 by weight. The base and curing agent were mixed using a magnetic stir bar at spin speeds below $200 \mathrm{rpm}$ to prevent the generation of excessive air bubbles. The solution was stirred for 1 hour to ensure thorough mixing of the components. After mixing, the polymer solution was placed in a vacuum dessicator to remove entrained air bubbles prior to film casting. A hand-pump dessicator was used at a pressure of $50 \mathrm{kPa}$ until a majority of visible air bubbles were removed (typically for around one hour).

For films requiring a thickness greater than $200 \mu \mathrm{m}$, traditional pour-casting techniques were used on glass substrates. Borosilicate glass substrates ( 2 in. $\mathrm{x} 2$ in.) were used as a carrier substrate. Prior to film casting, the glass was cleaned using the standard procedure 
previously described. Once the glass carrier substrates cooled to room temperature, a small volume $(\sim 3 \mathrm{ml})$ of un-cured PDMS solution was poured on the center of the substrate. Curing of the PDMS films was performed in an electric oven at $150^{\circ} \mathrm{C}$ for 10 minutes.

Thinner PDMS films $(<200 \mu \mathrm{m})$ were fabricated using a Laurel Technologies 400 spin coater. Cleaned glass carrier substrates were affixed on a vacuum chuck and rotated at speeds between 100-500 rpm. The thinnest films achieved using the 10:1 ratio polymer solution were $\sim 6 \mu \mathrm{m}$, deposited at $100 \mathrm{rpm}$.

\subsubsection{PDMS-based Electrode Fabrication}

The first challenge associated with the shift from a glass/PEN device design to a PDMS platform is that PDMS has an inherently hydrophobic surface. This makes metallization of PDMS difficult without additional pre-treating steps because of reduced adhesion forces between the coating and substrate. Plasma etching at $200 \mathrm{~W}$ for 30 seconds resulted in silanol groups on the surface. This creates a hydrophilic surface, allowing for greater adhesion during metallization. Ti/Au films were then deposited using the same process for glass and PEN substrates.

\subsubsection{Electrode Patterning}

To avoid adhesion and chemical compatibility issues with positive photoresist processing on PDMS substrates, a negative-tone photoresist was used for pattern formation on 
PDMS [122]. SU-8 2010 (MicroChem) was the negative photoresist used due to its ability to produce patterns with high aspect ratios and superior adhesion properties.

Previously cast PDMS films were not removed from the glass substrate carriers prior to photolithography processing. The PDMS/glass samples were prepared using the same cleaning process as previously described for PEN substrates. Spin coating was again used to deposit the resist. Because of the adhesion properties of the SU-8 resists, the adhesion promoter HMDS was not needed and was omitted from the process. SU-8 2010 was spin coated on the PDMS surface at $500 \mathrm{rpm}$ for 10 seconds at an acceleration of $100 \mathrm{rpm} / \mathrm{s}$ before increasing the speed to $3500 \mathrm{rpm}$ for 30 seconds with an acceleration of 300 $\mathrm{rpm} / \mathrm{s}$. The initial spin step allows the highly viscous resist to spread effectively before the faster spin speed thins the resist to a uniform thickness of $10 \mu \mathrm{m}$. After deposition of the SU-8 2010 film, the samples were baked on a hotplate at $95^{\circ} \mathrm{C}$ for 2.5 minutes.

A transparent Mylar mask was used, as previously described, with the exception that the negative-tone resist will yield a pattern opposite to that previously described positivetone resists process. This must be considered during photomask design, and therefore a dark-field mask is used. The transparent areas of the mask will allow the UV light to pass through and render the SU-8 resist insoluble in the organic developer solution. The flood exposure system was used to supply a $188 \mathrm{~mJ} / \mathrm{cm}^{2}$ dose of UV light.

A post-exposure bake was performed to further prevent the exposed regions from removal in the developer solution. This was performed on a hotplate at $95^{\circ} \mathrm{C} 3.5$ minutes. 
The samples were then developed in SU-8 developer solution for 2.5 minutes. Samples were immersed and gently agitated during the development process, before being washed in deionized water and dried using $\mathrm{N}_{2}$ gas.

After patterning, samples were plasma treated and Ti/Au films were deposited as before. The SU-8 pattern was then removed using a razor blade to split the layers on one corner peeling the two films apart.

\subsubsection{Hydrothermal Growth of ZnO Nanowires}

After successful deposition of the patterned $\mathrm{Ti} / \mathrm{Au}$ electrodes, $\mathrm{ZnO}$ nanowire growth was performed using the same procedure and processing conditions in Phase I and II. Growth of $\mathrm{ZnO}$ nanostructures on PDMS substrates was previously shown possible by Lin et al. [123].

\subsubsection{Device Construction}

Once the $\mathrm{ZnO}$ nanowires were grown the dielectric matrix PDMS was spin coated at $8000 \mathrm{rpm}$ for 30 seconds to achieve a film thickness of $6 \mu \mathrm{m}$. This thickness completely encased the $5 \mu \mathrm{m}$ long nanowires. The PDMS was then cured at $150^{\circ} \mathrm{C}$ for 5 minutes.

\subsubsection{Top Electrode Fabrication and Encapsulation}

To complete the device fabrication, the top Ti/Au electrode was sputtered as previously described, with the initial plasma treatment to ensure good adhesion between the Ti and PDMS. A final encapsulating layer of PDMS was pour cast on the device after 
attachment of electrical leads to the top and bottom electrodes to electrically isolate the top electrode and provide a layer of environmental protection. The encapsulating PDMS was cured at $150^{\circ} \mathrm{C}$ for 15 minutes. A figure summarizing the PDMS-based design process is presented in Fig. 3.21.
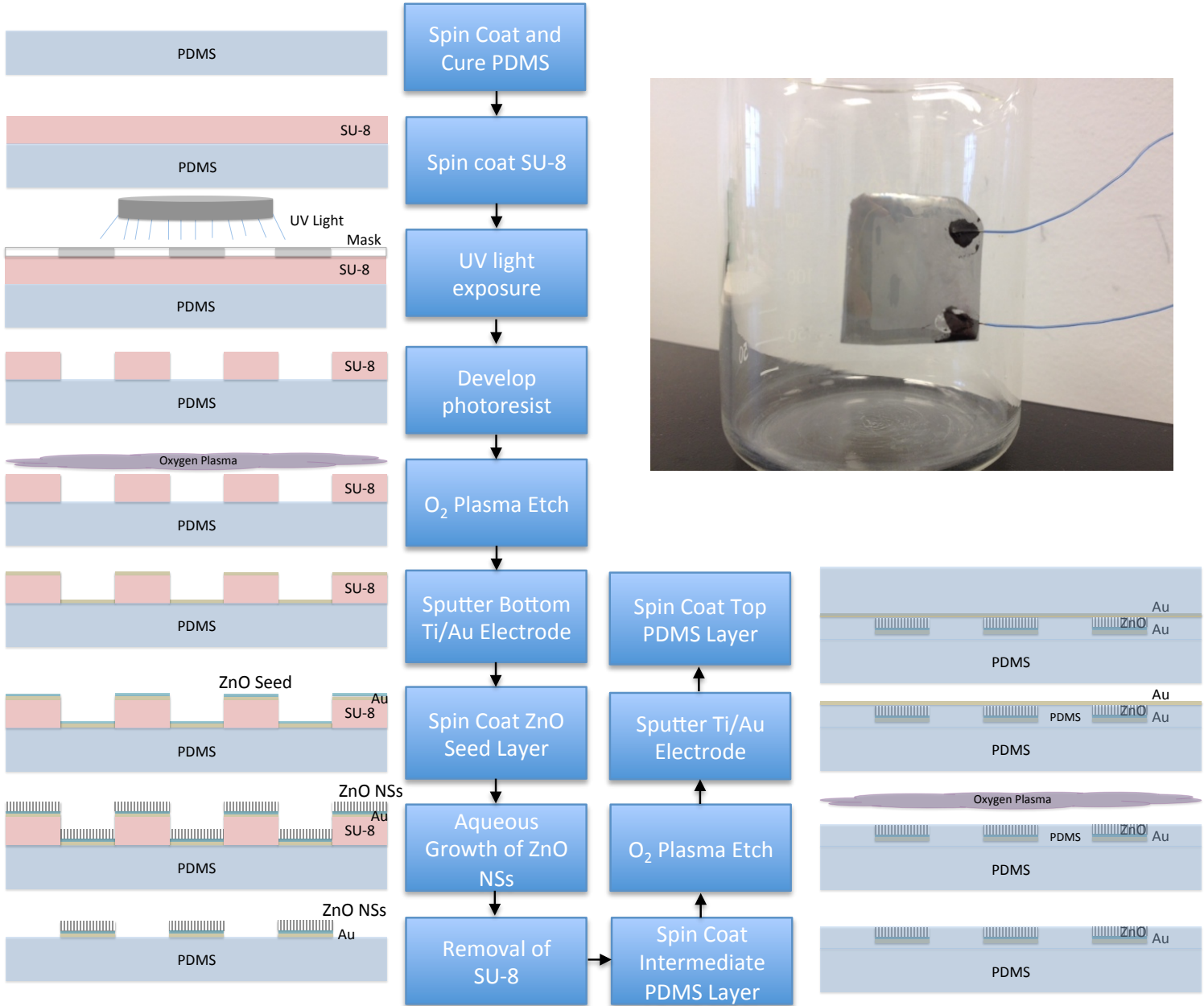

Fig. 3.21 - Schematic of fabrication process for the PDMS-based device design.

\subsubsection{PDMS-based Device Results}

Voltage response testing was performed as previously described using moderate fingertip loading to simply ensure successful fabrication. A voltage response of $\sim 8 \mathrm{mV}$ was 
measured during this loading. The significantly lower voltage output is believed to be due to the poor vertical alignment of the $\mathrm{ZnO}$ arrays as compared with the PEN-based device case.

With the increased flexibility and conformability of the PDMS platform, the fabricated PDMS device was loaded in tension by hand to show proof of tensile force response. Fig. 3.22 shows the voltage response measured when the PDMS was stretched.
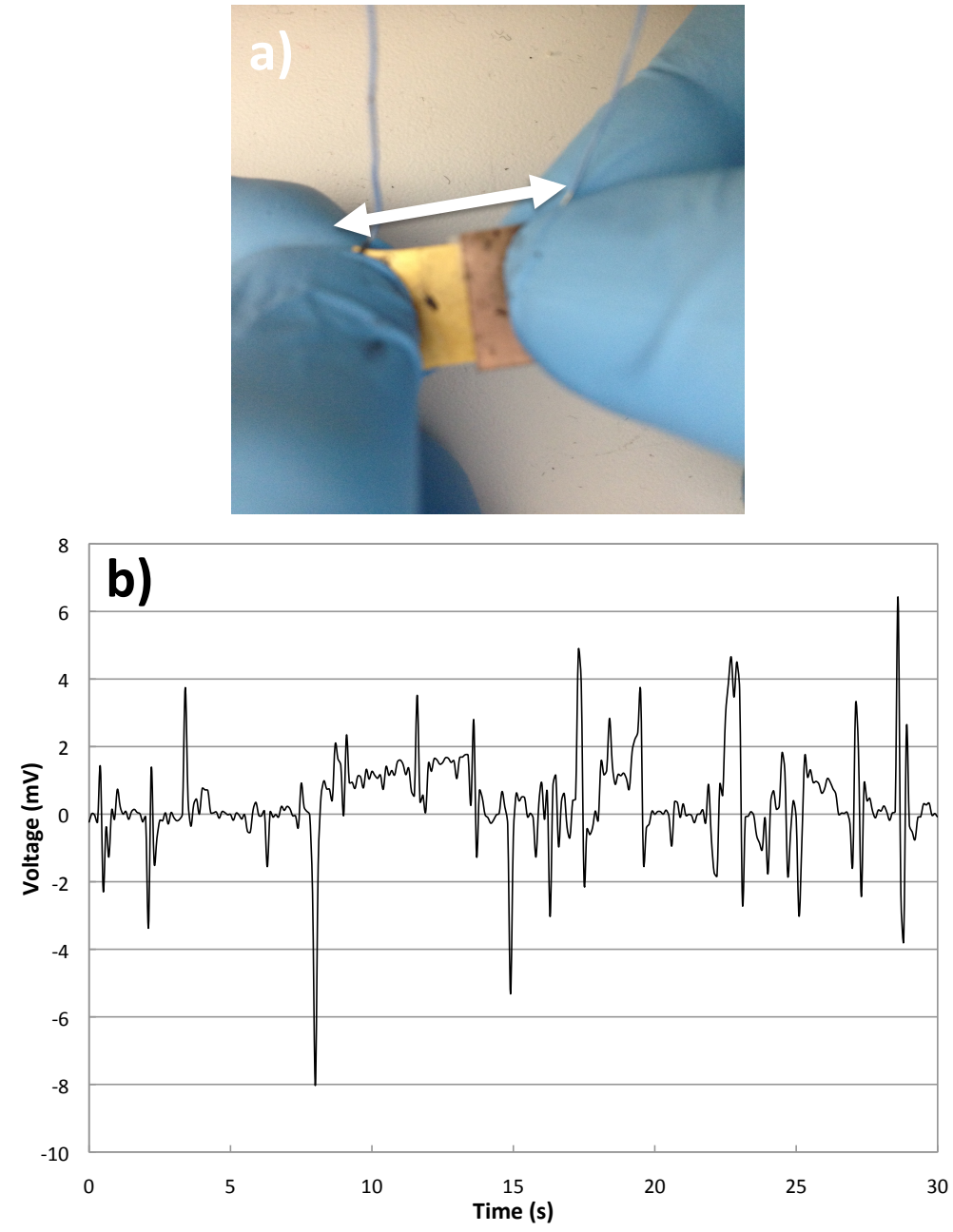

Fig. 3.22 - a) Fingertip tensile loading of PDMS-based device with b) measured electrical output voltage. 


\subsection{Conclusions}

During this work, current designs of $\mathrm{ZnO} \mathrm{NW-based} \mathrm{nanogenerators} \mathrm{were} \mathrm{analyzed} \mathrm{to}$ develop a model system for further mechanical investigations. While numerous designs of glass-based, PEN-based, and PDMS-based devices were fabricated and shown to have measureable electrical outputs, their limited durability and sustained functionality was immediately evident. Fig. 3.23 presents a summary of possible failure mechanisms for Phase II and III devices with their source being either from manufacturing or repeated mechanical loading.

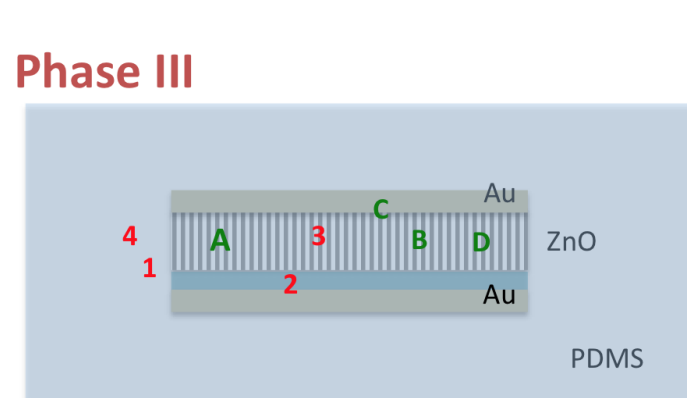

Manufacturing-based Failure Mechanisms

A) Surface Defects

B) Oxygen Vacancies

C) NW Density/Alignment

D) NW/Electrode Contact

Mechanical Loading Failure Mechanisms

1) Electrode Cracking

2) Electrode Delamination

3) Nanowire Fracture

4) Contact-based Wear of Device Components

Fig. 3.23 - Regions of expected failure mechanisms for the Phase III device structure. 


\section{Monotonic mechanical loading studies of indium tin oxide (ITO) electrodes on polyester substrates}

\subsection{Overview}

This chapter describes monotonic mechanical analysis of ITO-coated polyester films. Mechanical loading scenarios were designed to mimic the stresses exerted on electrode films during manufacture and use in contact-based energy harvesting device architectures. Spherical nanoindentation and nanoscratch experiments were performed to characterize the effect of concentrated point loading on durability and film adhesion. Controlled buckling experiments were performed to characterize cracking mechanics on films with etched ITO patterns of various geometries. The work presented in this chapter has been published in Thin Solid Films, doi: 10.1016/j.tsf.2011.08.024. and doi: 10.1016/j.tsf.2013.05.173.

\subsection{Introduction}

During device manufacturing moderate contact loads may be applied to ITO film surfaces, which can result in local surface damage and subsequent loss of device functionality. For the specific case piezoelectric devices, device output is generated through moderate contact loads. These surface loads required to power the device, will eventually lead to flexible electrode, and therefore, device failure. There is little previous research reported on this issue mainly because ITO-coated PET is an unusual system where a hard brittle layer is deposited on a soft compliant substrate resulting in a large modulus mismatch. The response of the soft substrate during contact loading cannot be neglected. When the layer is harder and much thinner than the substrate, the substrate 
effects during nanoindentation are pronounced and the penetration of the indenter is accommodated by pressing the film into the substrate [124].

The thickness of the brittle layer plays a key role in determining potential fracture mode transitions during spherical indentation of brittle coatings on compliant substrates. Ring cracks have been observed in cases where the film thickness lies below $1 \mu \mathrm{m}$ and the contact stresses dominate in the relatively thick substrate region [125]. Also, microscopical observations of spherical impressions, made by typical diamond indenters, on amorphous $\mathrm{SiO}_{2}$ coated polymer substrates revealed ring cracks. The number of cracks increased with increasing load [126].

Indentation and scratch studies of TCO thin films have been mainly focused on films coated on glass base substrates. By using a rigid glass substrate the mechanical properties of the film such as hardness and Young's modulus can be easily extracted. Hardness and elastic modulus of ITO and indium zinc oxide (IZO) films sputtered on glass were measured by nanoindentation and a relation of the sputtering parameters with film mechanical properties was established [127]. In addition, Al-doped $\mathrm{ZnO}$ sputtered on glass found to exhibit increased hardness with higher sputtering power and substrate temperature because of increased microstructural densification and crystallinity. Nanoscratch testing of such films revealed buckling and interfacial delamination failure mechanisms [9]. Nanoindentation and nanoscratch testing, using atomic force microscopy (AFM), of $\mathrm{SnO}_{2}$ films deposited on polycarbonate (PC) and polymethyl methacrylate (PMMA) substrates have been also reported with the aim being mostly towards the scratch protection of the underlying polymer base [128]. 
At this point it is essential to mention that indentation and scratch testing studies using ITO deposited on a polymer substrate do not aid towards extracting the mechanical properties of the ITO coating because the substrate contribution cannot be neglected as in the case of a rigid substrate. They are mainly viewed as an effort to widen the understanding of the failure mechanism(s) involved during the localized contact and sliding of a sphere respectively on the ITO surface. Such an understanding will aid towards designing reliable fabrication and manufacturing processes for the next generation of advanced flexible optoelectronic and piezoelectric energy harvesting devices.

Similarly, there have been somewhat little experimental studies characterizing the mechanical behavior of patterned conducting layers on polymeric substrates. Abdallah et al. [127] investigated the buckle initiation of patterned ITO lines on Arylite substrate. The total number of initiated buckles was found to increase with increasing compressive strain and load time. These buckles were found to emanate from point defects in the ITO layer. Additionally, Chien et al. [129] investigated the mechanical bending capability of amorphous thin film transistors (a-IGZO) on polyimide-based nanocomposite substrates. Chien found that the films can be deformed to a radius of curvature of $3 \mathrm{~mm}$ and still remain functional. Sun et al. [130] investigated the controlled buckling of semiconductor nanoribbons of GaAs and Si on elastomeric polydimethylsiloxane (PDMS) for stretchable electronic applications. They showed that similar structures may provide a route to optoelectronics with an allowable curvature radius down to $\sim 5 \mathrm{~mm}$. Park et al. [131] reported on the experimental and theoretical studies of bending line-patterned silicone nanoribbons on PET substrates. It was observed that the maximum stress occurs at the 
edges due to the patterning definition, while the onset of failure, was found to be equal to $3.04 \%$ strain for a $100 \mathrm{~nm}$ thick film.

The aim of this study was to characterize the response of ITO-coated PET components to spherical indentation, scratching, and controlled buckling. Local coating damage due to indentation and scratching is related to compromised device durability. These monotonic loading scenarios mimic both in service and manufacturing stresses on the hard-on-soft system observed in conductive oxides deposited on polymer substrates.

\subsection{Experimental Methods}

\subsubsection{Materials}

ITO films were deposited on PET substrates (DuPont ST504) using DC magnetron sputtering at room temperature. A sputtering power of approximately $1 \mathrm{~kW}$ was used. Argon and a small quantity of oxygen were the processing gases. The oxygen partial pressure was approximately equal to $2 \mathrm{mPa}$. The deposition rate was $5 \mathrm{~nm} / \mathrm{min}$. The target chemical composition was $\operatorname{In}_{2} \mathrm{O}_{3}: \mathrm{SnO}_{2}=90: 10$. ITO films with thicknesses equal to $50,100,200,250$ and $300 \mathrm{~nm}$ were prepared. Film thicknesses were measured using a stylus profilometer (Veeco Dektak 150) in contact-mode.

Patterned ITO films were fabricated using photolithography and wet chemical etching techniques. Initially, the ITO films were cleaned using isopropyl alcohol and DI water and dried using nitrogen gas. Various patterns were fabricated, a schematic of the electrodes can be seen in Fig. 4.1. 

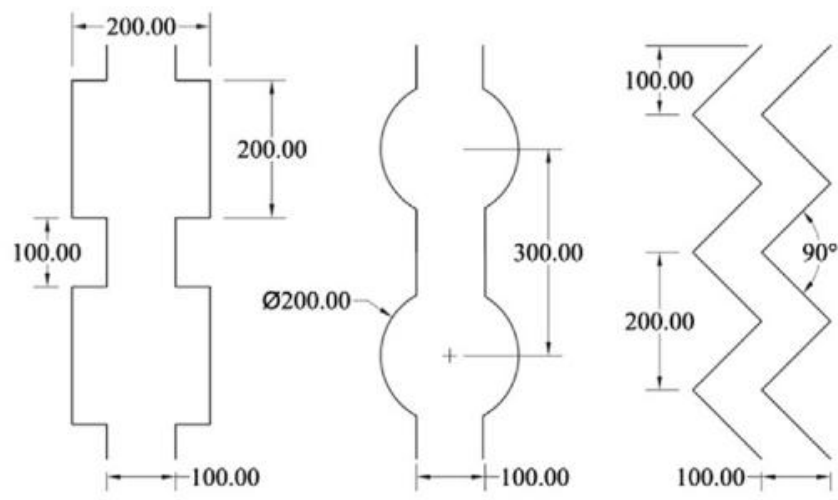

(a)
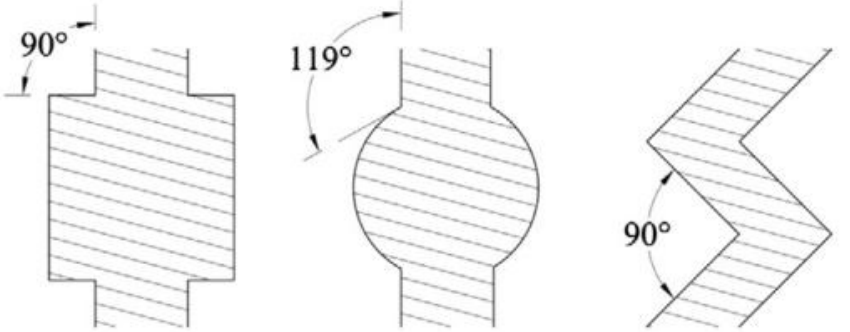

(b)

Fig. 4.1 - Schematic of ITO patterns fabricated using photolithography and wet etching techniques, showing (a) characteristic lengths and (b) angles. Dimensions are in microns and degrees, respectively.

The specimen surface was spin-coated at $2000 \mathrm{rpm}$ for 1 min with AZ 3330 photoresist and pre-baked at $90{ }^{\circ} \mathrm{C}$ for 1 minute. After exposure through a photomask and post-bake at $90{ }^{\circ} \mathrm{C}$, the resist was developed in an AZ 3330 developer solution. The specimens were then etched in $2 \%$ oxalic acid $\left(\mathrm{C}_{2} \mathrm{H}_{2} \mathrm{O}_{4}\right)$ at an approximate etch rate of $10 \mathrm{~nm} / \mathrm{min}$.

Finally, the remaining photoresist was removed using acetone followed by both cleaning in DI water and nitrogen blowing.

\subsubsection{Nanoindentation}

Spherical indentation testing was performed using a CSM Instruments Ultra Nano

Hardness Tester. The ITO-coated PET samples were glued on glass microscope slides and mounted to the indenter's stage. The radius of the spherical diamond indenter, used to promote cracking on the ITO layer, was equal to $20 \mathrm{~m}$. Various loads up to $200 \mathrm{mN}$ were 
applied. The loading/unloading rate was equal to $100 \mathrm{mN} / \mathrm{min}$. A pause of $10 \mathrm{~s}$ was used between loading and unloading to control viscoelastic creep effects. After testing, indents on the ITO surface were investigated using a built-in optical microscope.

\subsubsection{Scratch Testing}

The scratch test is used to assess the adhesion of coatings bonded to substrates [132]. Scratch testing is performed using a Nano Scratch Tester (CSM Instruments). The ITO coated PET samples were glued on glass microscope slides and mounted to the scratch tester's stage. A diamond indenter with a $5 \mu \mathrm{m}$ radius is drawn across the ITO film surface with a loading rate equal to $5 \mathrm{mN} / \mathrm{min}$. The tip radius is selected in order to simulate the sharp contact conditions of typical LED chip edges on flexible planes during fabrication of chip-on-flex devices. A total scratch length of $0.5 \mathrm{~mm}$ is generated with the end load being $5 \mathrm{mN}$. The failure modes that occur at some critical loads are confirmed using a built-in optical microscope. Also, a built-in atomic force microscope (AFM) operating in tapping-mode is used to observe the film's scratched areas.

\subsubsection{Controlled Buckling of Patterned Films}

Controlled buckling experiments were conducted using a custom-built, manually operated apparatus (description in Chapter 2). Sample displacement measurements were taken using a side-view digital imaging system (Celestron Digital Microscope) and they were analyzed using image analysis software (ImageJ NIH USA). The buckling radius and the resulting strain were calculated according to the procedure described by Chen $e t$ al. [90]. In situ optical microscopy images were taken at small increments of applied 
strain in order to determine both tensile and compressive crack onset strains and to monitor the crack propagation. In addition, in-situ electrical resistance changes were monitored using a Fluke 45 dual display multimeter. Scanning electron microscopy (SEM) was used in order to ex situ investigate the scratch failure mechanisms and the indentation imprints in more detail.

\subsection{Results and Discussion}

\subsubsection{Tensile Testing of Films}

The critical-onset strain (COS) at which the ITO-coated PET starts to crack was determined by tensile testing of the composite film. A sudden increase in resistance was observed at around $2.25 \%$ tensile strain for the 200 /sq sample and $1.75 \%$ for the 100 /sq sample .

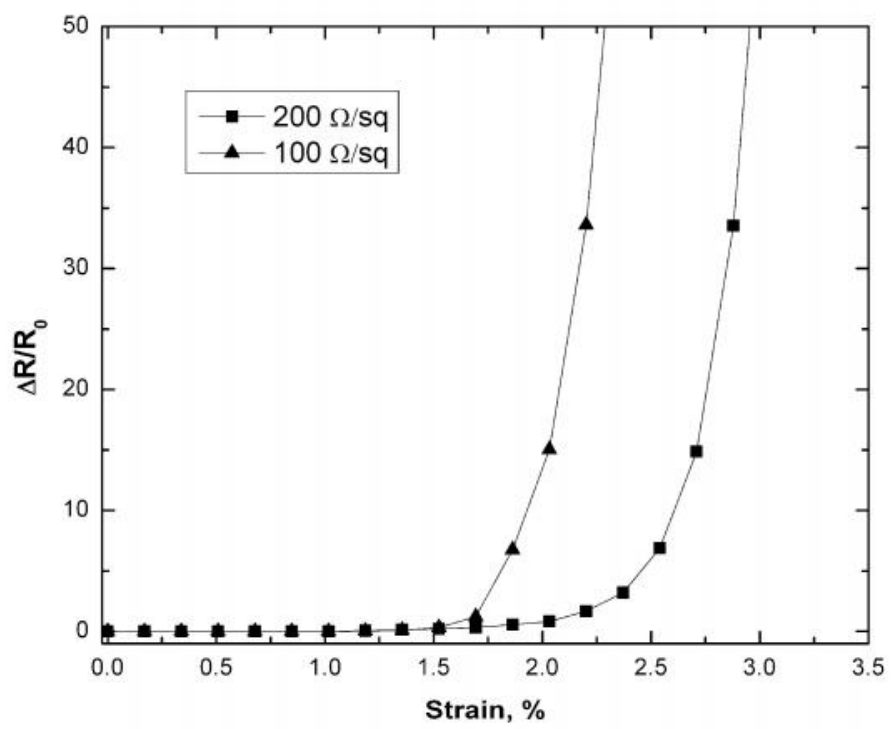

Fig. 4.2 - Electromechanical behavior of ITO-coated PET with sheet resistances of 100 and 200 /sq.

The difference in the two crack-onset values is due to the difference in film thickness of the two samples. These critical strains are consistent with high quality sputter deposited films without macroscale defects [132]. The 200 /sq sample is thinner than its 100 / sq 
counterpart. Thinner ITO, coated on the same polymer substrate, shows a higher criticalonset strain than thicker specimens. At low tensile strains (below 6\%) the ITO failure mechanism is cracking, perpendicular to the tensile direction. Above $6 \%$ tensile strain the ITO delaminates from the substrate and also cracks, as shown in Fig. 4.3.

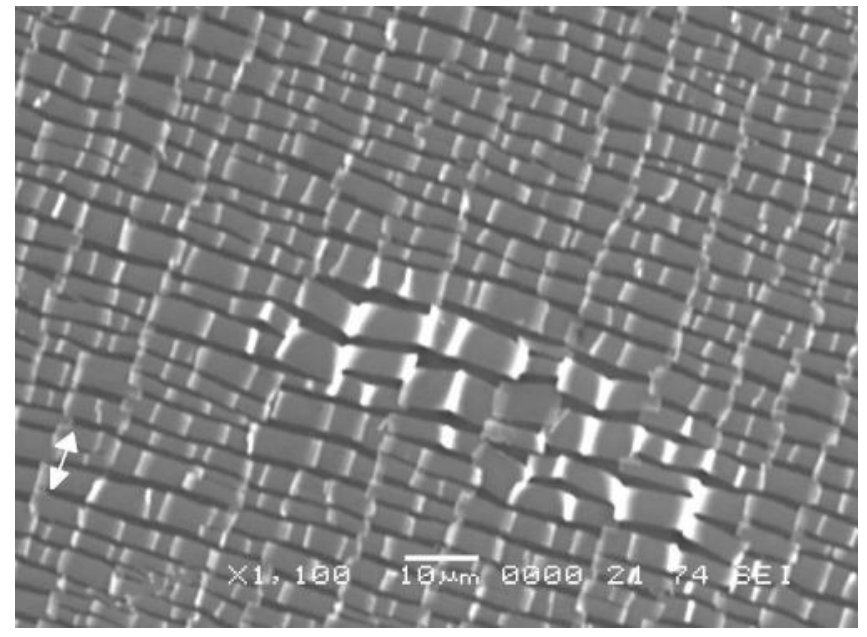

Fig. 4.3 - ITO cracking and delamination of an ITO-coated PET sample tensile tested above 6\% strain. Arrows indicate force direction.

\subsubsection{Spherical Indentation}

Spherical indentation force-displacement curves for various thicknesses of ITO coatings is presented in Fig. 4.4. 


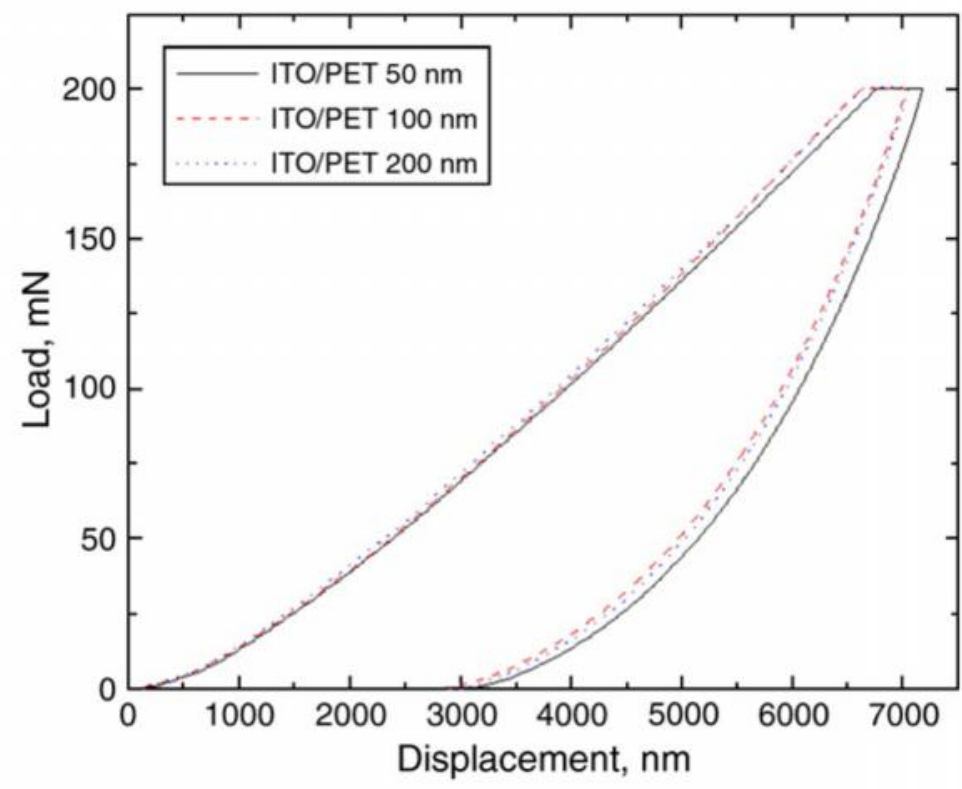

Fig. 4.4 - Load displacement spherical indentation curves for ITO coatings sputtered on polyester substrates.

The influence of the polymeric substrate dominated the overall response of the system under spherical indentation loading. The loading segment of the curve was the same for all different thicknesses and a slight difference was observed in the unloading portion of the curve. During unloading, the PET coated with the $50 \mathrm{~nm}$ thick film tends to permanently deform slightly more than its thicker counterparts.

A more interesting aspect of the indentation study related to the initiation and propagation of failure mechanisms and cracking patterns on the surface of the ITO films. A typical failure mode sequence observed in this study is presented in Fig. 4.5 for the case of a $100 \mathrm{~nm}$ thick ITO coating. 

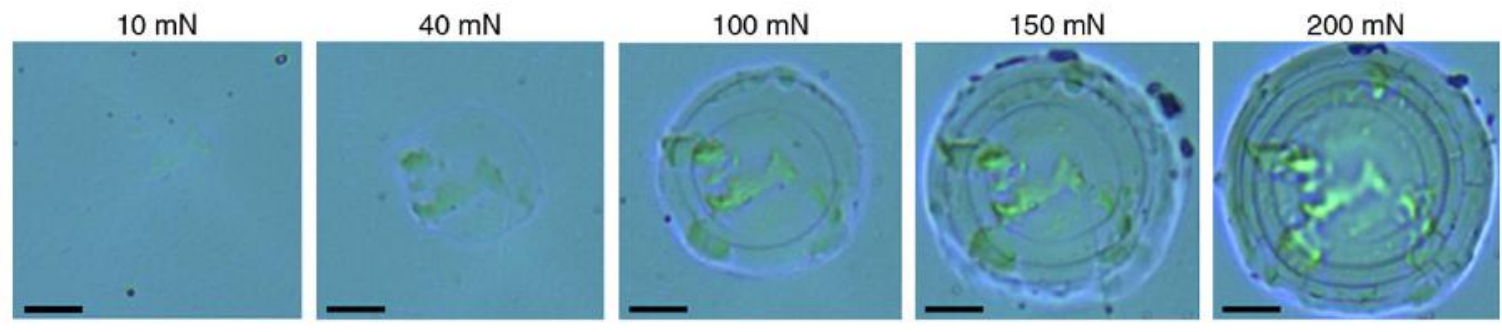

Fig. 4.5 - Failure pattern evolution for a $100 \mathrm{~nm}$ thick ITO coating as normal spherical loading increases. The scale bar is equal to $5 \mu \mathrm{m}$ for all images.

As observed from the optical images taken ex situ, at $40 \mathrm{mN}$ load, the first crack was formed on the ITO surface. This load was considered the critical load for crack onset. Also, a plastically deformed area, due to the contact with the indenter, within the crack circumference, was observed. When the load increased to $100 \mathrm{mN}$, ring crack formation was observed. Additionally, the plastically deformed area appeared more pronounced due to the increased load. It was noted that a pile-up region on the outer circumference also formed. The pile-up region leads to localized coating delamination. This denotes weak coating/polymer adhesion at localized spots. When the load increased to $150 \mathrm{mN}$, the ring crack pattern further evolved. A secondary crack formation was observed when the load further increased to $200 \mathrm{mN}$. These radial cracks were observed to bridge the primary ring cracks. Fig. 4.6 presents an SEM image of a region of a spherical indent where primary ring cracks and a secondary radial crack have formed. It was evident that the secondary crack was bridging the ring cracks. 


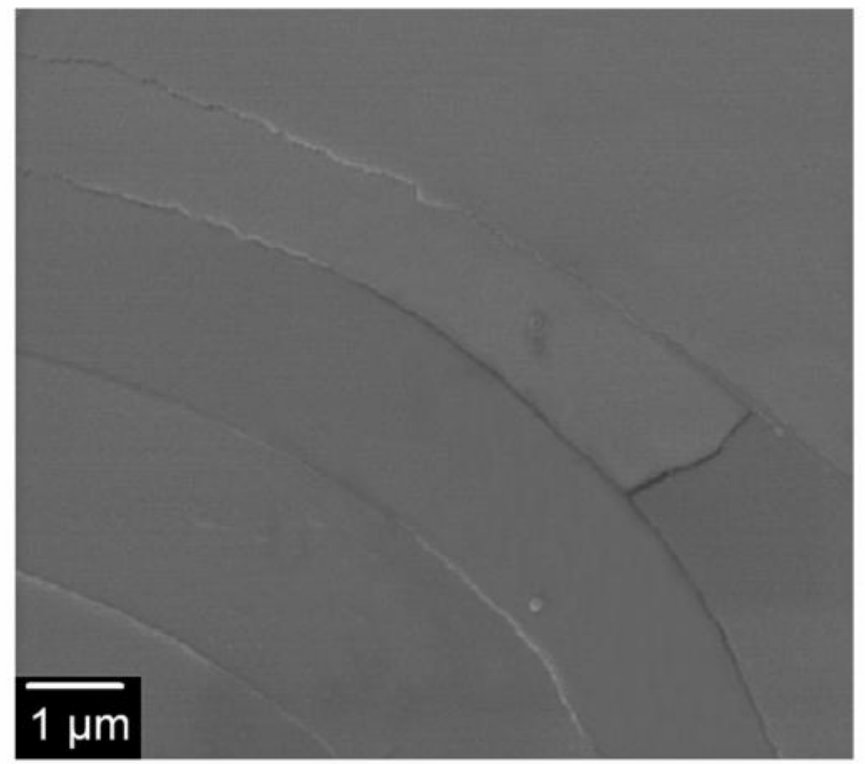

Fig. 4.6 - Scanning electron microscope image of a part of a spherical indent on a $200 \mathrm{~nm}$ thick ITO coated PET, under a $200 \mathrm{mN}$ load.

Fig. 4.7 presents optical images of the spherical indents that were created on the ITO surfaces under different loads ranging from 40 up to $200 \mathrm{mN}$. 


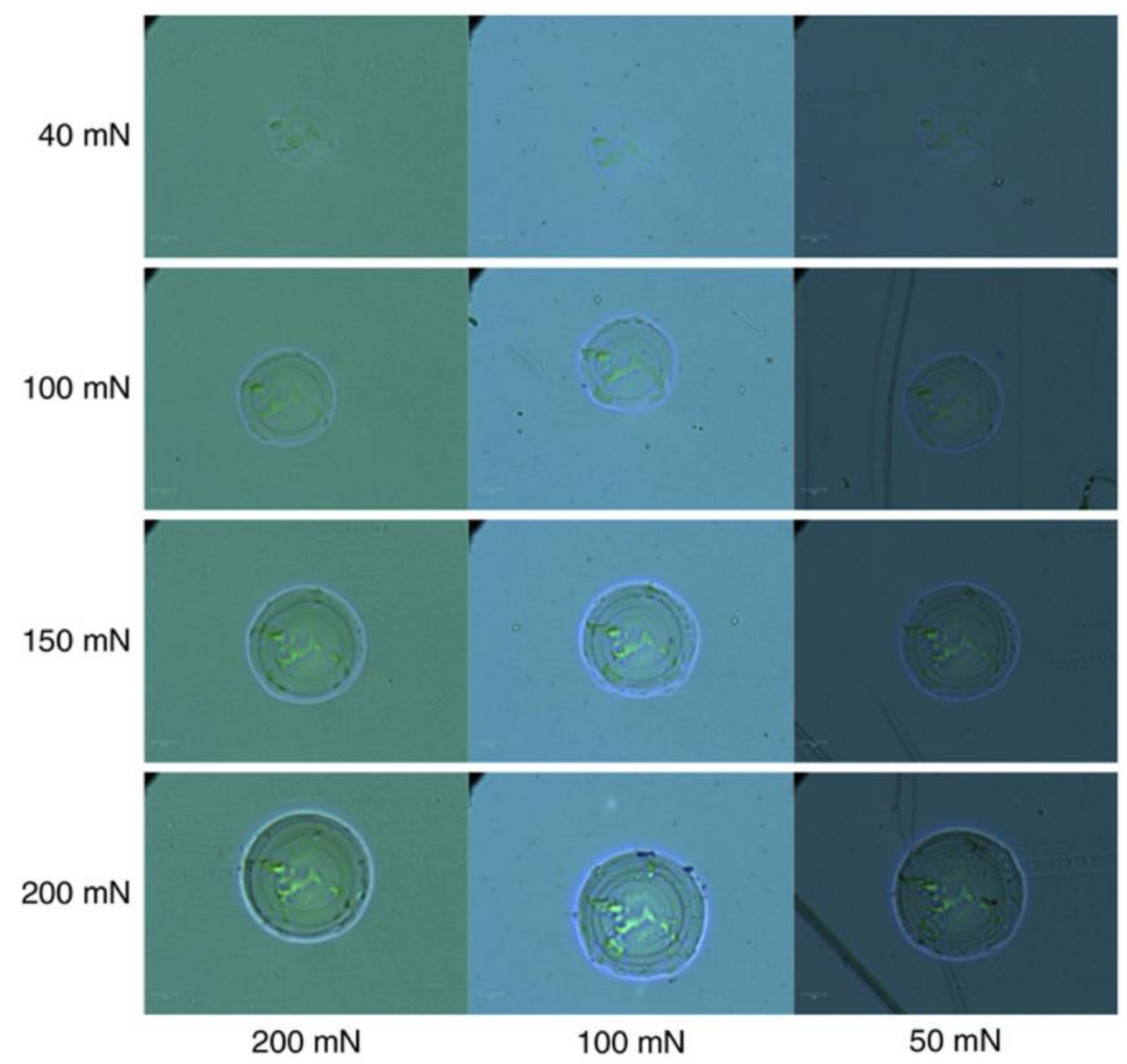

Fig. 4.7 - Ring crack patterns on ITO surfaces for different normal loads and film thicknesses.

Three ITO coating thicknesses were considered. For all thicknesses investigated the first ring crack forms at $40 \mathrm{mN}$ of normal spherical loading. Concentric ring cracks then formed as the load increased, following a similar pattern for all three thicknesses.

In general, the mechanism of crack formation was as follows. During loading, ring cracks form outside of the contact region at some threshold which was equal to $40 \mathrm{mN}$. Above such threshold the contact tensile stresses cause substrate deformation to increase and the number of ring cracks increases. At higher loads, secondary cracks forms due to 
increased transverse contact stresses.

The relation of the measured indent imprint radius ( $\left.a^{\prime}\right)$, after testing, with applied normal load is presented in Fig. 4.8.

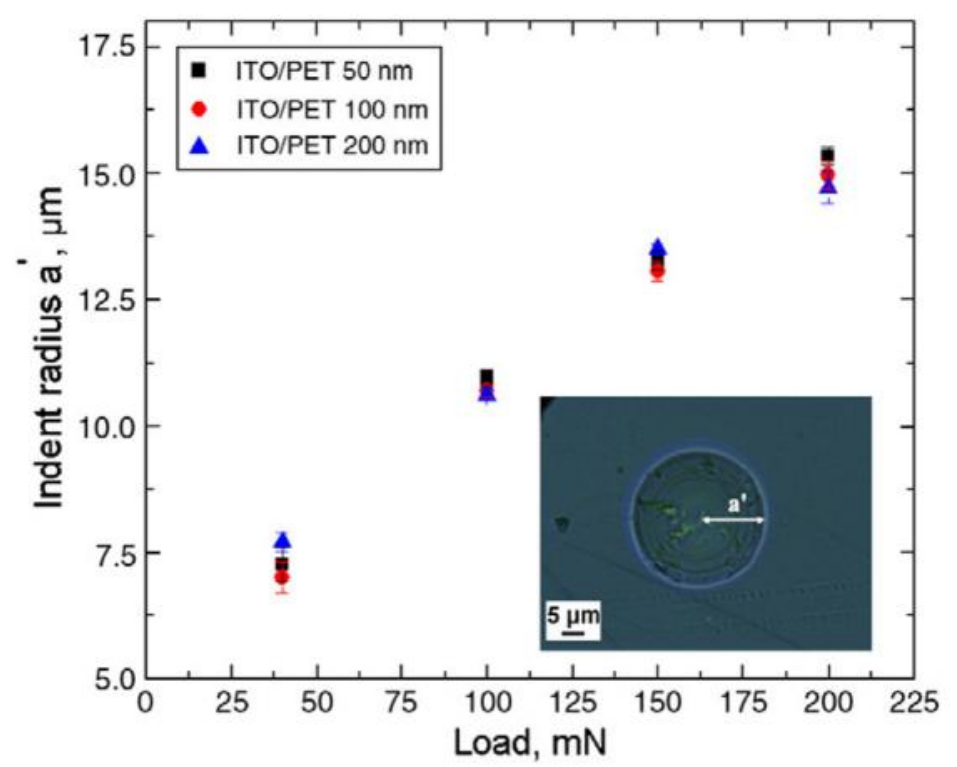

Fig. 4.8 - Indent imprint radius versus applied load for coatings with different thicknesses. Inset optical image shows and indent of $50 \mathrm{~nm}$ thick coating subjected to a $150 \mathrm{mN}$ load (scale bar is equal to $5 \mu \mathrm{m}$ ).

It was observed that the indent imprint radius increased linearly with increasing load.

Since the applied normal loads were relatively high, for the coating/substrate thickness ratios investigated, independence of a' on coating thickness was distinguished. The elastic effects of the underlying polymer substrate dominated the mechanical response. For a normal load of $40 \mathrm{mN}$, the measured indent radius was around $7.25 \mu \mathrm{m}$ and increased linearly to approximately $15 \mu \mathrm{m}$ when the applied load reached $200 \mathrm{mN}$.

Hertzian elastic contact theory cannot be applied in this case since the recovery of the ceramic coated polymer surface was not totally elastic and a residual plastic deformation portion was observed after unloading [134]. Therefore, elasto-plastic deformation 
conditions must be considered [135]. The contact depth for elasto-plastic penetration is given by Eq. (1) below.

$$
h_{c}=h_{r}+\frac{\left(\begin{array}{ll}
h_{t} & h_{r}
\end{array}\right)}{2}
$$

Where $h_{c}$ is the contact depth for elasto-plastic deformation, $h_{r}$ is the residual depth of the indentation and $\mathrm{ht}$ is the total depth of the indentation. Both $\mathrm{h}_{\mathrm{r}}$ and $\mathrm{ht}$ can be determined from load-depth indentation curves.

The average contact pressure (Paverage) at maximum load is shown in Eq. (2).

$$
P_{\text {average }}=\frac{F}{2}
$$

Where $\mathrm{F}$ is the applied normal load and is contact radius. The contact radius is given by Eq. (3).

$$
=\sqrt{h_{c}\left(R \quad h_{c}\right)}
$$

Where $\mathrm{R}$ is the radius of the spherical indenter.

The average contact pressure is then given by the following equation.

$$
P_{\text {average }}=\frac{F}{h_{c}\left(R \quad h_{c}\right)}
$$

The relation of the average contact pressure with the applied normal load, considering elasto-plastic deformation conditions, is shown in Fig. 4.9. 


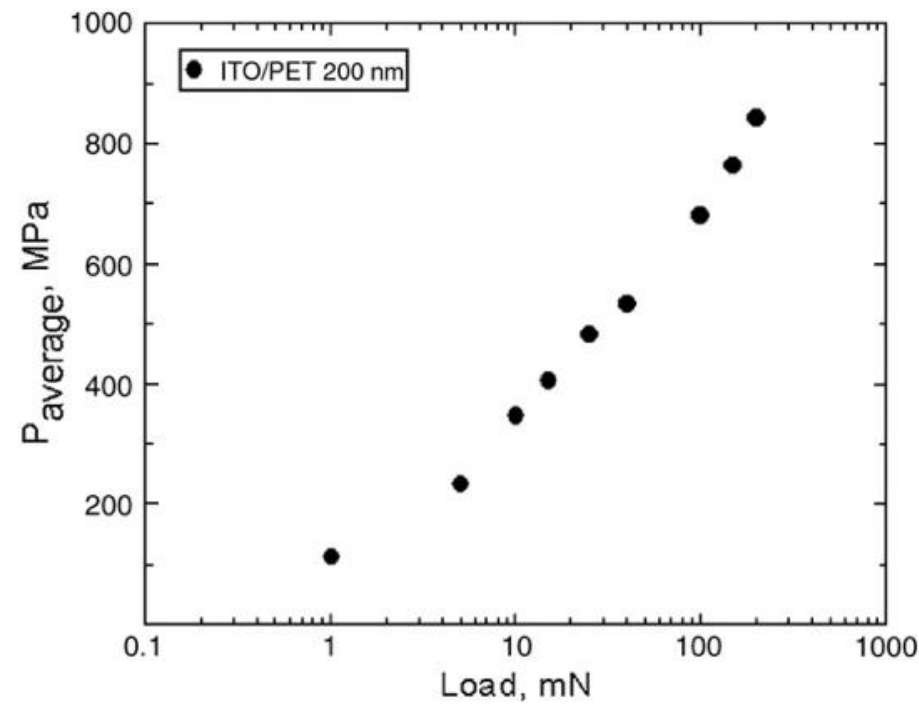

Fig. 4.9 - Semi-log plot of average contact pressure versus applied load for ITO coated PET. The thickness of the ITO coating is $200 \mathrm{~nm}$.

For loads as low as $1 \mathrm{mN}$ the contact pressure was equal to $113 \mathrm{MPa}$, a value, which is above the typical yield point of a PET film [26]. As loading increased the contact pressure increased and reached a maximum of $842.7 \mathrm{MPa}$ for $200 \mathrm{mN}$ applied normal load.

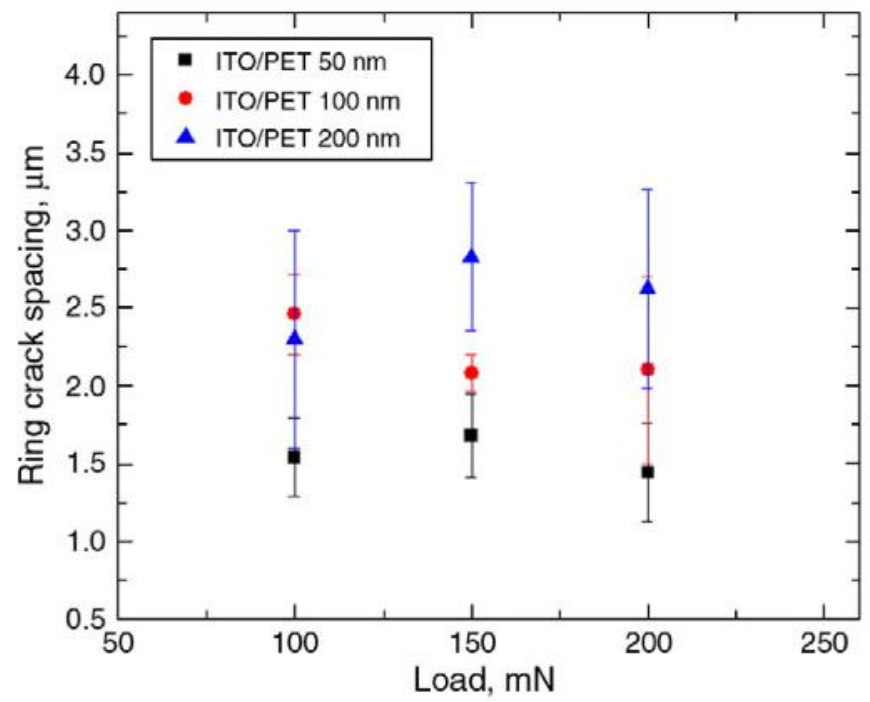

Fig. 4.10 - Ring crack spacing versus applied normal load for 50, 100, and $200 \mathrm{~nm}$ thick ITO coatings sputtered on PET substrates. Error bars represent stand deviation of the data.

It was observed that thicker ITO coatings exhibit higher crack spacing particularly for 
higher applied loads. As thickness decreases a tendency for the coating to fragment in smaller parts was noted. This was consistent with previous studies, of tensile loaded brittle $\mathrm{SiO}_{\mathrm{x}}$ coatings on PET substrates, where thinner coatings exhibited higher crack density [137].

\subsubsection{Scratch Testing}

The scratch test was used to investigate thin film scratch failure mechanisms that occur when a diamond stylus is drawn across the ITO-coated polyester surface under an increasing normal load. Fig. 4.11a shows a panoramic optical image of the total scratch length of a $50 \mathrm{~nm}$ thick ITO coating on PET with the direction of sliding being from left to right. There are two failure modes observed using optical microscopy. Through thickness coating cracking was the first failure mechanism as shown in Fig. 4.11b. The second mechanism was coating spallation as shown in Fig. $4.11 \mathrm{c}$. Both these mechanisms were commonly observed in hard coating-soft substrate systems [132].

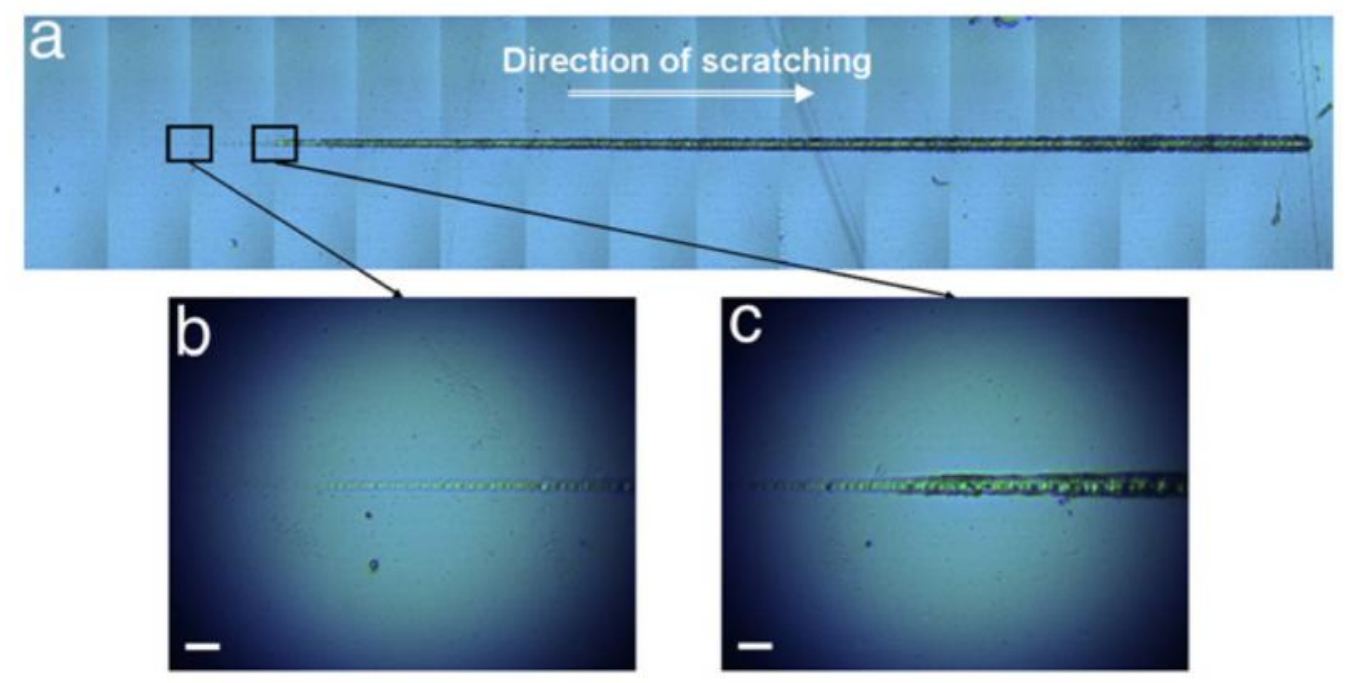

Fig. $4.11-$ a) Typical panoramic view of a scratched ITO surface. Coating thickness is equal to $50 \mathrm{~nm}$ and the total scratch length is equal to $0.5 \mathrm{~mm}, \mathrm{~b}$ ) optical image showing the initiation of through thickness cracking, c) optical image showing the initiation of coating spallation. Both optical images have scale bars equal to $5 \mu \mathrm{m}$. 
Furthermore, Fig. 4.12a shows a two-dimensional tapping-mode AFM image showing brittle coating cracking. The thickness of the film was equal to $250 \mathrm{~nm}$. It is observed that brittle tensile cracks developed perpendicular to the sliding direction. The cracks developed behind the indenter due to the tensile stresses present at the surface. Coating buckling spallation is presented in Fig. 4.12b.
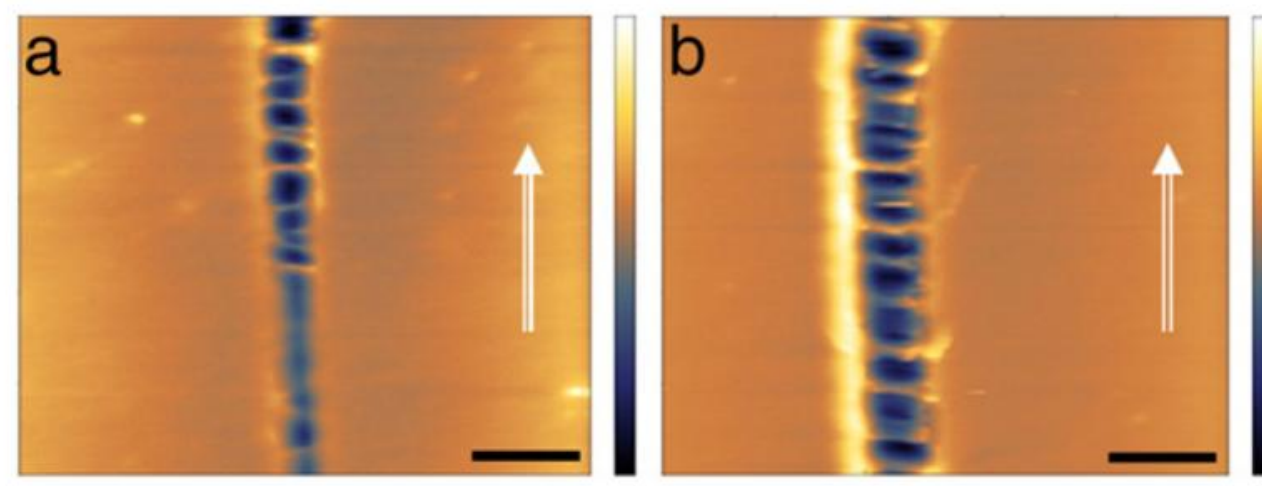

Fig. $4.12-$ a) Typical tapping-mode AFM image showing coating cracking: vertical range $=348 \mathrm{~nm} \mathrm{~b}$ ) tapping-mode AFM image showing coating buckling spallation; vertical range $=830 \mathrm{~nm}$. The coating has a thickness equal to $250 \mathrm{~nm}$. Arrows indicate the direction of scratching. Both micrographs have scale bars equal to $5 \mu \mathrm{m}$.

Buckling spallation mainly occurred ahead of the indenter. Fig. 4.13, presents an SEM image of a damaged ITO surface due to spallation.

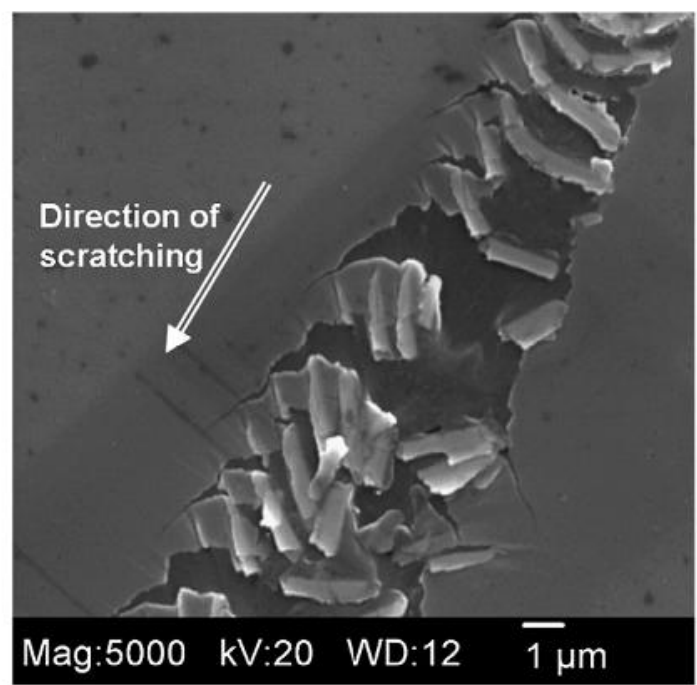

Fig. 4.13 - SEM image of a $200 \mathrm{~nm}$ thick ITO coating showing spalled areas and interfacial failure during scratch testing. 
The compressive stresses developing ahead of the moving indenter caused the coating to buckle and some spalled areas were visible. The deformed underlying polymer substrate was also visible indicating coating-substrate interfacial failure.

Finally, Fig. 4.14 presents a graph relating the critical load, needed to cause coating cracking and spallation, with coating thickness.

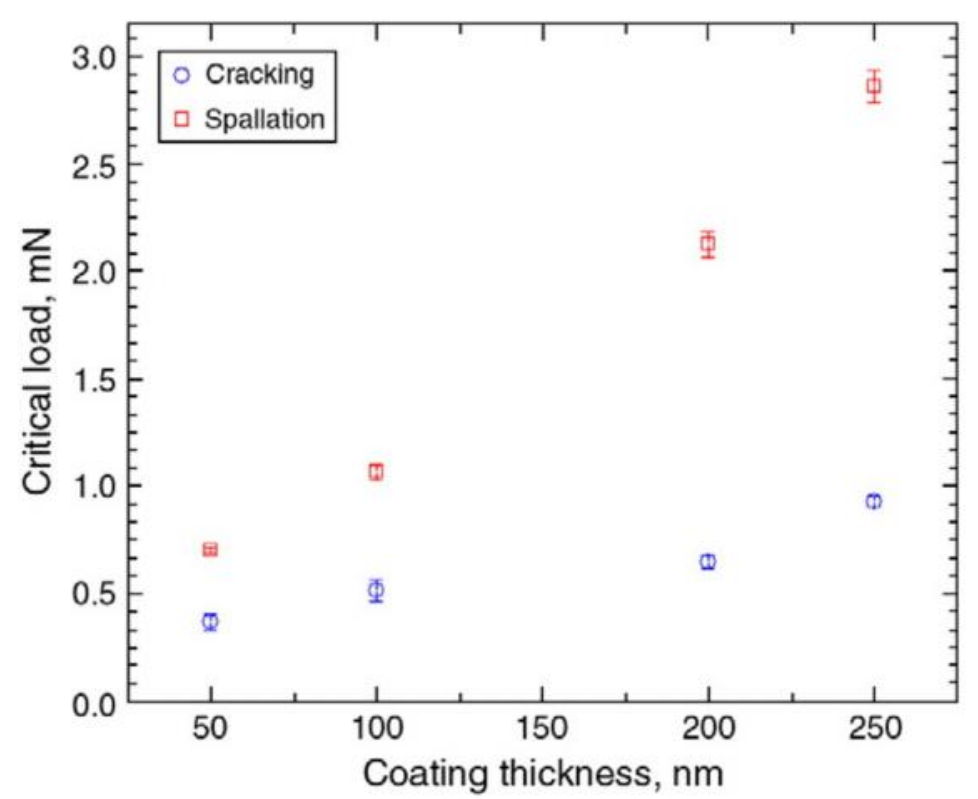

Fig. 4.14 - Graph showing the relation of applied critical load and coating thickness for cracking and spallation failure modes during scratch testing.

It was observed that as coating thickness increased an increase in critical load occurred.

This increase was not very pronounced in the case of the ITO cracking event. However, it was more pronounced in the case of buckling spallation. This was probably due to the fact that thicker films allow more plastically piled-up material to form ahead of the moving indenter before they buckle. The load range for coating cracking was between 0.3 to $0.9 \mathrm{mN}$ for the different thicknesses, whereas for coating spallation the range exhibited was between 0.7 to $2.8 \mathrm{mN}$. Therefore, a tangential load range between $0.3-0.9 \mathrm{mN}$, 
depending on coating thickness, can be regarded as the absolute maximum limit for application of scratch contact loads during chip-on-flex device fabrication.

\subsubsection{Controlled Buckling}

To characterize the effect of lithographic patterning on ITO-coated PET film durability, samples were strain tested using a custom-built controlled buckling apparatus. During experiments, ITO coatings were placed under both tensile and compressive strains up to $6 \%$ while films were observed in situ using optical microscopy and electrical resistance measurements to quantify $\operatorname{COS}$ values. Both techniques were used to determine $\operatorname{COS}$ to increase test accuracy. While optical measurements of COS are considered accurate, they were used primary as a supportive comparison to the more tightly controlled normalized change in resistance metric.

For electrical measurements, a sudden increase of $>10 \%$ was used as a distinction for when the COS had been reached for a given sample [138]. Figs. 4.15 and 4.16 present characteristic graphs of normalized change in resistance experiments on patterned ITOcoated PET samples under tensile and compressive buckling strain, for both small and large patterns, respectively.

(a)

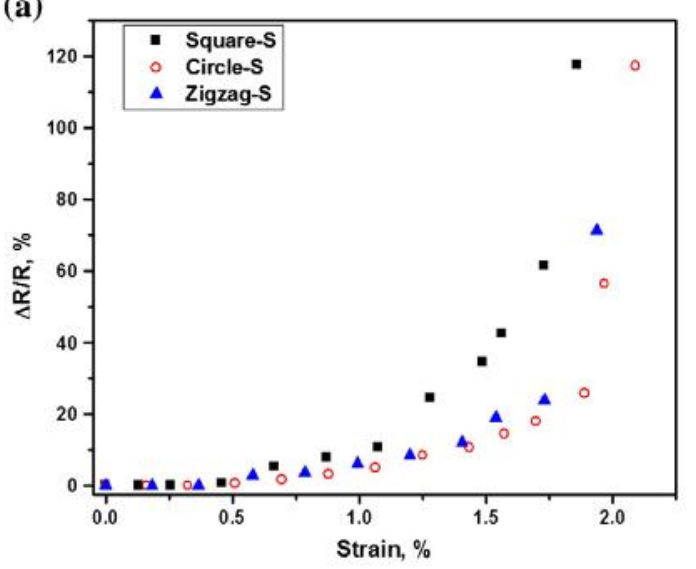

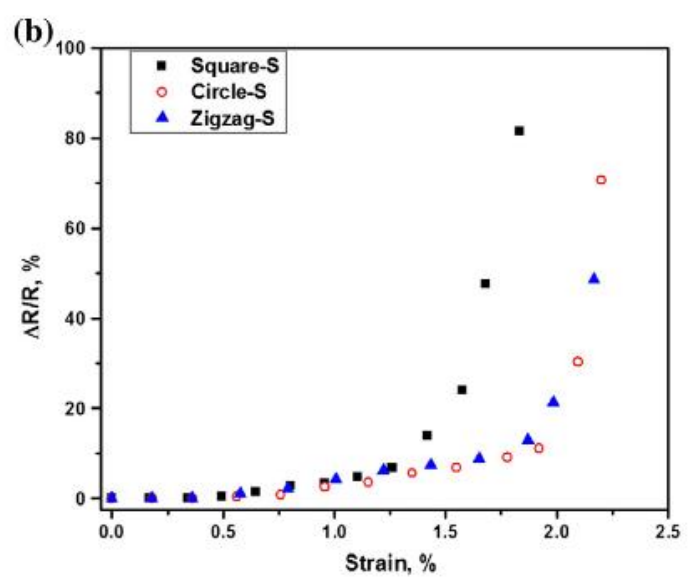


Fig. 4.15 - Normalized electrical resistance versus applied strain for smaller size patterned ITO-coated PET films under a) tension and b) compression

(a)

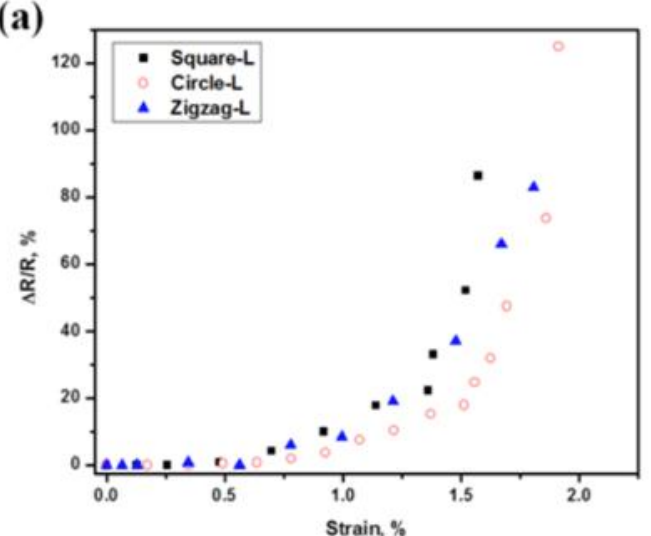

(b)

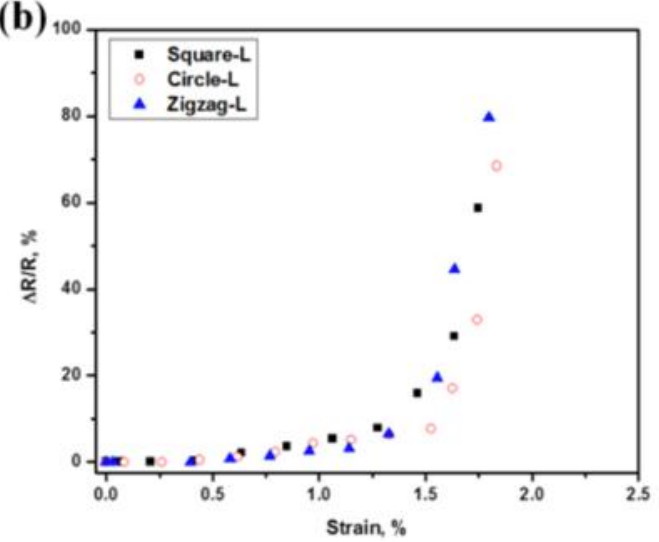

Fig. 4.16 - Normalized electrical resistance versus applied strain for larger size patterned ITO-coated PET films under a) tension and b) compression

A more rapid increase in electrical resistance was observed in the case of the square electrode patterns; occurring at a strain of $1.25 \%$. This was observed for both conditions of tensile and compressive buckling strain. Fig. 4.17 summarizes the COS values for each electrode pattern, determined using normalized resistance measurements.

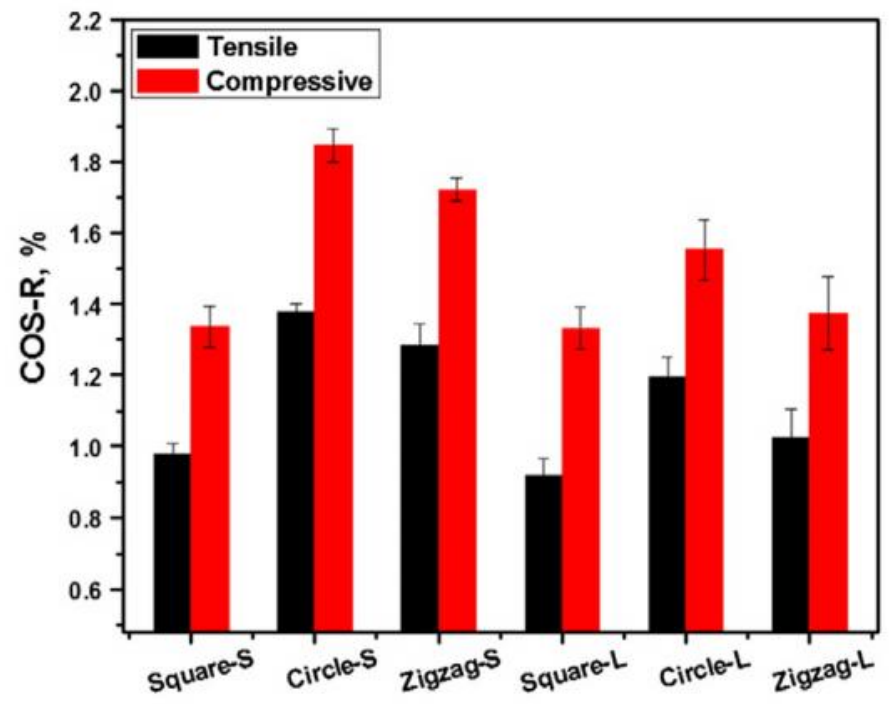


Fig. 4.17 - Crack onset strain from change in electrical resistance monitoring of ITO-coated PET films. It is apparent that patterned electrodes loaded in tensile buckling strain will fail at a lower applied strain than in compressive loading. In particular, the COS for the smaller circular patterns was measured to be $1.38 \%$, while the compressive COS value was $1.85 \%$. While these values were determined to be comparable to virgin, unpatterened ITO-coated PET films, the higher COS values in the smaller patterns were contributed to the reduced volume of brittle material present, and therefore reduced likelihood of intrinsic defects present in the film and reduced etching border defects, which can induce cracking $[89,139]$.

It was also observed that the COS values for under tensile and compressive buckling strains for circular and zigzag patterned electrodes were higher than those for square patterns. This was attributed to the number of stress concentrating corners found in the square electrode pads. Additionally, the square patterns, both large and small, have the highest respective material volumes of ITO-coating, and therefore a higher statistical presents of crack-inducing intrinsic defects.

\subsubsection{Microscopy}

Figs. 4.18 and 4.19 present typical optical micrographs of cohesive failure progression under tensile and compressive controlled buckling conditions, respectively. 

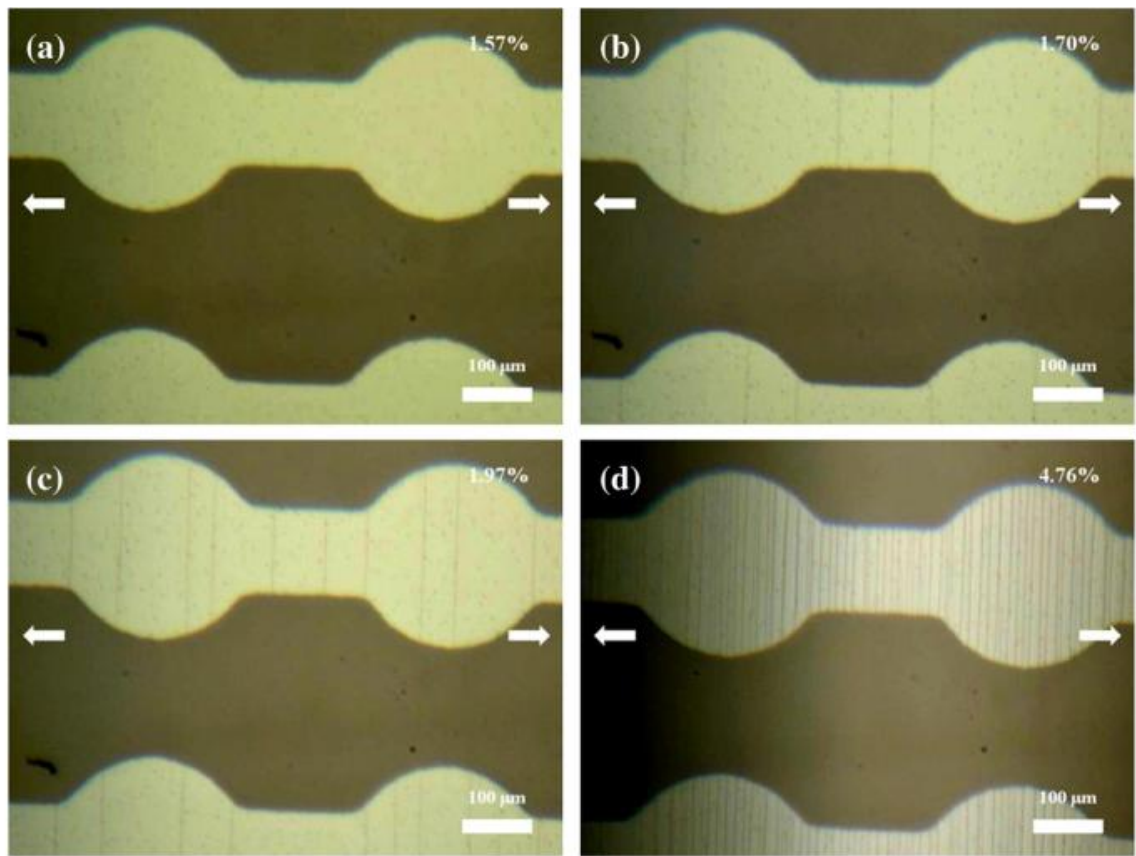

Fig. 4.18 - Optical microscopy images of circular patterned ITO-coated PET films under tensile buckling deformation mode showing cracking initiation and propagation up to $6 \%$ strain. Arrows indicate the buckling direction.
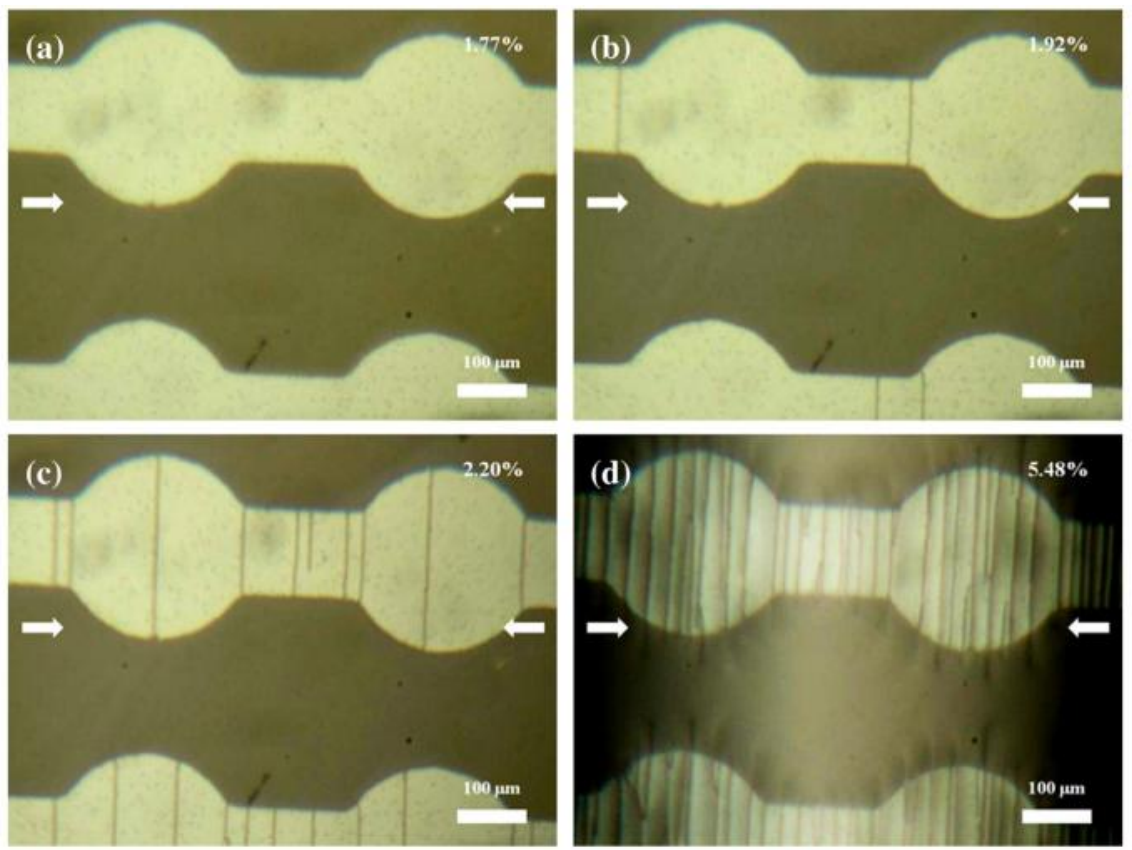

Fig. 4.19 - Optical microscopy images of circular patterned ITO coated PET films under compressive buckling deformation showing cracking initiation and propagation up to $6 \%$ strain. Arrows indicate the buckling direction.

Cohesive cracking perpendicular to the strain direction occurs when the brittle electrode reaches a threshold value. Amount of cracking is directly proportional to the applied 
external strain.

Similarly to circular patterns, cohesive cracking failure is shown in micrographs of square patterned electrodes; presented in Figs. 4.20 and 4.21. Extensive perpendicular cracking can be observed in samples loaded to $4 \%$ tensile and 5.61\% compressive strain, respectively.
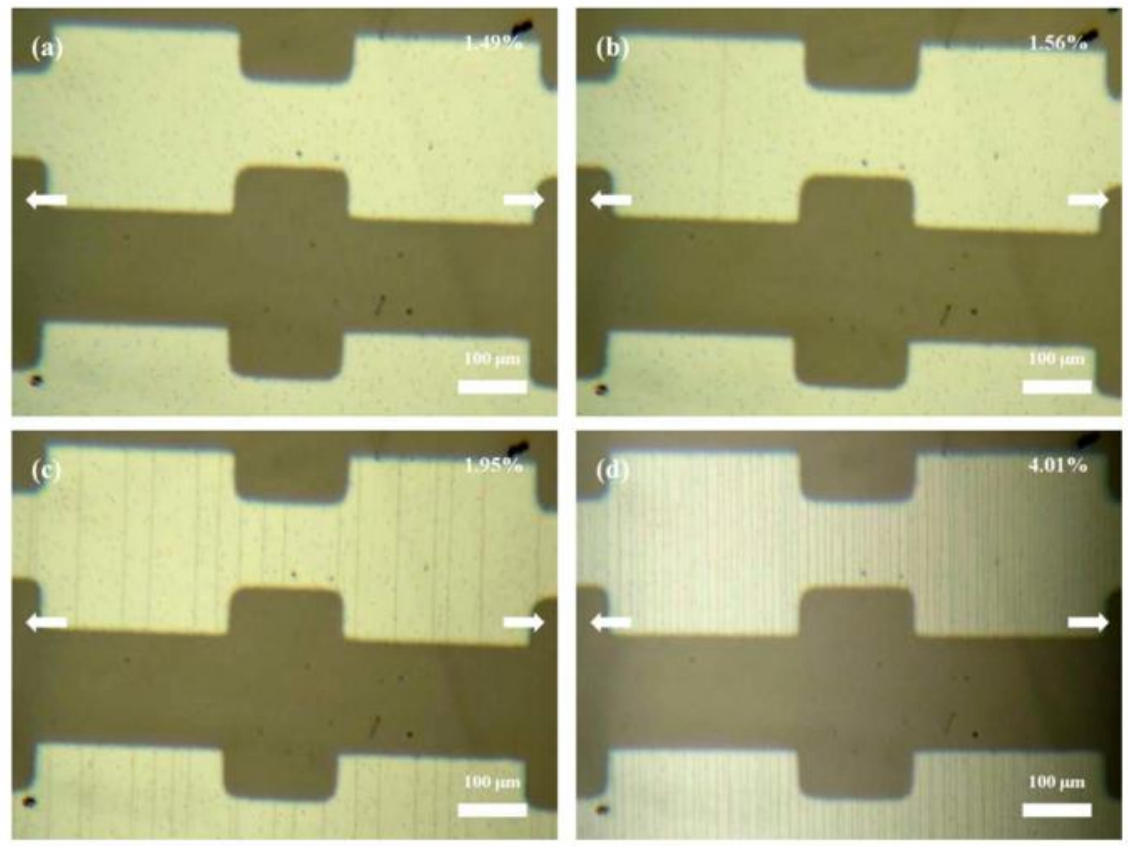

Fig. 4.20 - Optical microscopy images of square patterned ITO-coated PET films under tensile buckling deformation mode showing cracking initiation and propagation up to $6 \%$ strain. Arrows indicate the buckling direction. 

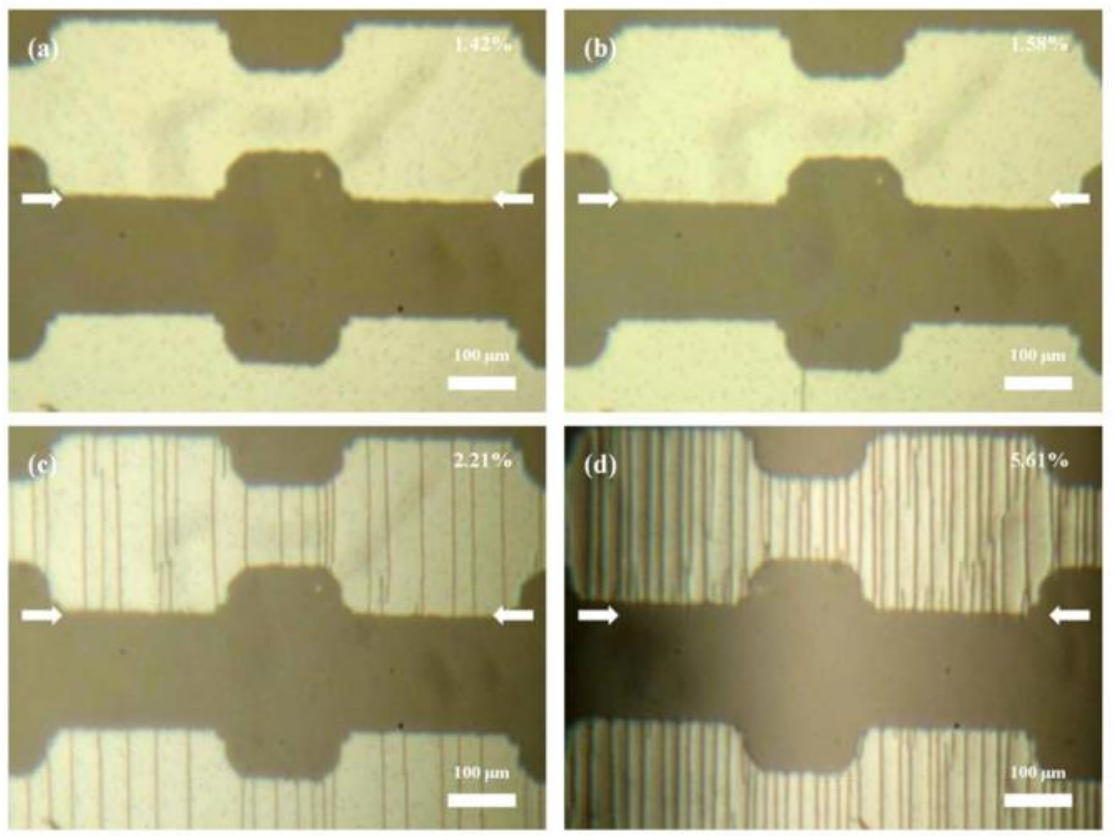

Fig. 4.21 - Optical microscopy images of square patterned ITO-coated PET films under compressive buckling deformation mode showing cracking initiation and propagation up to $6 \%$ strain. Arrows indicate the buckling direction.

Fig. 4.22 and 4.23 represent zigzag patterned samples under various tensile and compressive buckling strains. Fig. $4.23 \mathrm{~b}$ and Fig. $4.23 \mathrm{c}$ are good examples of cracks initiated by sharp corner stress concentrators. 

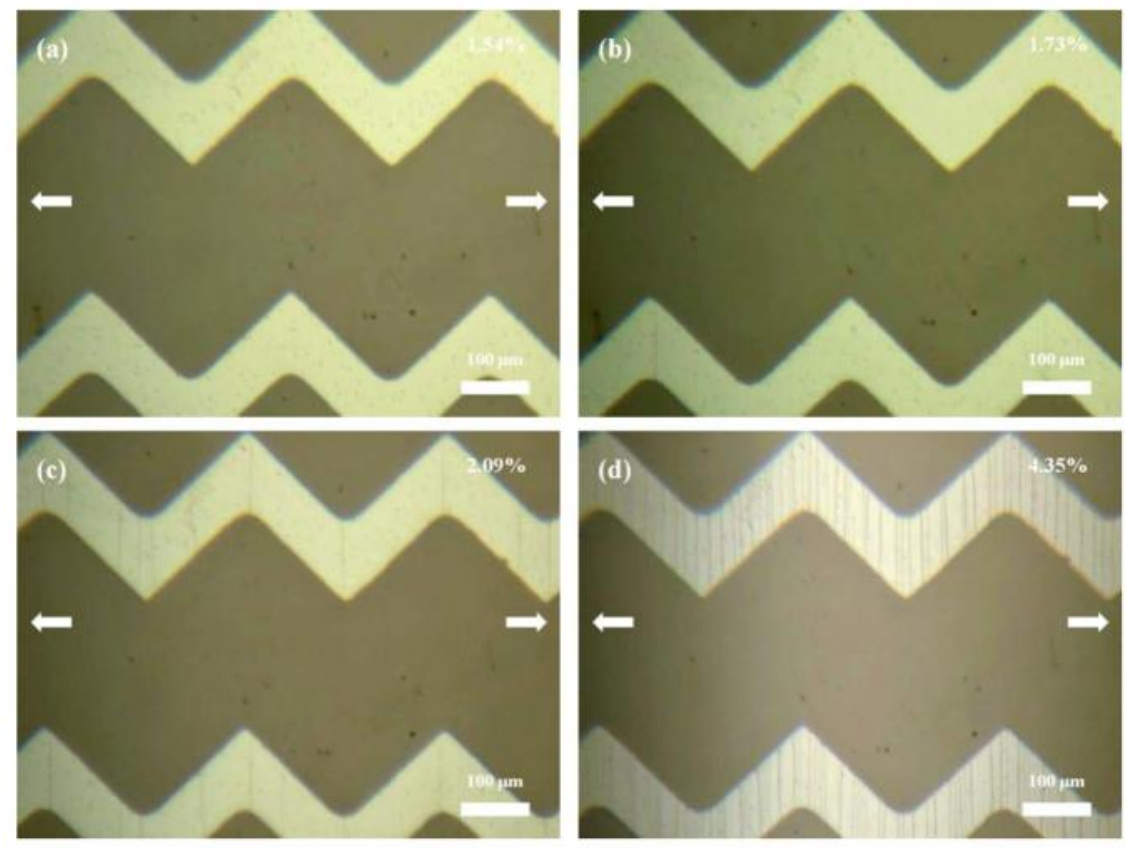

Fig. 4.22 - Optical microscopy images of zigzag patterned ITO-coated PET films under tensile buckling deformation mode showing cracking initiation and propagation up to $6 \%$ strain. Arrows indicate the buckling direction.
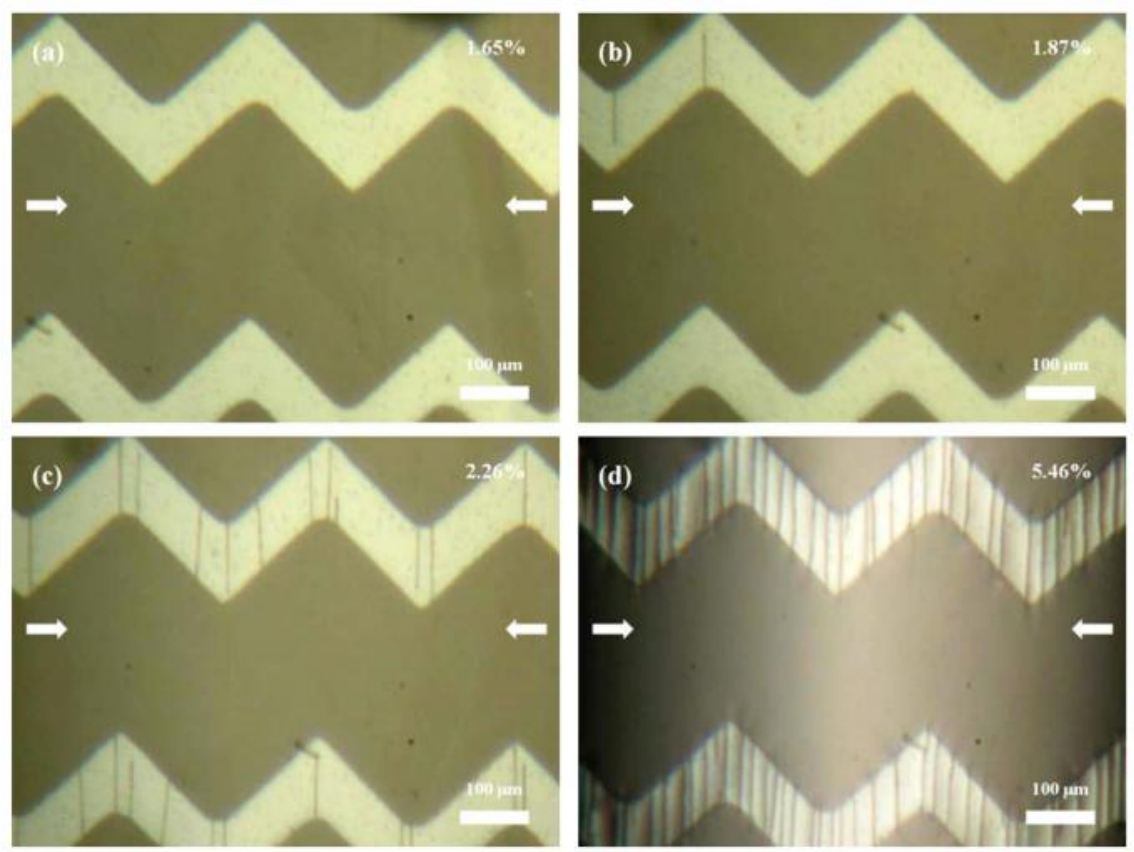

Fig. 4.23 - Optical microscopy images of zigzag patterned ITO-coated PET films under compressive buckling deformation mode showing cracking initiation and propagation up to $6 \%$ strain. Arrows indicate the buckling direction. 
Fig. 4.24 presents SEM micrographs of edge cracking from the circular patterned sample from Fig. 4.24d.

(a)

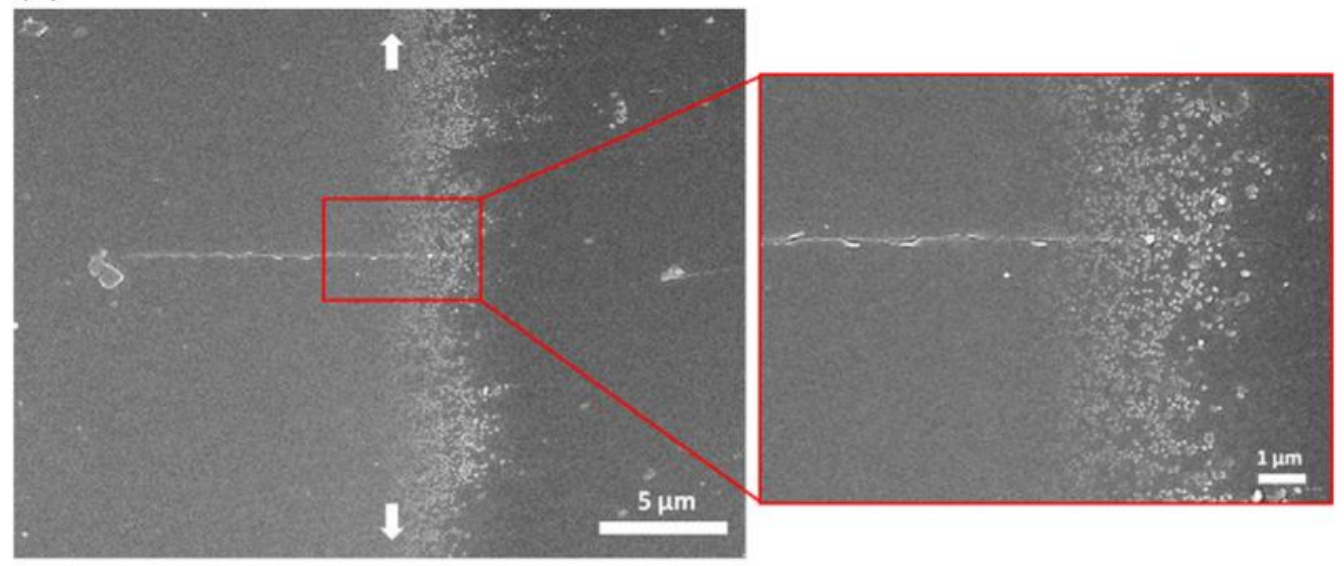

(b)

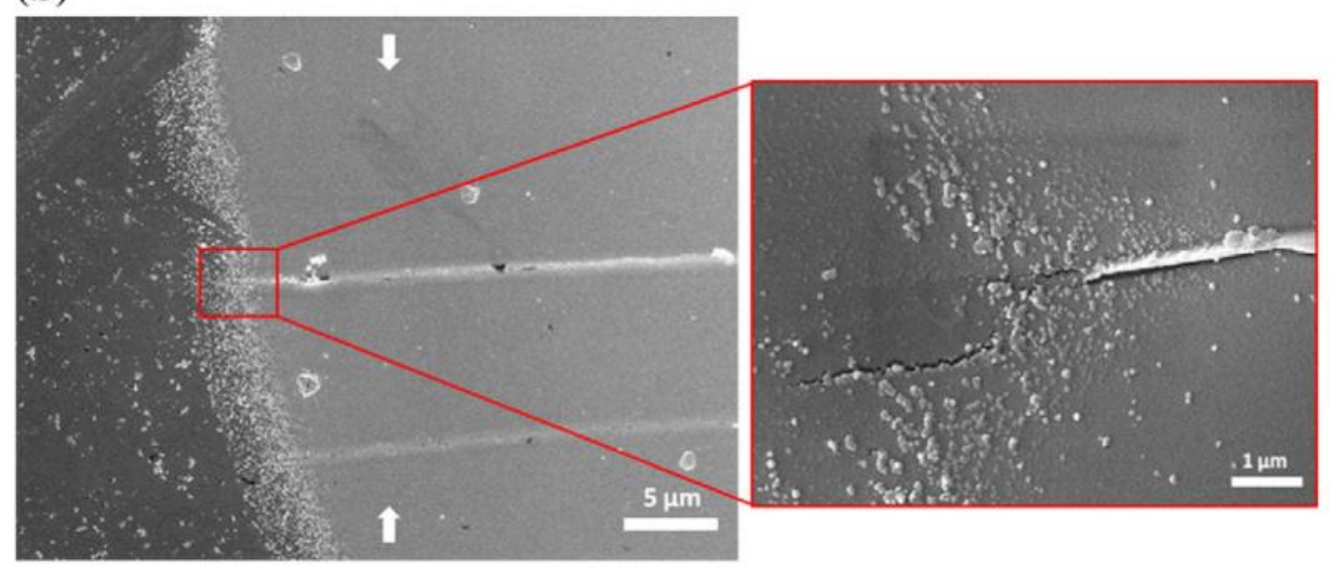

Fig. 4.24 - SEM micrographs of patterned ITO-coated PET films a) under tensile and b) under compressive buckling deformation. Arrows indicate the buckling direction.

This film delamination is attributed to PET substrate contraction underneath the ITO coating, due to the applied compressive buckling strain. Fig.4.24a is an example of cracking propagation seen in samples under tensile buckling strain. For samples loaded in tension and compression, is observed that failure is driven by micro-cracks initiating from the edges. Care must be given when fabricating patterned electrodes to enhance edge fidelity and limit initial cracking for long-term durability of the electrode. 
Fig. 4.25 presents the confirmatory optically observed COS values for controlled buckling experiments. COS measured using this method were significantly higher than those measured electrically. This is due to limitations in the observation technique, where only a small frame of reference of the large sample surface can be observed instantaneously, and due to limitations in human response time.

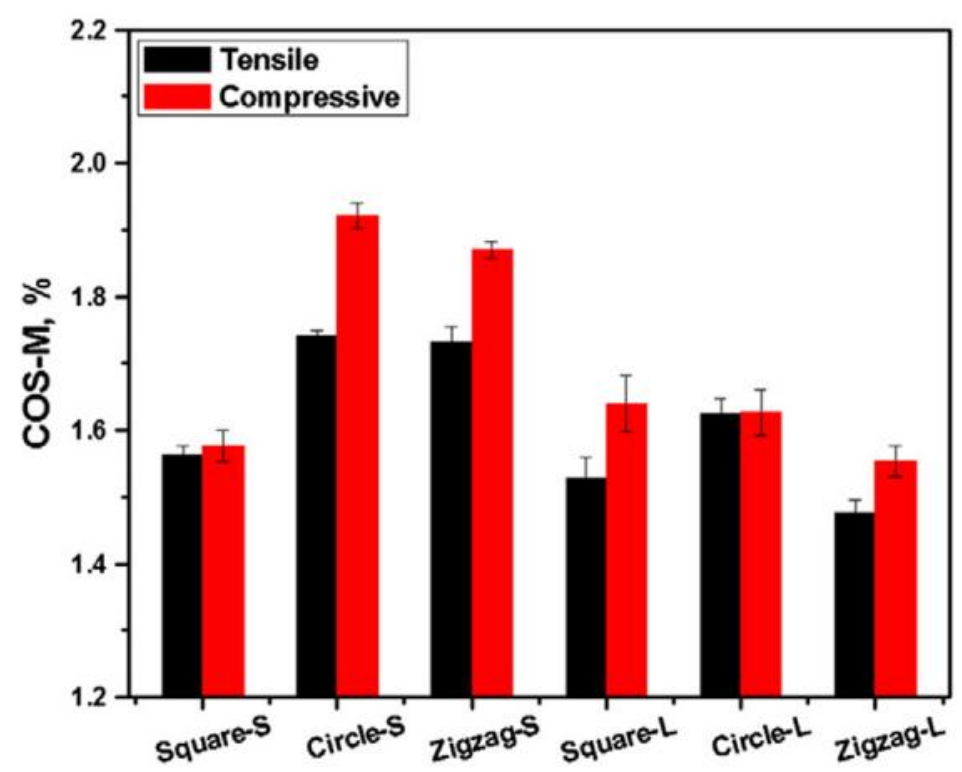

Fig. 4.25 - Crack onset strain from in situ optical microscopy monitoring of ITO-coated PET films. This is supported by Fig. 4.26, which shows the normalized electrical resistance measurements at the optically observed COS values. The previously used metric of a $10 \%$ increase in normalized electrical resistance constituting failure was far exceeded in all cases, particularly in tension samples [88]. 


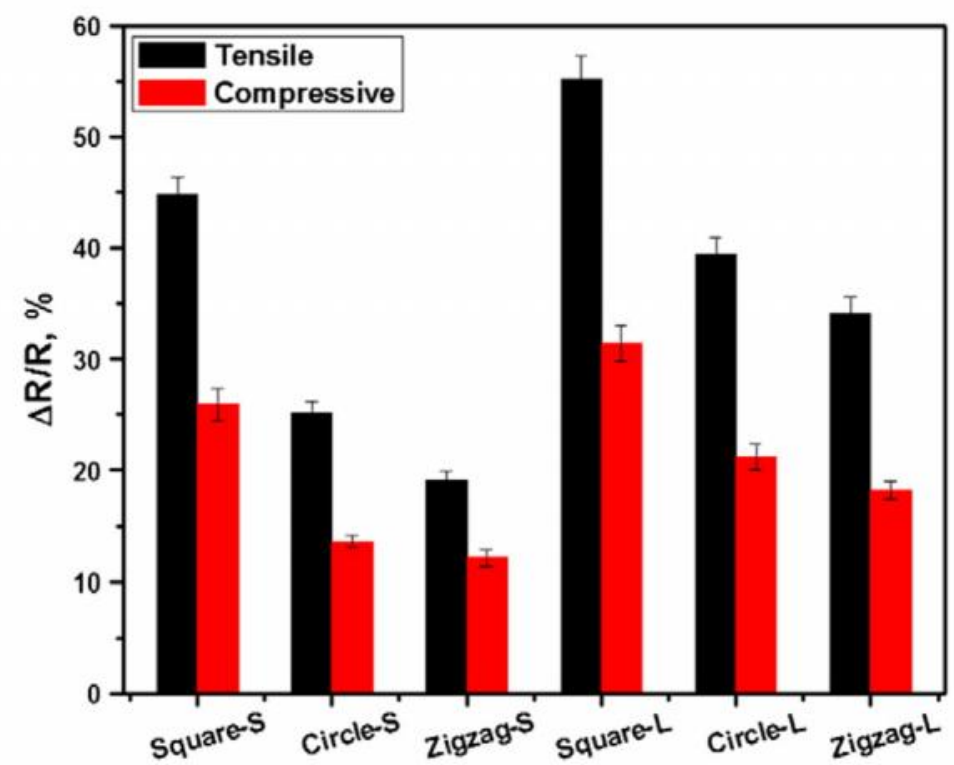

Fig. 4.26 - Normalized electrical resistance at COS measurements for ITO-coated PEN films.

\subsection{Conclusions}

During this work spherical nanoindentation, scratch testing, and controlled film buckling were performed to induce cracking on ITO coatings deposited on polyester substrates in order to further characterize monotonic failure mechanisms associated with the complex system. Spherical indentation results showed that crack initiation occurs at $40 \mathrm{mN}$ normal load or $534 \mathrm{MPa}$ mean contact pressure. At higher loads formation of brittle ring cracking was observed. When loading increased to $200 \mathrm{mN}$, secondary radial cracks formed.

These secondary cracks bridged the primary ring cracks. In addition, it was found that the indent radius imprint on the ITO surface increased linearly with increasing load and it was not depended on coating thickness. Also, it was observed that the ring crack spacing, produced at increasing normal loads, was thickness dependent. 
Scratch testing was also performed in order to assess the coating's cohesive and adhesive failure modes. Two main failure modes were observed. The first was through thickness cracking and the second was buckling spallation. Both mechanisms occurred at some critical load which depended on coating thickness. This dependence was more pronounced in the case of coating spallation. A safe scratch load range for device fabrication for coatings with different thicknesses was suggested.

Controlled buckling experiments under both tensile and compressive mode were performed to characterize the effect of electrode patterned on brittle oxide cracking. In situ monitoring of electrical resistance and optical microscopy were conducted to quantify film failure. Higher crack onset values were observed for the smaller size patterns. Additionally, square-shaped patterns exhibited lower crack onset values due to sharper corners, and therefore higher stress concentration zone. Ex situ characterization indicated cohesive failure and delamination under tensile and compressive buckling condition, especially along the patterned edges. It is believed that better pattern edge definition will improve sustained reliability of electrode films. 


\section{Cyclic tribo-chemical studies of indium tin oxide (ITO) films on polyester substrates}

\subsection{Overview}

This chapter describes investigations of ITO-coated polyester films under cyclic-fatigue loading scenarios. Experiments were designed to examine the effect of combined stresscorrosion cracking and repeated tribological interactions on the durability of ITO electrodes used in flexible devices. Understanding of the sustained functionally of the electrode layer is paramount to an effective device design, as well as manufacture of device components. The work presented in this chapter has been published in Thin Solid Films, doi: 10.1016/j.tsf.2008.10.031. and in Wear, doi: 10.1016/j.wear.2008.12.042.

\subsection{Introduction}

As reported by Gaarenstroom et al. [98] a major limitation in the use of ITO coatings as the anode in devices is the short lifetime due to corrosion. They compared corrosion rates for ITO film electrodes grown using different sputter deposition conditions and they observed that the most corrosion-resistant films exhibited well-formed crystals and showed the most crystallinity. As noted by Raes and Smeets [99] when ITO anodes are exposed to voltage differences in the presence of moisture and contamination, they are also sensitive to corrosion.

Because of the wide application of polycrystalline ITO layers on glass as anodes in optoelectronic devices, there have been a number of studies of ITO corrosion. Most of the work in the literature focuses on halogenated acids, and ITO in contact with 
polyethylenedioxithiophene doped with polystyrene sulfonate in organic light emitting diodes (OLED) devices [140,141]. Scholten and van den Meerakker [142] reported the chemical etching behavior of DC magnetron sputtered ITO films in a large number of acids. They found that the etch rate of crystalline ITO in acids other than the halogenated acids is extremely low. Also, Meerakker et al. [143] observed that ITO films deposited at room temperature are etched by acidic solutions in a matter of seconds whereas crystalline ITO films, deposited at higher temperatures, dissolve in concentrated acids within minutes. It is therefore evident that the ITO etch rate is sensitive to film crystallinity with amorphous ITO being etchable with dilute acids including acetic acid [94]. The etching of ITO electrodes, used in OLED's, by residual acid in a solid conducting polymer layer has also been reported [141,144]. For flexible electronic devices, acrylate-based inks and adhesives can be widely used and residual acrylic acid from these polymer layers can cause premature failure of amorphous ITO films on flexible substrates.

In addition to being in contact with acrylic acid, the ITO anode may also be under the influence of an externally applied mechanical stress, which is needed in order to bend the flexible device. Little research has been done to date on this issue. The combination of mechanical stress and chemical corrosion can lead to stress-corrosion cracking. It is important in any flexible optoelectronic device design to understand the degradation of properties due to all three of these phenomena.

Additionally, little research has been done to date on the macroscale tribological behavior of ITO coatings, deposited on polyester substrates. Shin et al. [141] found that the friction force for ITO on PET substrate as measured by the scratch adhesion test as a function of 
DC sputtering power, increases almost linearly. However, the friction force for ITO on glass and acrylic seems to increase slightly with increasing DC power. It is observed that the friction force of as-deposited ITO films grown on PET and acrylic substrates is always greater than that on glass.

Furthermore, Cairns et al. [145] investigated the wear of a sputtered ITO layer on a PET substrate used as a top-sheet in a touchscreen configuration, where a stylus was drawn repetitively on the PET surface for up to 60,000 sliding cycles. They reported transfer of ITO flakes from the top-sheet to the bottom ITO surface, often called adhesive wear. They also observed ITO pitting and polymer extrusion through the ITO pits. At a higher number of cycles they also observed ITO cracking. Additionally, the adhesive and wear properties of ITO sputtered glass substrates were investigated by Kim et al. with a sapphire ball was slid repeatedly on an ITO film flat and the electrical resistance of the ITO surface monitored in situ [146].

The choice of ITO flat sliding against another ITO flat surface was of both fundamental and practical interest. This was because there is little previous work on the tribological interaction between ITO surfaces. Also, the shear interactions between such sliding surfaces are of particular interest. In practical terms, it is important to compare the dry and wet macro-scale wear characteristics of ITO films, since manufacturing of flexible devices will most certainly involve roll-to-roll processing, which in turn may introduce complex stress states and repetitive ITO surface contact under moderate loads, in both dry and wet acidic environments. Also, it is expected that these flexible energy harvesting devices, in particular, will be needed to adequately function in harsh environments such as in automotive applications and space applications where mechanical and macro-scale 
tribological properties become a critical reliability parameter. Finally, it is important to note that repetitive flexing of the device may cause internal shear interactions and limited sliding, which may lead to functional failure, between the different layers in the presence of acidic containing layers.

\subsection{Experimental Methods}

\subsubsection{Materials}

ITO was deposited on PET substrates (DuPont ST504) by DC magnetron sputtering at room temperature, and the samples had a sheet resistance of $100 \Omega / \mathrm{sq}$. The sputtering power was approximately $1 \mathrm{~kW}$. Argon was used as the processing gas, along with a small quantity of oxygen. The oxygen partial pressure was approximately $2 \mathrm{mPa}$. Film deposition rate was between 4 and $5 \mathrm{~nm} / \mathrm{min}$. The chemical composition of the ITO target was $\operatorname{In}_{2} \mathrm{O}_{3}: \mathrm{SnO}_{2}=90: 10$.

The thickness of the ITO coating ( $100 \Omega / \mathrm{sq}$. sheet resistance) was equal to $200 \mathrm{~nm}$, measured by stylus profilometry (Veeco Dektak 150); and therefore, its specific electrical resistance, $\rho$, is equal to $2 \times 10^{-3} \Omega \mathrm{cm}$. The thickness of the ITO coating (200 $\Omega / \mathrm{sq}$. sheet resistance), which was used during mechanical testing for comparison purposes, was measured to be equal to $100 \mathrm{~nm}$ using a stylus profilometer (Veeco Dektak 150). The specific electrical resistance is the same as in the previous case (i.e. $2 \times 10^{-3} \Omega \mathrm{cm}$ ).

The material used in the tribological study, for both top and bottom surfaces, was also ITO-coated PET. The ITO is sputtered, at room temperature, on the polyester (Melinex ST504, $125 \mu \mathrm{m}$ thick) surface that was heat treated and biaxially oriented. The ITO surfaces that slide against each other had an electrical resistivity of $200 \Omega / \mathrm{sq}$. The 
thickness of the ITO layer, as measured by tapping-mode AFM, was $100 \pm 20 \mathrm{~nm}$ and its RMS roughness was measured to be around $5.5 \mathrm{~nm}$.

\subsubsection{Tensile Testing}

ITO-coated PET samples, $200 \Omega$ /sq, were tested in uniaxial tension with in situ electrical resistance monitoring using a commercial mechanical testing machine (Instron 4410). Typical 'dog-bone' specimens with gauge length of $25 \mathrm{~mm}$ and width of $5 \mathrm{~mm}$ were used and the operating crosshead speed was $0.3 \mathrm{~mm} / \mathrm{min}$. ITO electrical resistance data were recorded during the tests using an Agilent 349708 data acquisition/switch unit. In addition, corrosion measurements were conducted, at room temperature, with ITO-coated PET strips $(6 \mathrm{~cm}$ in length and $1 \mathrm{~cm}$ in width) being immersed in acrylic acid solutions of 0.1 M concentration. The ITO two-point electrical resistance was measured in situ using an Agilent 349708 data acquisition/switch unit.

\subsubsection{Electrochemical Characterization}

Electrochemical measurements were performed on ITO-coated PET samples with resistivity equal to $100 \Omega /$ sq. The samples were washed with distilled water and dried before performing the electrochemical measurements. Platinum gauze was used as the counter electrode and a saturated $\mathrm{Ag} / \mathrm{AgCl}$ electrode was employed as the reference

electrode. The ITO sample area exposed was $1 \mathrm{~cm}^{2}$. Electrochemical impedance spectroscopy (EIS) measurements were conducted using an impedance analyzer (Solartron Analytical, 1260) connected to a potentiostat (Solartron Analytical, 1287). A stable open-circuit potential was attained before performing the EIS measurement. EIS

spectra were recorded over a frequency range of $10^{6}$ to $0.01 \mathrm{~Hz}$ with applied AC 
amplitude of $10 \mathrm{mV}$.

\subsubsection{Time-of-Flight Secondary Ion Mass Spectrometry (TOF-SIMS)}

High-resolution TOF-SIMS analysis was performed with a TFS series instrument

(Charles Evans \& Associates, Redwood City, CA), using a $69 \mathrm{Ga}^{+}(15 \mathrm{keV})$ liquid metal ion source. Test coupons were prepared with a $9.5 \mathrm{~cm} \times 2 \mathrm{~cm}$ strip of pressures sensitive adhesive (PSA) adhered to the center of a $9.5 \mathrm{~cm} \times 9 \mathrm{~cm}$ ITO coated rectangle of PET. SIMS depth profiling was performed through the PSA and ITO layers. The stack-up is PSA/PET/PSA/ITO/SiO2/PET.

\subsubsection{Stress Corrosion Cracking Measurements}

Mandrels made from chlorinated polyvinyl chloride (c-PVC) of various diameters were utilized to apply various bending strains to sample strips of ITO-coated PET. The sample strips were adhered around the center of the mandrels using double-sided adhesive tape.

Small strips of aluminum foil, approximately a centimeter in width, were wrapped around each end of the sample where leads, for electrical resistance monitoring, are to be attached. This electrically-transmissive buffer between the applied lead and the ITO surface allowed for a better electrical connection and minimal damage to the ITO conductive surface. The leads were connected to a digital switchbox that allowed for multi-channel data logging (Agilent 349708 Data Acquisition/ Switch Unit) and were then attached to opposite sides of the ITO coated PET strip. The wrapped mandrel was then dipped into various $50 \mathrm{ml}$ solutions of acrylic acid of varying concentration. Data were recorded every 10 min using Agilent BenchLink software. Tested surfaces were 
investigated using a Hitachi S-4000 scanning electron microscope (SEM) with a field emission gun. The operating voltage was $20 \mathrm{kV}$. Samples were coated with a $10 \mathrm{~nm}$ gold layer, prior to SEM investigation, using a Hummer X (Anatech LTD) sputter coater.

\subsubsection{Wear Testing}

The bottom counterface sample, $36 \mathrm{~cm}^{2}$ area, was adhered to a glass base substrate using a double-sided adhesive tape. Particular care has to be taken when adhering the sample to the glass substrate in order to avoid premature cracking of the brittle ITO coating. The sample fit inside a plastic container. Under wet sliding conditions, the container was

filled with acrylic acid of $0.1 \mathrm{M}$ concentration. The top ITO-coated PET surface, $12 \mathrm{~cm}^{2}$ area, slid in a reciprocating fashion against the bottom surface. The top sample was adhered to the top metal surface using double-sided adhesive tape. The electrical resistance of the top ITO surface was measured before and after testing. A constant normal load equal to $3.5 \mathrm{~N}$ was applied. Typical flexible optoelectronic devices are expected to have a display area ranging from 10 to $20 \mathrm{~cm}^{2}$ and $3.5 \mathrm{~N}$ is a typical load under moderate handling conditions. At each end of the bottom conductive surface a metal bar was placed in order to act as an electrical contact for in situ electrical resistance, $R$, monitoring of the bottom ITO surface. Electrical resistance data were recorded during testing using an Agilent 349708 data acquisition/switch unit. Each test was conducted using a fixed reciprocating speed of 34 cycles per second. Wear tests, in dry and wet conditions, were run for up to a few thousand reciprocating cycles. Each reciprocating cycle is equal to $50.8 \mathrm{~mm}$ of linear sliding distance. Two samples were tested at each set of conditions. 
Weight measurements were conducted before and after each test for both the top and bottom ITO surfaces using a digital balance (DV215CD, Ohaus, $0.01 \pm 0.02 \mathrm{mg}$ ). After testing and before weighing the samples were washed in distilled water and dried using compressed air.

\subsubsection{Microscopy}

Virgin ITO-coated PET surfaces were studied using a Molecular Imaging Pico SPM II AFM microscope in tapping mode. Worn surfaces were studied using a Leica optical microscope, magnifications 10, 20 and 50 times, equipped with a frame grabber (Guppy, Allied Visions Technology). Also, worn surfaces were observed using a Hitachi S-4000 scanning electron microscope with a field emission gun. Samples were sputtered with a $10 \mathrm{~nm}$ gold coating (Hummer $\times$ sputtering machine) prior to scanning electron microscopy (SEM) observation in order to increase their conductivity. Finally, worn surface topography was recorded using a Veeco Dektak 110 surface stylus profilometer with a 3D option. The applied load of the stylus was $1 \mathrm{mg}$ and the stylus radius was equal to $12.5 \mu \mathrm{m}$.

\subsection{Results and Discussion}

\subsection{Corrosion Studies}

Corrosion characterization of ITO-coated PET strips was undertaken in order to measure the critical time at which the ITO electrical resistance starts to increase. Similarly to the previously discussed mechanical failure under tensile and compressive strains, this increase was correlated with corrosion and etching of the ITO surface when immersed in 
$0.1 \mathrm{M}$ acrylic acid solution. It is important to note that the $0.1 \mathrm{M}$ concentration was selected from a range of different acid concentrations $(0.1-0.9 \mathrm{M})$ because the time to corrode ITO surface areas is sufficient to allow meaningful observations.

As shown in Fig. 5.1, the ITO two-point electrical resistance is nearly stable for around $40 \mathrm{~min}$.

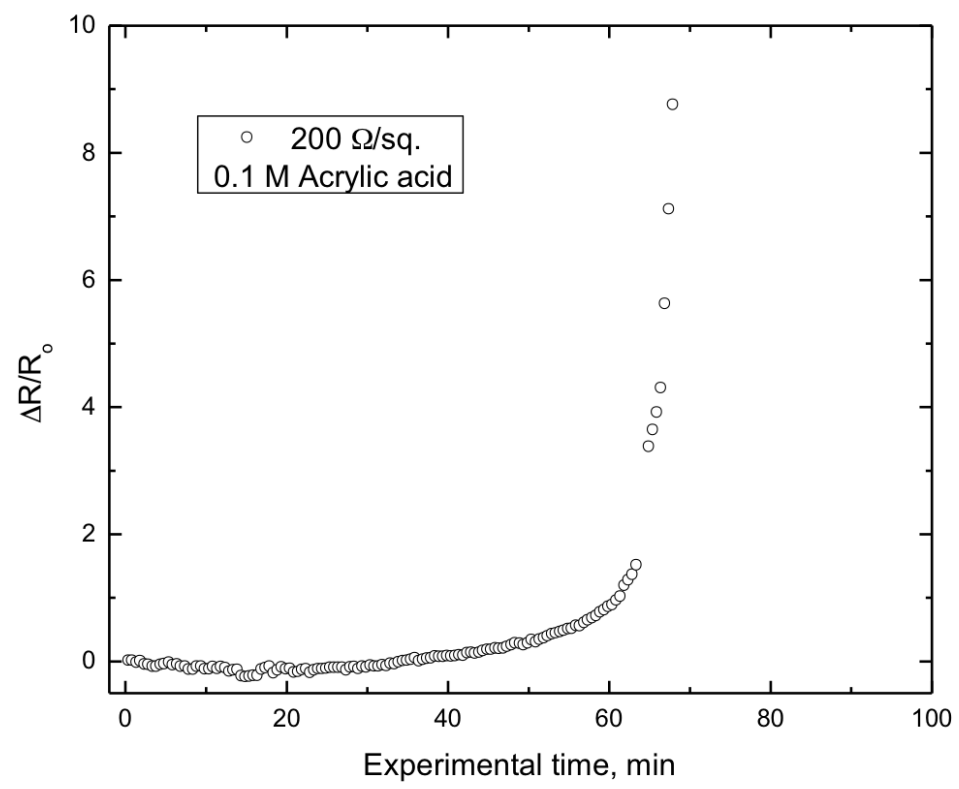

Fig. 5.1 - Electrical resistance versus time for a $200 \Omega / \mathrm{sq}$, ITO-coated PET sample immersed in acrylic acid of $0.1 \mathrm{M}$ concentration.

Then, for the next $20 \mathrm{~min}$ it starts slowly to increase until it reaches a stage at which the increase is abrupt. The latter indicates irreversible damage due to corrosion of the ITO coating. Microscopy reveals total etching of large ITO surface areas. It has been also observed that corrosion forms around the roughest ITO areas.

\subsubsection{Depth Profiling of SIMS}

A test coupon of ITO-coated PET in contact with a PSA was aged for 10 days at $60{ }^{\circ} \mathrm{C}$ 90\% RH. The acrylic acid content increased by a factor of eight during ageing. An aged 
and an un-aged sample were characterized using depth-profiling TOF-SIMs. Secondary ion counts as a function of sputtering time (proportional to depth) for aged and un-aged samples are shown in Fig. 5.2. The un-aged coupon exhibits a sharp increase in secondary ions of indium 113 and indium 115 at the PSA/ITO interface. In the aged sample, the interface is much less sharp with a significant amount of indium in the PSA. This leeching of indium into the PSA is consistent with an etching process and has been reported previously by Bijnens et al. [147] in their study on the degradation of OLEDs with conductive-polymer coated ITO electrodes. The SIMS data confirm that the ITO interacts with an acrylate PSA, with interdiffusion of indium into the PSA layer.
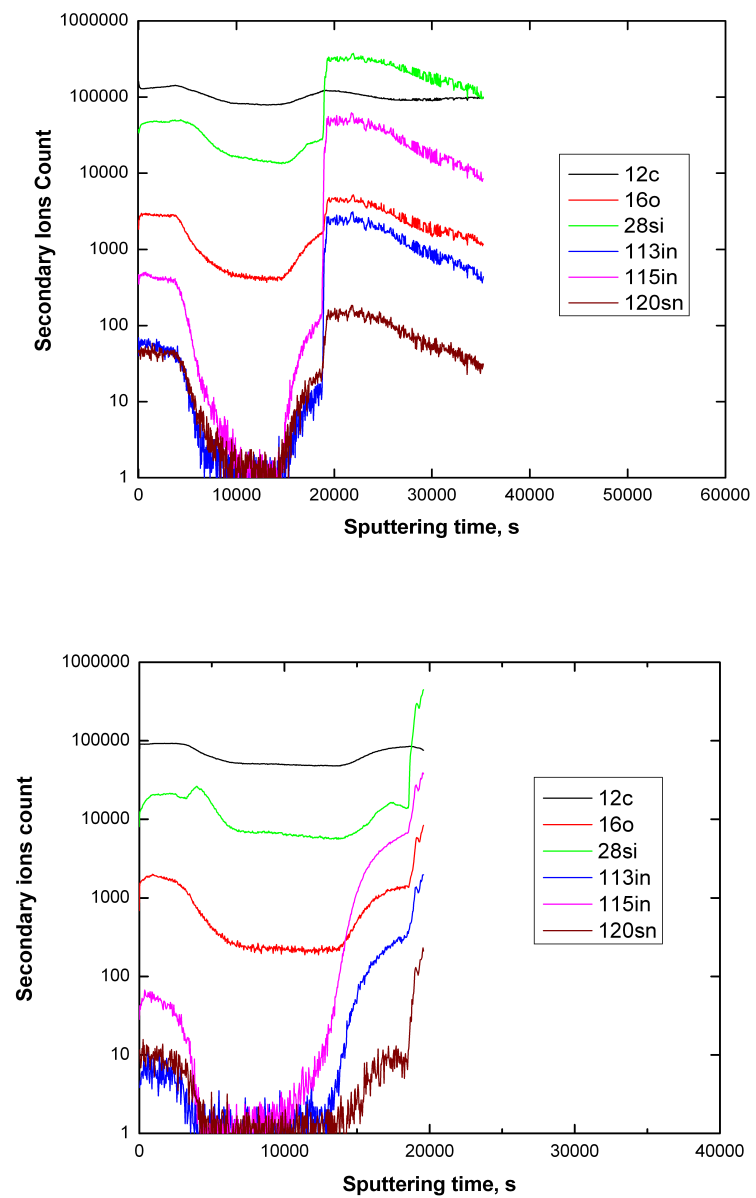

Fig. 5.2 - a) TOF-SIMS depth profile of an un-aged test coupon of PSA on ITO and b) TOF-SIMS depth profile of an aged coupon of PSA on ITO. The sample was aged at $60{ }^{\circ} \mathrm{C}$. 


\subsubsection{Electrochemical Impedance}

Fig. 5.3 shows the variation of open-circuit potential of ITO with time in $0.1 \mathrm{M}$ acrylic acid. A stable open-circuit potential is attained after a time lapse of about $20 \mathrm{~min}$.

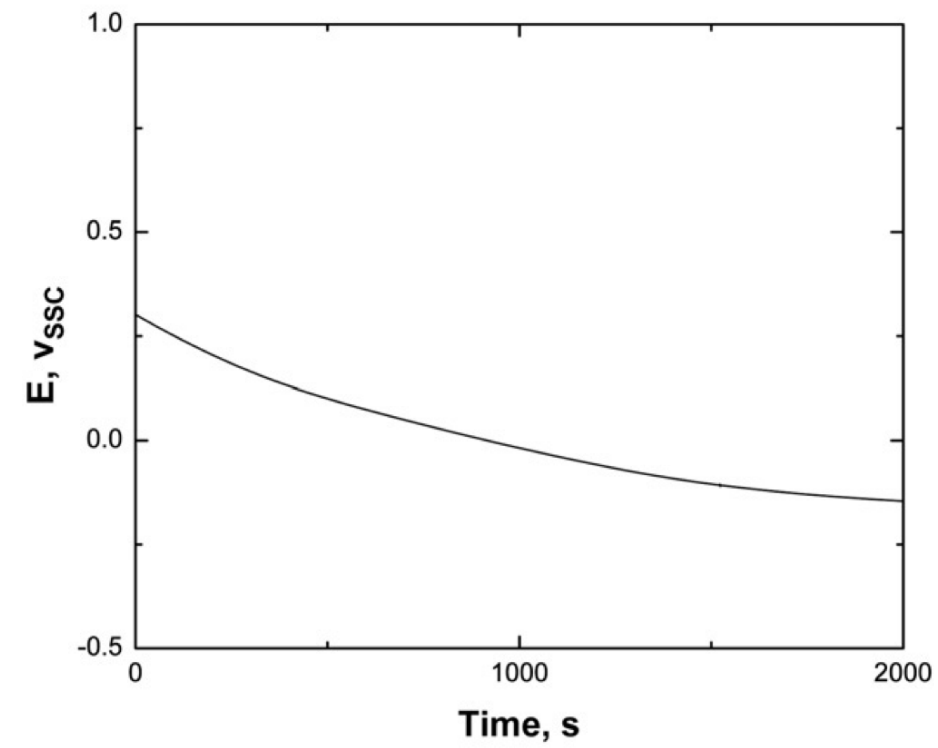

Fig. 5.3 - Open-circuit potential versus time curve for an ITO film in $0.1 \mathrm{M}$ acrylic acid.

Typical EIS spectra obtained for ITO coated polymer substrate immersed in $0.1 \mathrm{M}$ acrylic acid are shown in Fig. 5.4.

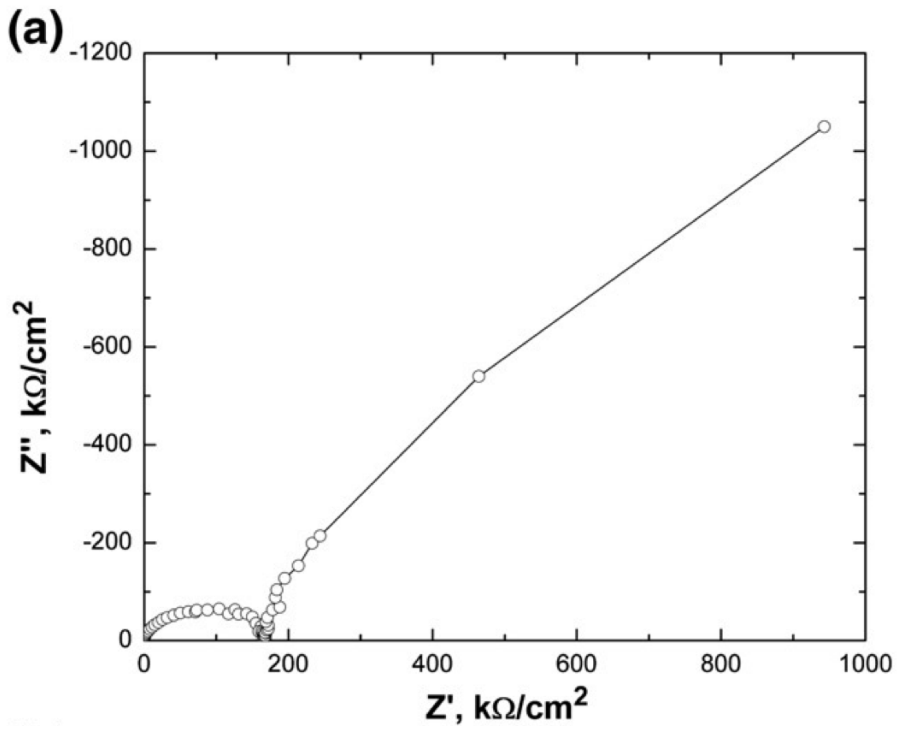




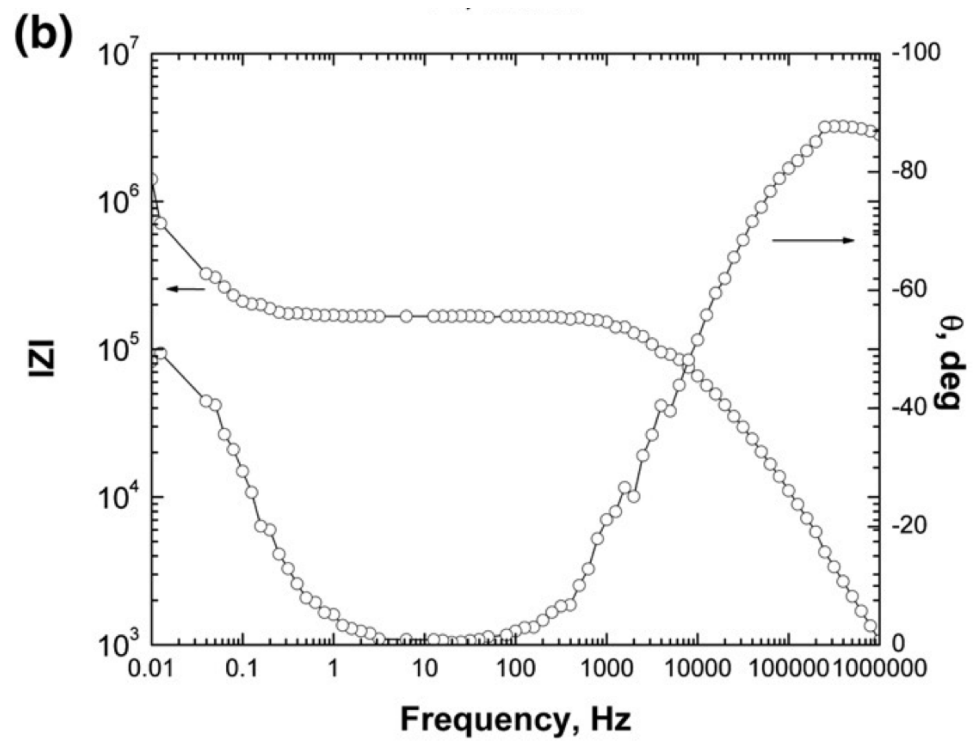

Fig. 5.4 - Electrochemical impedance specra for ITO in 0.1M acrylic acid: a) Nyquist plot and b) Bode (frequency and phase angle) plots.

Randle's equivalent circuit model with Warburg impedance was employed to analyze the experimental impedance data. The Warburg impedance accounts for the diffusion of ions from the ITO electrode surface to the electrolyte. This circuit includes ohmic resistance of the electrolyte solution, $\mathrm{R}_{\mathrm{S}}$, double-layer capacitance, $\mathrm{Cdl}$, electron-transfer resistance, $\mathrm{R}_{\mathrm{ct}}$, and Warburg impedance, $\mathrm{W}$. The parameters obtained by fitting the experimental data to the equivalent circuit elements are as follows: $\mathrm{R}_{\mathrm{S}}=61.8 \Omega, \mathrm{Cdl}_{\mathrm{dl}}=0.1 \mathrm{nF}, \mathrm{R}_{\mathrm{ct}}=0.1$ $\mathrm{m} \Omega$, and Warburg: W1- R=9.1 $\times 10^{5}$, W1-T=27.4, W1-P=0.7

\subsubsection{Stress Corrosion Cracking}

We have focused in this work on $100 \Omega /$ sq ITO coated PET samples. In order to optimize the acid concentration we immersed un-deformed samples in acrylic acid concentrations ranging from 0.1 to $0.9 \mathrm{M}$ and we monitored the change in electrical resistance in situ. The graph shown in Fig. 5.5 summarizes the findings. 


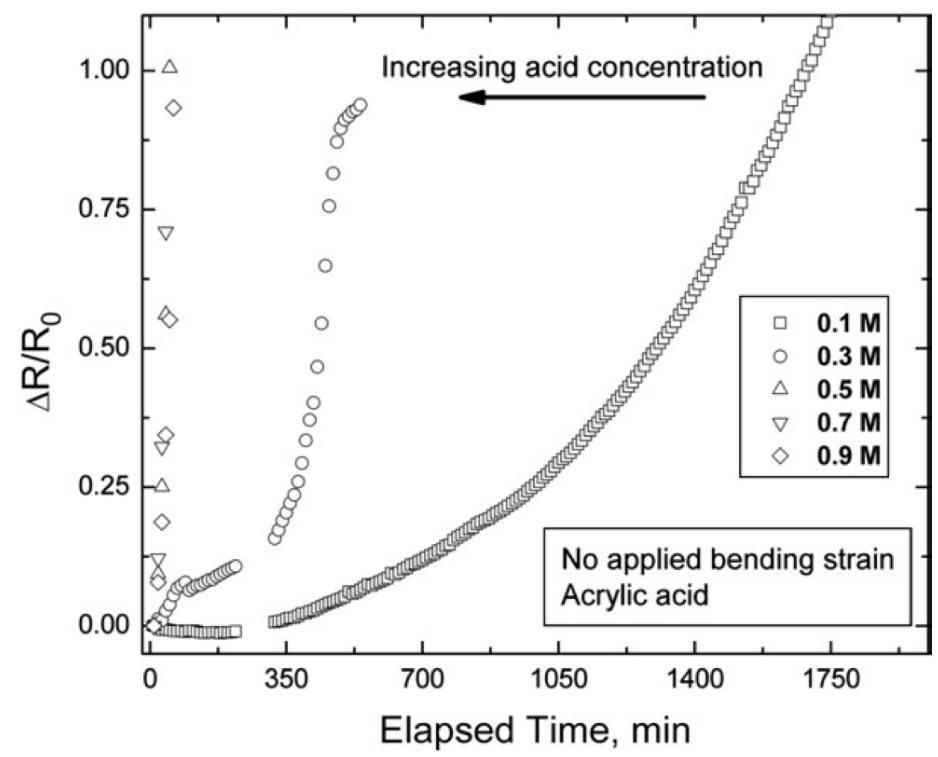

Fig. 5.5 - Normalized electrical resistance versus experimental time for unstrained ITO-coated PET immersed in acrylic acid of various concentrations.

As expected, the slowest increase in ITO electrical resistance took place for the sample that was immersed in $0.1 \mathrm{M}$ acrylic acid. The normalized electrical resistance for this particular sample is constant and close to zero and then at around $350 \mathrm{~min}$ of experimental testing an increase in resistance is observed. For the $0.3 \mathrm{M}$ sample, the resistance increase is more sudden, indicating faster corrosion of the ITO layer as compared with $0.1 \mathrm{M}$. Higher acid concentrations such as $0.5,0.7$ and $0.9 \mathrm{M}$ promote fast corrosion of the ITO coating. Stress-corrosion cracking becomes particularly important for low acid concentrations where corrosion alone does not dominate.

By studying the SEM images of ITO surfaces immersed in $0.3 \mathrm{M}$ acrylic acid, as shown in Fig. 5.6, a corrosion-dominated ITO cracking process is revealed. 


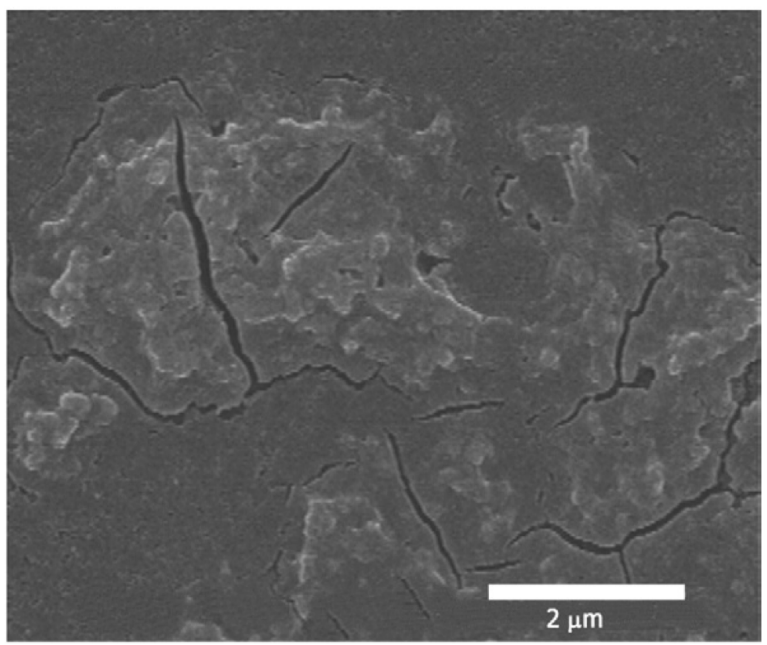

Fig. 5.6 - SEM image showing stress-corrosion cracking formation in an ITO-coated PET, $100 \Omega / \mathrm{sq}$, immersed in $0.3 \mathrm{M}$ acrylic acid when an external bending strain of $0.96 \%$ is applied.

Corrosion cracks, similar in morphology to cracks caused by externally applied biaxial strains, are evident for the $0.96 \%$ bending strain sample. Branching cracks are observed to form crack networks in rough areas of the sample. The strain of $0.96 \%$ is significantly lower than the $1.75 \%$ tensile strain needed to cause cracks in the absence of acrylic acid. It is therefore evident that surface defects and the presence of acid can have a significant deleterious impact on the ITO film.

In addition, ITO coated samples of $100 \Omega /$ sq were wrapped around different diameter mandrels generating various externally applied bending strains and they were then immersed in $0.1 \mathrm{M}$ acrylic acid. Fig. 5.7 shows the results of this experiment. Fig. 5.7a shows the electromechanical behavior of the composite system. 

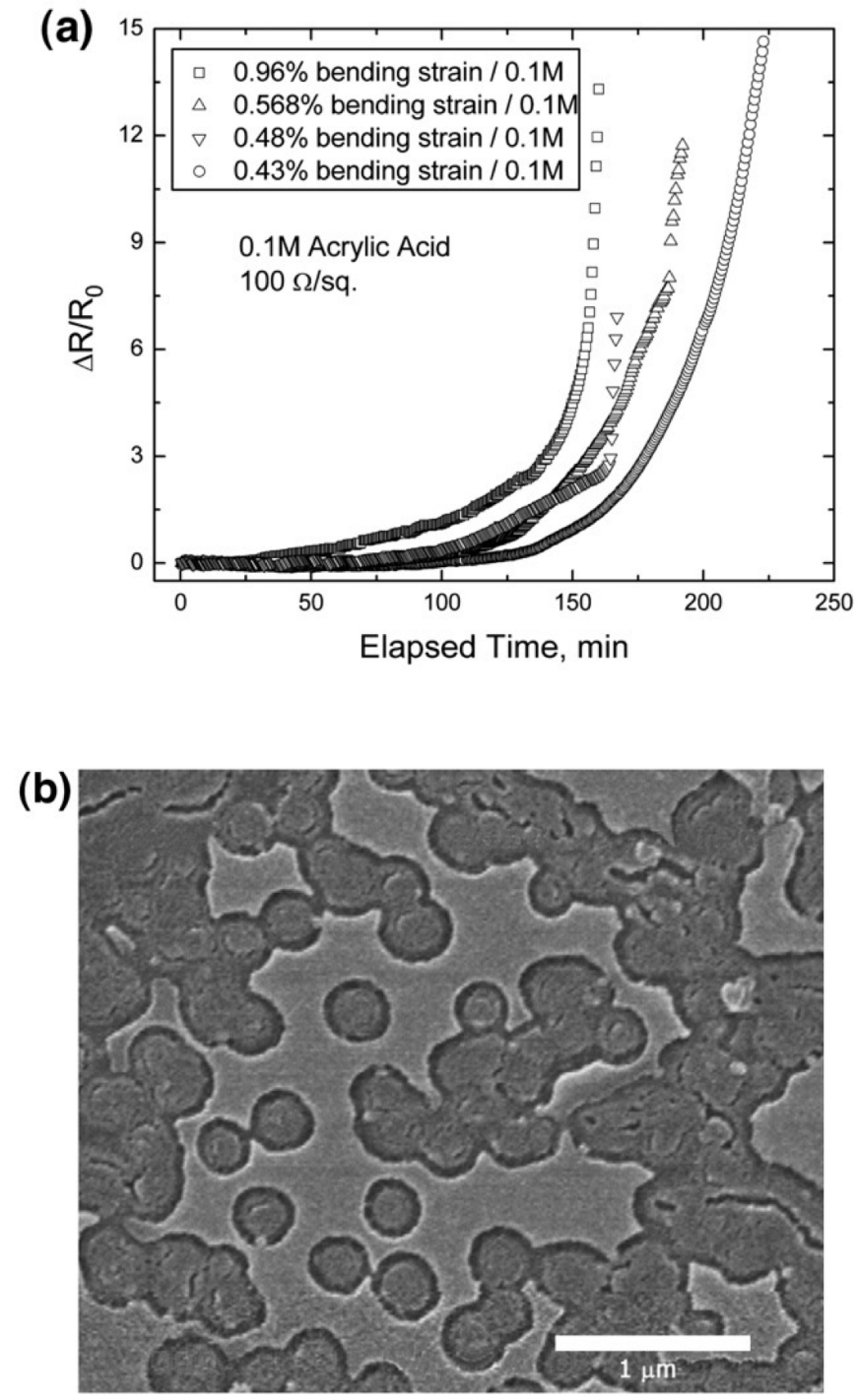

Fig 5.7 - a) Electromechanical behavior of ITO-coated PET samples immersed in $0.1 \mathrm{M}$ acrylic acid for various applied bending strains and b) SEM image showing a region of an ITO-coated PET, $100 \Omega / \mathrm{s}$, strained at $0.78 \%$ in $0.1 \mathrm{M}$ acrylic acid.

It is evident in the graph, that the higher the applied strain the earlier the electrical

resistance starts to increase. Therefore, the contribution of externally applied mechanical strain is considerable. The combined action of mechanical stress and corrosion promotes cracking at stresses less than a quarter of those needed for electrical failure in a noncorrosive environment. It is also important to note that the time to failure in a corrosive environment, under approximately $1 \%$ tensile strain, can reduce the total time to failure 
by as much as a third as compared with the case without applied mechanical stress shown in Fig. 5.1. SEM imaging, Fig. 5.7b, reveals corrosion-driven ITO failure and mechanical cracking. The polymer substrate is clearly visible, in several regions. The combined effect of applied stress and corrosion must therefore be taken into account in any prediction of lifetime in the field.

\subsubsection{Wear Testing}

During both dry and wet wear testing, the two-point electrical resistance of the bottom ITO counterface is monitored throughout. This is an advantage when considering conductive coatings since it can give an insight of the wear mechanisms involved. Fig. 5.8 presents the variation of the ITO normalized resistance against the number of reciprocating dry wear cycles.

The scale of the ordinate axis is relatively large in order to cover all resistance changes. At 100 cycles a $40 \%$ resistance increase is observed. Above 1000 cycles the resistance increases in a more noisy fashion until an abrupt increase, which is observed to occur above the 10,000 cycles threshold. Changes in electrical resistance can be correlated with changes in the ITO surface due to friction and wear. In particular, noisy ITO resistance changes may indicate cracking, layer delamination and abrasion mechanisms active between the two surfaces. Above 10,000 reciprocating cycles, the abrupt increase in ITO electrical resistance shows significant loss of coating functionality.

The variation of the bottom ITO electrical resistance was also monitored in wet sliding conditions and more specifically in the presence of $0.1 \mathrm{M}$ acrylic acid, as shown in Fig. 5.9 . 


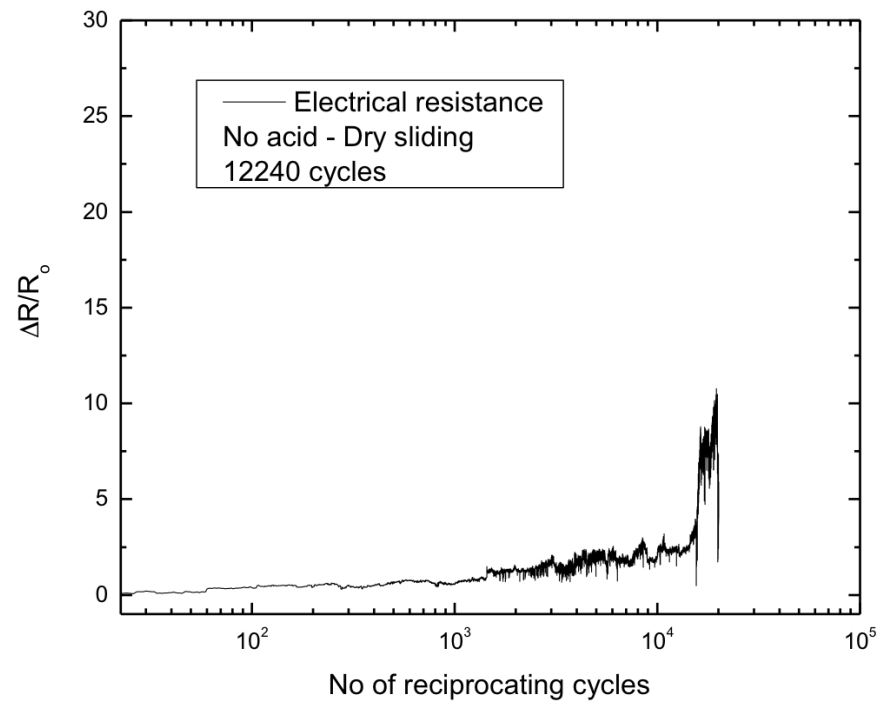

Fig. 5.8 - Normalized electrical resistance versus number of reciprocating cycles (semi-log plot) in dry conditions.

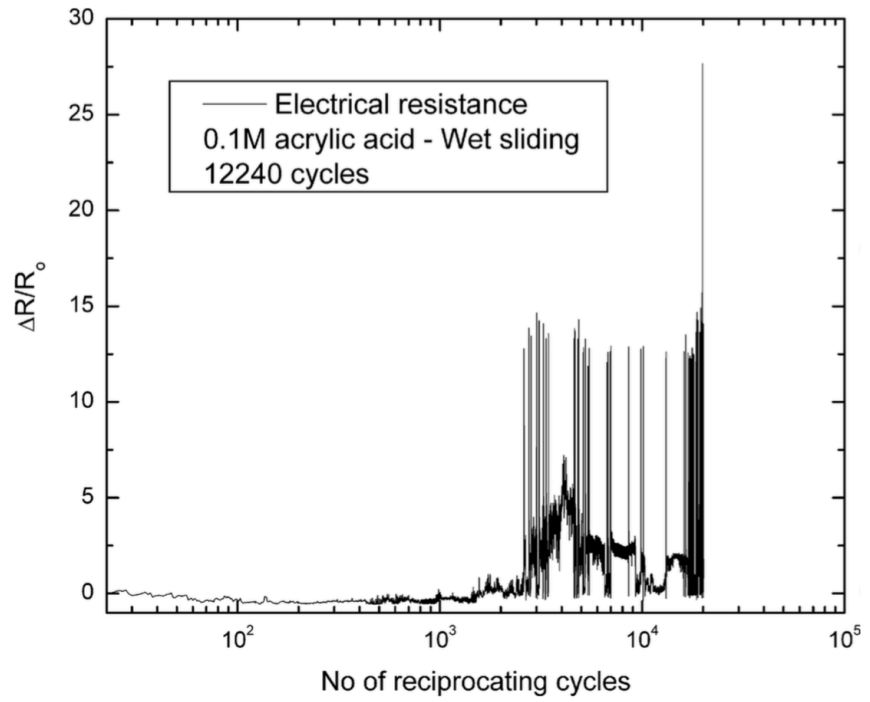

Fig. 5.9 - Normalized electrical resistance versus number of reciprocating cycles (semi-log plot) in $0.1 \mathrm{M}$ acrylic acid.

The changes in electrical resistance are observed to be of a different trend as compared to the ones observed in dry sliding conditions. It is observed that during the first 100 cycles the resistance remains mostly constant and no significant increase is observed. This is surprising but can be probably attributed to the presence of the acidic solution. We 
speculate that the solution forms a protective low friction film, on the interface between the ITO surfaces, providing low friction and reducing the damage caused due to wear and other failure mechanisms. At around 3000 reciprocating cycles, the resistance is observed to abruptly increase in a noisy fashion. This may indicate a breakage of the cohesion of the protective acidic film that forms between the two sliding surfaces. It is also possible that the top conductive surface is bridging the bottom counter-face, which results in a noisy resistance variation. Between 4000 and 10,000 cycles a significant drop in resistance is measured. It is speculated that the bridging effect provided by the topreciprocating surface is still strong and that ITO particle transfer, and subsequent entrapment, from the top to the bottom surface may take place. Above 10,000 reciprocating cycles the resistance is observed to increase again with the noisy trend still present.

Generally, it can be assumed that since there is resistance increase in both dry and wet sliding conditions, the tensile stresses present in the bottom ITO surfaces exceed the $2.25 \%$ threshold strain measured by tensile electromechanical testing. It is therefore probable to expect cracking and adhesive failure of the bottom ITO coating. On the other hand, for the wet sliding case only, it is not straightforward to assume that the electrical resistance of the bottom surface will abruptly increase after 60 min (i.e. 2040 reciprocating cycles) based only on the corrosive action of the acidic solution. This is because of the complex contact of two conductive surfaces in an acidic medium as opposed to pure acid-surface interaction in the case of straight corrosion (Fig. 5.1).

In order to form a better understanding of the wear processes involved, the two-point electrical resistance of the top- reciprocating surface was measured before and after 
testing for both wet and dry sliding conditions, against the number of reciprocating cycles. Fig. 5.10 shows the percentage increase in electrical resistance against number of reciprocating cycles for both wet and dry sliding.

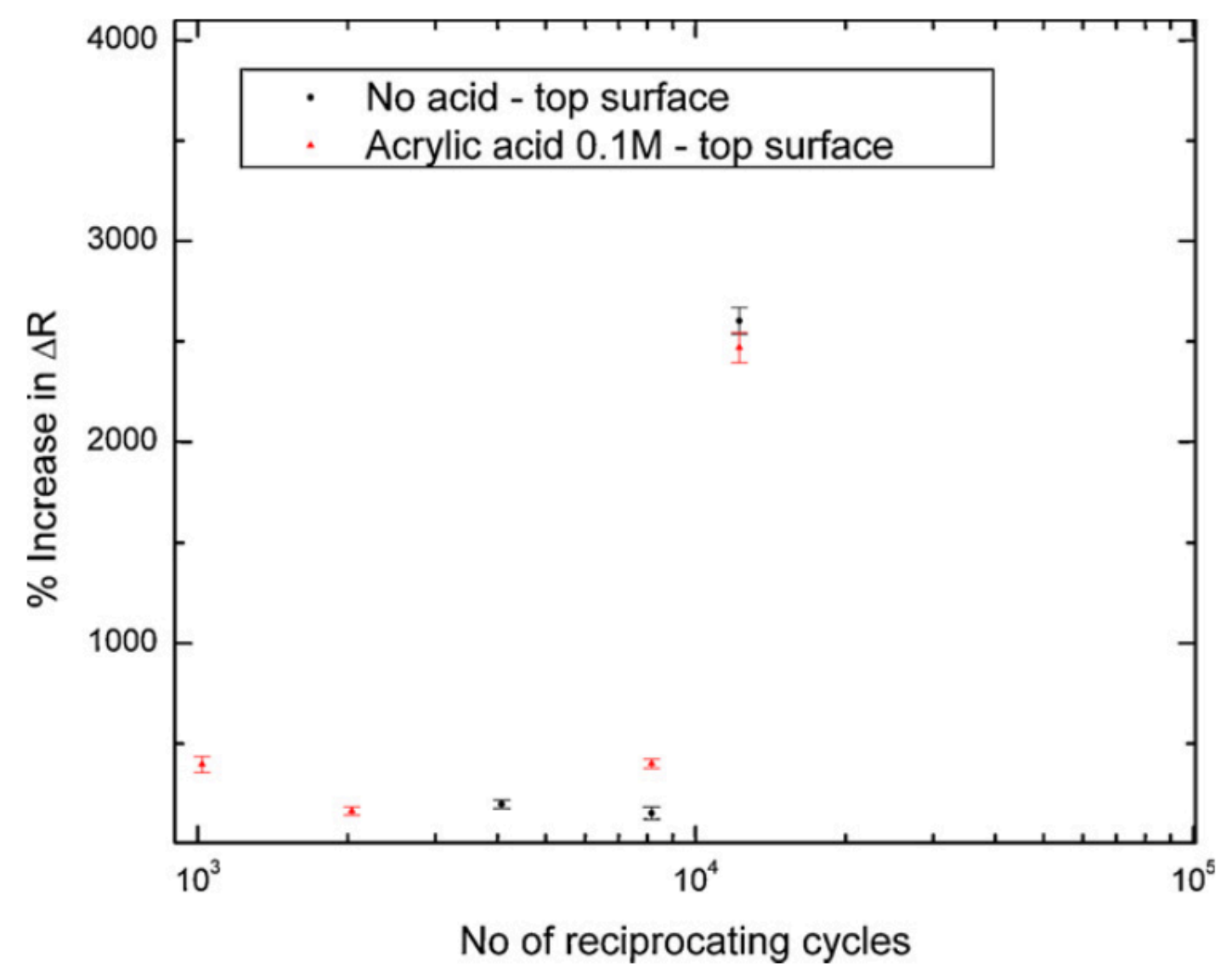

Fig. 5.10 - Percentage increase in electrical resistance, after dry and we sliding, of the top-reciprocating ITO surface against number of sliding cycles (semi-log plot).

Below 10,000 reciprocating cycles the percentage increase in resistance is observed to be between 100 and 500\%. This indicates a significant increase in resistance and consequently a significant functional failure of the top ITO coating. This graph describes $\%$ increase of resistance after testing. The most important, and where we are focusing, is that above 10,000 cycles a large resistance increase, $2500 \%$, is observed for both wet and dry conditions. It is therefore important to expect an increased wear of the ITO coating 
above the 10,000 reciprocating cycles threshold. The acrylic acid solution seems to play an important role protecting, initially, the bottom surface, which is stationary. It is not observed to play such an important role, other than corrosion, when one considers the top, reciprocating surface.

\subsubsection{Weight Measurements}

Weight measurements can be an important tool in determining the wear of coated thin systems. During this study weight measurements were performed before and after each experiment. Fig. 5.11 shows the weight loss variations for both wet and dry sliding of the top-reciprocating ITO surface.

It is important to note that a correlation between the percentage increase in resistance and the weight loss measurements is observed for the top-reciprocating ITO surface, both in the presence of and without the corrosive medium. Above 10,000 reciprocating cycles (in particular at 12,240 cycles or $6 \mathrm{~h}$ of continuous sliding) a significant increase in wear of ITO is observed. This is in agreement with the resistance trend shown in Fig. 5.10. 


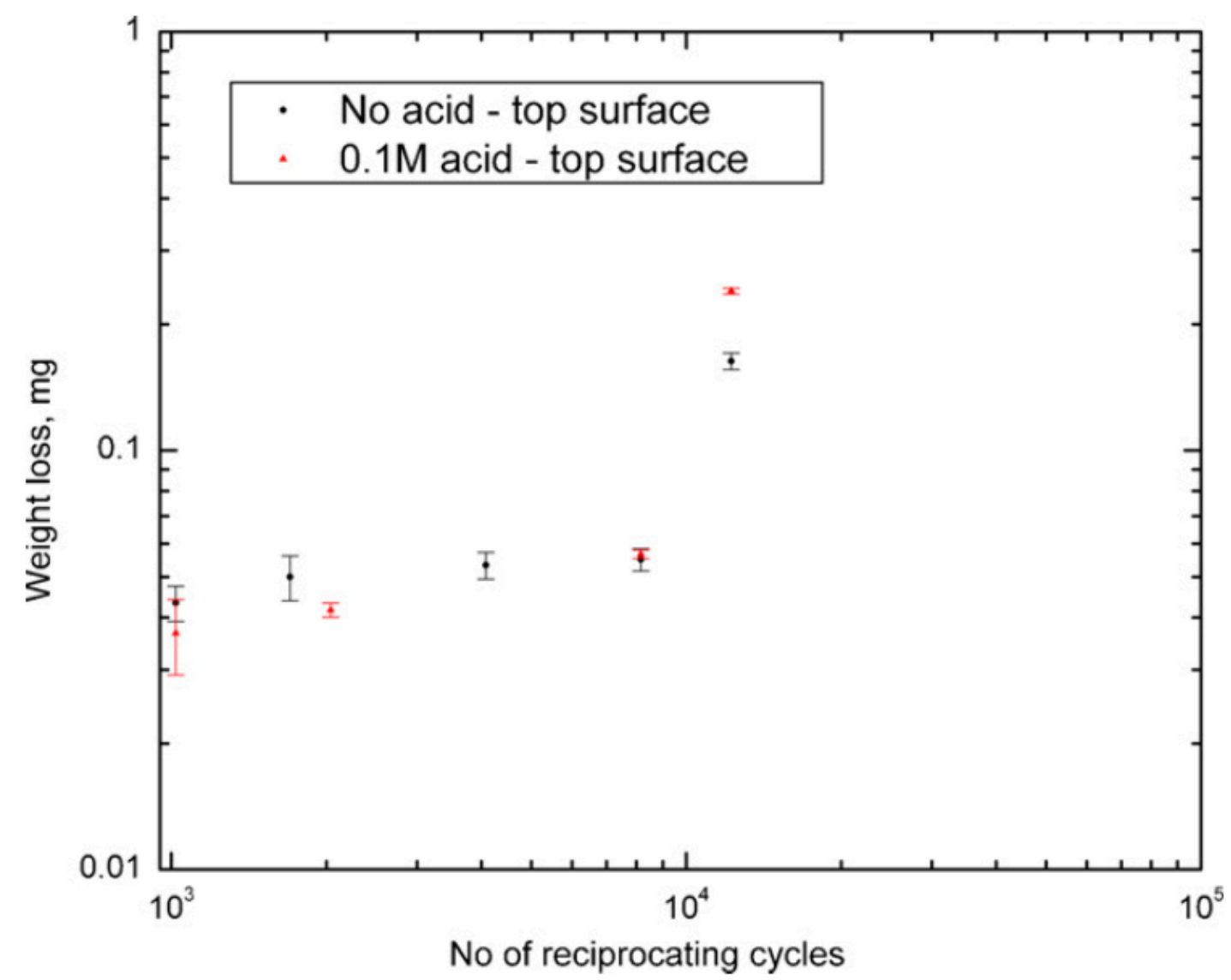

Fig. 5.11 - Weight loss versus number of reciprocating cycles (log-log plot) for both dry and wet sliding of the top ITO surface.

Furthermore, weight measurements were performed also in the case of the bottom ITO counterface in both wet and dry conditions, as presented in Fig. 5.12. 


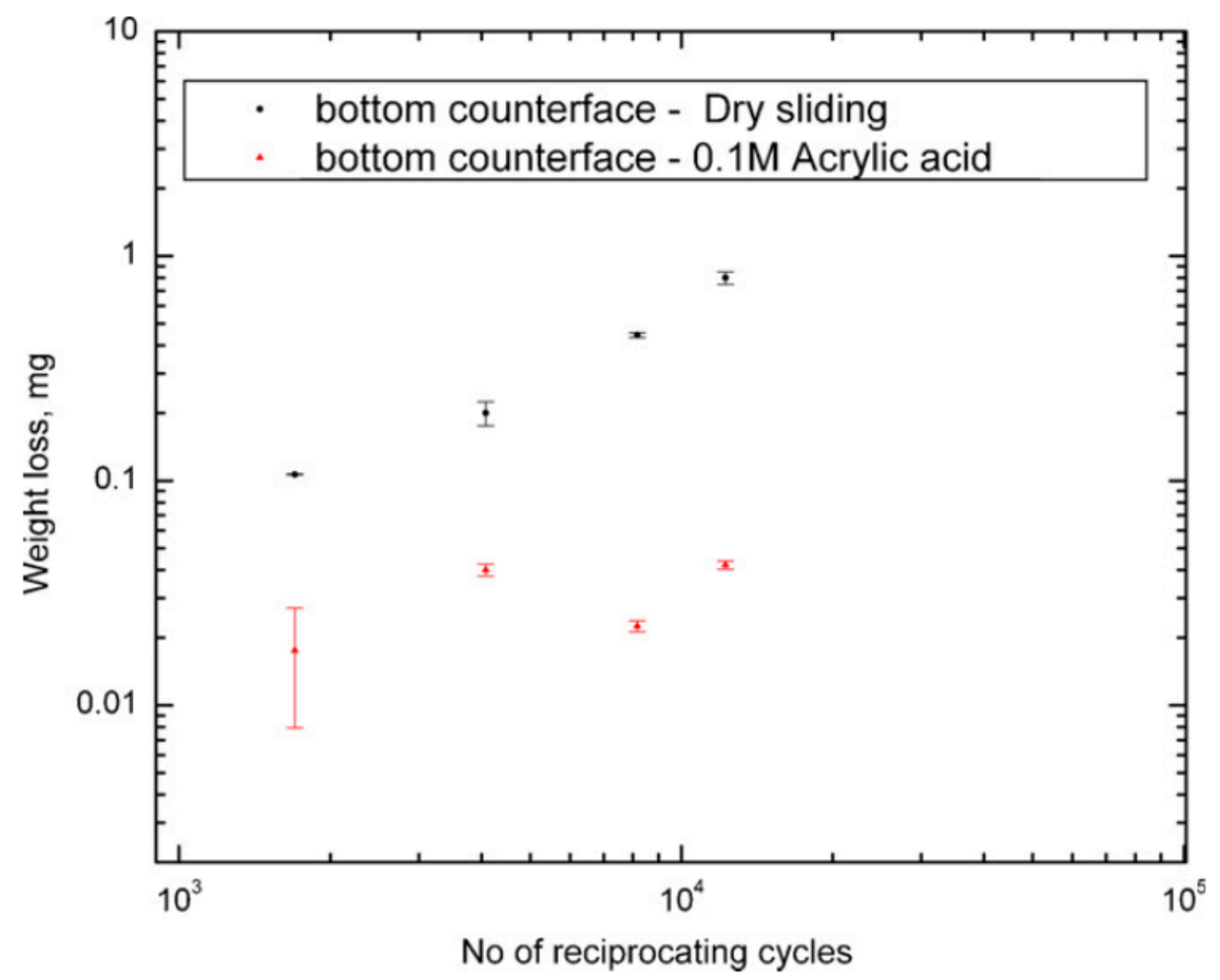

Fig. 5.12 - Weight loss versus number of reciprocating cycles (log-log plot) for both dry and wet sliding of the bottom ITO surface.

More wear, in the form of weight loss, is observed for the case of dry sliding whereas in the case of wet sliding the acrylic acid solution seems to inhibit large wear losses. For the dry sliding condition, the weight loss is increased with increasing number of reciprocating cycles. It is also evident that the corrosive effect of the acidic solution is not so pronounced. The combined action of sliding and corrosion is not observed to be as damaging as the dry sliding itself. The action of the acrylic acid as a protective film cannot be ignored. Also, the difference in behavior of the top and bottom ITO surfaces, indicates that the acrylic acid mostly protects the bottom stationary surface. 


\subsubsection{Surface Profilometry}

Contact mode surface profilometry can provide a powerful tool in determining wear rates of materials in certain cases. However, in this study this is not the case since the top ITO surface moves forwards and backwards along the bottom sample surface leaving a welldefined track of controlled length. The reciprocating motion is produced by a cam drive and therefore the sliding speed varies continuously along the track length from zero at each end to a maximum in the middle. As mentioned by Bull [132], this can lead to cases where a wear transition is observed in only part of the track. This was also confirmed in our case using an optical microscope. This leads to mixed-mode failures of the surface and to a difficulty in interpreting the wear data by surface profilometry, since it is not reasonable to assume a uniform wear track. The situation is further complicated by the presence of the underlying polyester soft substrate. Surface profiles of the bottom ITO surface in both wet and dry sliding conditions reveal significant polymer deformation and protrusions above the surface. This is shown in Fig. 5.13.
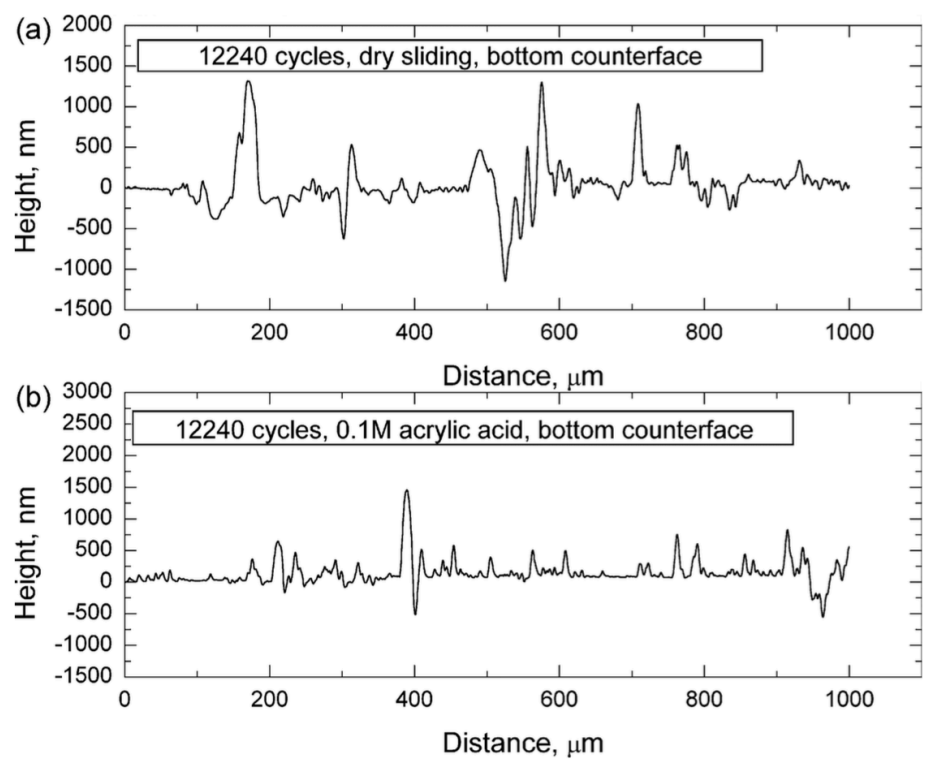

Fig. 5.13 - Typical surface profiles of bottom worn ITO counterface for both dry (a) and wet (b) conditions, after 12,240 reciprocating cycles. 
Therefore it is important to note that in this study weight measurements are the key tool in determining the wear of the various ITO-coated systems.

\subsubsection{Microscopy}

Cohesive and adhesive failure of the ITO coating is noted when the left hand side image of Fig. 5.14 is observed. Also, total removal of ITO coating is observed in large areas of the image.

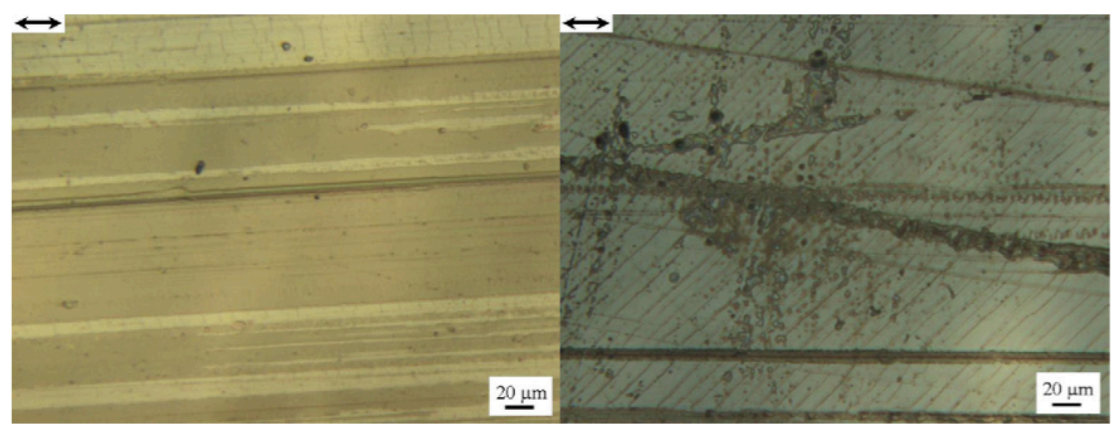

Fig 5.14 - (Left) optical image showing a top ITO counterface for 12,240 cycles under pure dry sliding conditions. (Right) optical image showing a top ITO surface sliding for 12,240 cycles in the presence of 0.1 $\mathrm{M}$ acrylic acid. (Arrows indicate the reciprocating direction).

The right hand side image in Fig. 5.14 shows relatively light corrosion on various areas of the ITO surface. Also, cohesive brittle coating failure is observed in the form of cracks oriented at $45^{\circ}$ with respect to the reciprocating direction. The $45^{\circ}$ angle may indicate the significance of the shear stress developed in the interface of the two sliding ITO surfaces. Total ITO adhesive failure is observed in some areas. 


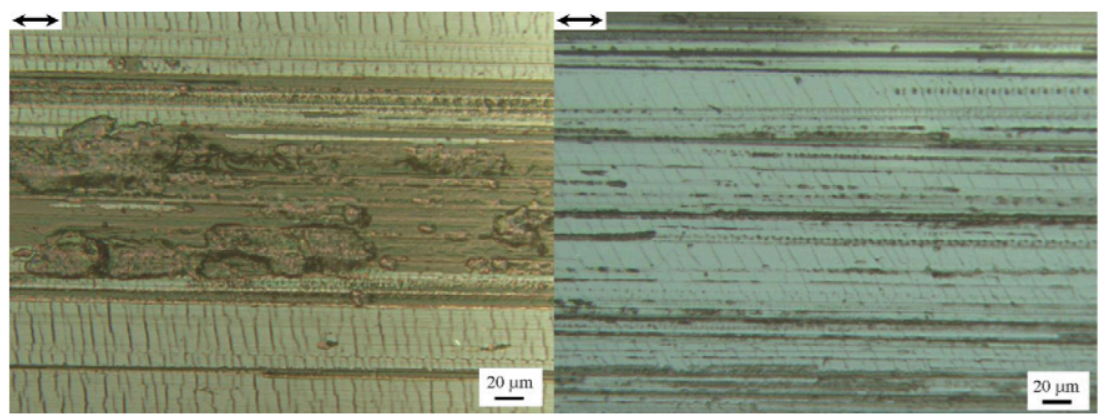

Fig. 5.15 - (Left) Optical image showing a bottom ITO counterface for 12,240 cycles under pure dry sliding conditions. (Right) optical image showing a bottom ITO counterface for 12,240 cycles in the presence of $0.1 \mathrm{M}$ acrylic acid. (Arros indicate the reciprocating direction).

The left hand side image in Fig. 5.15 shows cracking of the ITO coating perpendicular to the sliding direction. Also, adhesive ITO failure is shown clearly. In addition, plastic deformation of the underlying polymer substrate is observed. During wear of ceramic materials, brittle fracture is expected in some cases [23].

Similar wear mechanisms are observed in the right hand side optical image; cohesive and adhesive failure of the ITO coating seem to be the leading failure mechanisms. The coating brittle cracks form a $45^{\circ}$ angle with the reciprocating sliding direction.

Scanning electron microscopy can provide a useful and more in detail insight into the wear mechanisms of the ITO surfaces. The left hand side image in Fig. 5.16 shows the brittle nature of ITO fracture in detail. 


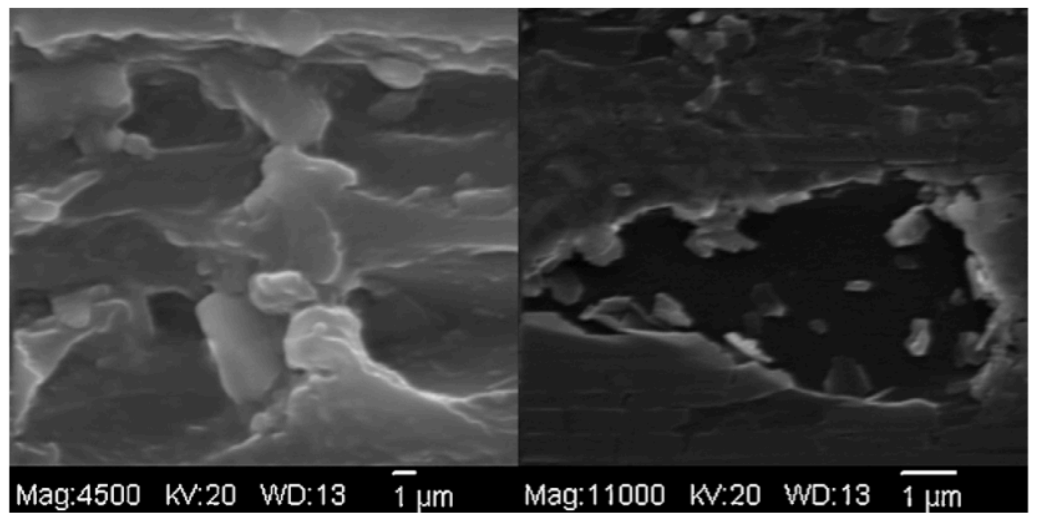

Fig. 5.16 - (Left) SEM image showing a bottom ITO counterface for 1020 cycles in the presence of $0.1 \mathrm{M}$ acrylic acid. (Right) SEM image showing a bottom ITO surface for 1020 cycles under dry conditions.

Some entrapped ITO particles are also visible. The right hand side image in Fig. 5.16 depicts the adhesive failure of an ITO fragment. Some ITO debris is still present within the delaminated area. Coating cracks are also visible in the periphery of the delaminated area.

\subsection{Conclusions}

This chapter focuses on the long-term durability of ITO-coated PET electrodes subjected to combined tribo-chemcial stresses. TOF-SIMS data confirm that the ITO film interacts with an acrylate-acid containing PSA. Also, it is shown that the combination of stress and corrosion by acrylic acid can cause ITO cracking to occur at stresses less than a quarter of those needed for failure with no corrosion.

In addition, the time to failure, under $\sim 1 \%$ tensile strain can reduce the total time to failure by as much as a third. It is also worth noting that corrosion is formed around rough ITO areas. However, the failure of the ITO functional layer is corrosion dominated and the externally-applied mechanical strain is observed to be a contributory failure mechanism. In addition, in $0.1 \mathrm{M}$ acrylic acid, considerable etching of relatively large 
ITO surface areas is observed.

Tribo-chemical results show changes in electrical resistance are correlated with changes in the ITO surface due to friction and wear in both wet and dry sliding conditions. Above the 10,000 reciprocating cycles threshold, an abrupt increase in resistance of the bottom counter-face was observed for both wet and dry sliding conditions. It is believed the acrylic acid solution initially forms a protective low friction wet layer on the bottom ITO counter-face and this leads to limited wear as opposed to the respective dry sliding condition. A correlation between the percentage increase in resistance and the weight loss measurements was noted for the top-reciprocating ITO surface. Weight loss under dry sliding conditions in the case of the bottom counterface sample initially measured to be around $0.1 \mathrm{mg}$ and increase up to $1 \mathrm{mg}$ with increasing number of reciprocating cycles. On the other hand, weight loss for the same sample under wet conditions were less with a maximum value of around of $0.04 \mathrm{mg}$ after 12,240 reciprocating wear cycles being measured. Wear mechanisms of ITO surfaces include cohesive failure within the film, adhesive wear between ITO film and PET substrate, underlying plastic deformation of the PET substrate and abrasive wear of the ITO film. 


\section{Tribo-mechanical studies of zinc oxide films for use in flexible piezoelectric devices}

\subsection{Overview}

This chapter describes tribo-mechanical durability investigations of piezoelectric $\mathrm{ZnO}$

films, typically used in energy harvesting devices. Mechanical controlled buckling experiments were conducted on $\mathrm{ZnO}$-coated PET and PEN films, similar to those conducted on ITO-coated polyester in Chapter 4. Additionally, reciprocating wear testing on $\mathrm{ZnO}$ films was performed using both point and line contact stainless steel rolling/sliding counterfaces. These experiments, in conjunction with in situ and ex situ film characterization techniques were used to predict the sustained functionality of these films for use in flexible energy harvesting devices. The work presented in this chapter has been published in Thin Solid Films, doi: 10.1016/j.tsf/2010/07.081 and doi: 10.1016/j.tsf/2013.05.149.

\subsection{Introduction}

When utilizing $\mathrm{ZnO}$ coatings in tactile harvesting device architectures, it is important to study their tribological properties since it is expected that they will be subjected to rolling/sliding contact-based stresses and reciprocating motions. There are no studies to date which address the mixed rolling-sliding dry tribological behavior of $\mathrm{ZnO}$ film surfaces. However, there are relatively few investigations that report on the dry sliding tribological behavior of $\mathrm{ZnO}$ coatings. Prasad et al. [14,57] performed ball-on-flat friction and wear tests on $500 \mathrm{~nm}$ thick PLD-deposited $\mathrm{ZnO}$ coatings. It was found that the films' wear life was approximately 1 million cycles with maintaining a relatively low coefficient of friction range between 0.16 and 0.18 . Lin et al. [63] investigated the 
tribological behavior of sol-gel deposited $\mathrm{ZnO}$ and the effect of annealing temperature. Pin-on-plate wear testing was performed up to 10,000 cycles on non-annealed films and specimens annealed at $450{ }^{\circ} \mathrm{C}, 500{ }^{\circ} \mathrm{C}, 550{ }^{\circ} \mathrm{C}, 700{ }^{\circ} \mathrm{C}$, and $800{ }^{\circ} \mathrm{C}$, respectively. It was observed that with increasing annealing temperature the resulting wear rate was decreasing. Chai et al. [58] measured the coefficient of friction of $\mathrm{ZnO}$ films fabricated by atomic layer deposition. Films were deposited at $150^{\circ} \mathrm{C}$ and $300{ }^{\circ} \mathrm{C}$ and were tribologically tested up to 18,000 cycles. They were found to exhibit relatively low coefficients of friction between 0.11 and 0.12 , and wear rates equal to $8.0 \times 10^{-4}$ $\mathrm{nm} /$ cycle and $1.4 \times 10^{-3} \mathrm{~nm} /$ cycle respectively. Such lubricious performance was attributed to the films' grain size and uniformity. Furthermore, piezoelectric $\mathrm{ZnO}$ coatings are observed to exhibit adequate compatibility to selected micromachining processes [104] and therefore are considered as strong candidates for micro-machined sensors and energy harvesters.

Ambient energy harvesting devices are being recognized as the key in powering adaptive systems and autonomous networks. Piezoelectric $\mathrm{ZnO}$ films can be an inexpensive and versatile active material candidate for the fabrication of such devices. In fact, $\mathrm{ZnO}$ films are already used in cantilever-based mechanical vibration harvesters. However, the development of a design that is non-cantilever-based is required in order to reduce the device shape and size. For this reason, it is of paramount importance to investigate the rolling/sliding characteristics of $\mathrm{ZnO}$ films along with their piezoelectric output.

\subsection{Experimental Methods}

\subsubsection{Materials}

For initial film surface and film characterization, $\mathrm{ZnO}$ thin films were deposited using 
DC magnetron sputtering on $125 \mu \mathrm{m}$ thick biaxially oriented PET (Melinex ST504) and PEN (Teonex Q65FA) substrates. Both substrates have a few nanometers thick acrylateresin layer to promote adhesion. The glass transition temperature as measured by differential scanning calorimetry (Perkin Elmer DSC 7) was equal to $78.8^{\circ} \mathrm{C}$ for PET and $120.2{ }^{\circ} \mathrm{C}$ for PEN. The melting temperature of PET was measured to be $254.6^{\circ} \mathrm{C}$ and that of the PEN substrates as $264.8^{\circ} \mathrm{C}$. The upper processing temperature for PET is typically $150{ }^{\circ} \mathrm{C}$ and for PEN is between $180^{\circ} \mathrm{C}$ and $220^{\circ} \mathrm{C}$ [68]. The degree of crystallinity for the PEN substrates was $36.7 \%$ and for the PET it was $26.46 \%$. The elastic modulus of the substrates in uniaxial tension (Instron 4410) for dog-bone samples (25 $\mathrm{mm} \times 5 \mathrm{~mm}$ ) were $2.35 \pm 0.29 \mathrm{GPa}$ for PET and $3.3 \pm 0.37 \mathrm{GPa}$ for PEN.

Films were deposited by reactive DC magnetron sputtering (Alcatel) in oxygen plasma at a pressure of $8 \times 10^{-3}$ mbar using a $99.99 \%$ pure $\mathrm{Zn}$ metallic target. The chamber base pressure was $5 \times 10^{-7} \mathrm{mbar}$. The film thickness was monitored in situ and ex situ using an Alphastep profilometer. The films were deposited on an acrylate adhesion promoting layer surface of PET and PEN substrates with their thicknesses ranging between $25 \mathrm{~nm}$ and $1 \mu \mathrm{m}$. In each sputtering deposition cycle, one PET and one PEN substrate were used. The substrate temperature during depositions was kept below the respective upper processing temperature for PET and PEN, $120^{\circ} \mathrm{C}$ and $150^{\circ} \mathrm{C}$, respectively.

For reciprocating wear experiments, $\mathrm{ZnO}$ films were deposited on glass substrates using both sol-gel and spray pyrolysis methods. For sol-gel deposition, zinc acetate dihydrate $\left(\mathrm{Zn}\left(\mathrm{CH}_{3} \mathrm{OO}\right)_{2} \cdot 2 \mathrm{H}_{2} \mathrm{O}\right)$ was dissolved in a solution of isopropanol and monoethanolamine (MEA) at room temperature. The molar ratio of MEA to zinc acetate was held at a 1:1 
ratio and the concentration of zinc acetate was equal to $1.0 \mathrm{~mol} \mathrm{~L}^{-1}$. The mixture was then stirred at $60{ }^{\circ} \mathrm{C}$ for 2 hours to yield a clear homogenous solution. The solution was then syringe-filtered using a Fisherbrand $0.45 \mu \mathrm{m}$ polytetrafluoroethylene filter. Gel films were deposited on the substrate by dip coating of soda-lime glass microscope slides of area equal to $75 \times 25 \mathrm{~mm}$. The withdrawal rate was $50 \mathrm{~mm} / \mathrm{min}$. In order to get the desired film thicknesses of $1 \mu \mathrm{m}$ a number of dips were performed. After each dip the films were pre-heated at a temperature of $250{ }^{\circ} \mathrm{C}$ for $10 \mathrm{~min}$ and they were post-heated at $500{ }^{\circ} \mathrm{C}$ for 1 hours. The heat treatments were conducted by placing the samples in an electric furnace at the respective temperatures.

For point-contact rolling/sliding wear testing of pattern $\mathrm{ZnO}$ films, $\mathrm{ZnO}$ was deposited on corning glass substrates via spray pyrolysis at a precursor temperature of $350^{\circ} \mathrm{C}$ with a deposition time of 30 minutes. Aqueous solutions of $\mathrm{Zn}\left(\mathrm{NO}_{3}\right)_{2}$ were diluted to $0.05 \mathrm{M}$ and $0.2 \mathrm{M}$ prior to film deposition. Deposition of the films was conducted using a custom-built spray pyrolysis system develop by Kortidis et al. [148]. The counter-wear surface was 302 stainless steel ball bearings with a diameter of 0.375 inches $(9.525 \mathrm{~mm})$.

$\mathrm{ZnO}$ films were patterned using photolithography and wet chemical etching. An adhesion promoter (Hexamethyldisilazane) and a positive photoresist (AZ 3300-F, AZ Electronic Materials) are deposited on the $\mathrm{ZnO}$ using spin coating deposition. A chrome/quartz photomask featuring various patterns of lines and square is used with an ultraviolet flood exposure lamp $(\lambda=365 \mathrm{~nm})$ to create the desired pattern in the photoresist layer. After developing the photoresist in AZ 3330 developer, the $\mathrm{ZnO}$ samples are etched in a 1:200 
concentration of $\mathrm{H}_{2} \mathrm{O}: \mathrm{HCl}$ for one minute and feature an etch rate of 0.9 microns per minute.

\subsubsection{Contact Angle Goniometry}

Static contact angle measurements were performed to characterize the wetting properties of deposited $\mathrm{ZnO}$ films. Five drops $(\sim 2 \mu \mathrm{L})$ of deionized water were placed on the sample's surface. An image of the five droplets was taken using a fixed microscope. The image was digitally analyzed using the Image J "drop analysis" plugin method LBADSA. Contact angles were measured before UV exposure of the samples and after 15 min, $30 \mathrm{~min}, 45 \mathrm{~min}$, and $60 \mathrm{~min}$ of UV exposure respectively. The specimens were exposed to UV using a NuArc (26-1KS) Exposure System.

\subsubsection{Atomic Force Microscopy}

Atomic force microscopy (AFM) was used to characterize the surface morphology of the sputtered films. These measurements were made with a Nanoscope III atomic force microscope (Digital Co. Instruments, USA) using a normal silicon nitride tip (125 $\mu \mathrm{m})$ in Tapping Mode scanning the surface with an oscillating tip to its resonant frequency (200$400 \mathrm{kHz}$ ). All measurements were made at room temperature. Nanotribological measurements were also conducted before and after $\mathrm{ZnO} \mathrm{UV}$ exposure using a $\mathrm{ZnO}$ coated Si tip.

\subsubsection{Nanoindentation Testing}

Nanoindentation was performed with a Nano-Indentation Tester $\left(\mathrm{NHT}^{2}\right)$ by CSM 
Instruments and the experiments were conducted using a Berkovich tip. A maximum load of $25 \mathrm{mN}$ with a loading/unloading rate of $50 \mathrm{mN} / \mathrm{min}$ and a 10 second pause between loading and unloading segments was used. Experiments were performed to determine effects of patterning and initial solution concentration on modulus of elasticity and indentation hardness of the $\mathrm{ZnO}$ films.

\subsubsection{Scratch Testing}

Scratch testing was performed using a $100 \mu \mathrm{m}$ radius spherical diamond indenter. Samples were adhered to glass slides for testing and the applied load was increasing linearly. Relatively low-load scratching was performed using the high-resolution cantilever of the nanoscratch tester (CSM Instruments). Critical loads for coating failure were determined using in situ optical microscopy.

\subsubsection{Controlled Buckling}

The controlled compression or buckling test has been described in Chapter 4. In this work the externally applied buckling strains were calculated using the formula described in the work of Chen et al. [90]. When coupled with in situ optical microscopy it is a straightforward way to measure the film's critical onset strain for cracking and the progression of the failure as strain increases. In situ optical monitoring can be highly beneficial in cases when the film is not conductive.

\subsubsection{Reciprocating Wear}

Macro-tribological experiments were performed using a custom-built reciprocating wear 
tester. The counter-surface used was a stainless steel cylinder, with dimensions of 15.9 $\mathrm{mm}$ in diameter and $25 \mathrm{~mm}$ in length. The cylinder experienced a mixed rolling-sliding motion as it reciprocated along the surface of test samples. The reciprocating length was equal to $35 \mathrm{~mm}$. Stainless steel was selected because of its moderate coefficient of friction and its structural rigidity. A relatively moderate, contant load of $2.5 \mathrm{~N}$ was applied normally to the reciprocating direction. The rolling frequency was set equal to 0.5 $\mathrm{Hz}$ for all tests. Experiments were conducted at various numbers of reciprocating cycles until the coating was observed to be completely removed. The line-contact rolling-sliding apparatus can be seen in Fig. 2.5. In addition, tests were performed on samples both exposed and non-exposed to UV light. The electrical output of the piezoelectric devices was monitored in situ.

Combined rolling-sliding using a point contact counterface comprised of a 302 stainless steel ball and the $\mathrm{ZnO}$ bottom counterface was achieved using the custom-built reciprocating wear tester. A custom ball holder was fabricated with a stainless steel cup to retain the ball, while allowing rolling and sliding translation of the ball during reciprocation. A constant normal load of $2.3 \mathrm{~N}$ was applied. Each experiment was conducted using a fixed reciprocating speed of 0.1 cycles/sec. Each reciprocating wear cycle was equal to $18 \mathrm{~mm}$ of linear sliding distance. Wear tests were run up to five hundred cycles. Fig. 2.6 shows a representation of the custom build reciprocating wear apparatus.

\subsubsection{Weight Measurements}

Weight measurements were conducted before and after each test, to monitor potential film wear losses, using a digital balance (DV215CD, Ohaus, $0.01 \pm 0.02 \mathrm{mg}$ ). After 
testing and before weighing the samples were washed in acetone, IPA, and finally distilled water, and they were then dried using a hot plate set to $120^{\circ} \mathrm{C}$.

\subsubsection{Microscopy}

Optical images of both worn sample and counter surfaces were taken using a Leica optical microscope equipped with a frame grabber (Guppy, Allied Visions Technology) at magnifications of $100 \times$ and $200 \times$. A scanning electron microscope (SEM Hitachi S4000 ) with a field emission gun was employed in order to perform ex situ observations of the tested film surfaces. The imaging was conducted using an operating voltage of $20 \mathrm{kV}$. Samples were sputtered with a $5 \mathrm{~nm}$ gold coating (Hummer X) prior to SEM observation. Contact mode surface profilometry on a Veeco Dektak 110 surface stylus profilometer, was used to scan the wear track scars with a $12.5 \mu \mathrm{m}$ stainless steel stylus tip. Under a constant scanning load of $10 \mathrm{mg}$, scan lengths of $1 \mathrm{~mm}$, and a scanning time of 20 seconds, profiles of wear tracks at various numbers of cycles were produced.

\subsection{Results and Discussion 6.4.1 Surface Characterization}

Contact angle gonionmetry was performed to characterize the wetting properties of PET and PEN surfaces. Fig. 6.1 shows a bar graph that presents the contact angle values for the two different substrate surfaces. 


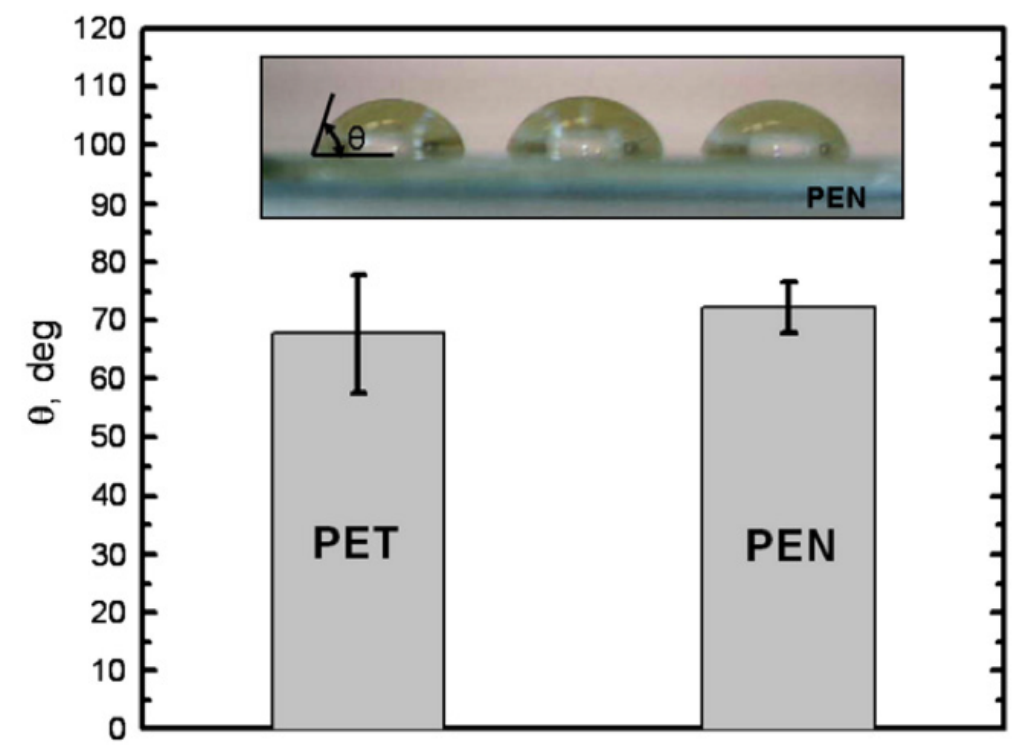

Fig. 6.1 - Contact angle values of PET and PEN substrate surfaces. Error bars indicate standard deviation of data. Inset image shows a typical contact angle measurement on a PEN surface.

The PEN proved to be slightly more hydrophobic and thus deposited $\mathrm{ZnO}$ films were expected to exhibit slightly lower adhesion than when deposited on the PET surface, which exhibits a slightly lower contact angle, but higher variability. Atomic force microscopy was performed on $\mathrm{ZnO}$ films deposited on PET substrates. The $\mathrm{ZnO}$-coated PET films were chosen due to their better adhesion than those deposited on PEN. A range of thickness between $65 \mathrm{~nm}$ up to $680 \mathrm{~nm}$ was used. Previous studies [149] have shown that the growth of $\mathrm{ZnO}$ films is columnar with an increasing grain size as thickness increases. This was confirmed using AFM characterization, presented in Fig. 6.2. 

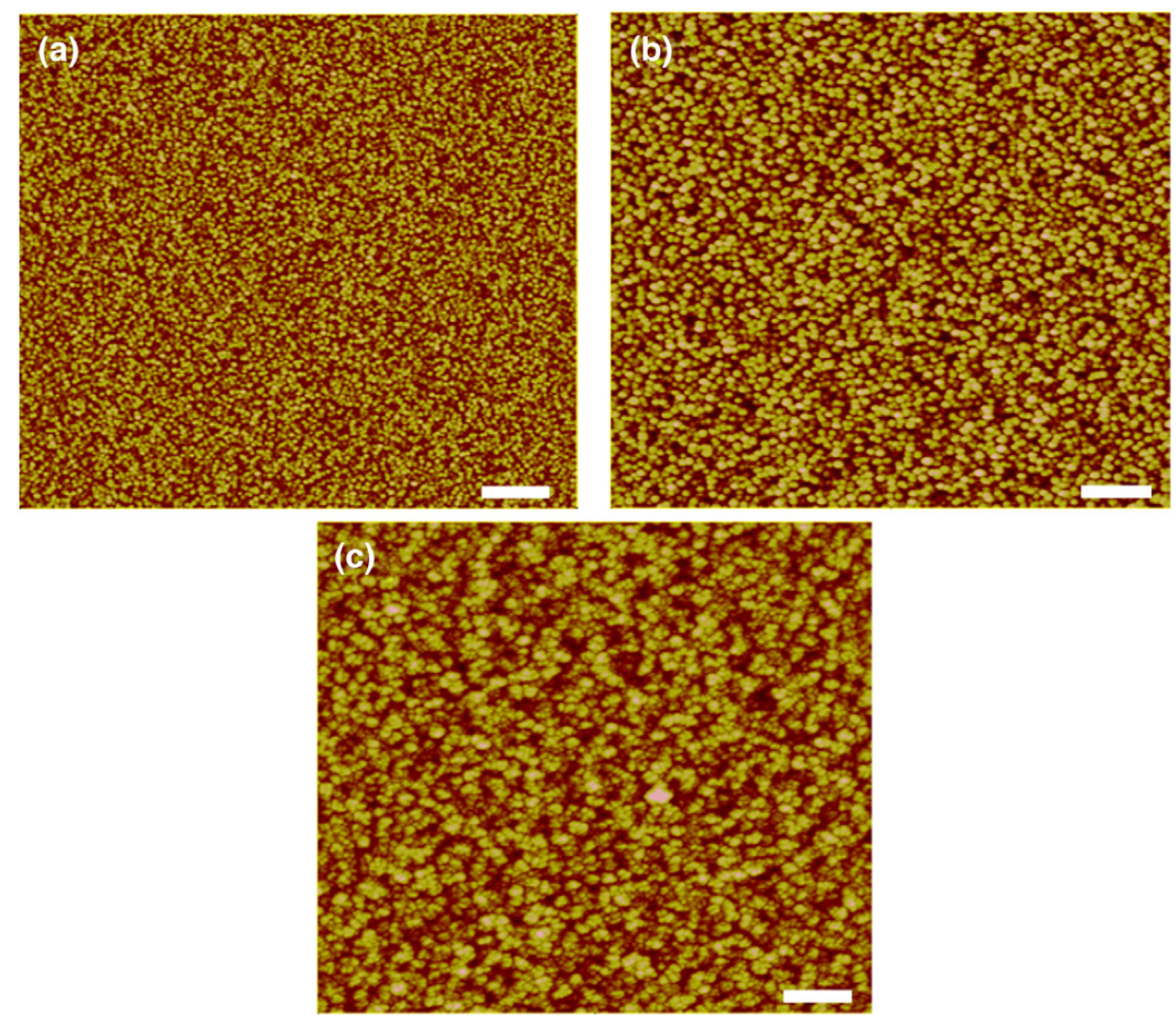

Fig. 6.2 - AFM images of $\mathrm{ZnO}$ films sputtered on PET. a) $\mathrm{ZnO}$ thickness is $65 \mathrm{~nm}$ and Z-range is $28 \mathrm{~nm}$. b) $\mathrm{ZnO}$ thickness is $325 \mathrm{~nm}$ and Z-range is $76 \mathrm{~nm}$. c) $\mathrm{ZnO}$ thickness is $680 \mathrm{~nm}$ and Z-range is $74 \mathrm{~nm}$. The scale bar is equal to $650 \mathrm{~nm}$ in all cases.

At the thickness of $680 \mathrm{~nm}$ additional film material is also observed to form between the columnar grains (Fig. 6.2c).

The effect of UV exposure on the $\mathrm{ZnO}$ surface was an area of importance during this investigation. In Fig. 6.3 a graph of the contact angle measurements at different UV exposure times is presented. 


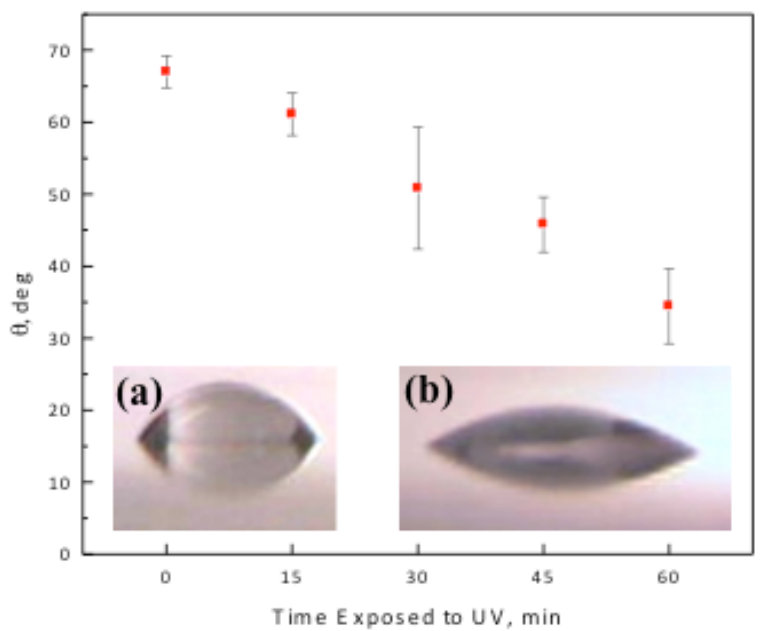

Fig. 6.3 - Contact angle values for $\mathrm{ZnO}$ versus different UV exposure times, a) water droplet on a non-UV exposed $\mathrm{ZnO}$ surface, b) water droplet on a 60 minute $\mathrm{UV}$ exposed $\mathrm{ZnO}$ surface.

It was observed that increased UV exposure time increases the hydrophilic properties of the $\mathrm{ZnO}$ films. This leads to increased adhesion and can be an indirect method of controlling the frictional response of the $\mathrm{ZnO}$ surfaces. An increased coefficient of friction associated with longer exposure times was expected due to the increased adhesion component. Higher interatomic forces may be observed between the tribological pair. These forces may also be expected to lead to increased frictional heating and thus friction-induced energy losses of the tribological system.

AFM measurements, Fig. 6.4, were performed on these $\mathrm{ZnO}$ surfaces. Experiments were designed as a 'single asperity' model, mimicking a triboloical case of a single- point wear/friction counterface. Samples were characterize in their natural state, and after exposure to UV radiation for one hour. 

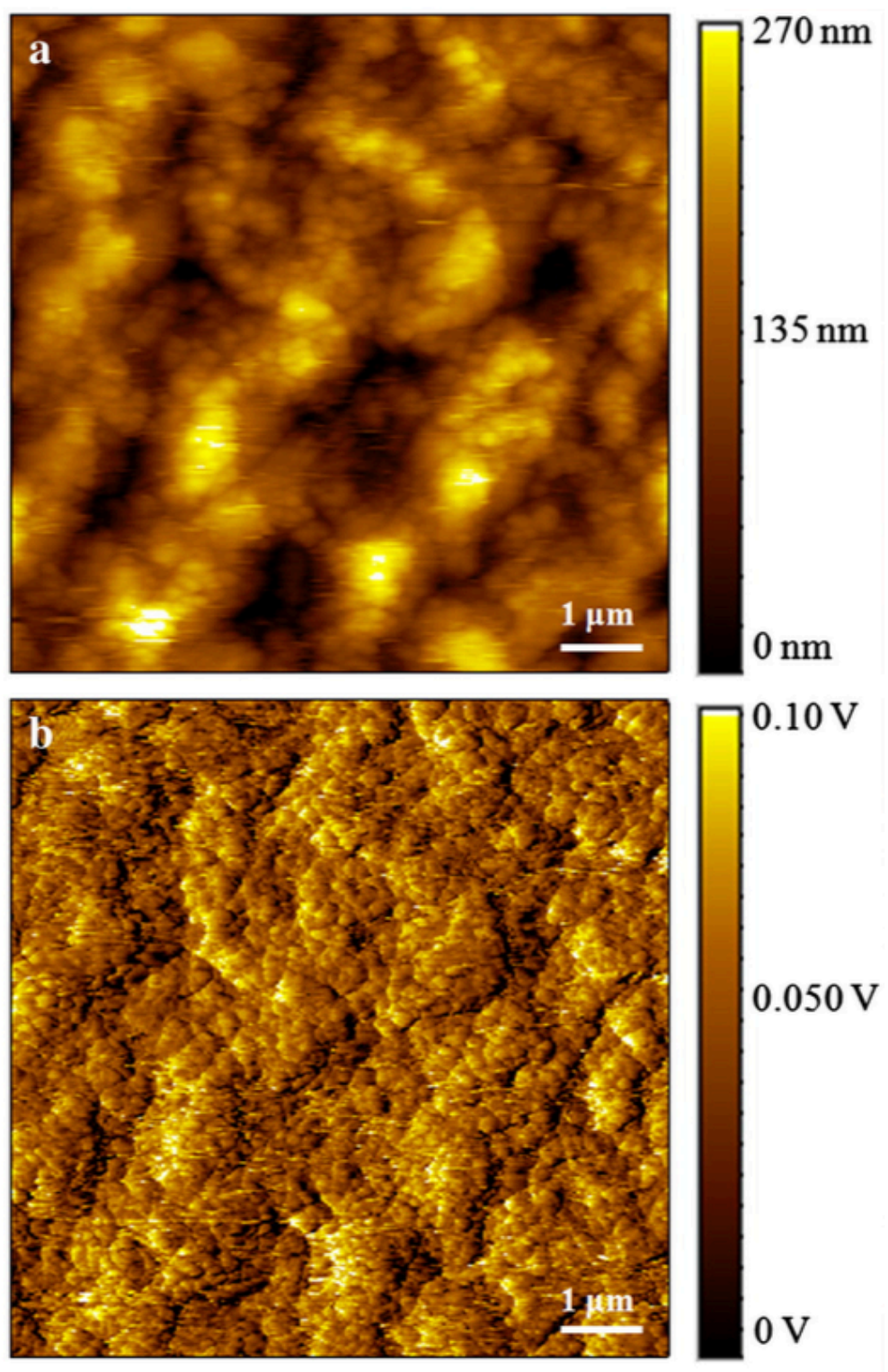

Fig. 6.4 - a) AFM topographical and b) friction map images of UV exposed $\mathrm{ZnO}$ surface for $60 \mathrm{~min}$. Fig. 6.4b presents a frictional map of the UV-exposed surface indicates higher friction values near the high surface asperity peaks. The trough regions were observed to exhibit lower frictional behavior. This can be attributed to the elastoplastic junctions formed between the silicon counterface nano-asperities and the $\mathrm{ZnO}$ surface asperity peaks. Asperity interlocking may also contribute to the increased frictional response of such areas. 
Fig. 6.5c depicts the $\mathrm{ZnO}$ film frictional response before and after UV exposure.

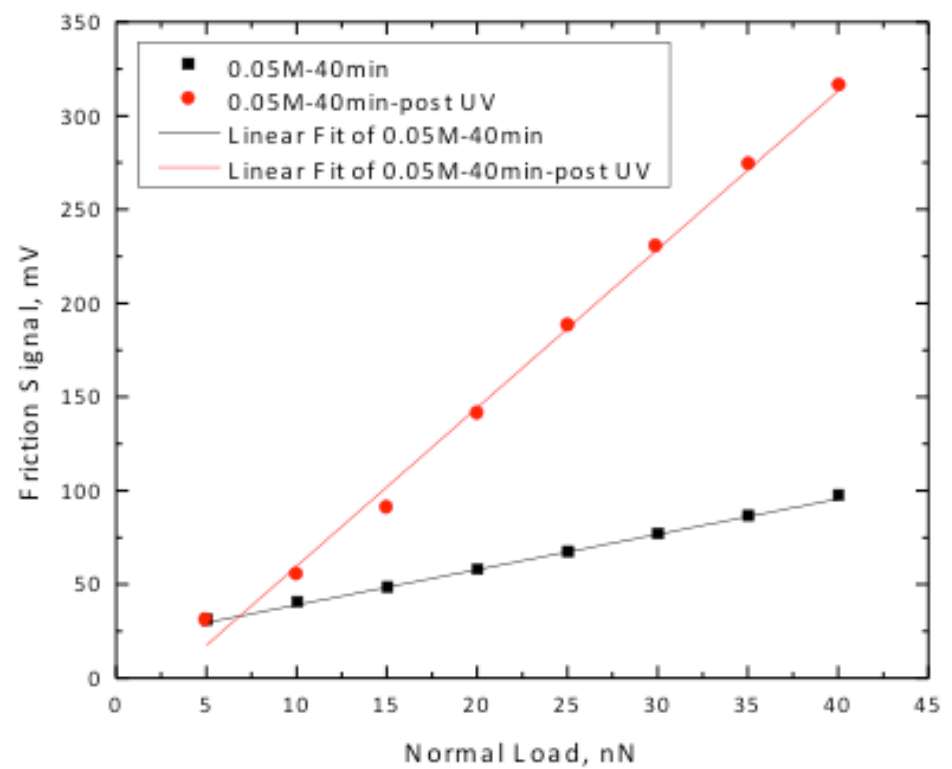

Fig. 6.5 - Friction signal versus applied normal load for $\mathrm{ZnO}$ single asperity sliding against a $\mathrm{ZnO}$ film surface before and after exposure to UV light for 60 minutes.

The measured relative friction coefficient, when $\mathrm{ZnO}$ slides on $\mathrm{ZnO}$, was observed to increase from 0.097 to 0.421 upon exposure of the surface to UV irradiation for $60 \mathrm{~min}$.

\subsubsection{Film Characterization}

The optical transmission characteristics of the $\mathrm{ZnO}$ films deposited on PET substrates were measured and the results are presented in Fig. 6.6. 


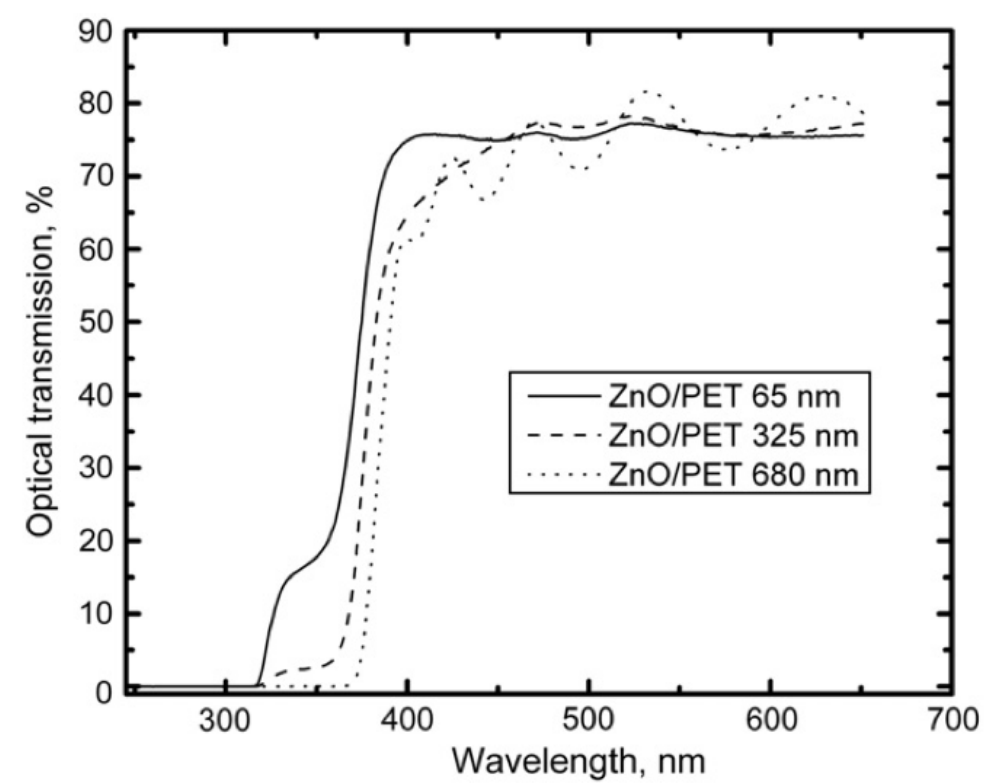

Fig. 6.6 - Optical transmission versus wavelength for various thicknesses of ZnO-coated PET.

It was observed that optical transmission is not thickness dependent for film thicknesses ranging from $65 \mathrm{~nm}$ to $680 \mathrm{~nm}$. In all cases, optical transmission values of around $80 \%$ were measured in the visible spectrum. The observed shift of the absorption edge, at wavelengths between $315 \mathrm{~nm}$ and $370 \mathrm{~nm}$, as a function of thickness was attributed to a decrease in the film band-gap.

Fig. 6.7 shows a typical XRD pattern for a sol-gel deposited $\mathrm{ZnO}$ film at the conditions described previously. 


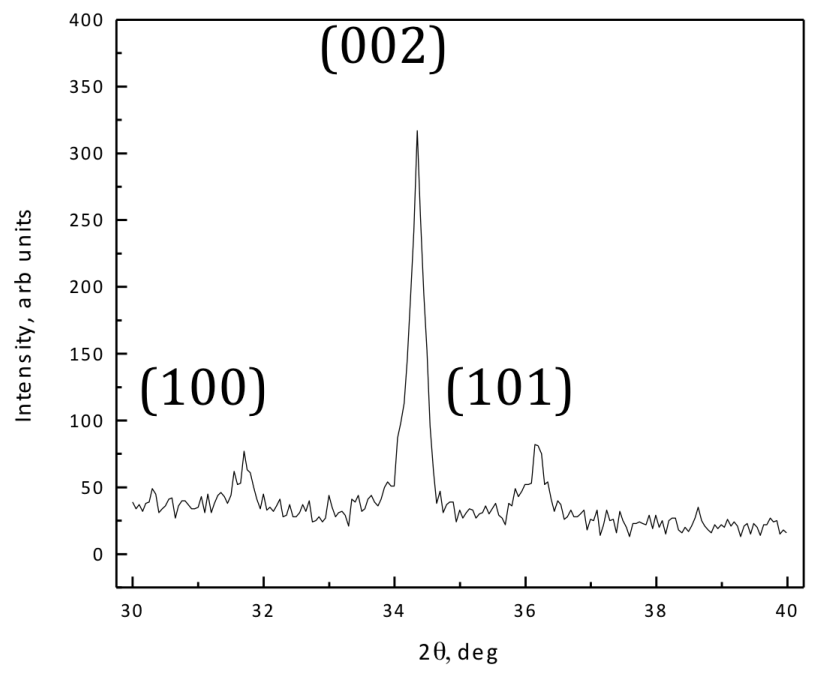

Fig. 6.7 - X-ray diffraction pattern of a $2 \theta$ scan for a sol-gel deposited $\mathrm{ZnO}$ film using a zinc acetate dehydrate solution.

It was observed that the film exhibits a strong c-axis (002) orientation peak, which is known to indicate piezoelectric film properties [60]. It is also observed that there are small diffraction peaks that correspond to (100) and (101).

\subsubsection{Nanoindentation}

Nanoindentation experiments were performed to measure the indentation hardness and elastic modulus of sol-gel derived $\mathrm{ZnO}$ films. A maximum load of $0.5 \mathrm{mN}$ was determined to be sufficient for obtaining reliable data and not exceeding the maximum penetration depth allowed. Matrices of indents were performed on several areas of the film surface. All indents were performed with a standard linear loading/unloading time of 30 seconds and a 10 second pause. The $\mathrm{ZnO}$ films' hardness and elastic modulus were measured to be $3.86 \pm 0.47 \mathrm{GPa}$ and $81.31 \pm 9.97 \mathrm{GPa}$ respectively. Representative indentation curves for the $\mathrm{ZnO}$ films are shown in Fig. 6.8. 


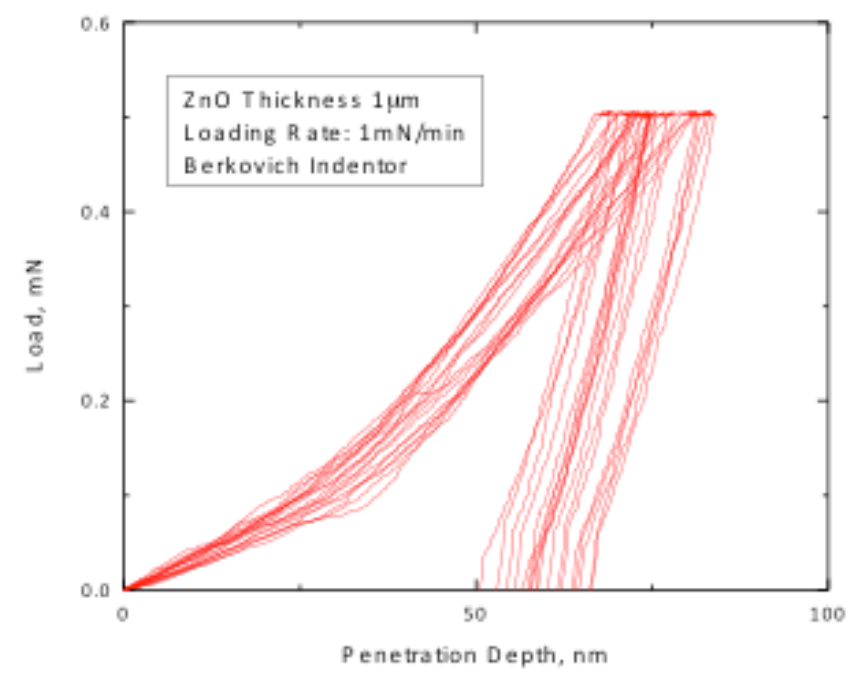

Fig. 6.8 - Load versus penetration depth indentation curves for $1 \mu \mathrm{m}$ thick $\mathrm{ZnO}$ films. A Berkovich diamond tip was used.

The measured values of modulus and hardness were comparable to a previous study of mechanical properties of sol-gel deposited $\mathrm{ZnO}$ films [63].

\subsubsection{Scratch Testing}

Scratch testing of $\mathrm{ZnO}$ films on polyester substrates using a diamond indenter and under a linearly increasing normal load was conducted in order to study the scratch failure mechanisms and detect the critical normal loads at which they occur. For both substrates two major mechanisms were observed using in-situ optical microscopy as shown in Fig. 6.9 . 


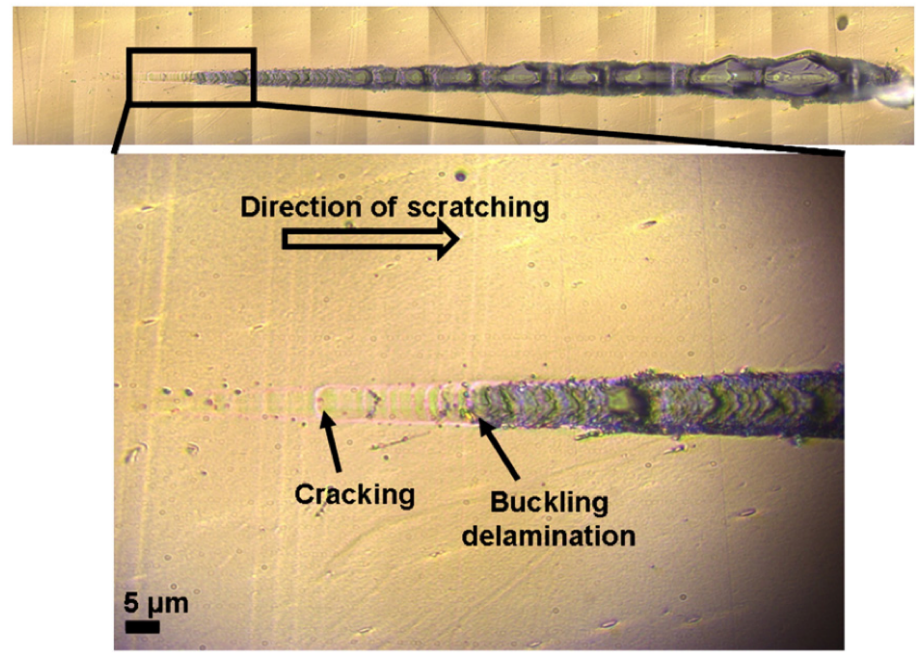

Fig. 6.9 - Optical images showing a panoramic view of the scratch which has a total length equal to 0.5 $\mathrm{mm}$ (top) and the major scratch failure mechanisms (bottom) for a $75 \mathrm{~nm} \mathrm{ZnO}$ coated PET sample.

Initial film failure was observed in the form of through thickness film cracking as a result of the $\mathrm{ZnO}$ film bending into the scratch track due to the deformation of the underlying substrate. Tensile cracks can be developed behind the indenter. This was the dominant mechanism in hard coating/soft substrate systems [132]. The second mechanism, which was observed relates to buckling and delamination of the coating. The coating buckles ahead of the indenter, due to the plowing of the underlying substrate and this subsequently leads to interfacial failure and delamination from the substrate. The buckling delamination mechanism was a second critical normal load. Fig. 6.10 shows the critical load for coating cracking for different thicknesses of $\mathrm{ZnO}$ coated PET and PEN substrates. 


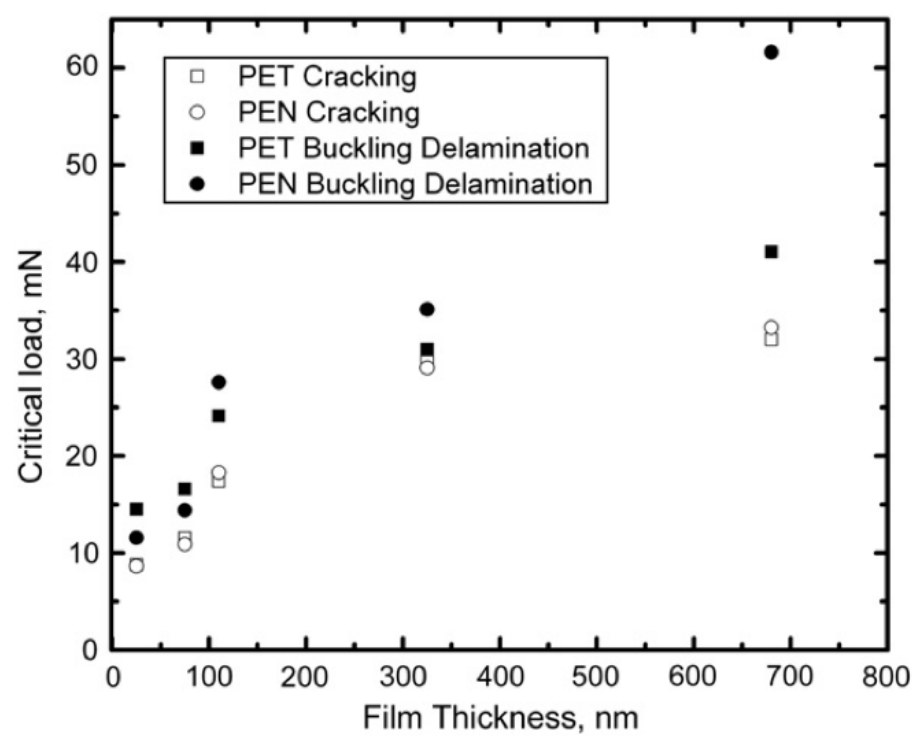

Fig. 6.10 - Critical normal loads for cracking and delamination as a function of film thickness during scratch testing.

Critical load values follow a similar trend for both substrates. This indicates that the cracking mechanism is mostly dependent on the thickness of the coating. The normal load to cause cracking increases with increasing coating thickness. However, for films of approximately $680 \mathrm{~nm}$, the increase in load is not observed to be as pronounced as it is observed in the thickness range from 25 to $325 \mathrm{~nm}$. It can therefore be postulated that in the large thickness regime there is a critical coating thickness above which the effect of thickness on the cracking mechanism is reduced. The second critical normal load at which buckling delamination occurs and its relation to coating thickness is also shown in Fig. 6.10. It is observed that as thickness increases, the load to cause coating buckling delamination also increases for both PET and PEN. Fig. 6.11 shows an SEM image of a part of the scratch track beyond the point of buckling delamination failure. 


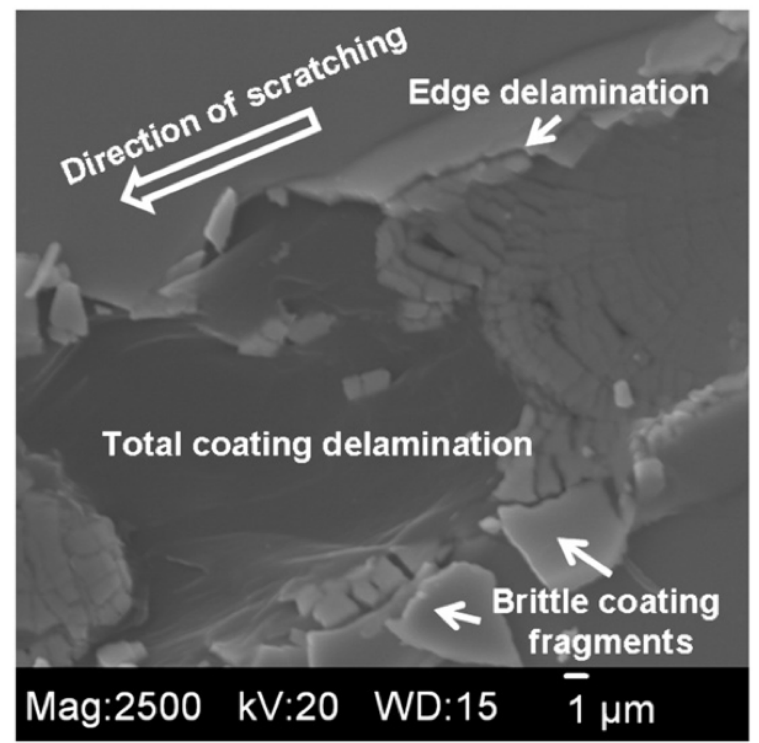

Fig. 6.11 - SEM image showing secondary failure mechanisms for a $650 \mathrm{~nm} \mathrm{ZnO}$ film deposited on a PET substrate.

The coating appears to be crushed into the surface of the track by the moving indenter with an area of total coating delamination to follow. Plastic deformation of the underlying polymer substrate is also evident in this area. Track edge delamination and brittle coating fragments are also observed.

\subsubsection{Controlled Buckling of $\mathrm{ZnO}$ films}

During in situ compression testing, the $\mathrm{ZnO}$ surface is deformed under tension and a typical cohesive failure progression is shown in Fig. 6.12. 


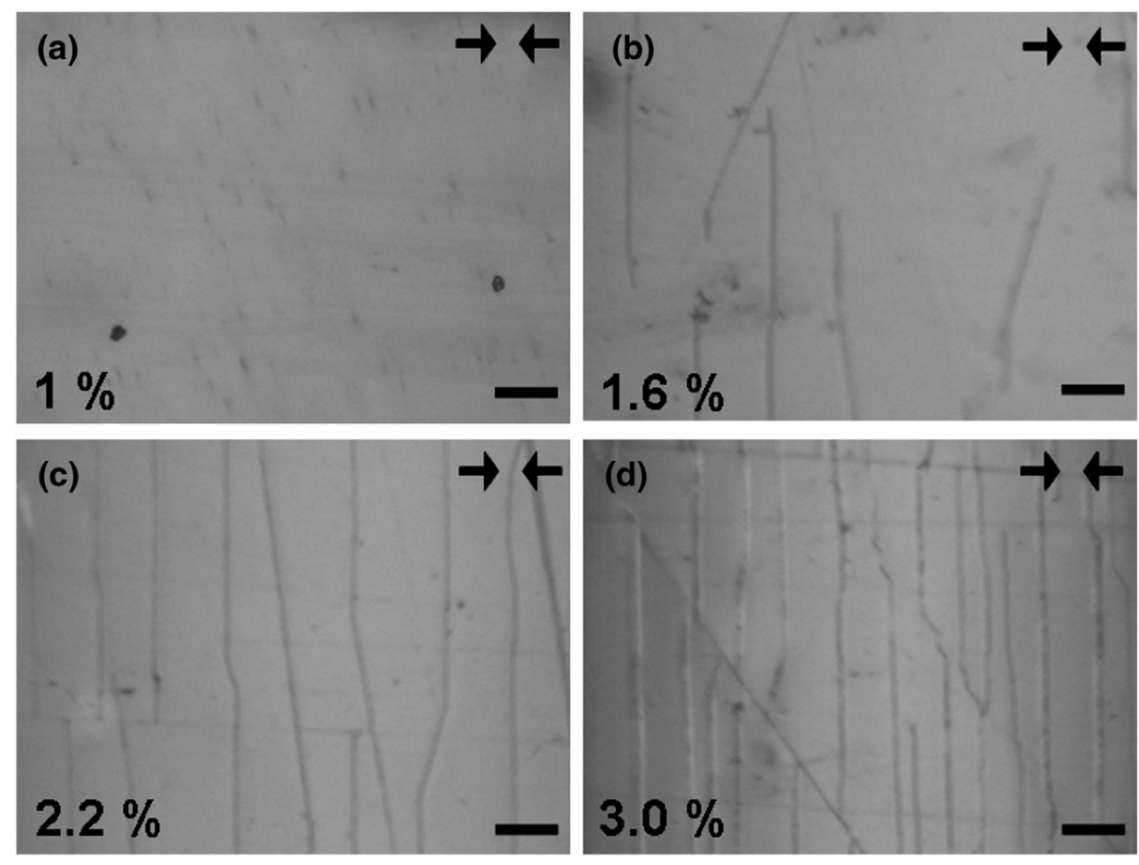

Fig. 6.12 - Typical optical micrographs showing crack initiation and propagation for a $\mathrm{ZnO}$ film, $75 \mathrm{~nm}$ thick, sputtered on a PET substrate under controlled compression conditions up to $3 \%$ strain. Arrows indicate the compression direction. Scale bar is equal to $25 \mu \mathrm{m}$.

When the externally applied strain reaches a critical value, the onset of straight cracks with a general direction oriented perpendicular to the compression direction were observed (Fig. 6.12b). It was observed that initially the length of the cracks does not span the whole width of the sample. However as the externally applied strain increases (Fig. $6.12 \mathrm{c})$, the length of the cracks increases, due to steady state propagation through the $\mathrm{ZnO}$ thickness, and extends to the whole length of the sample. Further increase of applied strain, as depicted in Fig. 6.12d, results in an increased number of cracks and an associated decrease in spacing between cracks. Observation of crack morphology using SEM, as shown in Fig. 6.13, reveals a typical inter-granular cohesive failure mechanism. 


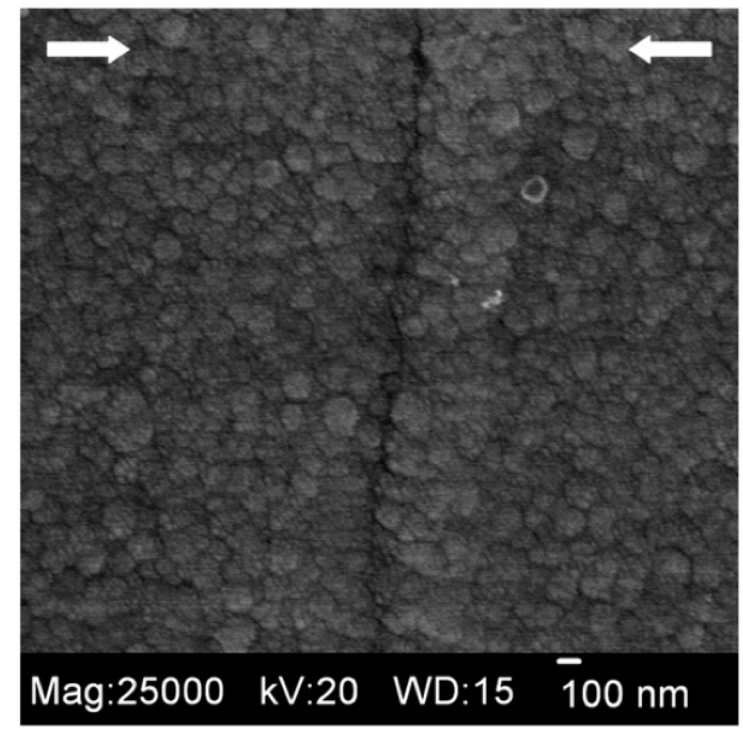

Fig. 6.13 - SEM image of crack morphology for a $650 \mathrm{~nm}$ thick $\mathrm{ZnO}$ film sputtered on PET. Arrows indicate compression direction.

Neighboring islands with sizes ranging from 25 to $100 \mathrm{~nm}$ are observed to separate at their boundaries upon the action of the applied force. The critical strain at which cracks initiate on the $\mathrm{ZnO}$ surface is a very important parameter when designing flexible devices. The critical onset strain for cracking indicates the maximum strain at which the device can be flexed, bent or stretched and still remain functional. Crack onset strain (COS) values for $\mathrm{ZnO}$ films with thicknesses ranging from 75 up to $1050 \mathrm{~nm}$ on PET and PEN substrates (Fig. 6.14). 


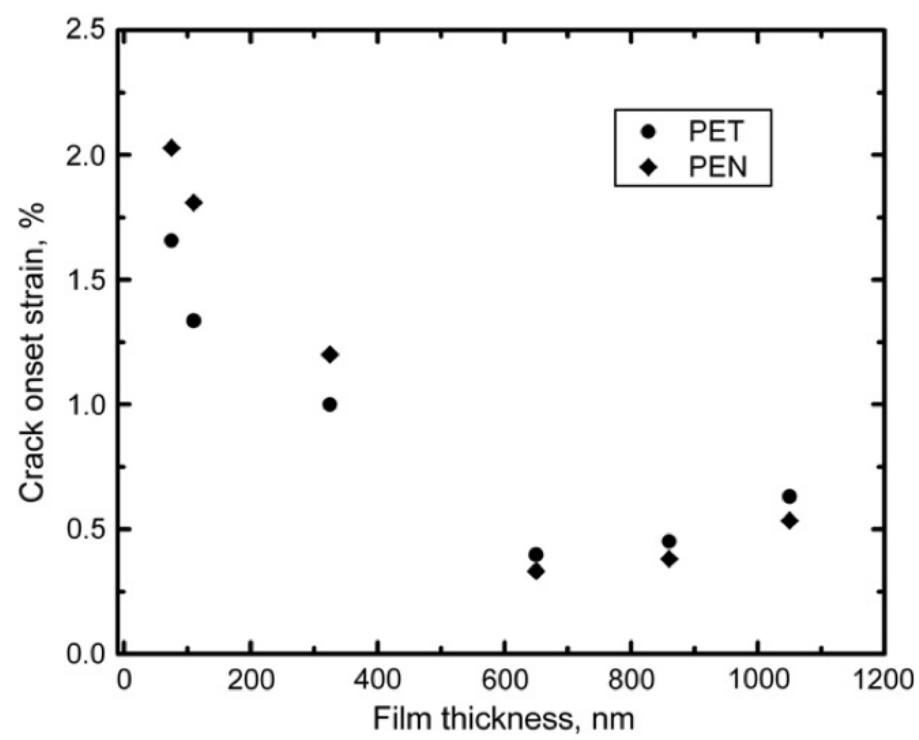

Fig. 6.14 - Graph showing in situ optically measured crack-onset strain versus film thickness for ZnO films sputtered on PET and PEN substrates.

Higher crack onset strains were observed for the thinner samples for both PET and PEN. In particular, as thickness increases the crack onset strain decreases until a thickness of $650 \mathrm{~nm}$ is reached. Above $650 \mathrm{~nm}$ the lowest crack onset strains were observed with a slight increase in onset strain as thickness further increases. This can be attributed to a compressive-tensile-compressive residual stress evolution as thickness increases during sputtering. Maximum values, around 2\%, of crack onset strain are observed for the $75 \mathrm{~nm}$ thick films deposited on PEN. These values are comparable with crack onset values for ceramic-based films such as ITO. The strain drops to $0.33 \%$ when the $\mathrm{ZnO}$ film is 650 $\mathrm{nm}$ thick. This decrease in critical strain can be also associated with the increase of the number of nano-sized defects in the coating due to increased thickness. The thinnest films deposited on PEN exhibit higher crack onset strain values, as compared to their PET counterparts, whereas at larger thicknesses the crack onset strain values are similar for both substrates. Since PET is observed to exhibit slightly better adhesion than PEN, the 
stress transferred from the PET substrate to the interface is relieved by cracking at lower strains as compared to PEN.

\subsubsection{Line-Contact Reciprocating Wear Testing}

Rolling-sliding contact tribological experiments were performed to further the investigations regarding the sustained durability and wear resistance of the piezoelectric $\mathrm{ZnO}$ films in energy harvesting devices. A description of the custom-built reciprocating wear tester can be found in Fig. 2.5. The Hertzian contact pressure of the cylinder counterface was calculated to be $16 \mathrm{MPa}$.

Weight measurements were carried out before and after experiments to determine the wear performance of the film during repeated reciprocating rolling-sliding contact. The $\mathrm{ZnO}$ bottom counterface and stainless steel cylindrical counterfaces were both weighed after each experiment.

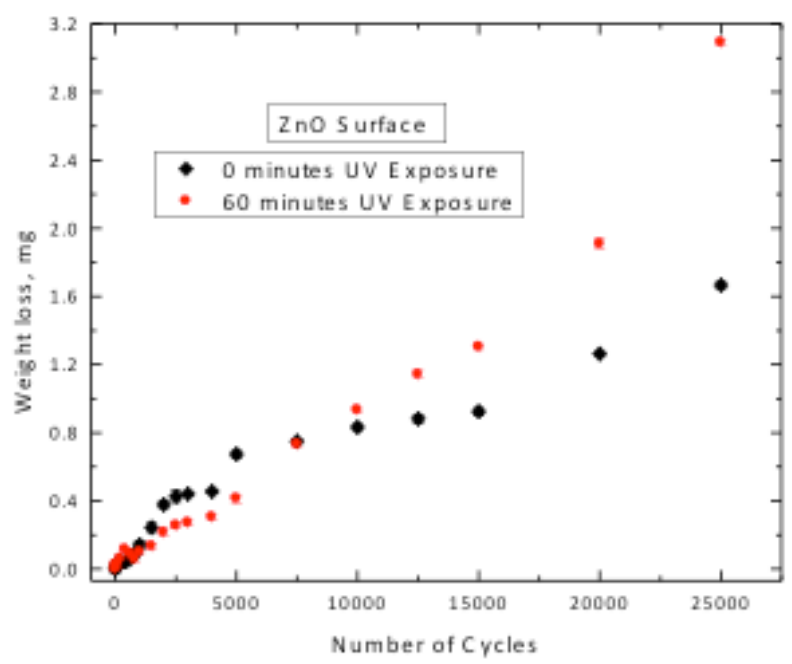

Fig. 6.15 - Weight loss of $\mathrm{ZnO}$ films versus number of reciprocating rolling-sliding cycles. The counterface is a stainless steel cylinder. 
Fig. 6.15 presents the variation of weight loss with the number of reciprocating cycles for native films and after one hour of UV irradiation. Relatively little weight loss was observed for samples below 7000 reciprocating wear cycles. Above 7000 cycles, a significant increase in wear of the UV-exposed $\mathrm{ZnO}$ surface was observed. This can be attributed to an increased adhesion force, due to the UV exposure, coupled with possible surface fatigue, especially above 20,000 reciprocating cycles. The non-UV exposed sample was observed to exhibit slightly increased wear above 7000 cycles. However, the observed wear was not as pronounced as in the UV-exposed counterpart.

The weight gain of the counterface as opposed to the number of reciprocating cycles is shown in Fig. 6.16. This can be attributed to material transfer from the $\mathrm{ZnO}$ samples to the counterface.

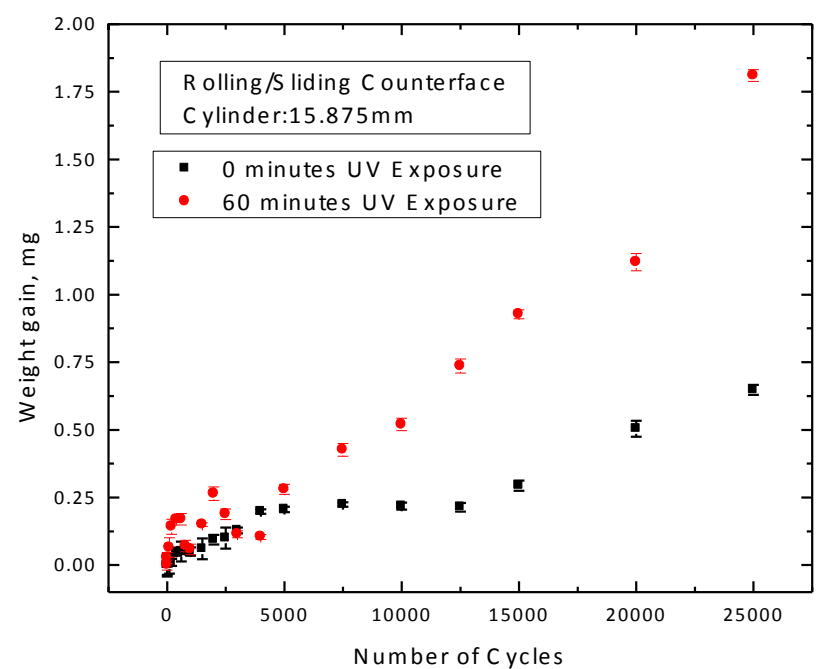

Fig. 6.16 - Weight gain of the rolling stainless-steel counterface versus number of reciprocating cycles. Significant material transfer from the film to the steel cylinder was observed when the $\mathrm{ZnO}$ surface was exposed to UV light. This is consistent with our previous observations 
indicating increased $\mathrm{ZnO}$ surface adhesion upon exposure to UV light. The hydrophobic nature of the native $\mathrm{ZnO}$ films leads to relatively limited material transfer to the rollingsliding counter-body.

$\mathrm{ZnO}$ surfaces were observed using optical microscopy as a means of characterizing wear mechanisms present. Fig. 6.17 shows images of the non-UV exposed surfaces after various numbers of reciprocating cycles.
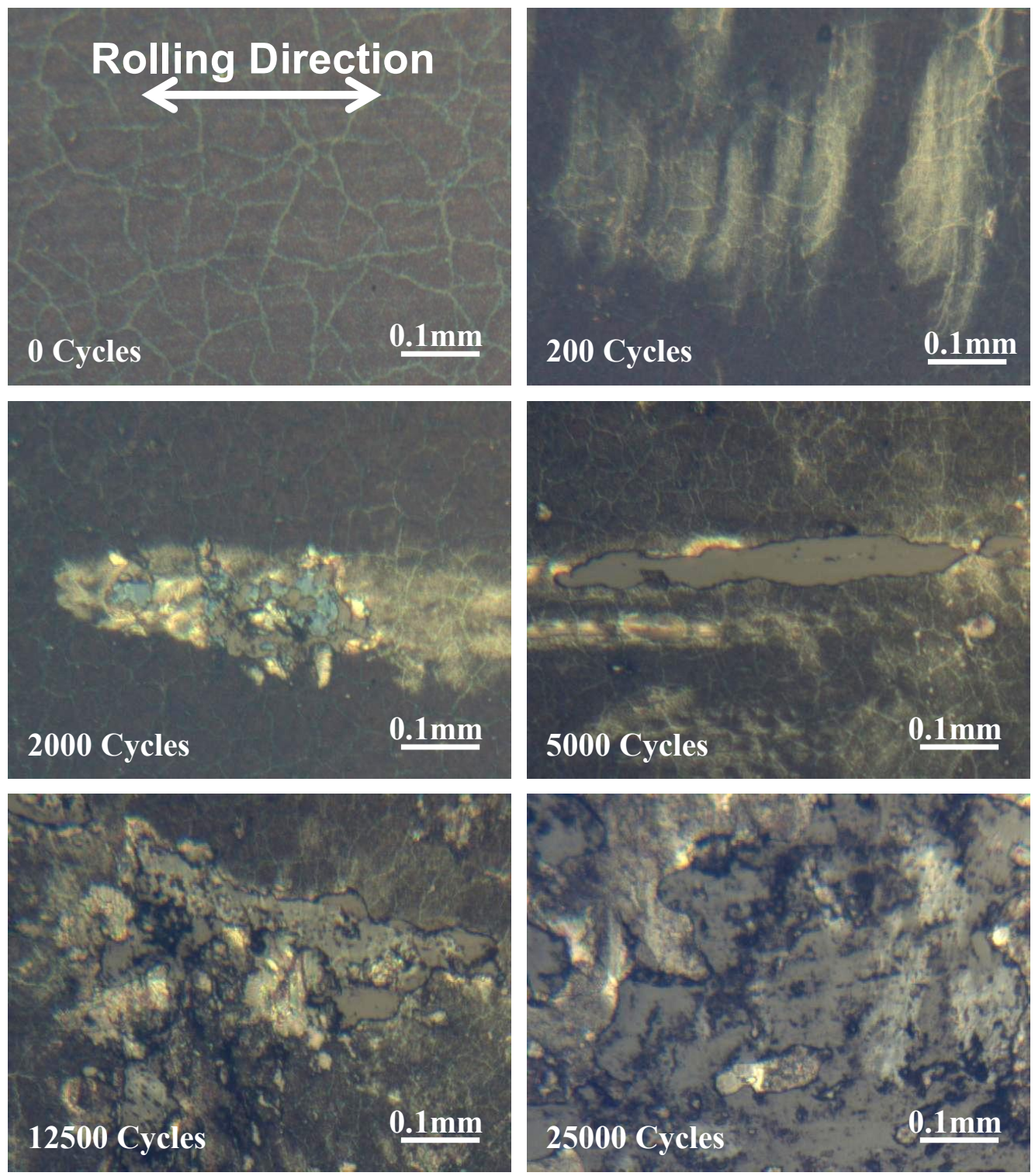

Fig. 6.17 - Optical images of non-UV exposed $\mathrm{ZnO}$ surfaces taken after various amounts of reciprocating 
rolling. The rolling direction is the same for all images.

Fig. 6.18 presents images of the $\mathrm{ZnO}$ films exposed to UV light for 1 hour, after a number of reciprocating cycles.
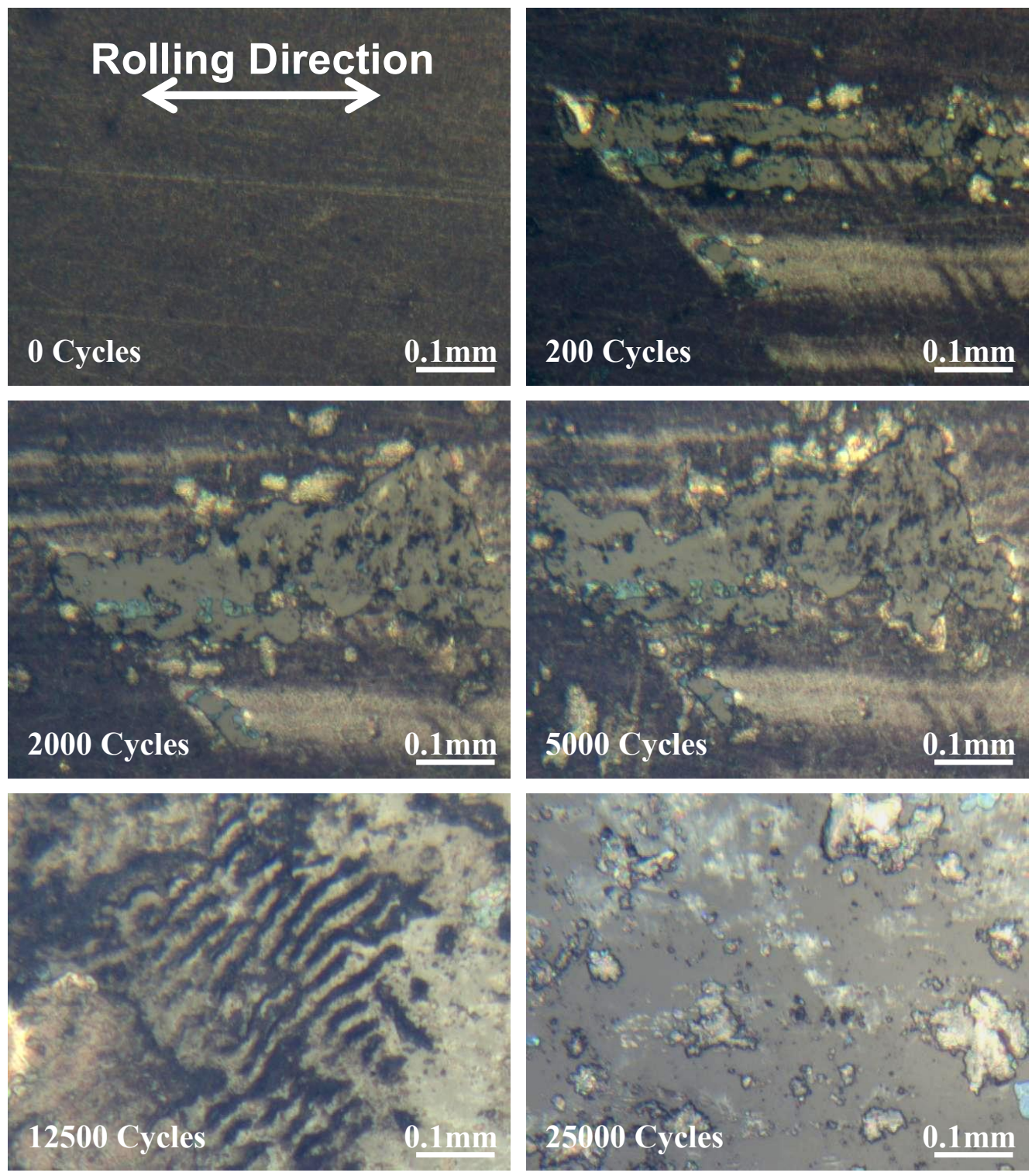

Fig. 6.18 - Optical image of 60 minute UV exposed $\mathrm{ZnO}$ surfaces taken after various amounts of reciprocating rolling cycles. The rolling direction is the same for all images.

In all cases, mixed rolling/sliding tribological mechanisms were observed. Microcrack formation and delamination were observed above 200 cycles in all cases. As the number of cycles increased, these wear mechanisms were increasingly apparent. This indicates 
the significant role that the surface fatigue plays in rolling-sliding tribological

applications. The generated $\mathrm{ZnO}$ debris may also play a significant role, especially in the UV-exposed surface case, as a third body that induces abrasive wear. This debris was generated by the formation of adhesive junctions and their subsequent rupture. The cyclic stress interactions may also be responsible for the abrasion mechanisms observed. Three significant wear mechanisms were observed at relatively high numbers of reciprocating cycles; microcutting, microplowing and microcracking.

The $\mathrm{ZnO}$ material transfer to the cylindrical counter-surface is shown in Fig. 6.19.
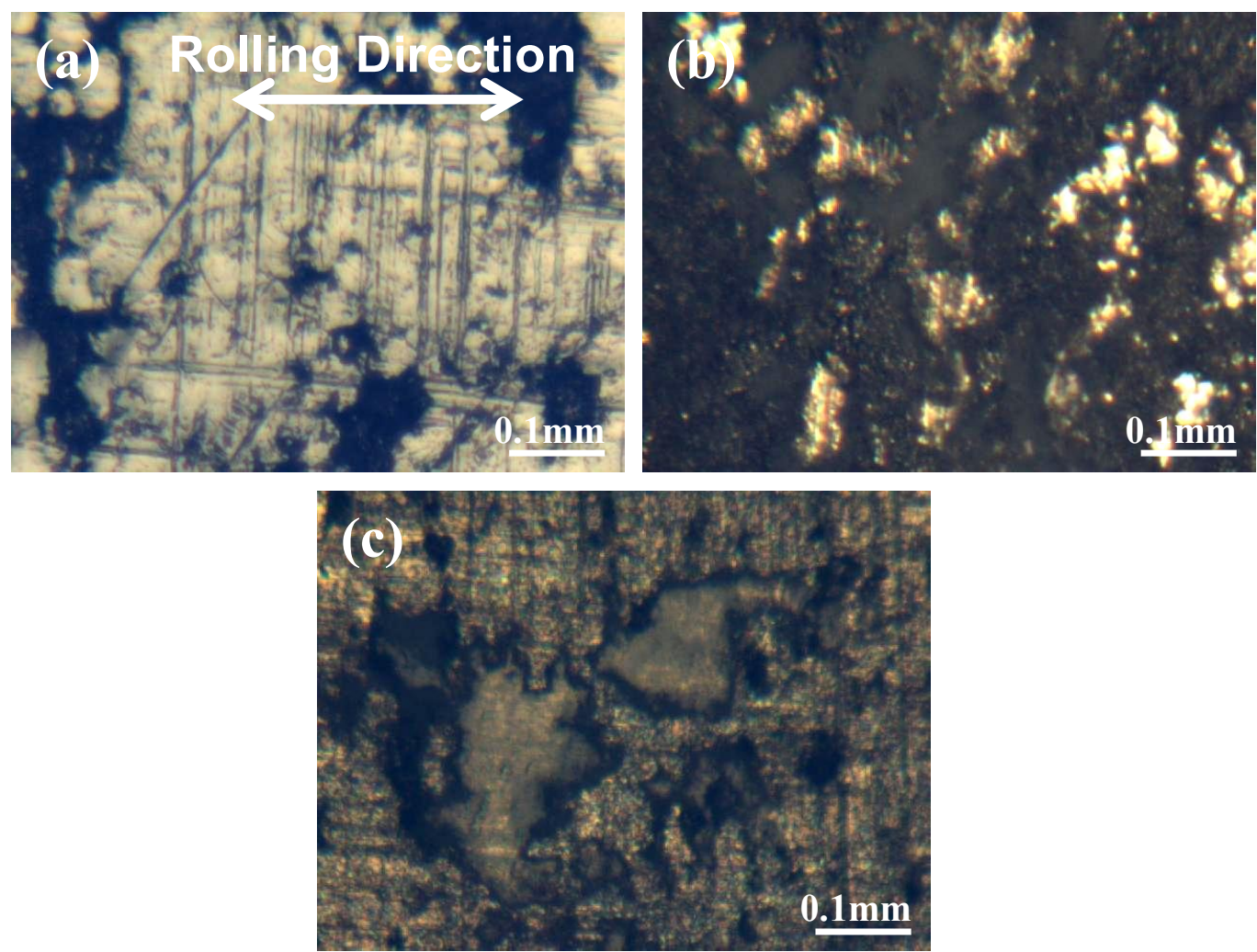

Fig. 6.19 - Optical images of stainless steel cylinder surfaces. A) Initial counterface, b) surface after 25,000 rolling cycles on a non-UV exposed $\mathrm{ZnO}$ surface, c) cylinder surface after 25,000 reciprocating cycles on a 60 minute $\mathrm{UV}$ exposed $\mathrm{ZnO}$ film surface.

The counterface surface was observed to have accumulated debris. This phenomenon is more apparent in the UV-exposed surface case where the whole surface area appears to 
be covered by debris particles.

Furthermore, the rolling/sliding based piezoelectric output of a preliminary device design was investigated using the apparatus depicted in Fig. 2.5. This apparatus was designed to permit in situ electrical resistance monitoring of the tribological pair while contact.
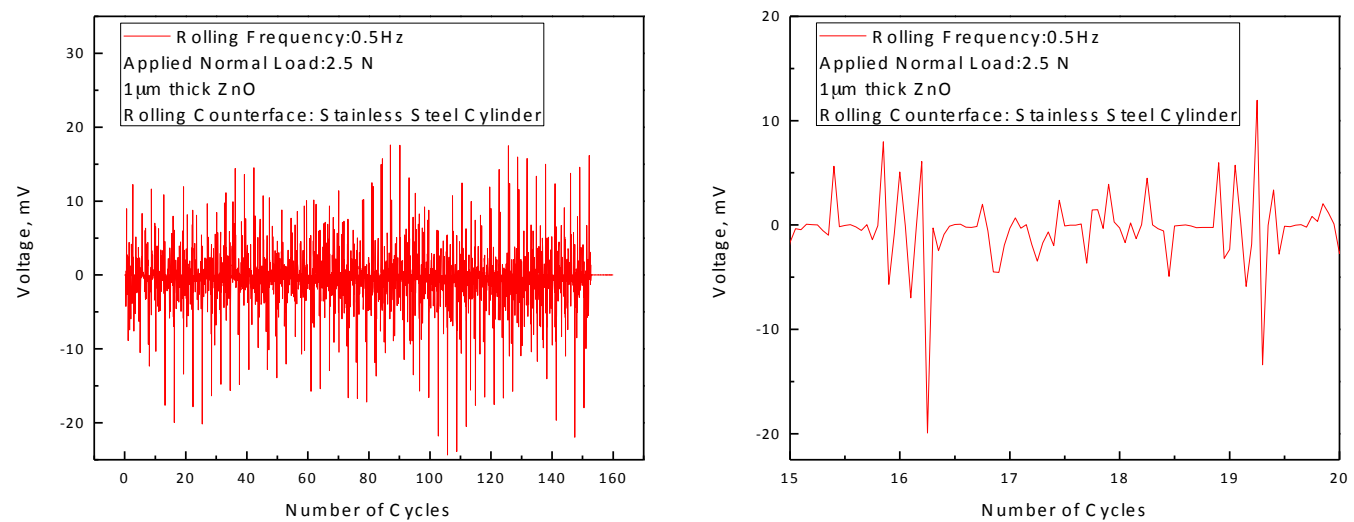

Fig. 6.20 - Voltage response of preliminary device design subjected to rolling for a total of 150 cycles at a frequency of $0.5 \mathrm{~Hz}$. a) All 150 cycles, and b) cycles from 15 to 20.

Figs. 6.20 and 6.21 show preliminary results of the device response versus number of reciprocating cycles. This device energy-harvesting device exhibited a maximum opencircuit voltage output of $24 \mathrm{mV}$, under mixed rolling/sliding conditions for a rolling frequency equal to $0.5 \mathrm{~Hz}$. 

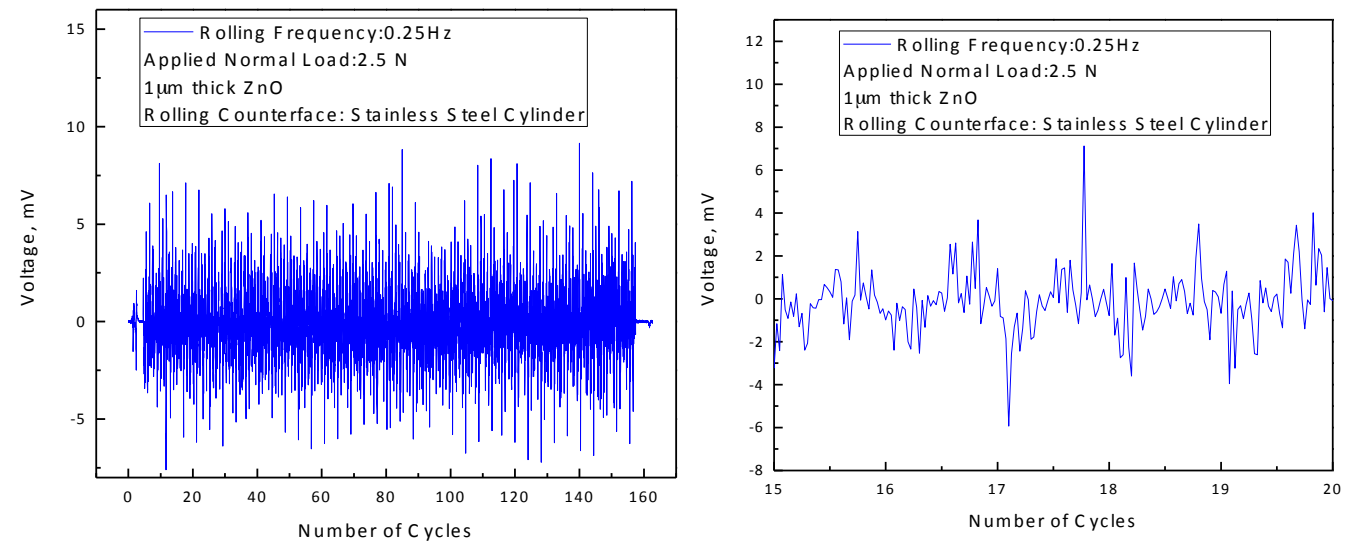

Fig. 6.21 - Voltage response of preliminary device design subjected to rolling for a total of 150 cycles at a rolling frequency of $0.25 \mathrm{~Hz}$. a) All 150 cycles, and b) cycles from 15 to 20.

Significant hysteresis was observed, especially with increased frequency. This can be attributed to the contact conditions established in the tribological interface. When a lower rolling frequency was used, less hysteresis is observed in general. This was attributed to piezoelectric crystals having more time to charge-discharge between contact.

Additionally, the generation of less frictional heating may lead to the reduced hysteresis response. However, in both cases the preliminary devices were able to perform reliably for up to 7500 cycles.

\subsubsection{Point-Contact Reciprocating Wear Testing}

Further rolling-sliding experiments were conducted using a point contact stainless steel ball counterface, in the previously described reciprocating wear tester (Fig. 2.6). Film patterns for this work were created using photolithography and wet chemical etching. Patterns feature alternating parallel lines or squares with equal major dimensions and structure spacing equal to 50 or $100 \mu \mathrm{m}$. 
Contact mode surface profilometry with a $12.5 \mu \mathrm{m}$ stylus was conducted to twodimensionally map the wear scars present several hundred wear cycles. The stylus scanning direction was perpendicular to reciprocating wear direction. Fig. 6.22 presents the wear track profile of an unpatterned sample after 500 cycles of reciprocating combined rolling-sliding contact. Optical micrographs are also shown to visualize the wear mechanisms. The optical image shows parallel linear scratches, which can be attributed to sliding wear. The perpendicular profile of the wear track does not show a significant height change and shows little displacement of the surface material.

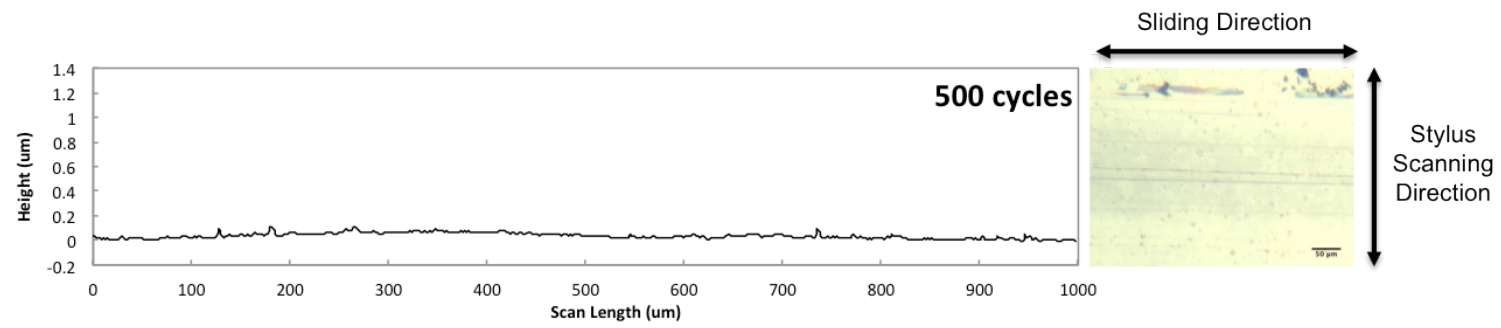

Fig. 6.22 - Typical surface profile of unpatterned $\mathrm{ZnO}$ film after 500 reciprocating cycles with an optical image of the wear track.

Fig. 6.23 presents the wear track profiles and corresponding optical micrographs of 100 $\mu \mathrm{m}$-wide $\mathrm{ZnO}$ lines. Wear debris on the edges of the wear track is visible on both optical images and profiles for all values of reciprocating cycles. The amount of debris increases with reciprocating cycles as expected. 


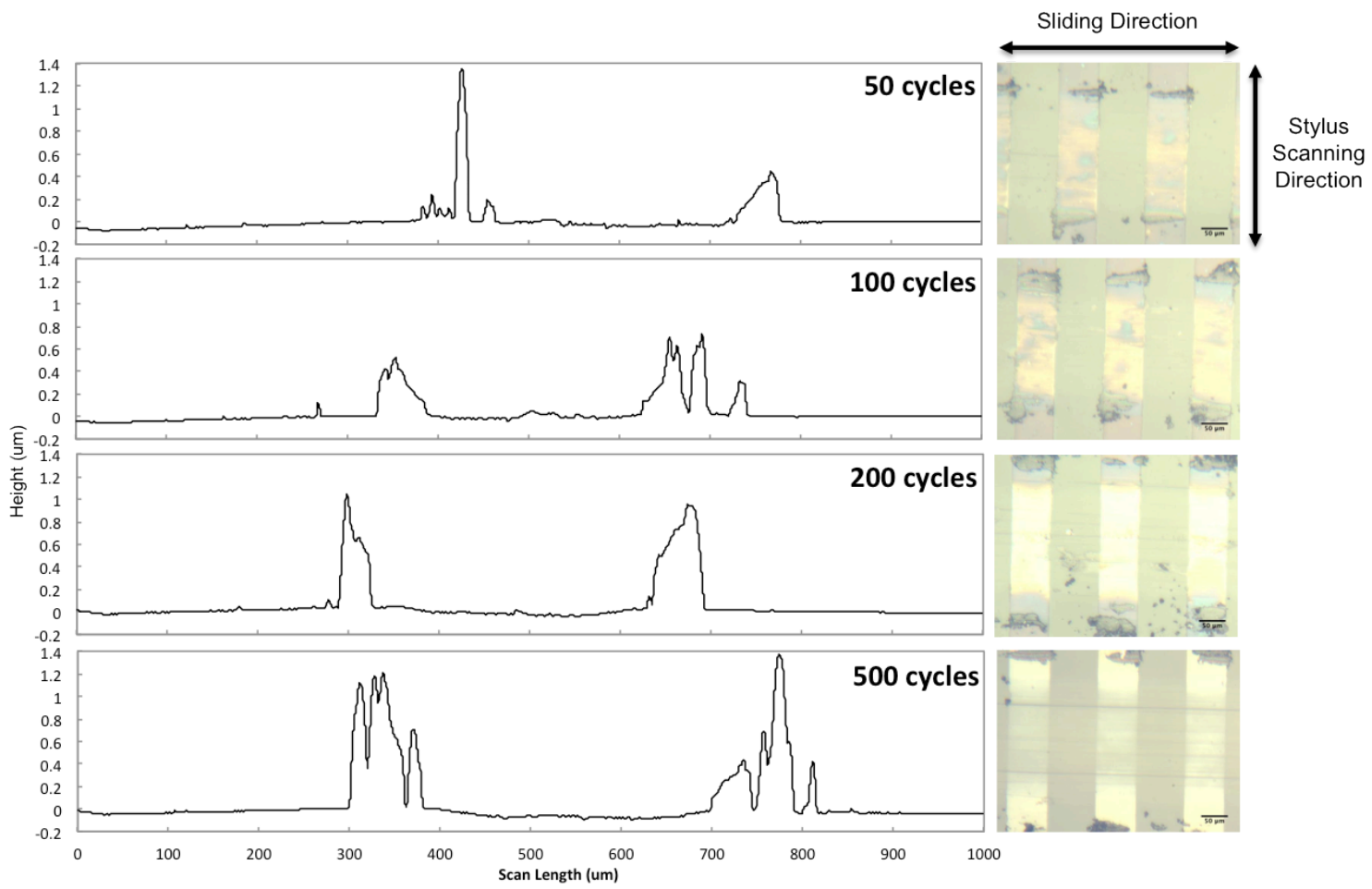

Fig. 6.23 - Typical surface profile of $100-\mu \mathrm{m}$ lines sample after various numbers of reciprocating cycles with optical images of the wear tracks.

Fig. 6.24 shows the wear profiles and optical micrographs for the $100-\mu \mathrm{m}$ squares sample. Significant wear of the cubic $\mathrm{ZnO}$ features is observed after reciprocating cycles greater than 100 cycles. Optical images show this significant wear and reduction in feature height. The image at 500 cycles shows the $\mathrm{ZnO}$ structures centralized in the wear track have reduced by over half their original height. It is important to note the amount of wear debris deposited along the edge of the wear track. Patterned squares closer to the edges of the wear tracks show cohesive failure of the $\mathrm{ZnO}$ structures. 


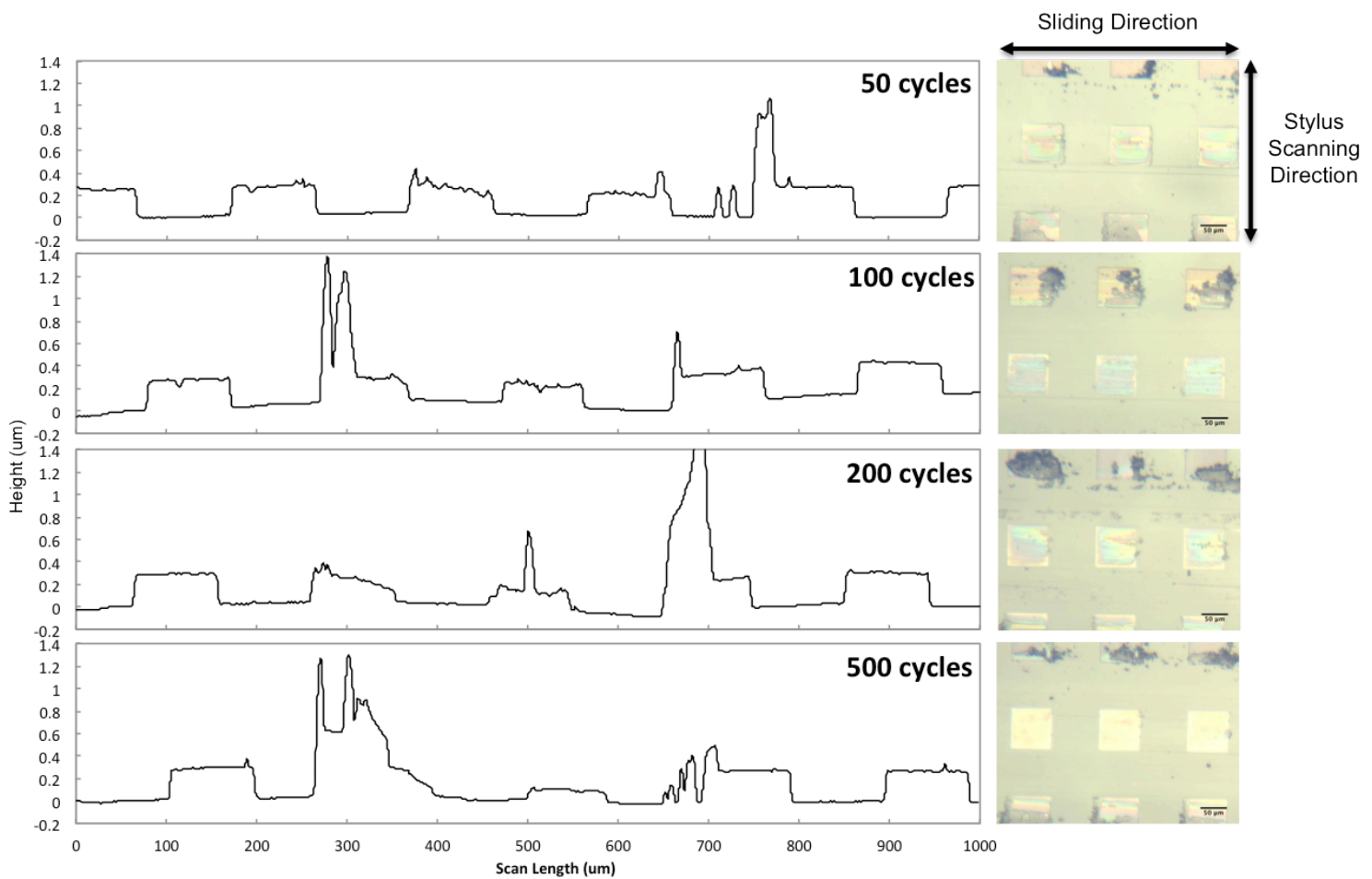

Fig. 6.24 - Typical surface profile of 100- $\mu \mathrm{m}$ squares sample after various numbers of reciprocating cycles with optical images of the wear tracks.

Figs. 6.24 and 6.26 present the stylus and optical analysis of the $50-\mu \mathrm{m}$ lines and $50-\mu \mathrm{m}$ squares samples, respectively. 


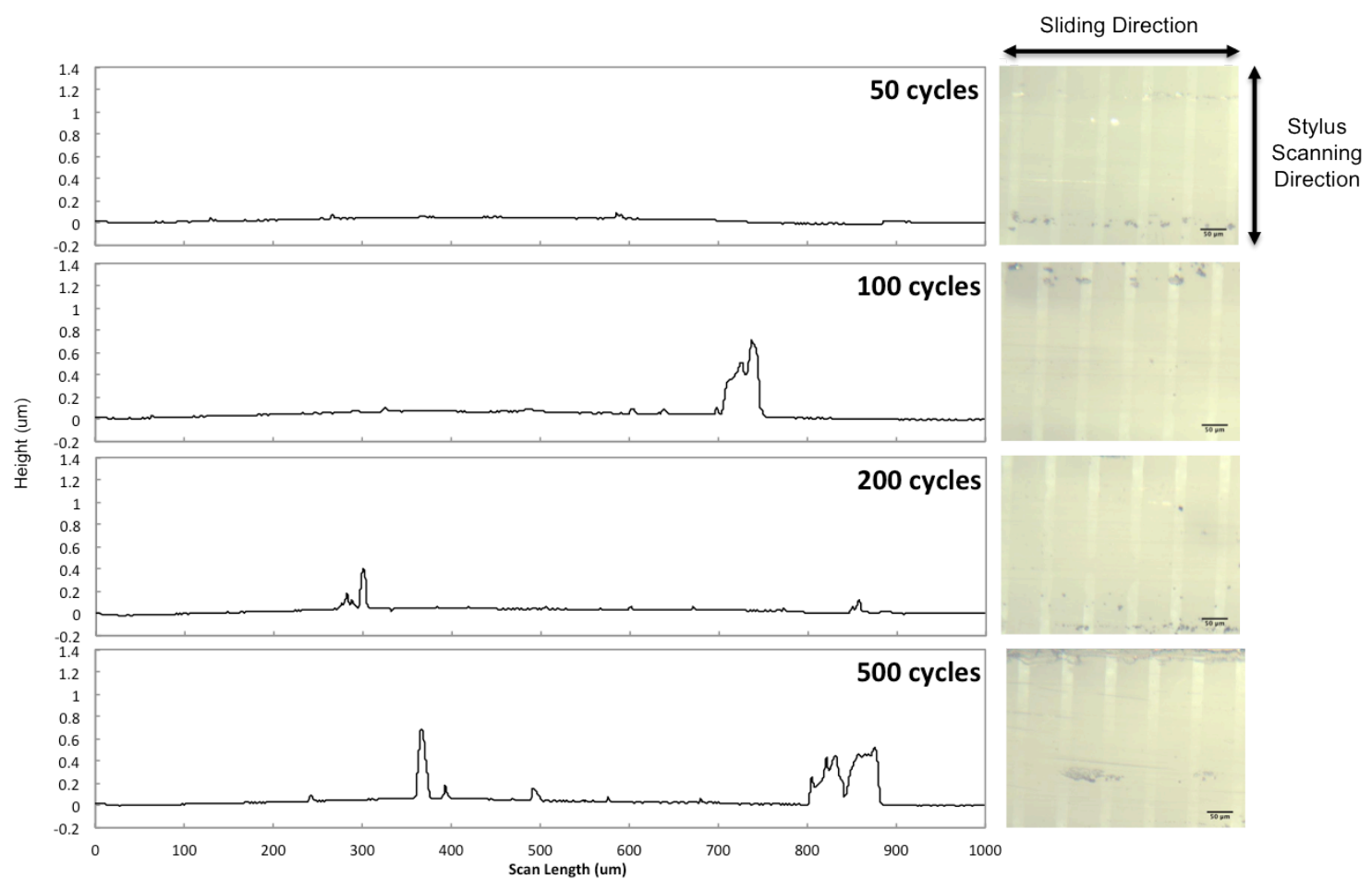

Fig. 6.25 - Typical surface profile of 50- $\mu \mathrm{m}$ lines sample after various numbers of reciprocating cycles with optical images of the wear tracks.

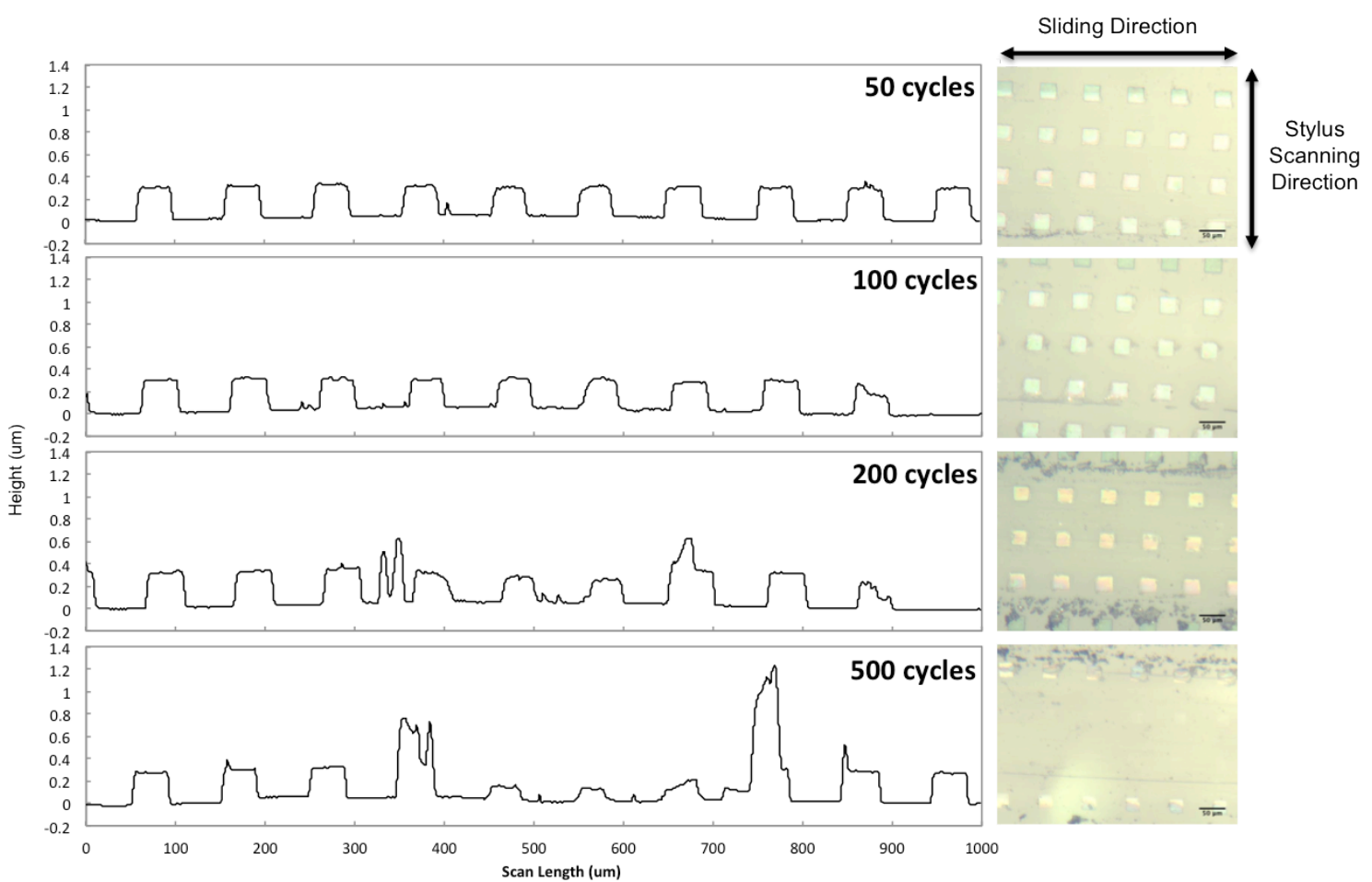

Fig. 6.26 - Typical surface profile of 50- $\mu \mathrm{m}$ squares sample after various numbers of reciprocating cycles with optical images of the wear tracks. 
Fig. 6.27 presents higher magnification optical micrographs of a $100-\mu \mathrm{m}$ line before (left) and after (right) 500 reciprocating wear cycles. Cohesive wear and adhesive failure was observed as the $\mathrm{ZnO}$ structure was completely removed from the center of the wear track for many of the $\mathrm{ZnO}$ lines.
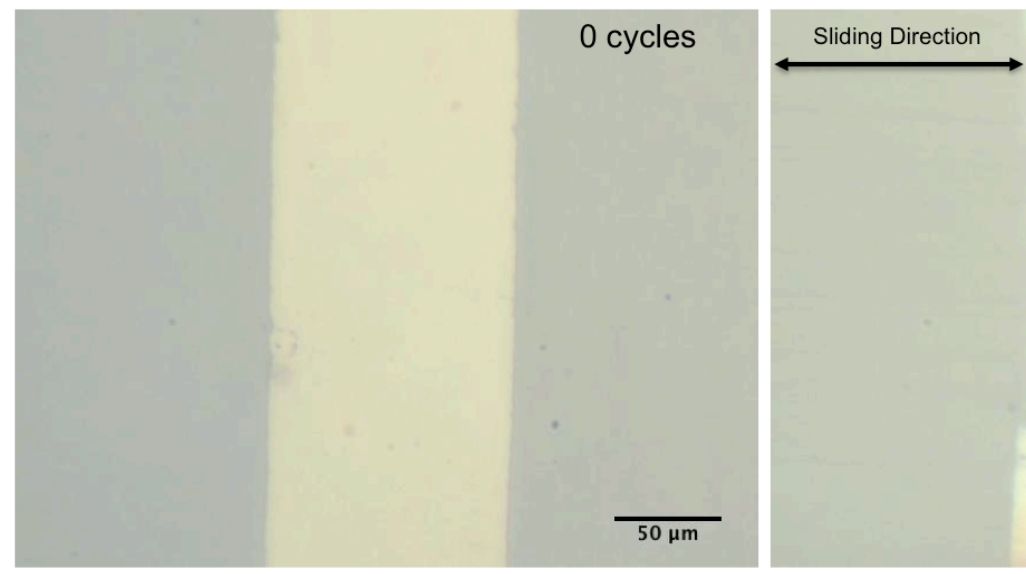

500 cycles

Fig. 6.27 - Optical micrographs of 100- $\mu$ m lines sample before wear testing (left) and after 500 reciprocating wear cycles (right) showing adhesive failure and destruction of the $\mathrm{ZnO}$ line.

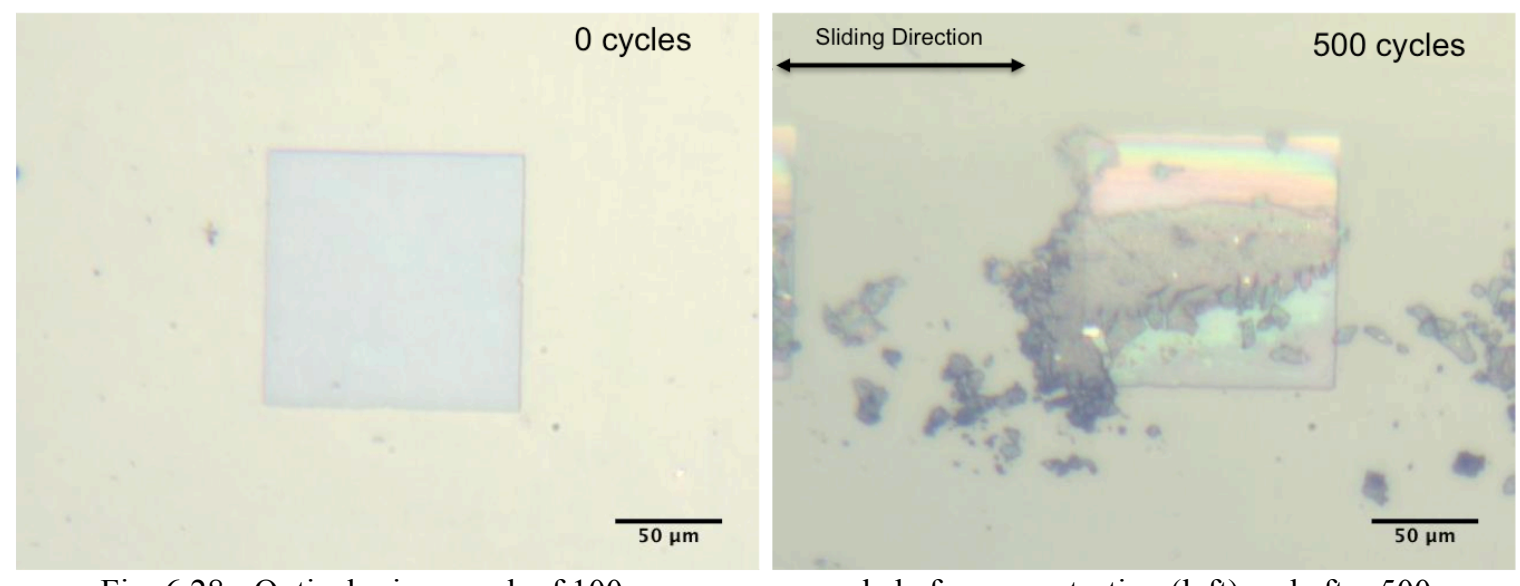

Fig. 6.28 - Optical micrograph of 100- $\mu \mathrm{m}$ square sample before wear testing (left) and after 500 reciprocating wear cycles (right) showing cohesive failure of the $\mathrm{ZnO}$ square structure.

Significant debris deposition can be seen in Fig. 6.28, although weight loss/gain measurements were not significant when performed for this study. This was attributed to the small volume of material available for transfer. 


\subsection{Conclusions}

In this chapter, tribo-mechanical experiments were conducted to characterize the mechanical properties and durability of piezoelectric $\mathrm{ZnO}$ films. Controlled compression and scratch testing experiments with in situ microscopical monitoring were conducted in order to study the mechanical durability of $\mathrm{ZnO}$ coatings that can be used in numerous flexible device applications. It was found that there is a strong relation between crack onset strain, during controlled compression, and coating thickness. Relatively high crack onset values were observed for thinner coatings and in particular the ones that were sputtered on PEN substrates were found to lie around $2 \%$. The failure mechanism was observed to be cohesive failure of the coating in the form of cracks mostly perpendicular to the loading direction with intergranular crack morphology. In addition, two main scratch failure mechanisms were observed during scratch testing. Specifically, coating cracking and buckling delamination were the primary failure mechanisms. Both these mechanisms are thickness dependent. A secondary failure mechanism was observed at high scratch loads with debris from the coating embedded into the soft underlying substrate. An appreciation of the thickness dependent mechanical behavior of $\mathrm{ZnO}$ films may be particularly useful in designing robust devices and appropriate fabrication techniques for flexible devices.

It is also observed during cyclic rolling/sliding contact experiments that the adhesion of the $\mathrm{ZnO}$ film increases with increased UV exposure time, which can be used to control the $\mathrm{ZnO}$ surface friction. Weight measurements indicate limited $\mathrm{ZnO}$ transfer to rolling counterface and relatively limited $\mathrm{ZnO}$ wear below 7500 cycles. Also, the UV exposed $\mathrm{ZnO}$ sample showed significant wear above 7500 cycles compared to the non-UV 
exposed $\mathrm{ZnO}$ sample. Preliminary device design exhibits response to external mixed rolling-sliding stimulus and remains functional for up to 7500 cycles. $\mathrm{ZnO}$ patterns were also exhibited significant wear with point-contact rolling/sliding at 500 reciprocating cycles. The tribological wear mechanisms are of vital importance when designing contact-based energy harvesting devices, and careful considering must be taken when considering $\mathrm{ZnO}$ film thickness, material structure, and contact countersurface. 


\section{General Conclusions}

\subsection{Conclusions}

Initial focus of this work was placed on development of model contact-based devices consisting of flexible electrodes and a piezoelectric zinc oxide film. Devices were successfully fabricated on rigid and flexible platforms, but irreparable damage was observed after only a few loading cycles. Mechanical, tribological, and chemical corrosion investigations were carried out in order to characterize these functional layers using a bottom-up, component-level analysis scheme.

ITO-coated polyester electrodes showed brittle ring cracking, with secondary radial crack formation at advanced loads when loaded in point-contact using a sphero-conical diamond indenter. It was found that the indent radius imprint on the ITO surface increased linearly with increasing load and it was not depended on coating thickness. Also, it was observed that the ring crack spacing, produced at increasing normal loads, was thickness dependent. Increased coating thickness led to larger fragments. Controlled buckling of patterned ITO-coated polyester films showed higher crack onset values for smaller patterns with smoother edges. Sharp edges and poor pattern edge definition was believed to promote cracking at lower strain values. Cohesive cracking and film delamination were observed under both tensile and compressive controlled buckling.

Mechanical stresses combined with chemical corrosion was investigated for ITO-coated PET films using low-concentration acrylic acid and static bending. ITO cracking 
occurred at stresses less than one-quarter of those needed to cause failure without combined chemical corrosion. Tribo-chemical studies of ITO-ITO sliding in dry and wet conditions showed after a threshold of 10,000 sliding cycles, an abrupt increase in film conductivity was observed.

Finally, piezoelectric $\mathrm{ZnO}$ films were investigated and characterized during controlled buckling and repeated wear studies. Controlled buckling experiments showed a strong relation between crack onset strain and coating thickness, similar to the ITO-coated PET experiments. Point and line-contact rolling/sliding experiments was performed in order to characterize tribological wear of functional films. Significant wear was observed for patterned $\mathrm{ZnO}$ films. UV exposure was proven to be a means of controlling the frictional properties of $\mathrm{ZnO}$ surfaces.

This study investigated the functional components of flexible piezoelectric energy harvesting devices for development of standardized mechanical testing protocols not currently available in the field. While a number of energy harvesting devices have been developed on laboratory-scale platforms, the lack of understanding regarding the sustained durability of functional components has limited large-scale manufacture. Development of testing protocols found in this work has advanced fundamental understanding of the complex 'hard-on-soft' component system.

\subsection{Future Work}

\subsubsection{Implementation of Standardized Testing Protocols}

A number of mechanical testing protocols were developed in this work to characterize the properties of the 'hard-on-soft' film composite system. Continued experimentation is 
needed to implement these experiments as industry standards, which is currently lacking in the field of ceramic coatings on flexible substrates.

\subsubsection{Carbon Nanotube/Graphene-based Electrodes}

Carbon-based conductive nanostructures such as CNTs and graphene have shown great

promise in development of more mechanically sound flexible electrodes. CNTs deposited

on flexible electrodes can be much thin than their TCO counterparts, while maintaining the required electrode conductivity. Hybrid electrodes utilizing both TCOs and carbonbased nanostructures can lead to electrodes which are more mechanically reliability under both monotonic and fatigue loading scenarios, while retaining a optically transparent film.

\subsubsection{Direct Writing of Functional Components on Flexible Substrates}

Solution-based direct writing of functional inks has potential to not only reduce material waste by selectively functionalizing regions of thin films, but can produce complex 3-D patterns for improved functionality and mechanical reliability. Inks consisting of conductive nanoparticles or graphene flakes suspended in a polymer matrix can provide a cost-effective, low temperature deposition method for complex and curves substrates, not currently possible with traditional deposition techniques. 


\section{References}

[1] B.Y. Oh, M.C. Jeong, T.H. Moon, W. Lee, J.M. Myoung, J.Y. Hwang, et al., J Appl Phys 99 (2006) 124505.

[2] K. Matsubara, P. Fons, K. Iwata, A. Yamada, K. Sakurai, H. Tampo, et al., Thin Solid Films 341 (2003) 369.

[3] P.F. Garcia, R.S. McLean, M.H. Reilly, G. Nunes Jr, Appl Phys Lett 82 (2003) 1117.

[4] G. Kiriakidis, J. Kortidis, K. Moschovis, D. Dovinos, Sensor Lett 6 (2008) 812.

[5] P. Horvath, S.B. Sadale, M. Suchea, S. Christodoulakis, R. Voicu, C. Tibeica, et al., Sensor Lett 6 (2008) 1.

[6] Z. L. Wang, J. H. Song, Science 2006, 312, 242 - 246.

[7] W. T. Chang, Y. C. Chen, R. C. Lin, C. C. Cheng, K. S. Kao, B. R. Wu, Y. C. Huang. "Design and fabrication of a piezoelectric transducer for wind-power generator" Thin Solid Films 519 (2011) 4687-4693

[8] B. Bhushan, M. Nosonovsky. "Green tribology: principles, research areas and challenges" Phil. Trans. R. Soc. A 368 (2010) 4677-4694

[9] S.Y. Chang, Y.C. Hsiao, Y.C. Huang, Surf Coat Technol 202 (2008) 5416.

[10] J. W. Xu, W. W. Shao, F. R. Kong, Z. H. Feng. "Right-angle piezoelectric cantilever with improved energy harvesting efficiency" Appl. Phys. Lett. 96 (2010) 152904-3

[11] A. Kumar, H. Gullapalli, K. Balakrishnan, A. Botello-Mendez, R. Vajtai, M. Terrones, P. M. Ajayan. "Flexible $\mathrm{ZnO}$-cellulose nanocomposite for multisource energy conversion" Small 7 (2011) 2173-2178

[12] T. Yamamoto, T. Shiosaki, A. Kawabata. "Characterization of $\mathrm{ZnO}$ piezoelectric films prepared by rf planar-magnetron sputtering” J. Appl. Phys. 51 (1980) 3113-312

[13] C. Ye, T. Tamagawa, D. L. Polla. "Experimental studies on primary and secondary pyroelectric effects in $\mathrm{Pb}(\mathrm{ZrxTi1}-\mathrm{x}) \mathrm{O} 3, \mathrm{PbTiO} 3$, and $\mathrm{ZnO}$ thin films" J. Appl. Phys. 70 (1991) 5538-5544

[14] S. V. Prasad, S. D. Walck, J. S. Zabinski. "Microstructural evolution in lubricious ZnO films grown by pulsed laser deposition” Thin Solid Films 360 (2000) 107-117

[15] S. Roundy, P.K. Wright, J. Rabaey, Comput. Commun. 26 (2003) 1131.

[16] D. Kim, S. Hong, D. Li, H.S. Roh, G. Ahn, J. Kim, M. Park, J. Hong, T.-h. Sung, K. No, RSC Advances 3 (2013) 3194.

[17] K. Allen, In: G.P. Crawford (ed.) Flexible flat panel displays, John Wiley \& Sons, 2005, p. 495.

[18] M.C. Choi, Y. Kim, C.S. Ha, Prog. Polym. Sci. 33 (2008) 581.

[19] C. Guillen, J. Herrero, Thin Solid Films 520 (2011) 1.

[20] D.H. Kim, M.R. Park, H.J. Lee, G.H. Lee, Applied Surface Science 253 (2006) 409.

[21] Y.Z. You, Y.S. Kim, D.H. Choi, H.S. Jang, J.H. Lee, D. Kim, Materials Chemistry and Physics 107 (2008) 444.

[22] W.A. MacDonald, M.K. Looney, D. MacKerron, R. Eveson, R. Adam, K. Hashimoto, K. Rakos, Journal of the SID 15/12 (2007) 1015.

[23] S. Logothetidis, Rev.Adv.Mater.Sci. 10 (2005) 387.

[24] B.A. MacDonald, K. Rollins, D. MacKerron, K. Rakos, R. Eveson, K. Hashimoto, B. Rustin, In: G.P. Crawford (ed.) Flexible Flat Panel Displays, John Wiley \& Sons, 2005, p. 11.

[25] T. Ma, B. Bhushan, Journal of Applied Polymer 88 (2003) 2082. 
[26] E. Schwartz, Cornell University MSE 542(2006) 1.

[27] D.C. Paine, H.-Y. Yeom, B. Yaglioglu, in: G.P. Crawford (Ed.), Flexible Flat Panel Displays, John Wiley \& Sons, New York, 2005, p. 79, (Chapter 5).

[28] C. Nunes de Carvalho, G. Lavareda, E. Fortunato, A. Amaral, Thin Solid Films 427 (2003) 215.

[29] C. Coutal, A. Azema, J.C. Roustan, Thin Solid Films 288 (1996) 248.

[30] D.C. Paine, H.Y. Yeom, B. Yaglioglu, In: G.P. Crawford (ed.) Flexible Flat Panel Displays, John Wiley \& Sons, 2005, p. 79.

[31] XX

[32] H.S. Kwok, X.W. Sun, D.H. Kim, Thin Solid Films 335 (1998) 299.

[33] S.O. Yoon, H.J. Jung, K.H. Yoon, Solid State Communications 64 (1987) 617.

[34] H. Ma, J.S. Cho, C.H. Park, Surface and Coatings Technology 153 (2002) 131.

[35] Q. Cao, J.A. Rogers, ACS Nano 2 (2008) 1266.

[36] J.L. Blackbum, T.M. Barnes, M.C. Beard, Y.H. Kim, R.C. Tenent, T.J. McDonald, B. To, T.J. Coutts, M.J. Heben, Adv. Mater. 21 (2009) 29.

[37] E.M. Doherty, S. De, P.E. Lyons, A. Shmeliov, P.N. Nirmalraj, V. Scardaci, J. Joimel, W.J. Blau, J.J. Boland, J.N. Coleman, Carbon 47 (2009) 2466.

[38] K.A. Sierros, D.S. Hecht, D.A. Banerjee, N.J. Morris, L. Hu, G.C. Irvin, R.S. Lee, D.R. Cairns, Thin Solid Films 518 (2010) 6977.

[39] T. Yamamura, M. Kitamura, K. Kuribayahi, Y. Arakawa, S. Takeuchi, MEMS 20 (2007) 739.

[40] Z.L. Wang, "Zinc Oxide nanostructures: growth, properties and applications" Journal of Physics: Condensed Matter 16 (2004) R829-R858

[41] Z.L. Wang, W. Wu, "Nanotechnology-Enabled Energy Harvesting for Self-Powered Micro-/Nanosystems" Angewandte Chem. Int. Ed. 51 (2012) 2-24

[42] Z.L. Wang, "Ten years' venturing in $\mathrm{ZnO}$ nanostructures: from discovery to scientific understanding and to technology applications" Chinese Science Bulletin (2009)

[43] S. Xu, Y. Wei, J. Liu, R. Yang, and Z. L. Wang, "Integrated Multilayer Nanogenerator Fabricated Using Paired Nanotip-to-Nanowire Brushes", Nano Letters 8 (2008) 4027-4032

[44] R. Yang, Y. Qin, L. Dai and Z. L. Wang, "Power generation with laterally-packaged piezoelectric fine wires", Nature Nanotechnology, 4 (2009) 34-39

[45] G. Zhu, R. Yang, S. Wang, and Z. L. Wang, "Flexible High-Output Nanogenerator Based on Lateral ZnO Nanowire Array", Nano Lett.,Supplementary Information, Live video, 10 (2010) 3151-3155

[46] S. Xu, Y. Qin, C. Xu, Y. Wei, R. Yang and Z. L. Wang "Self-powered nanowire devices ", Nat. Nanotechnol., 5 (2010) 366-373

[47] C. E. Chang, V. H. Tran, J. B. Wang, Y. K. Fuh, L. W. Lin, Nano Lett. 10 (2010) $726-731$

[48] Y. Hu, Y. Zhang, C. Xu, G. Zhu, and Z. L. Wang, "High-Output Nanogenerator by Rational Unipolar Assembly of Conical Nanowires and Its Application for Driving a Small Liquid Crystal Display " Nano Lett.,Supplementary Information ,Live Video 1 Live Video 2 
[49] Y. Hu , C. Xu , Y. Zhang, L. Lin, R. L. Snyder, Z. L. Wang, “A Nanogenerator for Energy Harvesting from a Rotating Tire and its Application as a Self-Powered Pressure/Speed Sensor", Adv. Matter 2011, Online

[50] M.-Y. Choi, D. Choi, M.-J. Jin, I. Kim, S.-H. Kim, J.-Y. Choi, S. Y. Lee, J.M. Kim, S.-W. Kim, "Mechanically Powered Transparent Flexible Charge-Generating Nanodevices with Piezoelectric ZnO Nanorods" Advanced Materials 21 (2009) 21852189

[51] A. Umar, Y.B. Hahn, "Growth of aligned $\mathrm{ZnO}$ nanorods and nanopencils on $\mathrm{ZnO} / \mathrm{Si}$ in aqueous solution: growth mechanism and structural and optical properties" Nanotechnology 18 (2007) 115603

[52] Y.Hu, L. Lin, Y. Zhang, Z.L. Wang, "Replacing a battery by a nanogenerator with 20 V output" Adv. Mater., (2011) 1-5

[53] J.H. Koo, J. Seo, T. Lee, "Nanomaterials on flexible substrates to explore innovation function: From energy harvesting to bio-integrated electronics" Thin Solid Films, 524 (2012) 1-19

[54] M. Suchea, S. Christoulakis, K. Moschovis, N. Katsarakis, G. Kiriakidis, Thin Solid Films 515 (2006) 551.

[55] D.G. Baik, S.M. Cho, Thin Solid Films 354 (1999) 227.

[56] M. Lee, C.-Y. Chen, S. Wang, S.N. Cha, Y.J. Park, J.M. Kim, L.-J. Chou, Z.L. Wang, Adv. Mater. 24 (2012) 1759.

[57] S.V. Prasad, J.S. Zabinski, Wear 203-204 (1997) 498.

[58] Z. Chai, X. Lu, D. He, Surf. Coat. Technol. 207 (2012) 361.

[59] T.-H. Fang, S.-R. Jian, D.-S. Chuu, J. Phys. D 36 (2003) 878

[60] S. Xu, C. Lao, B. Weintraub, Z.L. Wang, "Density-controlled growth of aligned $\mathrm{ZnO}$ nanowire arrays by seedless chemical approach on smooth surfaces" Journal of Material Research 23(8) (2008) 2072- 2077

[61] D. Bao, H. Gu, A. Kuang, Thin Solid Films 312 (1998) 37.

[62] Y.-S. Kim, W.-P. Tai, S.-J. Shu, Thin Solid Films 491 (2005) 153.

[63] L.-Y. Lin, D.-E. Kim, Thin Solid Films 517 (2009) 1690.

[64] M. Ohyama, H. Kozuka, T. Yoko, Thin Solid Films 306 (1997) 78.

[65] L. Znaidi, Mater. Sci. Eng. B Solid 174 (2010) 18.

[66] X. Feng, L. Feng, M. Jin, J. Zhai, L. Jiang, D. Zhu, J. Am. Chem. Soc. 126 (2004) 62

[67] H. Liu, L. Feng, J. Zhai, L. Jiang, D. Zhu, Langmuir 20 (2004) 5659.

[68] R.-D. Sun, A. Nakajima, A. Fujishima, T. Watanabe, K. Hashimoto, J. Phys. Chem. B 105 (2001) 1984.

[69] P. Horváth, G. Kiriakidis, P.M. Nagy, S. Christoulakis, Proceedings of the 2nd IEEE International Conference on Nano/Micro Engineered and Molecular Systems, IEEE NEMS, 2007, p. 421

[70] L. Vayssieres, K. Keis, S.-E. Lindquist, A. Hagfeldt, "Purpose-Built Anisotropic Metal Oxide Material: 3D Highly Oriented Microrod Array of ZnO" Journal of Physical Chemistry B 105 (2001) 3350-3352

[71] L. Vayssieres, K. Keis, A. Hagfeldt, S.-E. Lindquist, "Three-Dimensional Array of Highly Oriented Crystalline ZnO Microtubes" Chemistry of Materials 13(12) (2001) 4395-4398 
[72] L. Vayssieres, "Growth of Arrayed Nanorods and Nanowires of $\mathrm{ZnO}$ from Aqueous Solutions" Advanced Materials 15(5) (2003) 464-466

[73] K. Govender, D.S. Boyle, P.B. Kenway, P. O'Brian, "Understand the factors that govern the deposition and morphology of thin films of $\mathrm{ZnO}$ from aqueous solution" Journal of Materials Chemistry 14 (2004) 2575-2591

[74] M. Guo, P. Diao, S. Cai, "Hydrothermal growth of well-aligned ZnO nanorods arrays: Dependence of morphology and alignment ordering upon preparing conditions" Journal of Solid State Chemistry 178 (2005) 1864-1873

[75] S. Muhamad, N.F.A. Rahman, S.A. Bakar, M.H. Mamat, M. Rusop, "Controllable Density of Aligned Zinc Oxide Nanorod Arrays by Molarities Variation" IEEE Symposium on Humanities (2012) 1295-1297

[76] Y. Tak, K. Yong, "Controlled Growth of Well-Aligned ZnO Nanorod Array Using a Novel Solution Method” Journal of Physical Chemistry B 109 (2005) 19263-19269

[77] Q. Li, V. Kumar, Y. Li, H. Zhang, T.J. Marks, R.P.H. Chang, "Fabrication of ZnO Nanorods and Nanotubes in Aqueous Solutions" Chemistry of Materials 17 (2005) 1001-1006

[80] Q. Ahsanulhaq, A. Umar, Y.B. Hahn, "Growth of aligned $\mathrm{ZnO}$ nanorods and nanopencils on $\mathrm{ZnO} / \mathrm{Si}$ in aqueous solution: growth mechanism and structural and optical properties" Nanotechnology 18 (2007) 115603

[81] J. Song, S. Lim, "Effect of Seed Layer on the Growth of ZnO Nanorods" Journal of Physical Chemistry C 111 (2007) 596-600

[82] Z. Chen, L. Gao, "A facile route to $\mathrm{ZnO}$ nanorods arrays using wet chemical method" Journal of Crystal Growth 293 (2009) 522-527

[83] X.D. Wang, J.H. Song, J. Liu and Z.L. Wang, "Direct-Current Nanogenerator Driven by Ultrasonic Waves", Science, 316 (2007) 102-105

[84] S. Xu, Y. Wei, J. Liu, R. Yang, and Z. L. Wang, "Integrated Multilayer Nanogenerator Fabricated Using Paired Nanotip-to-Nanowire Brushes", Nano Letters 8 (2008) 4027-4032

[85] R. Yang, Y. Qin, L. Dai and Z. L. Wang, "Power generation with laterally-packaged piezoelectric fine wires", Nature Nanotechnology, 4 (2009) 34-39

[86] G.-W. She, X.-H. Zhang, W.-S. Shi, X. Fan, J.C. Chang, C.-S. Lee, S.-T. Lee, C.-H. Liu, "Controlled synthesis of oriented single-crystal $\mathrm{ZnO}$ nanotube arrays on transparent conductive substrates" Applied Physics Letters 92 (2008) 053111

[87] D.R. Cairns, R.P. Witte II, D.K. Sparacin, S.M. Sachsman, D.C. Paine, R.R. Newton, G.P. Crawford, Strain-dependent electrical resistance of tin-doped indium oxide on polymer substrates, Applied Physics Letters 76 (2000) 1425.

[88] P.C.P. Bouten, Failure test for brittle conductive layers on flexible dis- play substrates, Eurodisplay 2002, Society of Information Display (2002) 313-316.

[89] Z.Chen,B.Cotterell,W.Wang,E.Guenther,S.-J.Chua,A mechanical assessment of flexible optoelectronic devices, Thin Solid Films 394 (2001) 202-206.

[90] Z. Chen, B. Cotterell, W. Wang, The fracture of brittle thin films on compliant substrates in flexible displays, Engineering Fracture Mechanics 69 (2002) 597-603.

[91] K.A. Sierros, J.S. Abell, S.N. Kukureka, Biaxial testing of thin functional structures used in flexible display and touch-screen applications, Eurodisplay 2005, Society of Information Display (2005) 229-232. 
[92] D. R. Cairns, G. P. Crawford. "Electromechanical properties of transparent conducting substrates for flexible electronic displays" P. IEE 93 (2005) 1451

[93] K. A. Sierros, S. N. Kukureka. "Mechanical integrity of touch-screen components" J. Soc. Inf. Display 17 (2009) 947

[94] K. A. Sierros, S. N. Kukureka. "Tribological investigation of thin polyester substrates for displays" Wear 263 (2007) 992

[95] K. A. Sierros, D. R. Cairns, D. S. Hecht, C. Ladous, R. Lee, C. Niu. "Highly durable transparent carbon nanotube films for flexible displays and touch-screens" SID Symp. Digest 41 (2010) 1942

[96] K. A. Sierros, D. S. Hecht, D. A. Banerjee, N. J. Morris, L. Hu, G. C. Irvin, R. S. Lee, D. R. Cairns. "Durable transparent carbon nanotube films for flexible device components" Thin Solid Films 518 (2010) 6977

[97] D. S. Hecht, K. A. Sierros, R. S. Lee, C. Ladous, C. Niu, D. A. Banerjee, D. R. Cairns. "Transparent conductive carbon nanotube films directly coated onto flexible and rigid polycarbonate" J. Soc. Inf. Display 19 (2011) 157

[98] S.W. Gaarenstroom, M.P. Balogh, M.C. Militello, R.A. Waldo, C.A. Wong, N.A. Kelly, T.L. Gibson, M.D. Kundrat, Characterization of indium-tin-oxide films with improved corrosion resistance, Surface and Interface Analysis 37 (2004) 385-392, doi:10.1002/sia.2010.

[99] M.H.W. Raes, M. Smeets, A novel measuring method of testing ITO protective coatings for use in mobile phone LCDs, in: International Symposium on Advanced Packaging Materials: Processes, Properties and Interfaces, IEEE, 2005, pp. 144-148.

[100] K.A. Sierros, N.J. Morris, K. Ramji, D.R. Cairns, Stress-corrosion cracking of indium tin oxide coated PET for flexible optoelectronic devices, Thin Solid Films 517 (2009) 2590-2595.

[101] M. Goto, A. Kasahara, Y. Konishi, T. Oishi, M. Tosa, K. Yoshihara. "Frictional property of zinc oxide coating films observed by lateral force microscopy" Jpn. J. Appl. Phys. 42 (2003) 4834-4836

[102] M. Goto, A. Kasahara, M. Tosa. "Reduction in frictional force of $\mathrm{ZnO}$ coatings in a vacuum" Jpn. J. Appl. Phys. 47 (2008) 8914-8916

[103] B. N. J. Persson, M. Sivebaek, V. N. Samoilov, Ke Zhao, A. I. Volokitin, Z. Zhang. "On the origin of Amonton's friction law" J. Phys: Condens. Matter 20 (2008) 395006-395017

[104] T. Xu, G. Wu, G. Zhang, Y. Hao. "The compatibility of $\mathrm{ZnO}$ piezoelectric film with micromachining process" Sensor Actuat. A 104 (2003) 61-67

[105] F. Fan, L. Lin, G. Zhu, W. Wu, R. Zhang, and Z. L. Wang. "Transparent Triboelectric Nanogenerators and Self-Powered Pressure Sensors Based on Micropatterned Plastic Films" Nano Letters. 12 (6) (2012) 3109-3114

[106] E. Fortunato, P. Nunes, A. Marques, D. Costa, H. Aguas, I. Ferreira, et al., Adv Eng Mater 4 (2002) 610.

[107] J.L. Ni, X.F. Zhu, Z.L. Pei, J. Gong, C. Sun, G.P. Zhang, J Phys D Appl Phys 42 (2009) 175404.

[108] S.Y. Chang, Y.C. Hsiao, Y.C. Huang, Surf Coat Technol 202 (2008) 5416.

[109] H. D. Espinosa, R.A. Bernal, M.Minary-Jolandan, "A Review of Mechanical and Electromechanical Properties of Piezoelectric Nanowires" Advanced Materials. 24 (2012) 4656-4675 
[110] R. Agrawal, B. Peng, E. E. Gdoutos, H. D. Espinosa, "Elasticity size effects of ZnO nanowires- a combined experimental-computational approach. Nano Letters. 8(11) (2008) 3668-3674

[111] F. Xu, Q. Qin, A. Mishra, Y. Gu, Y. Zhu, "Mechanical properties of ZnO nanowires under different loading modes" Nano Research 3 (2010) 271-280

[112] M. He, Y. Shi, W. Zhou, J.W. Chen, Y.J. Yan, J. Zhu "Diameter dependence of modulus in zinc oxide nanowires and the effect of loading mode: In situ experiments and universal core-shell approach" Applied Physics Letters 95 (2009) 091912

[113] B. Wen, J. E. Sader, J. J. Boland, "Mechanical Properties of ZnO Nanowires" Physical Review Letters 101 (2008), 175502

[114] M. Pugno, R. S. Ruoff, "Quantized fracture mechanics” Philosophical Magazine 84 (2004) 2829-2845

[115] N. M. Pugno, R. S. Ruoff, "Nanoscale Weibull statistics" Journal of Applied Physics 99 (2006) 024301-4

[116] M. H. Zhao, Z.L. Wang, S. X.Mao, "Piezoelectric characterization on individual zinc oxide nanobelt under piezoresponse force microscope", Nano Letters, 4 (2004) 587-590

[117] Z.L. Wang and J.H. Song "Piezoelectric Nanogenerators Based on Zinc Oxide Nanowire Arrays", Science, 14 April 2006: 242-246

[118] L-W Ji, S-M Peng, J-S Wu, W-S Shih, C-Z Wu, I-T Tang, "Effect of seed layer on the growth of well-aligned $\mathrm{ZnO}$ nanowires" Journal of Physics and Chemistry of Solids 70 (2009) 1359-1362

[119] S.Y. Chung, S. Kim, J-H Lee, K. Kim, S-W Kim, C-Y Kang, S-J Yoon, Y.S. Kim, "All-solutiong-processed flexible thin film piezoelectric nanogenerator" Adv. Mater. 24 (2012) 6022-6027

[120] B. Kumar, S-W Kim. "Recent advances in power generation through piezoelectric nanogenerators" J. Mater. Chem., 2011, 21, 18946

[121] Z.L. Wang, G. Zhu, Ya Yang, S. Wang, C. Pan, "Progress in nanogenerators for portable elecronics" Materialstoday 15(12) (2012)

[122] J.N. Patel, B. Kaminska, B.L. Gray, B.D. Gates, "A sacrificial SU-8 mask for direct metallization on PDMS” J. Micromech. Microeng. 19 (2009) 115014

[123] L. Lin, Y. Hu, C. Xu, Y. Zhang, R. Zhang, X. Wen, Z.L. Wang, "Transparent flexible nanogenerator as self-powered sensor for transportation monitoring" Nano Energy 2 (2013) 75-81

[124] J.A. Knapp, D.M. Follstaedt, S.M. Myers, J.C. Barbour, T.A. Friedmann, J. Appl. Phys. 85 (1999) 1460.

[125] H. Chai, B.R. Lawn, J. Mater. Res. 19 (2004) 1752.

[126] R. Andersson, G. Toth, L. Gan, M.V. Swain, Eng. Fract. Mech. 61 (1998) 93.

[127] A.A. Abdallah, P.C.P. Bouten, J.M.J. den Toonder, G. de With, Surf. Coat. Technol. 205 (2011) 3103.

[128] V.N. Zhitomirsky, T. David, R.L. Boxman, S. Goldsmith, A. Verdyan, Ya.M. Soifer, L. Rapoport, Thin Solid Films 492 (2005) 187.

[129] C.W. Chien, C.H. Wu, Y.T. Tsai, Y.C. Kung, C.Y. Lin, P.C. Hsu, H.H. Hsieh, C.C. Wu, Y.H. Yeh, C.M. Leu, T.M. Lee, IEEE Trans. Electron Devices 58 (2011) 1440.

[130] Y. Sun, W.M. Choi, H. Jiang, Y.Y. Huang, J.A. Rogers, Nat. Nanotechnology. 1 (2006) 201. 
[131] S.I. Park, J.H. Ahn, X. Feng, S. Wang, Y. Huang, J.A. Rogers, Adv. Funct. Mater. 18 (2008) 2673.

[132] S.J. Bull, Surf. Coat. Tech. 50 (1991) 25.

[133] K.A. Sierros, D.R. Cairns, J.S. Abell, S.N. Kukureka, Thin Solid Films 518 (2010) 2623.

[134] K.L. Johnson, Contact mechanics, Cambridge University Press, Cambridge, pp. 90-95 1987 (Chapter 4).

[135] G. Kourtesis, G.M. Renwick, A.C. Fisher-Cripps, M.V. Swain, J. Mater. Sci. 32 (1997) 4493.

[136] Y. Rao, J. Greener, C.A. Avila-Orta, B.S. Hsiao, T.N. Blanton, Polymer 49 (2008) 2507.

[137] Y. Leterrier, J. Andersons, Y. Pitton, J.A.E. Manson, J. Polym. Sci. Pol. Phys. 35

[138] P.C.P. Bouten, P.J. Slikkerveer, Y. Leterrier, in: G.P. Crawford (Ed.), Flexible Flat Panel Displays, John Wiley \& Sons, 2005, p. 99.

[139] H.D. Espinosa, B.C. Prorok, J. Mater. Sci. 38 (2003) 4125.

[140] H. Aziz, G. Xu, Synth. Met. 80 (1996) 7.

[141] J.H. Shin, S.H. Shin, J.I. Park, H.H. Kim, Properties of dc magnetron sputtered indium tin oxide films on polymeric substrates at room temperature, Journal of Applied Physics 89 (2001) 5199-5203.

[142] M. Scholten, J.E.A.M. van den Meerakker, J. Electrochem. Soc. 140 (1993) 471.

[143] J.E.A.M. van den Meerakker, P.C. Baarslag, W. Welrave, T.J. Vink, J.L.C. Daams, Thin Solid Films 266 (1995) 152.

[144] W.-F.Wu, B.-S.Chiou, "Mechanical properties of r.f. magnetron sputtered indium tin oxide films", Thin Solid Films 293 (1997) 244-250.

[145] D.R. Cairns, D.C. Paine, G.P. Crawford, The mechanical reliability of sputtercoated indium tin oxide polyester substrates for flexible display and touchscreen applications, in: 44th Annual Technical Conference Proceedings, Philadelphia, Society of Vacuum Coaters, 2001, pp. 160-165.

[146] Y.N. Kim, M.S. Jeon, M.C. Shin, S.M. Lee, H.S. Lee, Adhesive and wear properties of indium tin oxide (ITO) thin films deposited by RF magnetron sputter, Key Engineering Materials 317-318 (2006) 381-384.

[147] W. Bijnens, I. De Wolf, J. Manca, J.D. Haen, T.D. Wu, M.D 'Olieslaeger, E. Beyne, R. Kiebooms, D. Vanderzande, J. Gelan, W. De Ceuninck, L. De Schepper, L.M. Stals, Synth. Met. 96 (1998) 87.

[148] I. Kortidis, K. Moschovis, F.A. Mahmoud, G. Kiriakidis. "Structural analysis of aerosol spray pyrolysis $\mathrm{ZnO}$ films exhibiting ultra low ozone detection limits at room temperature" Thin Solid Films 518 (2009) 1208-1213

[149] G. Kiriakidis, M. Suchea, S. Christoulakis, P. Horvath, T. Kitsopoulos, J. Stoemenos, Thin Solid Films 515 (2007) 8577. 